\title{
Wasserhaushalt und Wassernutzungseffizienz von vier perennierenden Pflanzenarten im Vorland einer zentralasiatischen Flussoase
}

\author{
Dissertation \\ zur Erlangung des Doktorgrades \\ der Mathematisch-Naturwissenschaftlichen Fakultät \\ der Georg-August-Universität zu Göttingen
}

\author{
vorgelegt von \\ Andrea Foetzki \\ aus Kassel
}

Göttingen, 16.12.2002 
D 7

Referent: Prof. Dr. M. Runge

Korreferent: Prof. Dr. C. Leuschner

Tag der mündlichen Prüfung: 30.01.2003

Diese Arbeit erscheint in den Berichten des Forschungszentrums Waldökosysteme

Reihe A, Band 186, ISSN 0939-1347. 
Wüsten sind in der Regel breitsäumige, ebene Landstriche, bei denen es infolge Wassermangels mit der Vegetation gesamtflächig hapert, außer an Stellen, wo unterirdische Quellen der Oasenentstehung Vorschub leisten.

Prof. Dr. Abdul Nachtigaller (in MOERS 1999) 



\section{Inhaltsverzeichnis}

Inhaltsverzeichnis

I

Liste der verwendeten Abkürzungen...............................................................II

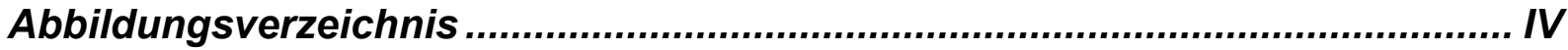

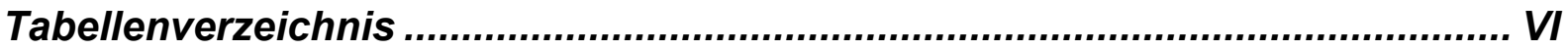

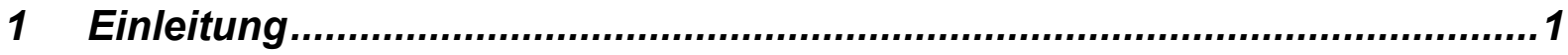

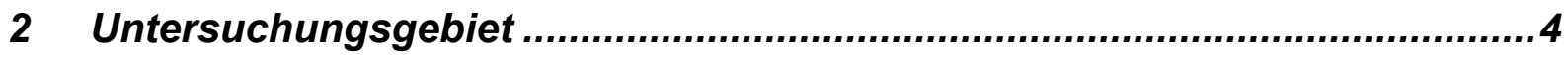

2.1 Geographie und Geologie ...................................................... 4

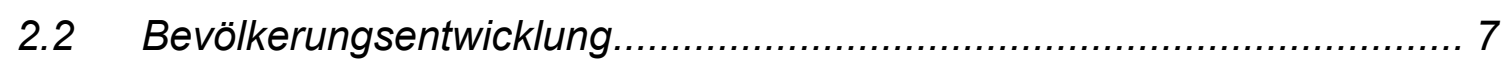

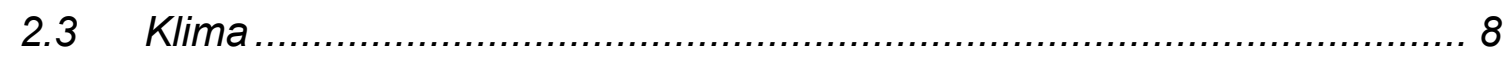

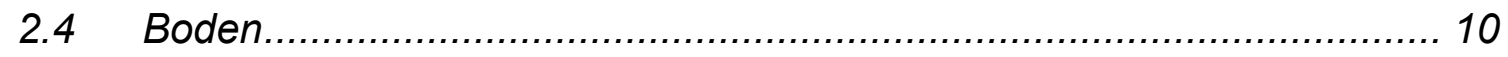

2.5 Pflanzenarten ....................................................................... 11

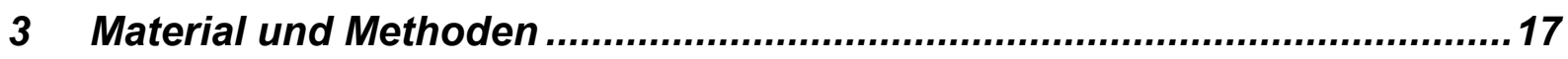

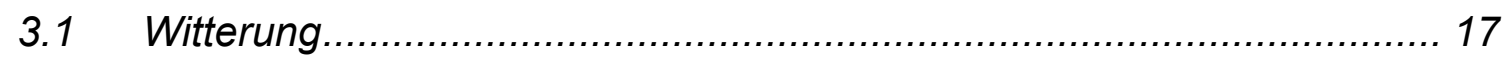

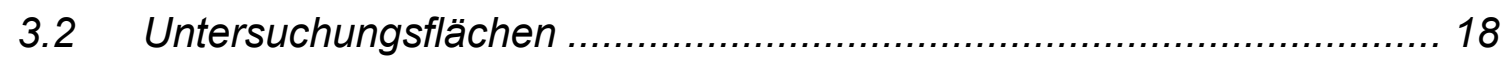

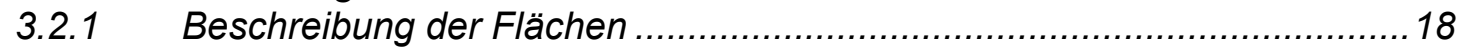

3.2.2 Experimentelle Behandlung .......................................................... 19

3.3 Bodenwassergehalt............................................................... 23

3.4 Entwicklung der Blattfläche und des Blattflächenindexes (LAI)............. 24

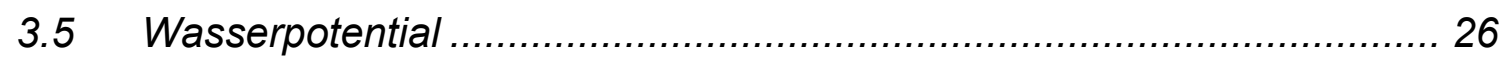

3.6 Transpiration und stomatäre Leitfähigkeit .................................. 27

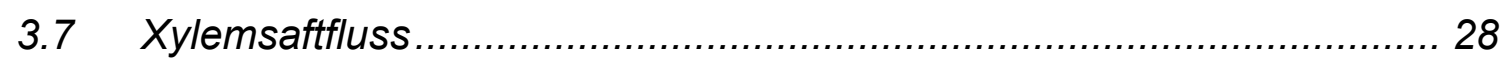

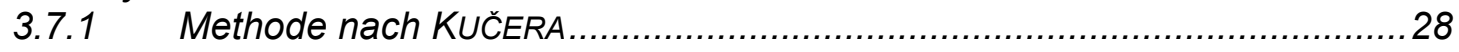

3.7.2 Methode nach GRANIER ................................................................. 31

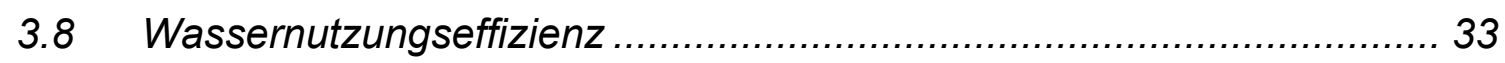

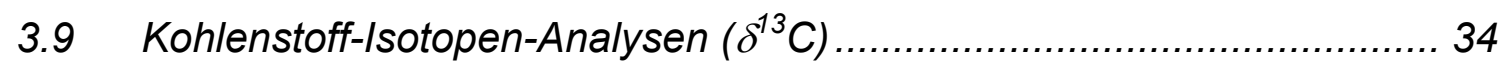

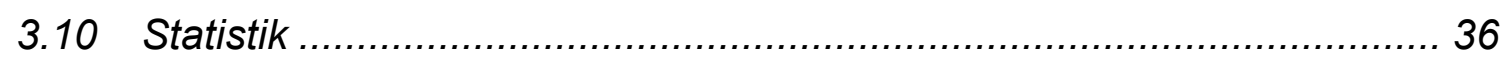

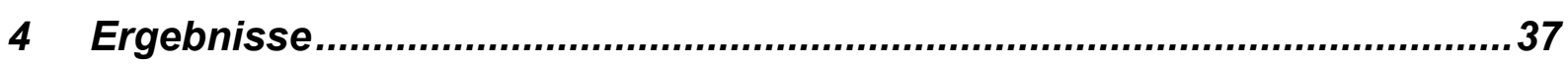

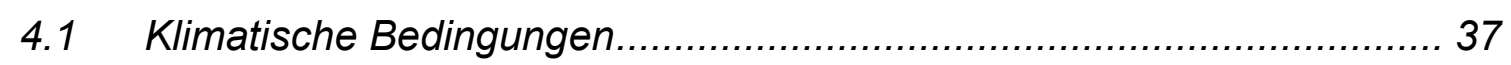

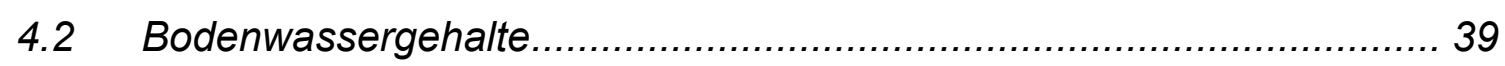

4.3 Entwicklung von Blattbiomasse und Blattflächenindex (LAI) .................43 43

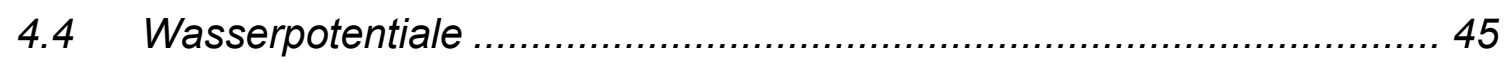

4.5 Transpiration und stomatäre Leitfähigkeit ..................................... 54 


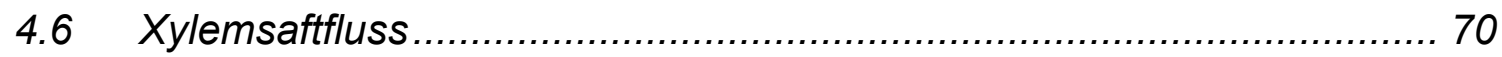

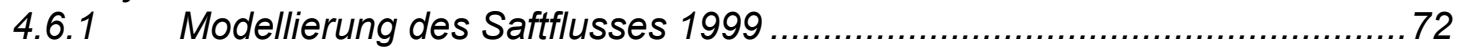

4.6.2 Saftflussmessungen und Modellierung 2000 ….................................. 79

4.6.3 Vergleich des aus Saftfluss- und Transpirationsmessungen ermittelten

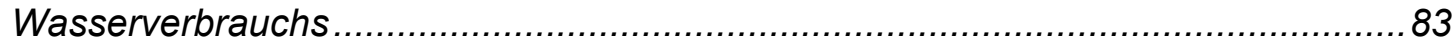

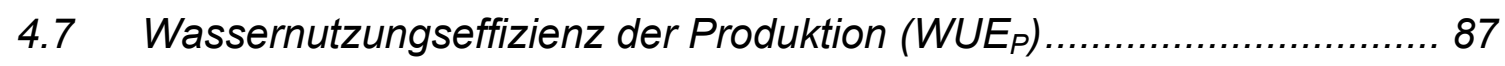

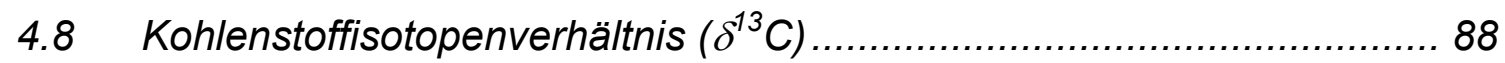

5 Diskussion

5.1 Die Oase Qira als Modell für die Desertifikations-Problematik am

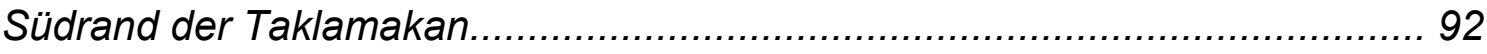

5.2 Wasserverfügbarkeit für Pflanzen im Oasenvorland von Qira............... 93

Bedingungen ohne Bewässerung ............................................................... 93

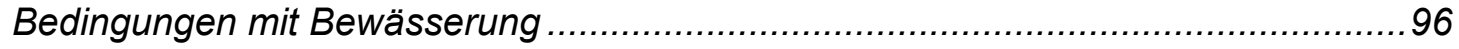

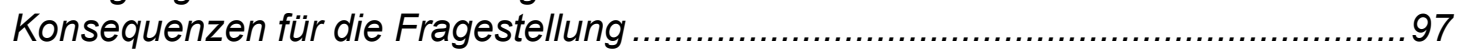

5.3 Wasserhaushalt der Pflanzen ........................................................ 98

5.3.1 Höhe und zeitlicher Verlauf der Wasserpotentiale........................................ 98

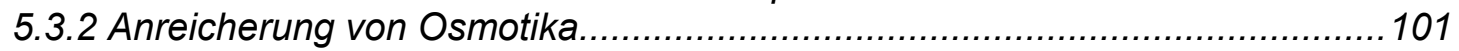

5.3.3 Transpiration und stomatäre Leitfähigkeit..................................................103

5.3.4 Effekte der experimentellen Bewässerung auf den Wasserhaushalt der

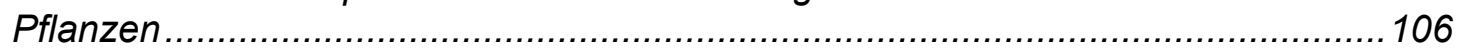

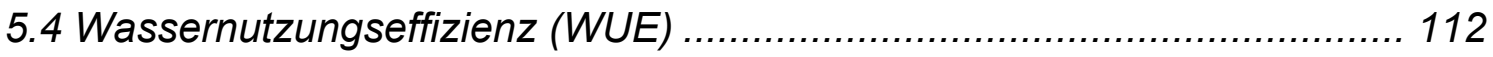

5.4.1 Wasserverbrauch der Arten berechnet aus Saftflussmessungen....................112

5.4.2 Vergleich von Transpiration und Saftfluss ...............................................113

5.4.3 Produktion der untersuchten Arten ....................................................... 117

5.4.4 Wassernutzungseffizienz der Produktion (WUEp)...................................... 118

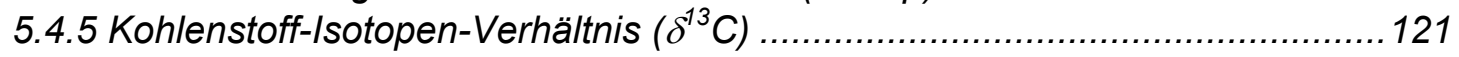

5.5 Nutzung der untersuchten Arten ................................................... 125

5.5.1 Auswirkung der experimentellen Nutzung auf den Wasserhaushalt...............126

5.5.2 Auswirkungen der experimentellen Nutzung auf die Produktion ....................127

5.5.3 Nutzung durch die Bevölkerung .......................................................... 128

5.5.4 Bedeutung der Wassernutzungseffizienz der genutzten Arten ...................... 131

5.6 Regeneration ..................................................................... 131

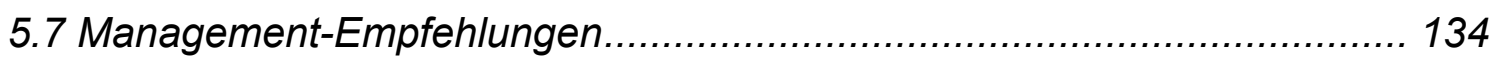

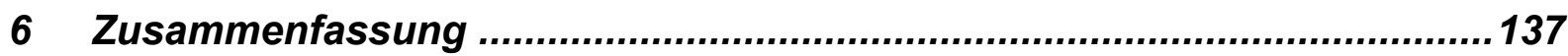

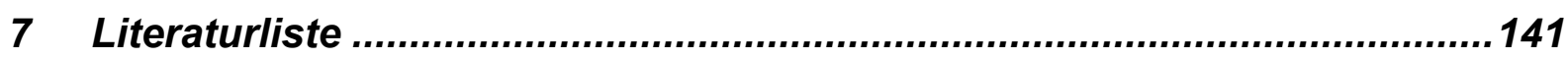

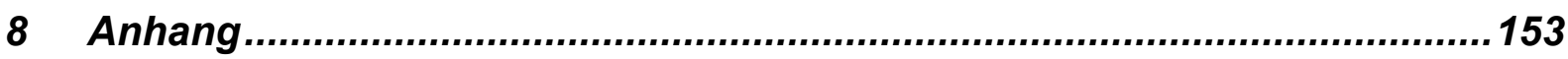




\section{Liste der verwendeten Abkürzungen}

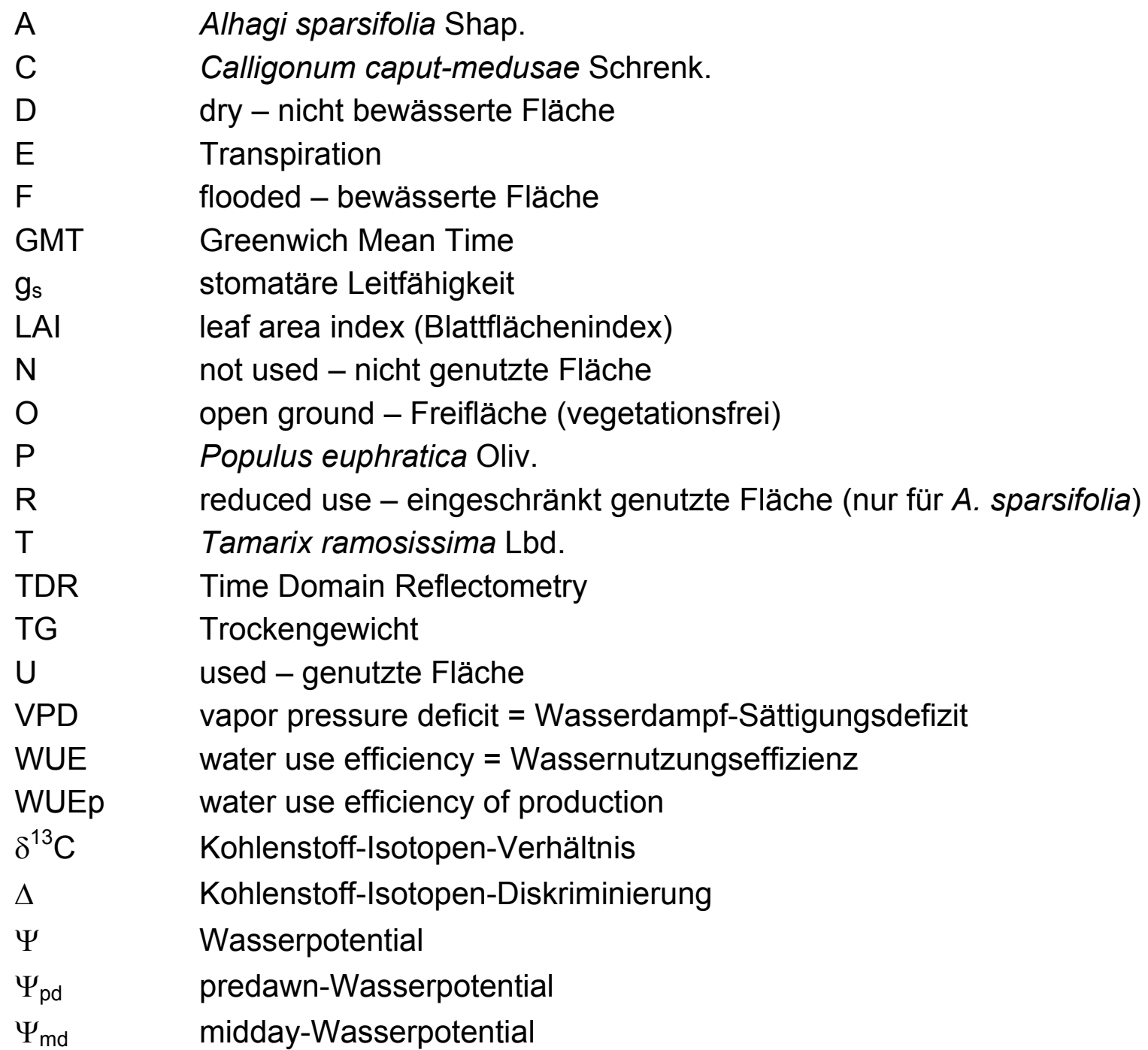




\section{Abbildungsverzeichnis}

Abb. 2.1.1: $\quad$ Lage des Untersuchungsgebietes in Nordwest-China.................. 4

Abb. 2.1.2: $\quad$ Lage der Untersuchungsflächen im Vorland der Oase Qira. ......... 6

Abb. 2.2.1: $\quad$ Bevölkerungsentwicklung der Präfektur Hotan von 1911-2000 und der Oase Qira von 1965-1998............................................. 7

Abb. 2.3.1: $\quad$ Klimadiagramm der Oase Qira nach WALTER (1990).................. 9

Abb. 2.3.2: $\quad$ Monatsmittel der Lufttemperatur 1993-2000............................... 10

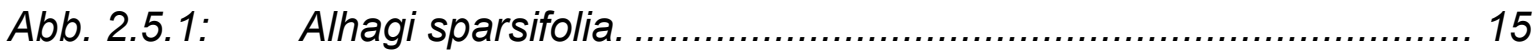

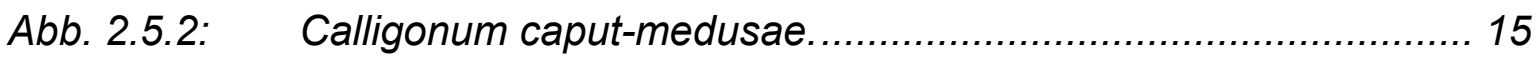

Abb. 2.5.3: $\quad$ Populus euphratica............................................................. 16

Abb. 2.5.4: $\quad$ Tamarix ramosissima. ........................................................... 16

Abb. 3.7.1: $\quad$ Tagesgänge des Saftflusses von Alhagi sparsifolia mit Mittagslücken. ................................................................... 30

Abb. 4.1.1: $\quad$ Monatliche Strahlungssummen der Globalstrahlung und Monatsmitteltemperaturen 1999 und 2000.................................. 38

Abb. 4.1.2: $\quad$ Tagesmittelwerte des Wassersättigungsdefizits (VPD) während der Vegetationsperioden der Untersuchungsjahre 1999 und 2000.

Abb. 4.2.1: Bodenwassergehalte (Vol. \%) auf den Untersuchungsflächen 1999.

Abb. 4.2.2: Bodenwassergehalte (Vol. \%) auf den Untersuchungsflächen 2000

Abb. 4.3.1: $\quad$ Entwicklung der Blattbiomasse bzw. des Blattflächenindexes (LAI) der untersuchten Arten in der Vegetationsperiode 1999 und 2000.

Abb. 4.4.1: Tagesgänge des Blattwasserpotentials und des Wasserdampfsättigungsdefizits (VPD) von Alhagi sparsifolia 1999.

Abb. 4.4.2: $\quad$ Tagesgänge des Blattwasserpotentials und des Wasserdampfsättigungsdefizits (VPD) von Calligonum caput-medusae 1999..... 47

Abb. 4.4.3: $\quad$ Tagesgänge des Blattwasserpotentials und des Wasserdampfsättigungsdefizits (VPD) von Tamarix ramosissima 1999. 48

Abb. 4.4.4: $\quad$ Tagesgänge des Blattwasserpotentials und des Wasserdampfsättigungsdefizits (VPD) von Populus euphratica 1999. 49

Abb. 4.4.5: Predawn-Wasserpotentiale der untersuchten Arten 1999............... 50

Abb. 4.4.6: Predawn- und midday-Wasserpotentiale von Alhagi sparsifolia und Calligonum caput-medusae 2000.

Abb. 4.4.7: Predawn- und midday-Wasserpotentiale von Tamarix ramosissima und Populus euphratica 2000.

Abb. 4.5.1: Transpiration von Alhagi sparsifolia 1999................................ 55

Abb. 4.5.2: Stomatäre Leitfähigkeit von Alhagi sparsifolia 1999...................... 56

Abb. 4.5.3: Transpiration von Alhagi sparsifolia 2000. 
Abb. 4.5.4: Stomatäre Leitfähigkeit von Alhagi sparsifolia 2000..................... 58

Abb. 4.5.5: $\quad$ Transpiration von Calligonum caput-medusae 1999................... 59

Abb. 4.5.6: $\quad$ Stomatäre Leitfähigkeit von Calligonum caput-medusae 1999.... 60

Abb. 4.5.7: Transpiration von Calligonum caput-medusae $2000 \ldots \ldots \ldots \ldots \ldots \ldots . . . . . .61$

Abb. 4.5.8: $\quad$ Stomatäre Leitfähigkeit von Calligonum caput-medusae 2000... 62

Abb. 4.5.9: $\quad$ Transpiration von Tamarix ramosissima 1999...........................63 63

Abb. 4.5.10: $\quad$ Stomatäre Leitfähigkeit von Tamarix ramosissima 1999............ 64

Abb. 4.5.11: Transpiration von Tamarix ramosissima 2000.......................... 65

Abb. 4.5.12: $\quad$ Stomatäre Leitfähigkeit von Tamarix ramosissima 2000............ 66

Abb. 4.5.13: Transpiration von Populus euphratica 1999..............................67 67

Abb. 4.5.14: $\quad$ Stomatäre Leitfähigkeit von Populus euphratica 1999. ............... 68

Abb. 4.5.15: Transpiration von Populus euphratica 2000............................ 69

Abb. 4.5.16: Stomatäre Leitfähigkeit von Populus euphratica 2000. ............... 69

Abb. 4.6.1: Verhältnisse der Saftflussraten der Sensoren 2-5 vs. Sensor 1 am Beispiel von Alhagi sparsifolia............................................... 73

Abb. 4.6.2: Globalstrahlung vs. Saftflussrate des Sensors 1 von Alhagi sparsifolia im Juli 1999.

Abb. 4.6.3: Gemessene und modellierte Tagessummen des Saftflusses von Alhagi sparsifolia 1999........................................................ 75

Abb. 4.6.4: Modellierte Tagessummen des Saftflusses von Alhagi sparsifolia, Calligonum caput-medusae und Tamarix ramosissima 1999........ 77

Abb. 4.6.5: Modellierte Tagessummen des Saftflusses von Populus euphratica 1999.

Abb. 4.6.6: Modellierte Tagessummen des Saftflusses von Alhagi sparsifolia, Calligonum caput-medusae und Tamarix ramosissima 2000. ....... 80

Abb. 4.6.7: Tamarix ramosissima: Saftflussrate und Witterungsbedingungen vom

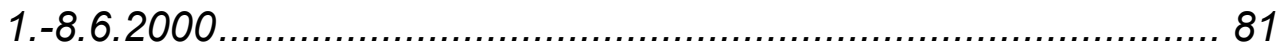

Abb. 4.6.8: Saftlusstagessummen von Populus euphratica 2000................... 82

Abb. 4.6.9: Vergleich des Wasserverbrauchs gemessen durch Saftfluss und Transpiration der untersuchten Arten 1999. 84

Abb. 4.6.10: Vergleich des Wasserverbrauchs gemessen durch Saftfluss und Transpiration der untersuchten Arten 2000.

Abb. 4.6.11: Methodenvergleich der Messung des Tageswasserverbrauchs gemessen mit dem Saftflusssystem nach KUČERA und mit dem LI-1600.

Abb. 4.7.1: Wassernutzungseffizienz der Produktion (WUEp) von A. sparsifolia, C. caput-medusae und T. ramosissima 1999. 87

Abb. 4.8.1: Kohlenstoff-Isotopenverhältnisse $\left(\delta^{13} C\right)$ der vier untersuchten Arten im Jahresverlauf 1999. 89

Abb. 4.8.2: Kohlenstoff-Isotopenverhältnisse $\left(\delta^{13} \mathrm{C}\right)$ von A. sparsifolia im Jahr 2000. 


\section{Tabellenverzeichnis}

Tab. 1: Größe, Lage und Behandlungsvarianten der Untersuchungsflächen....... 21

Tab. 2: Bestandesdichte und Begleitarten auf den Untersuchungsflächen 2000. 22

Tab. 3: Messtiefe und Typ der TDR-Sonden auf den Untersuchungsflächen. ..... 23

Tab. 4: Maximale Differenz im Wasserpotential 1999. .................................... 51

Tab. 5: Saftflusssummen von Calligonum caput-medusae und Witterungs-

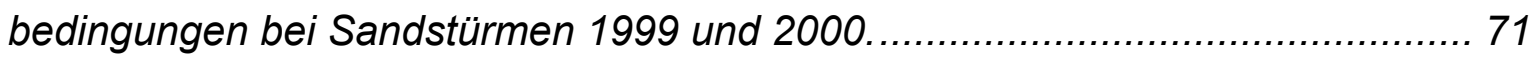

Tab. 6: Wasserverbrauch der untersuchten Arten 1999.................................116

Tab. 7: Wassernutzungseffizienz der Produktion (WUEp) 1999.......................120

Tab. 8: Monatsmittel der Lufttemperaturen der Jahre 1999 und 2000................123 


\section{Einleitung}

Das Phänomen der Landdegradation tritt in allen ariden und semi-ariden Gebieten weltweit auf (UNEP 1992). Es bezeichnet den Verlust wesentlicher Eigenschaften eines Ökosystems oder eine Abnahme des Potentials, die vitalen ökonomischen und ökologischen Funktionen zu erfüllen. Für die Degradation in Trockengebieten wurde der Begriff Desertifikation geprägt. Desertifikation wurde im Laufe der Zeit unterschiedlich definiert (z.B. RAPP 1974, DREGNE 1978, MeNSCHING 1990, Thomas \& MiddLeton 1994, Le Houérou 1996, KATYAL \& VLeK 2000): gemeinsam ist allen Definitionen, dass sie Desertifikation hauptsächlich als die anthropogen bedingte Landdegradation ansehen, die durch die Übernutzung natürlicher Ressourcen verursacht wird und $\mathrm{zu}$ einer kontinuierlichen Abnahme der wirtschaftlichen Produktivität führt. Durch die UNCED 1992 wurde eine klare Definition eingeführt, die Desertifikation bezeichnet als „Land degradation in arid, semiarid and dry sub-humid areas, resulting from various factors including climatic variations and human activities".

Im besiedelten Randbereich der zentralasiatischen Wüsten wird Desertifikation durch ständig steigenden Wasserbedarf der bewässerungsintensiven Landwirtschaft, Ausweitung der Landwirtschaft auf ungeeignete Flächen und die Übernutzung der indigenen Vegetation hervorgerufen (FAN 1995, WU \& CI 2002). Ein Grund für die Zerstörung des unverzichtbaren, Sand fixierenden Vegetationsgürtels im Übergangsbereich zwischen Oase und Wüste ist der stark zunehmende Bedarf der rasch wachsenden Bevölkerung an Viehfutter und Brennstoff (ZHU \& LIU 1988). Die daraus resultierende Übersandung von landwirtschaftlich genutzter Fläche ist eine ständige Bedrohung für die Oasen. Als ein Beispiel für diese Problematik kann man die Oase Qira am Südrand der Taklamakan in der Autonomen Uighurischen Region Xinjiang, Nordwestchina, heranziehen, in der das hier vorgestellte Projekt durchgeführt wurde.

Ziel des Projekts „Ecological basis for a sustainable management of the indigenous vegetation in a Central Asian desert" (EU Projekt ERBIC18-CT980275), in dessen Rahmen die vorliegende Arbeit durchgeführt wurde, ist es, die 
ökologischen Grundlagen (Wasserhaushalt, Produktivität, Vegetationszusammensetzung) für ein Managementkonzept zusammenzutragen, das die Nachhaltigkeit der Nutzung der Vegetation garantiert, so dass deren schützender Effekt gegen Sanddrift erhalten bleibt.

Wasser ist die am stärksten limitierende Ressource für die pflanzliche Produktivität in ariden Ökosystemen. Die Wasserverfügbarkeit, einer der wichtigsten Umweltfaktoren, die das Wachstum/das Leben der Pflanzen beeinflussen, wird schon seit langem in Wüsten untersucht (z.B. CALDWELL 1985, Comstock und EHLERINGER 1992, Donovan und EhleRINGer 1992). Die jahreszeitliche und interannuelle Variabilität der Wasserverfügbarkeit in ariden und semi-ariden Gebieten führt zu Anpassungsmechanismen, durch die die Pflanzen den Wasserverbrauch einschränken bzw. die Wassernutzungseffizienz erhöhen.

Es stehen Untersuchungen zum Wasserhaushalt auf Blatt- und Bestandesebene sowie zur Wassernutzungseffizienz der Produktion der dominierenden Pflanzenarten des Untersuchungsgebietes im Mittelpunkt der vorliegenden Arbeit.

Es wurde vermutet, dass die Vegetation im Oasenvorland von unregelmäßig alle zwei bis drei Jahre auftretenden Überflutungen abhängig ist und die untersuchten Arten Anzeichen von Trockenstress aufweisen, je länger die letzte Überflutung zurückliegt. Daher wurde experimentell der Einfluss einer einmaligen Bewässerung auf den Wasserhaushalt (Transpiration, stomatäre Leitfähigkeit, Wasserpotential, Xylemsaftfluss) etablierter Bestände der vier Arten Alhagi sparsifolia Shap., Calligonum caput-medusae Schrenk., Tamarix ramosissima Lebed. und Populus euphratica Oliv. untersucht.

Da die untersuchten Pflanzenarten als Brennholz, Baumaterial und Viehfutter genutzt werden, wurde in einem zusätzlichen Experiment diese Nutzung durch die Bevölkerung simuliert und wiederum die Auswirkung auf den Wasserhaushalt betrachtet.

Die Wassernutzungseffizienz der Produktion (WUEp) wurde für die untersuchten Arten aus der verbrauchten Wassermenge und dem Biomassezuwachs berechnet. Um eine Langzeitschätzung der Wassernutzungseffizienz der Pflanzen zu erhalten, kann das Kohlenstoffisotopenverhältnis $\left(\delta^{13} \mathrm{C}\right)$ ihrer Biomasse herangezogen werden. $\delta^{13} \mathrm{C}$ ist über den Mechanismus der Stomataregulation mit der 
Physiologie verknüpft und wurde daher als Indikator für den Wasserzustand der Pflanze (FARQUHAR et al. 1982) und die Wassernutzungseffizienz (WUE) vorgeschlagen (FARQUHAR et al. 1989). Die Wassernutzungseffizienz der Produktion kann bei der Auswahl geeigneter Pflanzen für Regenerationsmaßnahmen behilflich sein.

Die Ergebnisse dieser Untersuchung können eine Grundlage zum Abschätzen der erforderlichen Wassermenge und zum Berechnen des Verhältnisses zwischen Wasserinvestition und ökonomischem Nutzen für verschiedene ManagementAlternativen bieten. Es wird erwartet, dass die Ergebnisse allgemeine Gültigkeit für zentralasiatische Wüsten haben und einen Beitrag dazu leisten können, die Degradation der Vegetation in diesen Gebieten aufzuhalten. 


\section{Untersuchungsgebiet}

\subsection{Geographie und Geologie}

Die Taklamakan liegt in der Autonomen Uighurischen Provinz Xinjiang im Nordwesten Chinas und ist mit rund $337000 \mathrm{~km}^{2}$ die größte Wüste Chinas. Sie nimmt einen Großteil des Tarimbeckens ein, eines tektonisch angelegten abflusslosen Hochbeckens, das von 1400 m über NN im Westen bis 800 m über NN im Osten abfällt (Abb. 2.1.1). Die extrem ariden Bedingungen (YANG 1991) werden durch die kontinentale Lage und die umschließenden Bergketten des Tian Shan im Norden, des Pamir und Karakorum im Westen und des Kunlun Shan und Altun Shan im Süden bedingt. Die Taklamakan besteht zu $85 \%$ aus mobilen Sanddünen (YANG 1991).

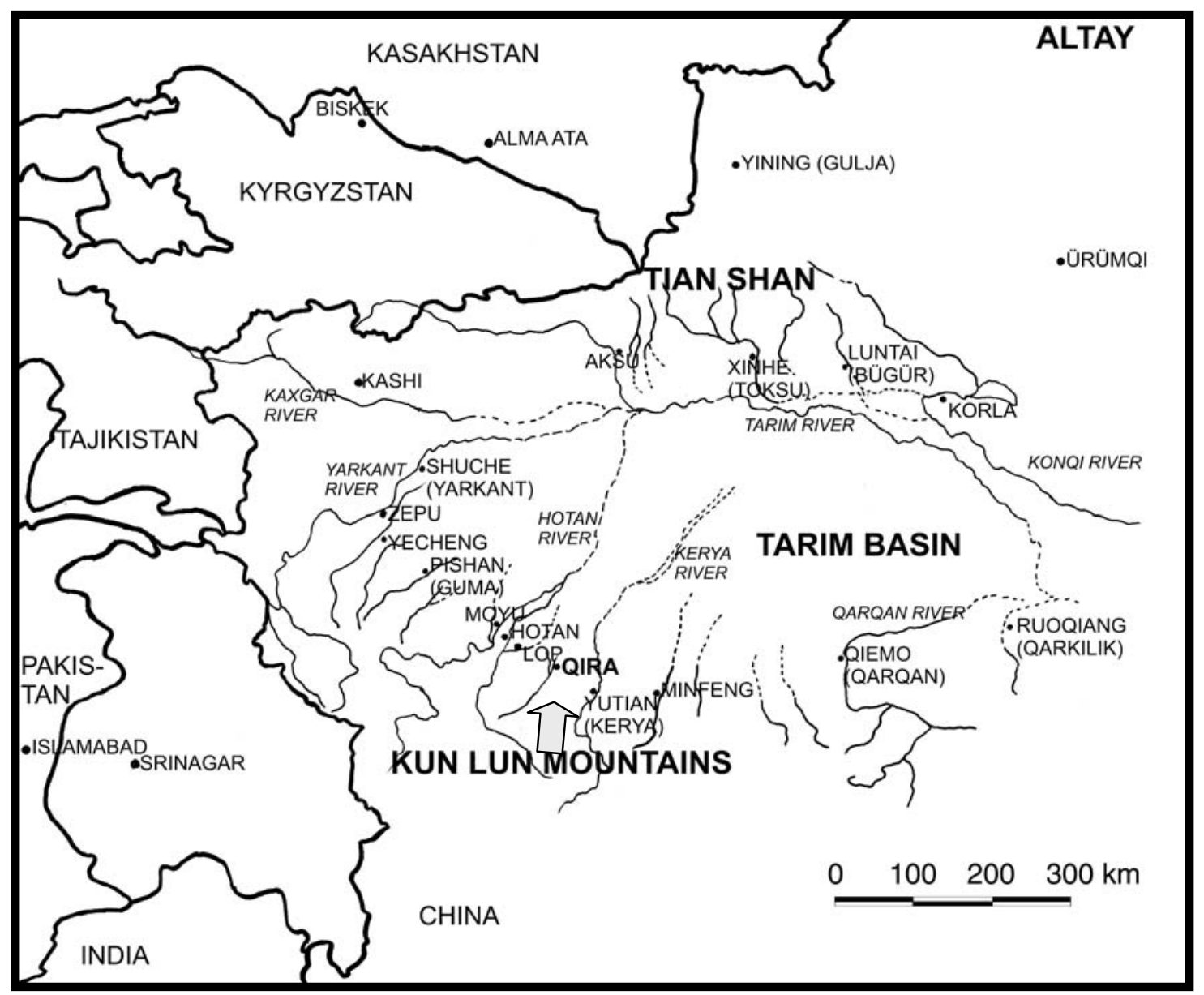

Abb. 2.1.1: Lage des Untersuchungsgebietes in Nordwest-China.

Das Tarimbecken ist mit den umgebenden Bergketten und den Oasen am Rand der Taklamakan dargestellt. Das Untersuchungsgebiet befindet sich am Rand der Oase Qira (chinesisch: Cele) im Bezirk Hotan. 
Das Untersuchungsgebiet befand sich am Südrand der Taklamakan in der Präfektur Hotan. Dieser Bezirk hat eine Fläche von $245000 \mathrm{~km}^{2}$, von der nur $3,75 \%$ von Oasen bedeckt sind, und teilt sich in sieben Verwaltungseinheiten. Qira County ist eine dieser Einheiten und besteht aus der Hauptoase Qira und mehreren kleinen Oasen am Fuß des Kunlun. Die Untersuchungsflächen wurden

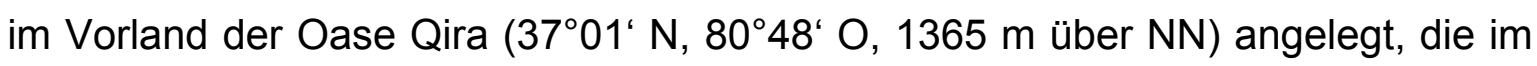
Übergangsbereich der sich von den Ausläufern des Kunlun erstreckenden glazifluvialen Gobischwemmfächern und der Wüste liegt (JÄKEL 1991, HöVERMANN 1990). Die von den vergletscherten Randgebirgen ins Tarimbecken abfließenden Flüsse versickern meist im Saumbereich der Wüste, manche enden in Salzseen oder -sümpfen der Taklamakan.

Am Südrand der Taklamakan treten in den Monaten Juni bis August infolge der Schneeschmelze im Gebirge Hochwasser auf, bei denen 75 \% der gesamten jährlichen Wassermenge abfließen. Die Monate mit dem niedrigsten Wasserstand sind März bis Mai; in diesem Zeitraum führt der Qira Fluss nur etwa $9 \%$ der Jahreswassermenge (XIA et al. 1993). Gleichzeitig ist der Wasserbedarf in der Landwirtschaft im Frühjahr sehr hoch. In dieser Jahreszeit muss größtenteils mit Grundwasser bewässert werden, das aus 70 - 90 m Tiefe gepumpt wird. Der Fluss wird in trockenen Jahren komplett über ein Kanalsystem durch die Oase geleitet und dort zur Bewässerung der Feldkulturen genutzt. Nur das Restwasser der Oasenwirtschaft fließt durch die offen ins Vorland mündenden Bewässerungskanäle zur Wüste hin ab. In niederschlagsreicheren Jahren fließt ein Teil des Flusses durch ein System aus alten und neuen Flussbetten kilometerweit in die Wüste hinein (siehe Abb. 2.1.2). 


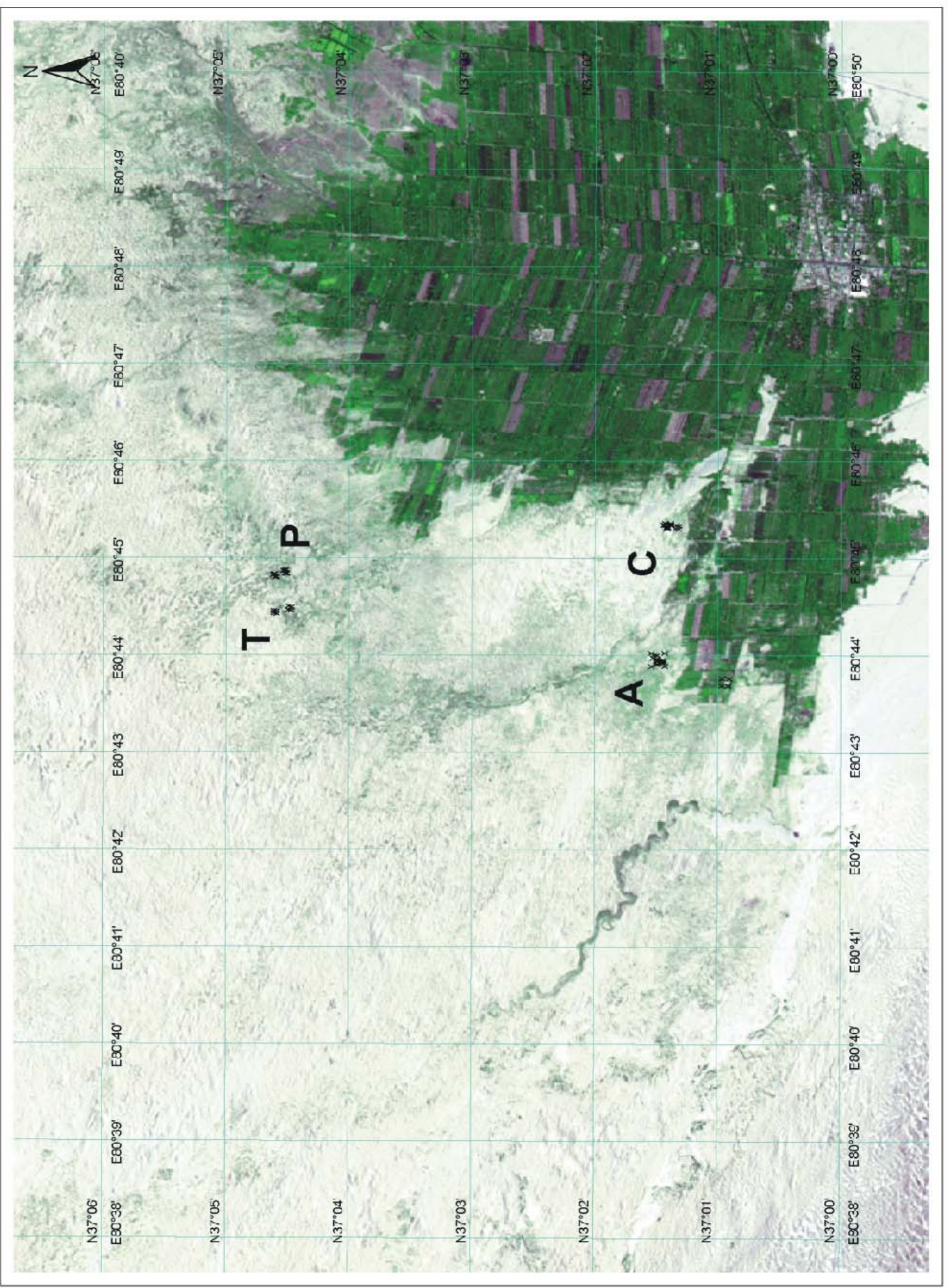

Abb. 2.1.2: Lage der Untersuchungsflächen im Vorland der Oase Qira.

Die Untersuchungsflächen befinden sich im westlichen Oasenvorland in einem Gürtel natürlicher Vegetation, der die landwirtschaftlich genutzte Fläche der Oase zur Wüste hin abgrenzt. Flächenkürzel: A Alhagi sparsifolia, C Calligonum caput-medusae, T Tamarix ramosissima, $\mathbf{P}$ Populus euphratica. 


\subsection{Bevölkerungsentwicklung}

Die Autonome Uighurische Region Xinjiang ist die größte chinesische Provinz und nimmt ein Sechstel der Landfläche Chinas ein. Ebenso wie im restlichen China ist ein starkes Bevölkerungswachstum zu verzeichnen. Seit 1949 stieg die Gesamtbevölkerung Xinjiangs von 4,33 auf 17,18 Millionen (1997). Xinjiang ist nach Yunnan die Provinz mit den meisten Volksgruppen in China, 20 der staatlich anerkannten 56 Nationalitäten Chinas leben hier.

In Xinjiang leben nur etwa 6,6 Millionen (38,4\%) Han, die im Rest Chinas die größte Bevölkerungsgruppe stellen und vor allem seit den 1950er Jahren in den Nordwesten zugewandert sind. Etwa 60 \% der Bevölkerung bestehen aus anderen Nationalitäten, von denen mit etwa 8 Millionen (46,7 \%) die Uighuren den größten Anteil ausmachen (MACKERRAS 2001). Die Bevölkerung der Oase Qira hat sich seit den 1960er Jahren fast verdreifacht (Abb. 2.2.1). Zum Vergleich ist der exponentielle Anstieg der Bevölkerung in der gesamten Präfektur Hotan dargestellt.

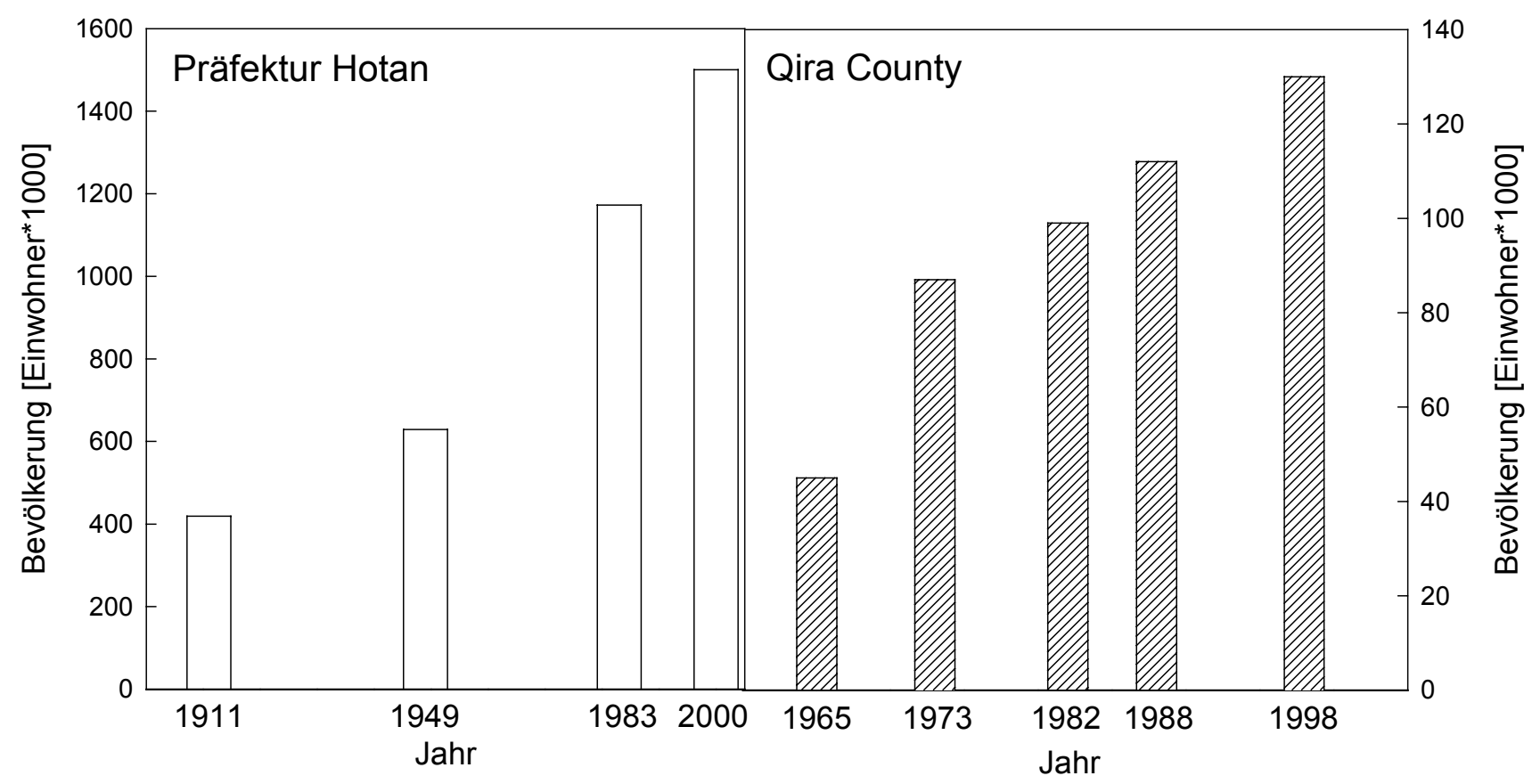

Abb. 2.2.1: Bevölkerungsentwicklung der Präfektur Hotan von 1911-2000 und der Oase Qira von 1965-1998.

Daten der lokalen Behörden. 
In Xinjiang waren seit langer Zeit Oasenfeldbau mit kleinflächigen Mischkulturen (bei den Uighuren) und halbnomadische Viehhaltung (z.B. bei den Kasachen und Tadschiken) verbreitet. Mit den starken Umsiedlungsschüben der 70er Jahre, die mit der Fertigstellung der Eisenbahnlinie von Lanzhou nach Urumqi einher gingen, erfolgten große Landerschließungsmaßnahmen. Erschließung wenig ertragreicher Flächen und nicht angepasste Bewässerung führten zu ökologischen Problemen, die noch heute ihre Auswirkungen zeigen (HAMANN \& HALIK 1998, MACKERRAS 2001).

\subsection{Klima}

Das Klima im Untersuchungsgebiet ist extrem kontinental und arid mit kalten trockenen Wintern und trockenheißen Sommern. Die jährliche mittlere Niederschlagssumme beträgt nur 33,2 mm (WALTER 1990) und die Niederschläge fallen sehr variabel. Die häufigsten Niederschlagsereignisse treten im Mai und Juni auf. Die mittlere Jahrestemperatur beträgt $11,9^{\circ} \mathrm{C}$ (Abb. 2.3.1).

Die sommerlichen Tageshöchsttemperaturen liegen meist zwischen 35 und $40{ }^{\circ} \mathrm{C}$ (Temperaturmaximum im Untersuchungszeitraum 1999 - 2000: 40,6 ${ }^{\circ} \mathrm{C}$ ), die Nächte sind mit einer mittleren Temperatur von $17,6^{\circ} \mathrm{C}$ vergleichsweise kühl. Im Winter können die Temperaturen auf etwa $-20{ }^{\circ} \mathrm{C}$ fallen (Temperaturminimum im Untersuchungszeitraum 1999 - 2000: $-16,4^{\circ} \mathrm{C}$ ), meistens liegen sie aber eher im Bereich von -10 bis $-15{ }^{\circ} \mathrm{C}$ nachts und -5 bis $0{ }^{\circ} \mathrm{C}$ tagsüber. (Abb. 2.3.2)

Die jährliche potentielle Evaporation beträgt etwa 2600 mm (XIA et al. 1993).

Die Vegetationsperiode beginnt Anfang April und endet im Oktober. Der letzte Frosttag im Frühjahr 2000 war der 26. März $\left(-5,1^{\circ} \mathrm{C}\right)$, im Herbst 1999 wurde der erste Frost am 15. Oktober gemessen (-0,4 $\left.{ }^{\circ} \mathrm{C}\right)$. Die Untersuchungsjahre 1999 und 2000 unterscheiden sich nicht sehr von den vorangegangenen Jahren, der Winter 1999/2000 war etwas milder (Abb. 2.3.2).

Die relative Luftfeuchte sinkt im Sommerhalbjahr nachmittags häufig auf unter $10 \%$. Nur in den frühen Morgenstunden oder nach kurzen Regenschauern treten Werte von über $50 \%$ auf (maximal $90 \%$ ). Es wurde kein Taufall beobachtet. 


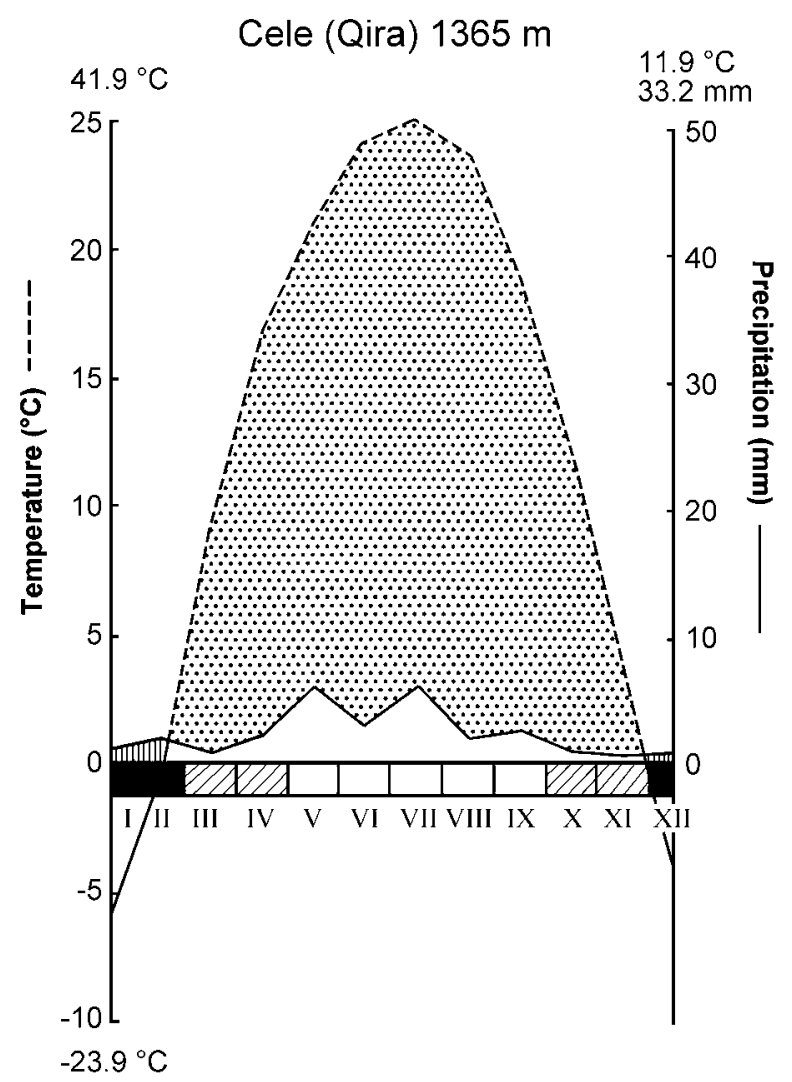

Abb. 2.3.1: Klimadiagramm der Oase Qira nach WALTER (1990).

Daten der staatlichen Wetterstation in Qira (nach ZHANG XIMING, unveröffentlicht). Die absoluten Maximum- und Minimumtemperaturen sind über bzw. unter der linken y-Achse angegeben, die Jahresmitteltemperatur und der Jahresniederschlag über der rechten y-Achse. Auf der x-Achse sind Monate mit einer mittleren Tagestemperatur unter $0{ }^{\circ} \mathrm{C}$ in schwarz, Monate mit einer absoluten Minimumtemperatur unter $0^{\circ} \mathrm{C}$ schraffiert dargestellt.

Im südwestlichen Teil des Tarimbeckens ist die Hauptwindrichtung Nordwest, im südöstlichen Tarimbecken dagegen Nordost. Qira liegt im Übergangsbereich dieser vorherrschenden Windsysteme (YANG 1991) mit leichter Präferenz der nordwestlichen Winde. Das Gebiet ist geprägt von häufigen Staubstürmen, die vor allem im Mai und Juni auftreten. XIA et al. (1993) geben für Qira 19 bis 64 Tage Sandsturm pro Jahr an. Im Untersuchungszeitraum traten 1999 an 73 Tagen (April - Dezember 1999) und 2000 an 81 Tagen Windgeschwindigkeiten über $5 \mathrm{~m}^{*} \mathrm{~s}^{-1}$ auf, davon 68 (1999) bzw. 61 (2000) Tage während der Vegetationsperiode.

Diese Windgeschwindigkeit wird häufig als Grenzwert angegeben (XIA et al. 1993), ab dem sich Schluff zu bewegen beginnt. Dies trägt zur Mobilität der Wanderdünen und zur Versandung von Nutzflächen bei, da Schluff im Untersuchungsgebiet die vorherrschende Korngrößenfraktion ist. Die Wandergeschwindigkeit 
niedriger vegetationsfreier Dünen im Bezirk Qira beläuft sich laut XIA et al. (1993) auf 30 bis $70 \mathrm{~m}$ pro Jahr.

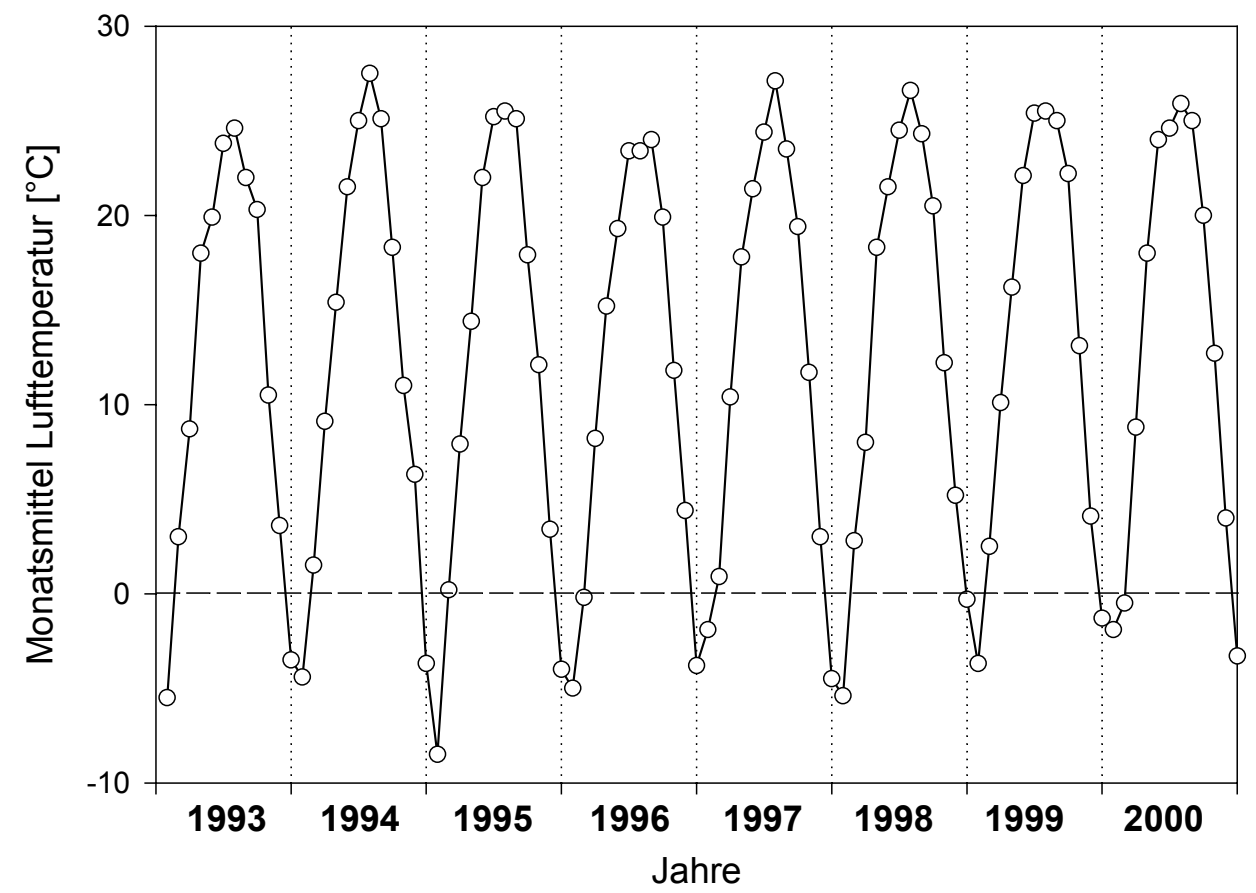

Abb. 2.3.2: Monatsmittel der Lufttemperatur 1993-2000.

Werte der staatlichen Wetterstation in Qira.

\subsection{Boden}

Im Tarimbecken liegen pleistozäne Ablagerungen von mehreren hundert Metern Schichtdicke; lößähnliche lehmige Schluffe kommen in den Randgebieten als mehrere Meter mächtige Schichten vor (WALTER \& BOX 1983). Die Böden im Oasenvorland von Qira sind bis in die im Projekt untersuchte Tiefe von ca. $8 \mathrm{~m}$ sehr einheitlich. Grobschluff ist mit mehr als $87 \%$ die vorherrschende Korngrößenfraktion auf allen Untersuchungsflächen (BRUELHEIDE et al. 2001). Es können weder optisch noch physikalisch verschiedene Horizonte im Bodenprofil voneinander unterschieden werden. Auch chemisch sind die Böden sehr einheitlich. Es wurde nur auf der Alhagi-Fläche ein leicht höherer Ca-Gehalt im Oberboden festgestellt, der vermutlich durch den Streufall der Ca-reichen Alhagistreu dort angereichert wurde. Auf der Tamarix-Fläche wurde ein höherer 
Salzgehalt unter den Tamarix-Büschen gemessen (BRUELHEIDE et al. 2001), der durch den herbstlichen Streufall der Kurztriebe zustande kommt, da T. ramosissima die im Grundwasser enthaltenen Salze $(\mathrm{NaCl})$ über die Blätter abscheidet (KleinKopf \& Wallace 1974, Baum 1978, Glenn et al. 1998).

Im Oasenvorland ist eine ständige Umlagerung und Verwehung von schluffigem Bodenmaterial zu beobachten, besonders an Stellen, an denen die Vegetation entfernt oder aufgelichtet wurde. Als Folge werden auf der Luvseite der Dünen durch Ausblasung alte Sprossteile und Wurzelstöcke freigelegt, während es auf der Leeseite und in dichten Beständen zu Schluffakkumulation kommt. Diese Akkumulation kann bis zu einem Meter pro Jahr betragen (eigene Beobachtungen).

\subsection{Pflanzenarten}

Die untersuchten Schlüsselarten der Vorlandvegetation sind perennierende Pflanzen, die im Untersuchungsgebiet oder in der Region (im Falle von Calligonum caput-medusae) heimisch sind. Es wurden häufig auftretende Pflanzenarten ausgewählt, die von der einheimischen Bevölkerung als Brennholz, Baumaterial oder Viehfutter genutzt werden. In den 1980er Jahren wurde die Nutzung der Vorlandvegetation von den örtlichen Behörden in einem $3 \mathrm{~km}$ breiten Bereich um die Oase verboten, um den Schutz vor Sandverwehung zu gewährleisten.

\section{Alhagi sparsifolia Shap. (Fabaceae)}

Alhagi ist eine taxonomisch kritische Gattung. Heute wird vermutet, dass die Namen A. sparsifolia Shap., A. maurorum Medik., A. pseudalhagi (M. Bieb.) Desv. und $A$. kirghisorum Schrenk. Synonyme sind (B. DICKORÉ, mündliche Mitteilung). Alhagi sparsifolia ist ein dichter dorniger Strauch, der eine Höhe von etwa 1,2 m erreicht (Abb. 2.5.1). In Oasennähe bildet er mehr oder weniger dichte Bestände. Je weiter man in die Wüste vordringt, desto spärlicher tritt $A$. sparsifolia auf. Dort finden sich nur noch vereinzelt Gruppen dieser Pflanze. 
Die oberirdischen Teile des Busches sterben in winterkalten Gebieten mit dem ersten Frost ab, die Nekromasse bleibt aber stehen und wirkt so ebenfalls Sand fixierend. Im Frühjahr erfolgt der Neuaustrieb aus Rhizomen (KASSAS 1952).

Alhagi sparsifolia ist ein Luftstickstoff-Fixierer (ARAMPATSIS 2001). Alhagi ist in vielen Trockengebieten als Weideunkraut bekannt (KERR 1965, RICHARDSON 1953, VAN DER WALT 1955), das mit Herbiziden und mechanischen Maßnahmen bekämpft wird. In Xinjiang aber werden die Büsche ab dem Frühjahr von Schafen, Ziegen und Kamelen beweidet. Im Spätsommer und Herbst wird die ganze Pflanze dicht unter der Bodenoberfläche abgehackt und als Winterfutter geerntet. Außerdem findet $A$. sparsifolia als Gründünger Verwendung.

\section{Calligonum caput-medusae Schrenk. (Polygonaceae)}

Die Gattung Calligonum enthält unregelmäßig wachsende Rutensträucher, d.h. die Blätter sind stark reduziert, die neuen Triebe werden als Assimilationsorgane ausgebildet (Abb. 2.5.2). Die Büsche erreichen in Qira eine Höhe von ca. 3 m.

Die Gattung enthält nur $\mathrm{C}_{4}$-Pflanzen (PYANKov et al. 1994). C. caput-medusae ist nicht indigen im Untersuchungsgebiet, aber weit verbreitet in Zentralasien, und wurde 1985 mit gutem Erfolg als Windschutz in Qira angepflanzt. Versuche zur Regeneration der Wüstenvegetation und zur Sandfixierung wurden mit verschiedenen Arten der Gattung Calligonum durchgeführt: mit Calligonum arborea in Turkmenistan (YARASHOV 1996), mit C. caput-medusae in Usbekistan (FIMKIN et al. 1990) und China (z.B. XIA et al. 1993). Der jährliche Höhenzuwachs kann starke Übersandung ausgleichen (XIA et al. 1993).

Die Büsche werden in Qira vor allem als Brennholz genutzt, da das Holz nach Auskunft der einheimischen Bauern einen guten Brennwert hat. Aber auch als Futterpflanze (SHAMSUTDINOV 1988) und zur Honiggewinnung ist Calligonum bekannt (ZHANG \& MAO 1990). 


\section{Populus euphratica Oliv. (= P. diversifolia, Salicaceae)}

Populus euphratica ist indigen in ganz Zentralasien und darüber hinaus und ist die Hauptbaumart der Tugai-Auwälder (NovikOVA 1987, CoQUE et al. 1991, TIAN 1991). Sie kommt aber auch, wie auf den Untersuchungsflächen in Qira, fernab heutiger Flussläufe in ehemaligen Flussbetten und grundwassernahen Niederungen vor (Abb. 2.5.3). An vielen Flüssen in Xinjiang (z.B. am Unterlauf des Tarim) wird seit Jahren ein Absterben der Populus euphratica Auwälder beobachtet, das auf veränderte Wasserführung der Flüsse und ein Absinken des Grundwasserspiegels zurückgeführt wird (TIAN 1991, OVEZLIEV et al. 1993, TIAN \& SONG 1997). Populus euphratica hat sich auf Grundwasser beeinflussten Standorten in ariden und semi-ariden Gebieten in ganz Mittelasien und Indien zu einer wichtigen forstlich genutzten Baumart entwickelt (LALYMENKO \& LALYMENKO 1990, WANG \& LI 1995, SOOD 1995, SHARMA et al. 1999) und wird häufig als Windschutz angepflanzt (DREGNE 1988, MAKI et al. 1993). ZHAO et al. (1997) zählen $P$. euphratica zu den mäßig salztoleranten Baumarten in China.

$P$. euphratica wurde trotz des häufig unregelmäßigen Wuchses als Baumaterial genutzt, da das Holz sehr witterungsbeständig ist (WANG, CHEN \& LI 1996). Hauptsächlich fand $P$. euphratica als Brennholz Verwendung (MA et al. 1997).

In Xinjiang zählt Populus euphratica zu den geschützten Pflanzenarten (PAN \& YING 1995). Das Schlagen von $P$. euphratica ist seit 1983 im Oasenvorland von Qira verboten, kommt aber noch immer vor (eigene Beobachtungen). Als Brennholz werden heute vor allem subfossile Reste abgestorbener Populuswälder gesammelt. Die Blätter dienen durch Schneiteln der Äste als Viehfutter.

\section{Tamarix ramosissima Ldb. (Tamaricaceae)}

Die Gattung Tamarix ist in Indien, Zentralasien, Nordafrika und Südeuropa heimisch (BAUM 1978) und hat sich in vielen Trockengebieten weltweit ausgebreitet. Tamarix chinensis (als ein Sammelbegriff für die Gruppe aus T. parviflora, $T$. chinensis und $T$. ramosissima) wurde im 19. Jahrhundert in die USA eingeführt und ist etwa seit den 1920er Jahren entlang der Flüsse im trockenen Südwesten häufig (HORTON \& CAMPBELL 1974, EVERITT 1980). T. ramosissima wurde dort seit 
langem als invasive Art behandelt, da sie sich entlang der Flüsse ausgebreitet und die natürliche Vegetation vor allem an gestörten Standorten ersetzt hat (EVERITT 1980). Tamarisken sind als Phreatophyten bekannt (ROBINSON 1958, ANDERSON 1982, Busch \& SMith 1993, SALA, SMith \& DeVITT 1996). Da T. ramosissima in der Lage ist auch aus ungesättigtem Boden Wasser zu beziehen, wurde sie von TURNER (1974) als fakultativer Phreatophyt klassifiziert. Tamarisken machen mengenmäßig den größten Anteil der Vegetation im Oasenvorland von Qira aus. Neben $T$. ramosissima kommen auch $T$. hohenackeri, $T$. laxa und $T$. leptostachys im Untersuchungsgebiet vor (HUDABERDI 2000). T. ramosissima wächst als bis zu $2 \mathrm{~m}$ hoher Strauch in dichten Beständen (Abb. 2.5.4). Durch das Abwerfen der Kurztriebe im Herbst und aufgrund starker Sandakkumulation begünstigt die Pflanze die Dünenbildung (WICKENS 1998, QONG et al. 2002). T. ramosissima kann bis zu einem gewissen Abstand vom Grundwasser mit der Düne emporwachsen (GRIES et al. 2003). Daher sieht man als häufiges Erscheinungsbild von $T$. ramosissima gekrönte Dünen im Oasenvorland. Tamarisken dringen von allen untersuchten Pflanzenarten am weitesten in die Wüste vor. T. ramosissima ist die salztoleranteste (LIU et al. 1984, vergleiche auch GLENN et al. 1998) der untersuchten Pflanzenarten und kann bei einem Salzgehalt von $50 \mathrm{~g}^{\star} \mathrm{I}^{-1}$ wachsen (ZHAO et al. 1997). T. ramosissima kann über Salzdrüsen auf den Blättern die aufgenommenen Salze abscheiden (KLEINKOPF \& WALLACE 1974, WICKENS 1998) und akkumuliert durch das Abwerfen der Kurztriebe im Herbst Salze am eigenen Standort (BRUELHEIDE et al. 2001). Deswegen wird T. ramosissima von KLEINKOPF \& WALLACE (1974) auch als lonenpumpe bezeichnet.

Die längeren jungen Triebe (etwa 2-5 Jahre alt) werden als Baumaterial für Hauswände, Dächer und Zäune benutzt. Dickere Triebe und alte Wurzelstöcke finden bevorzugt Verwendung als Brennmaterial. 

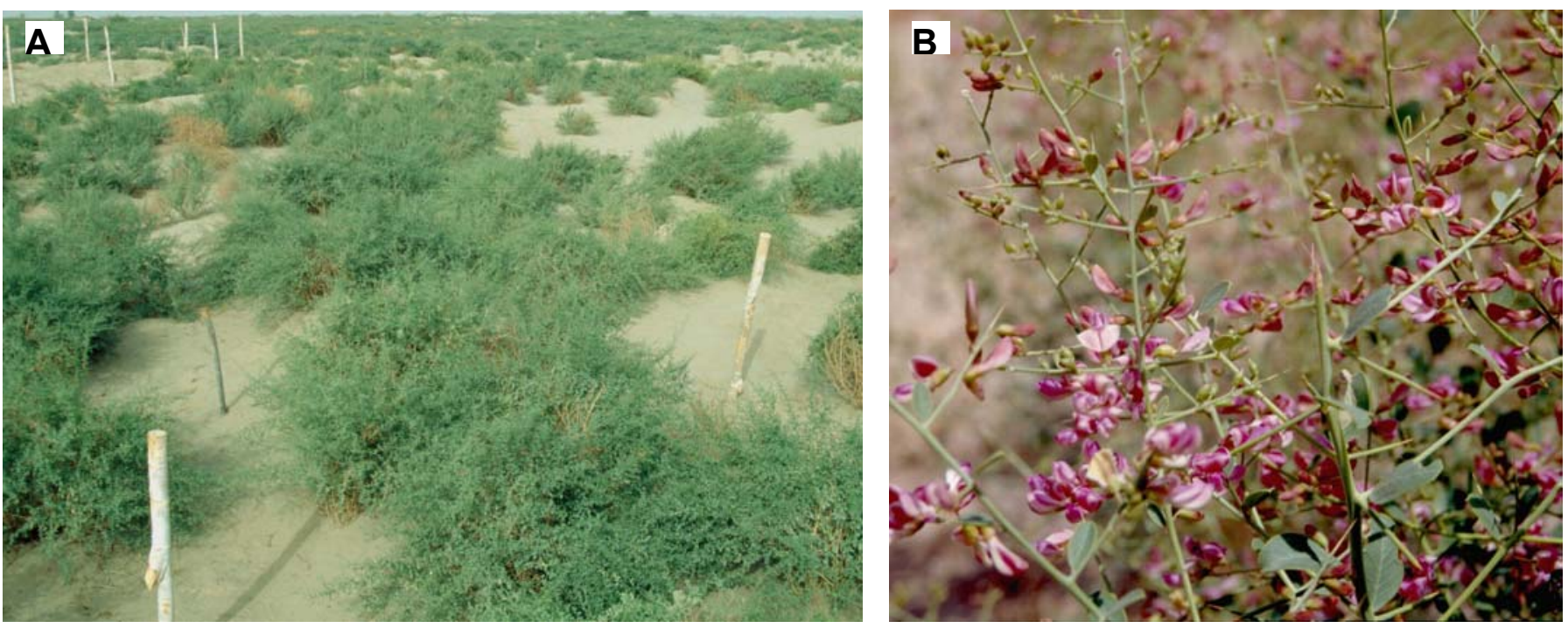

Abb. 2.5.1: Alhagi sparsifolia.

A: Dichter Bestand im Oasenvorland auf der Untersuchungsfläche ADU.

B: Blühender Alhagi sparsifolia im Juni 1999.
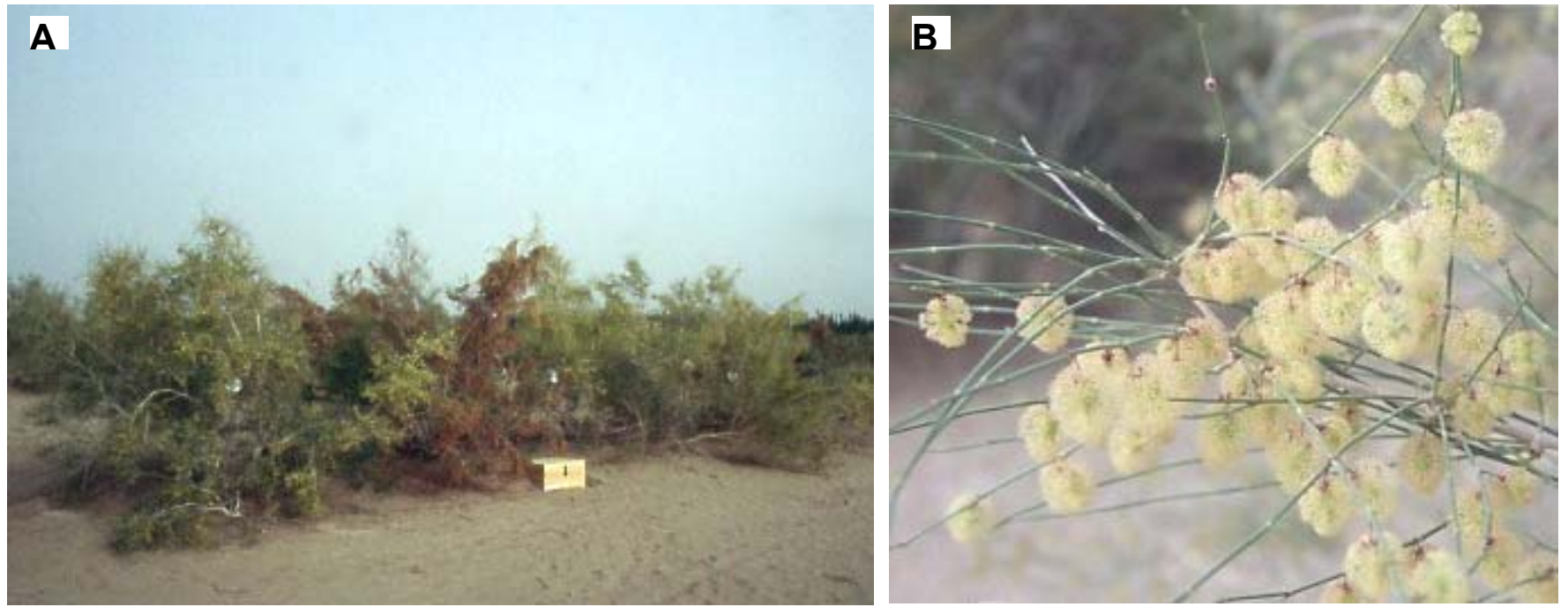

Abb. 2.5.2: Calligonum caput-medusae.

A: Angepflanzte Reihen auf der Untersuchungsfläche CFN.

B: Fruchtender Calligonum caput-medusae im Juni 1999. 

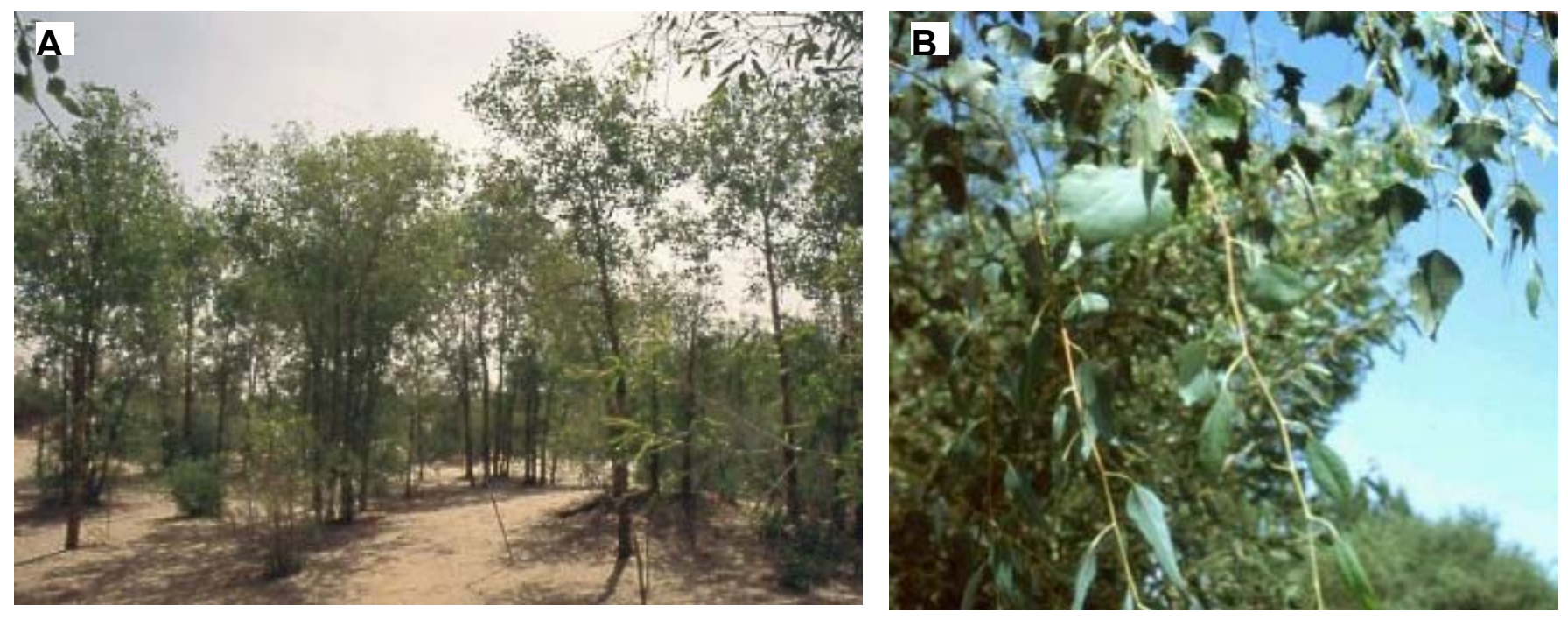

Abb. 2.5.3: Populus euphratica.

A: Lockerer Bestand im Oasenvorland auf der Untersuchungsfläche PDU.

B: Detailaufnahme der verschiedenartig geformten Blätter.
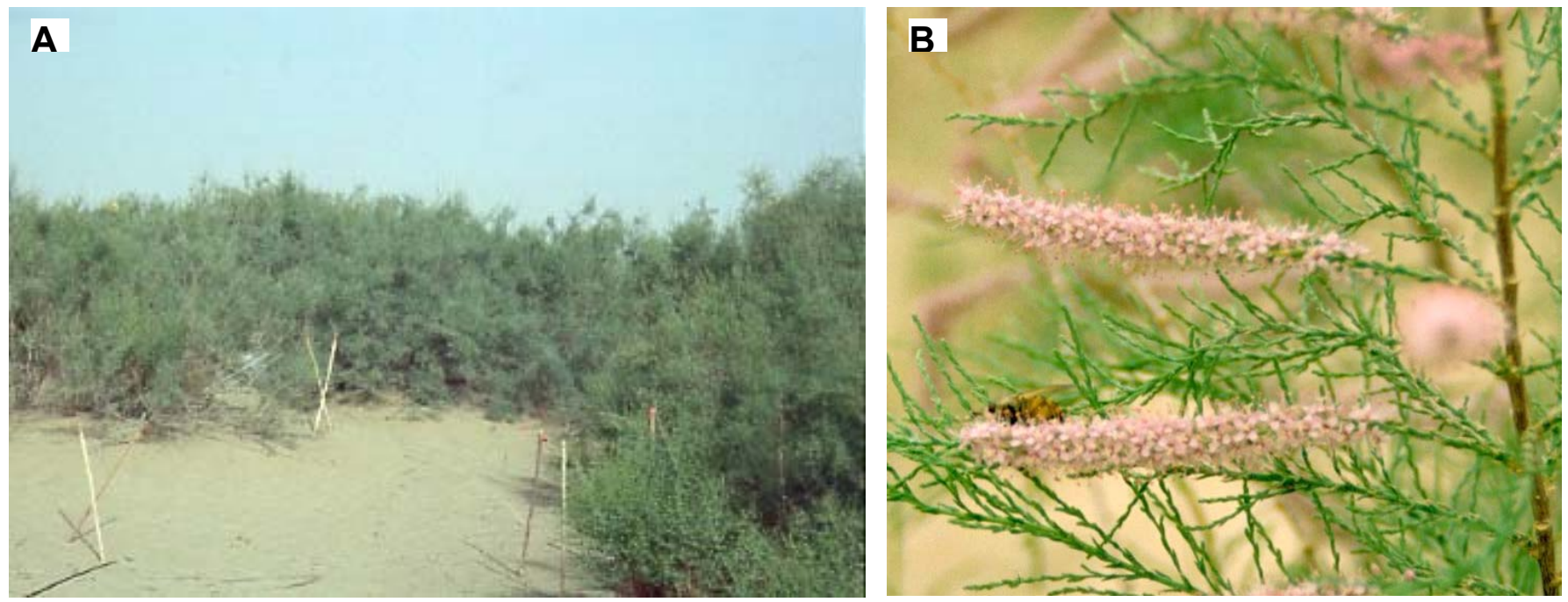

Abb. 2.5.4: Tamarix ramosissima.

A: Dichter Bestand im Oasenvorland auf der Untersuchungsfläche TFN.

B: Blühende Tamarix ramosissima im Juni 1999. 


\section{Material und Methoden}

Die Untersuchungen fanden in einer Forschungsstation der Chinesischen Akademie der Wissenschaften (UTM Koordinaten 475822 / 4096694) in Qira (chinesisch: Cele) am Südrand der Taklamakan statt (Abb. 2.2.1).

Im Oasenvorland wurde ein zweifaktorielles Experiment zur Bewässerung und Nutzung durchgeführt: In Beständen der vier untersuchten Arten wurden Kontrollflächen und Untersuchungsflächen angelegt, die durch eine einmalige Überflutung im Sommer 1999 bewässert wurden. Bodenwassergehalte, Kohlenstoffisotopenverhältnisse und Wasserhaushalt der Pflanzen auf diesen Überflutungsflächen wurden vor der Bewässerung und in der anschließenden Austrocknungsphase untersucht. Einige Parameter wurden als Vergleich ebenfalls auf den unbewässerten Kontrollflächen gemessen. Bestimmungen der Biomassevorräte und des jährlichen Zuwachses wurden auf der Basis von allometrischen Regressionen durchgeführt (GRIES et al. eingereicht). Der Wasserverbrauch auf Blatt- und Bestandesebene wurde durch Transpirations- und Saftflussmessungen bestimmt. Um Effekte der Vegetationsnutzung durch die Bevölkerung zu prüfen, wurde innerhalb des beschriebenen Bewässerungsexperiments die Produktivität von auf ortsübliche Weise genutzten und ungenutzten Beständen bestimmt (GRIES et al. eingereicht). Im Jahr 2000 wurden außerdem Wasserhaushaltsparameter parallel auf den ungenutzten und im Vorjahr experimentell genutzten Untersuchungsflächen gemessen und verglichen.

In China gilt als offizielle Zeit einheitlich die Beijing-Zeit (GMT $+8 \mathrm{~h}$ ), die auch in dieser Arbeit verwendet wurde. Die lokale Xinjiang-Zeit ist um zwei Stunden zeitversetzt (GMT +6 h) und wird in Xinjiang ebenfalls benutzt, jedoch nur inoffiziell. Sonnenhöchststand ist somit 14 Uhr Beijing-Zeit.

\subsection{Witterung}

Die Klimavariablen relative Luftfeuchte $(\mathrm{RH})$, Lufttemperatur, Globalstrahlung, photosynthetisch aktive Strahlung (PAR), Windgeschwindigkeit und Windrichtung 
wurden regelmäßig in beiden Untersuchungsjahren mit zwei Wetterstationen von Campbell Scientific (Shepshed, Leicestershire, UK) mit Standardmethoden gemessen. Eine der beiden Stationen wurde auf der Alhagi-Fläche aufgestellt (Qira_1), die andere auf der Tamarix-Fläche (Qira_2). Die Wetterstationen waren mit einem Logger versehen und wurden über ein Solarpaneel mit Energie versorgt. Gemessen wurde jede Minute, der Mittelwert über zehn Minuten wurde aufgezeichnet. Die aufgenommenen Daten wurden zur Berechnung der Witterungsabhängigkeit der Wasserhaushaltsparameter verwendet.

Aufgrund technischer Probleme wurden im August und September 1999 bei der Wetterstation Qira_1 keine Daten aufgezeichnet. Durch einen Ausfall der Station Qira_2 Ende September entstand eine Datenlücke von 10 Tagen. Für diesen Zeitraum wurden Stundenmittelwerte für relative Luftfeuchte, Lufttemperatur und Sonnenscheindauer von der staatlichen Wetterstation in Qira erworben. Im Winter 1999/2000 wurde nur die Station Qira_1 betrieben, ab April 2000 wieder beide Stationen. Im Sommer 2000 traten wiederum technische Schwierigkeiten bei der Station Qira_1 auf, daher wurden in dieser Zeit die Daten von Qira_2 für alle Berechnungen verwendet. Die Winterdaten stammen wiederum von Qira_1.

\subsection{Untersuchungsflächen}

\subsubsection{Beschreibung der Flächen}

Es wurden im westlichen Oasenvorland Untersuchungsflächen ausgewählt (Abb. 2.1.2), die jeweils von einer der untersuchten Pflanzenarten dominiert wurden. Die Auswahlkriterien für die Flächen waren gleichmäßige Bestandesstruktur und gute Erreichbarkeit. Nahe bei der Forschungsstation wurde für Alhagi sparsifolia eine Fläche am Rand der Oase eingemessen (Abb. 2.5.1), die offensichtlich im vorangegangenen Jahr nicht beerntet worden war, da die Nekromasse vom Vorjahr noch auf der Fläche stand. Auch die von C. caput-medusae dominierte Fläche lag unmittelbar am Oasenrand, da es sich um eine 1985 aus Stecklingen als Windschutz etablierte Pflanzung handelt. Die Bestandesstruktur war in diesem Fall auf der für das Überflutungsexperiment ausgewählten Fläche durch die Pflanzreihen vorgegeben (Abb. 2.5.2). Die nicht bewässerten Flächen lagen auf kleinen 
Dünen. Tamarix ramosissima siedelt auf Dünen bzw. wächst durch Sandakkumulation mit innen empor, daher war es nicht möglich, eine ebene Fläche zu finden. Die Untersuchungsfläche lag einige Kilometer westlich der Oase und bestand aus vegetationsfreien Senken und mit $T$. ramosissima bewachsenen flachen und höheren Dünen (Abb. 2.5.3). Die Populuswälder sind zum Teil offene Galeriewälder entlang der ehemaligen und aktuellen Flussläufe oder finden sich in grundwassernahen Niederungen (Abb. 2.5.4). Sie sind oftmals zusätzlich durch Nutzung aufgelichtet. Die untersuchte Fläche befand sich nahe der Tamarix-Fläche in einem relativ geschlossenen Bestand, der vor etwa 20-25 Jahren aus Stockausschlägen aufgewachsen war und heute eine Höhe von etwa $7 \mathrm{~m}$ erreicht hat. Die Lage der Untersuchungsflächen ist auf dem Satellitenbild des Oasenvorlandes eingezeichnet (Abb. 2.1.2).

Bei Bohrungen auf den Untersuchungsflächen wurden bis in eine Tiefe von $8 \mathrm{~m}$ (Alhagi-Fläche) bzw. 4,5 m (Calligonum-Fläche) praktisch keine Feinwurzeln von A. sparsifolia und C. caput-medusae gefunden. Lebende Feinwurzeln wurden nur auf der Tamarix- und Populus-Fläche im Bereich des Kapillarsaums gefunden, wo der Grundwasserspiegel bei Bohrungen im Frühjahr 1999 bei 5,7 m (T. ramosissima) bzw. 3,6 m (P. euphratica) erreicht wurde.

\subsubsection{Experimentelle Behandlung}

In den vier untersuchten Beständen wurden je zwei ( $P$. euphratica und $T$. ramosissima) oder drei (A. sparsifolia und C. caput-medusae) Flächen von 850 bis $2500 \mathrm{~m}^{2}$ Größe eingerichtet und eingezäunt, zusätzlich gab es eine vegetationsfreie Fläche (Tab. 1). Jeweils eine Fläche pro Pflanzenart wurde im Sommer 1999 einmal künstlich bewässert, die anderen blieben ohne Bewässerung. Für die Bewässerung wurde Wasser aus dem Kanalsystem der Oase (Wasser des QiraFlusses) bis auf die eingedeichten Untersuchungsflächen geleitet, indem die Bewässerungskanäle durch einfache Gräben und Dämme bis ins Oasenvorland verlängert wurden.

Die Menge des Wassers war nicht zu bestimmen, da sie stark vom Wasserstand des Flusses am jeweiligen Tag und der Überflutungsdauer abhing. Die Flächen waren jedoch mindestens für einige Stunden, im Falle von $T$. ramosissima und $P$. 
euphratica fast 24 Stunden, von Wasser bedeckt. Für die Alhagi-Fläche ist eine Berechnung der Wassermenge möglich, da diese Fläche mit Grundwasser aus einem nahe gelegenen Brunnen bewässert werden musste. Aus der Pumpleistung und der Überflutungsdauer lässt sich eine Wassermenge von etwa $0.4 \mathrm{~m}^{3} \mathrm{~m}^{-2}$ errechnen.

Die Alhagi-Fläche wurde am 21. Juni 1999 bewässert, die Calligonum-Fläche am 22. Juli, die Tamarix- und Populus-Fläche am 5. und 6. August und die Freifläche am 9. August 1999.

Innerhalb der bewässerten ( $F$ für flooded) und nicht bewässerten ( $D$ für dry) Flächen wurden zwei Teilflächen (450 bis $850 \mathrm{~m}^{2}$ ) eingerichtet, auf denen zwei Nutzungsvarianten, ungenutzt ( $N$ für not used) und genutzt ( $U$ für used), untersucht wurden. Mit der experimentellen Nutzung sollte die ortsübliche Ernte durch die einheimische Bevölkerung simuliert und ihre Auswirkungen untersucht werden.

Für die unterschiedlichen Behandlungsvarianten und Pflanzenarten wurden zur eindeutigen Beschreibung Flächenkürzel vergeben, die aus jeweils drei Buchstaben bestehen:

Der erste Buchstabe steht für die Untersuchungsfläche:
A $=$ Alhagi sparsifolia
$\mathrm{C}=$ Calligonum caput-medusae
$\mathrm{T}=$ Tamarix ramosissima
$\mathrm{P}=$ Populus euphratica $(=P$. diversifolia)
$\mathrm{O}=$ Freifläche (open ground)

Der zweite Buchstabe steht für das Bewässerungsexperiment:

$\mathrm{F}=$ Bewässerung (flooded)

$\mathrm{D} \quad=\quad$ ohne Bewässerung (dry)

Der dritte Buchstabe steht für das Nutzungsexperiment:
$\mathrm{U}=$ genutzt (used)
$\mathrm{N}=$ ungenutzt (not used)
$\mathrm{R}=$ eingeschränkt genutzt (andere Nutzungsvariante = reduced use, nur bei $A$. sparsifolia) 
Tab. 1: Größe, Lage und Behandlungsvarianten der Untersuchungsflächen.

Die UTM-Koordinaten beziehen sich immer auf die Nordwestecke der eingezäunten Flächen. $\mathbf{F}$ bewässert, $\mathbf{D}$ unbewässert, $\mathbf{N}$ ungenutzt, $\mathbf{U}$ genutzt, $\mathbf{R}$ eingeschränkt genutzt

\begin{tabular}{|c|c|c|c|}
\hline Pflanzenart & Flächenkürzel & Flächengröße & Lage (UTM) \\
\hline $\begin{array}{l}\text { Alhagi sparsifolia } \\
\text { A }\end{array}$ & $\begin{array}{l}\text { AF mit } \\
\text { AFN, AFU, AFR } \\
\text { ADN } \\
\text { ADU und ADR }\end{array}$ & $\begin{array}{l}50 \times 50 \mathrm{~m} \\
(\text { je } 15 \times 50 \mathrm{~m}) \\
30 \times 20 \mathrm{~m} \\
50 \times 30 \mathrm{~m}\end{array}$ & $\begin{array}{l}476246 \text { / } 4097724 \\
476177 \text { / } 4097597 \\
476154 \text { / } 4097680\end{array}$ \\
\hline $\begin{array}{l}\text { Calligonum } \\
\text { caput-medusae } \\
\text { C }\end{array}$ & $\begin{array}{l}\text { CF mit } \\
\text { CFN und CFU } \\
\text { CDN } \\
\text { CDU }\end{array}$ & $\begin{array}{l}50 \times 50 \mathrm{~m} \\
(\text { je } 25 \times 50 \mathrm{~m}) \\
30 \times 15 \mathrm{~m} \\
20 \times 20 \mathrm{~m}\end{array}$ & $\begin{array}{l}478218 \text { / } 4097387 \\
478278 \text { / } 4097562 \\
478274 \text { / } 4097477\end{array}$ \\
\hline $\begin{array}{l}\text { Populus euphratica } \\
\text { P }\end{array}$ & $\begin{array}{l}\text { PF mit } \\
\text { PFN und PFU } \\
\text { PD mit } \\
\text { PDN und PDU }\end{array}$ & $\begin{array}{l}50 \times 20 \mathrm{~m} \\
(\mathrm{je} 25 \times 20 \mathrm{~m}) \\
50 \times 20 \mathrm{~m} \\
(\text { je } 25 \times 20 \mathrm{~m})\end{array}$ & $\begin{array}{l}477542 / 4103260 \\
477486 / 4103411\end{array}$ \\
\hline $\begin{array}{l}\text { Tamarix } \\
\text { ramosissima } \\
\mathbf{T}\end{array}$ & $\begin{array}{l}\text { TF mit } \\
\text { TFN und TFU } \\
\text { TD mit } \\
\text { TDN und TDU }\end{array}$ & $\begin{array}{l}45 \times 40 \mathrm{~m} \\
(\text { je } 45 \times 20 \mathrm{~m}) \\
38 \times 42 \mathrm{~m} \\
(\text { je } 38 \times 20 \mathrm{~m})\end{array}$ & $\begin{array}{l}476989 \text { / } 4103212 \\
476942 \text { / } 4103434\end{array}$ \\
\hline Freifläche $\mathbf{O}$ & OFN & $20 \times 20 \mathrm{~m}$ & $478220 / 4097523$ \\
\hline
\end{tabular}

Die CD- und TD-Flächen befanden sich auf Dünen, da durch die Geländestruktur nur so sichergestellt werden konnte, dass sie von der experimentellen Überflutung ausgespart wurden.

Die Nutzungsernte erfolgte 1999 vom 24.-29. August (A. sparsifolia), 3.-4. September (T. ramosissima), 7.-8. September (C. caput-medusae) und Mitte Oktober (P. euphratica).

Die Behandlung sollte möglichst genau der Art der Nutzung durch die einheimische Bevölkerung (Uighuren) entsprechen, daher wurden für die verschiedenen Pflanzenarten unterschiedliche Erntemethoden angewendet.

Bei A. sparsifolia wurde die gesamte Pflanze mit der von den uighurischen Bauern verwendeten Hacke durch Abhacken des Sprosses etwa $10 \mathrm{~cm}$ unter der Bodenoberfläche entfernt (Flächen AFU und ADU). Bei der zweiten Nutzungsvariante wurden die Sprosse an der Bodenoberfläche abgeschnitten, da auf diese Weise eine größere Zahl der unterirdisch angelegten Erneuerungsknospen erhalten blieb (AFR und ADR).

Bei C. caput-medusae wurde zunächst die stehende Biomassendichte in $2 \mathrm{~m}$ mal $2 \mathrm{~m}$ Probeflächen innerhalb der CFU- und CDU-Fläche bestimmt. Daraufhin wurden einzelne Triebe großer Durchmesser abgeschnitten, bis etwa die Hälfte 
der Biomasse entfernt war. Bei den Büschen in direkter Nachbarschaft der Probeflächen wurden ebenfalls Triebe entfernt, da die Büsche im Kronenraum ineinander griffen und nur so eine Auflichtung auf den Probeflächen sichergestellt war. Auf den Populus-Flächen wurde die Stammzahl um etwa die Hälfte reduziert. Dafür wurde zunächst die Stammdichte auf den für die Nutzung vorgesehenen Flächen (PFU und PDU) ermittelt, um in einem zweiten Schritt in $5 \mathrm{~m}$ mal $5 \mathrm{~m}$ Quadraten so viele Bäume zu fällen, bis die gewünschte Dichte erreicht war. Die Pappeln wuchsen häufig in Gruppen von 2 bis 5 Stämmen, da der Bestand aus Stockausschlägen stammt. Es wurden bevorzugt Bäume aus diesen Gruppen anstatt einzeln stehender Bäume gefällt, um eine gleichmäßigere Verteilung der Stämme auf der Fläche zu erreichen. Auf der nicht bewässerten genutzten Teilfläche (PDU) wurden 66 von 151, auf der bewässerten genutzten 54 von 111 Bäumen gefällt (PFU).

Tab. 2: Bestandesdichte und Begleitarten auf den Untersuchungsflächen 2000.

Die Werte für die Bestandesdichte sind Busch ( $A$. sparsifolia), Baum ( $P$. euphratica) oder Trieb (C. caput-medusae, T. ramosissima) pro $\mathrm{m}^{2}$. Die in Klammern angegebenen Werte stammen von 1999. Bei C. caput-medusae und $T$. ramosissima sind die Bestandesdichten auf die Fläche hochgerechnet (gesamt) sowie nur innerhalb des Bestandes (ohne Freiflächen) angegeben.

\begin{tabular}{|c|c|c|c|}
\hline Pflanzenart & Plot & Bestandesdichte $\left[\right.$ Trieb $\left.^{*} m^{-2}\right]$ & Begleitarten \\
\hline $\begin{array}{l}\text { Alhagi } \\
\text { sparsifolia } \\
\text { A }\end{array}$ & $\begin{array}{l}\text { AFN } \\
\text { AFU } \\
\text { AFR } \\
\text { ADN } \\
\text { ADU } \\
\text { ADR }\end{array}$ & $\begin{array}{l}0,42 \pm 0,08(0,78 \pm 0,13) \\
1,64 \pm 0,19 \\
1,23 \pm 0,18 \\
0,89 \pm 0,08(1,16 \pm 0,10) \\
1,06 \pm 0,19 \\
1,59 \pm 0,16\end{array}$ & $\begin{array}{l}\text { Karelinia caspica, } \\
\text { Launaea } \\
\text { polydichotoma, } \\
\text { Phragmites australis, } \\
\text { Cynanchum acutum }\end{array}$ \\
\hline $\begin{array}{l}\text { Calligonum } \\
\text { caput-medusae } \\
\text { C }\end{array}$ & $\begin{array}{l}\text { CF (gesamt) } \\
\text { CF (im Bestand) } \\
\text { CDN } \\
\text { CDU }\end{array}$ & $\begin{array}{l}3,44 \\
5,91 \\
8,20 \pm 1,81 \\
6,46 \pm 0,81 \\
\end{array}$ & $\begin{array}{l}\text { Launaea } \\
\text { polydichotoma }\end{array}$ \\
\hline $\begin{array}{l}\text { Populus } \\
\text { euphratica } \\
\mathbf{P}\end{array}$ & $\begin{array}{l}\text { PFN } \\
\text { PFU } \\
\text { PDN } \\
\text { PDU }\end{array}$ & $\begin{array}{l}0,28 \\
0,23 \\
0,34 \\
0,25\end{array}$ & $\begin{array}{l}\text { Tamarix ramosissima, } \\
\text { Karelinia caspica }\end{array}$ \\
\hline $\begin{array}{l}\text { Tamarix } \\
\text { ramosissima } \\
\mathbf{T}\end{array}$ & $\begin{array}{l}\text { TF (gesamt) } \\
\text { TF (im Bestand) } \\
\text { TD (gesamt) } \\
\text { TD (im Bestand) }\end{array}$ & $\begin{array}{l}5,71 \\
19,05 \pm 5,42 \\
7,94 \\
18,67 \pm 2,45 \\
\end{array}$ & $\begin{array}{l}\text { Alhagi sparsifolia, } \\
\text { Karelinia caspica, } \\
\text { L. polydichotoma, } \\
\text { Phragmites australis }\end{array}$ \\
\hline
\end{tabular}

Bei $T$. ramosissima war die Anzahl der Triebe zu hoch, um eine ähnliche Auswahl zu treffen, außerdem zeigte sich innerhalb der Bestände eine gleichmäßige 
Struktur und Triebdichte. Daher wurden auf den Flächen TFU und TDU in $1 \mathrm{~m}$ breiten Streifen alle Triebe abgeschnitten und jeweils Streifen von $1 \mathrm{~m}$ Breite dazwischen stehen gelassen.

Im Falle von C. caput-medusae (CF-Fläche) und T. ramosissima sind wegen der ungleichmäßigen Bestandesstruktur die Dichten sowohl für die gesamte Fläche (einschließlich der vegetationsfreien Bereiche) als auch für die angepflanzten Reihen (bei C. caput-medusae) bzw. innerhalb der Bestände (bei T. ramosissima) angegeben.

\subsection{Bodenwassergehalt}

Der volumetrische Bodenwassergehalt (Vol. \%) wurde mit TDR-Sonden (TDR = Time Domain Reflectometry) während der Vegetationsperiode gemessen.

Die Sonden wurden mit Hilfe eines Handbohrgerätes auf den FN-Flächen eingesetzt. In den Bodentiefen 20-30 cm, 90-100 cm, 190-200 cm und 340-350 cm wurden Sonden des Typs TRIME-FM2/P2 (IMCO, Ettlingen, Deutschland) verwendet, deren maximale Messtiefe $350 \mathrm{~cm}$ beträgt. In den größten erreichten Tiefen (460 bis $775 \mathrm{~cm}$, siehe Tab. 3) wurden Sonden des Typs TRIME-IT (IMCO, Ettlingen, Deutschland) eingesetzt, die bis zu $15 \mathrm{~m}$ Tiefe verwendet werden können.

Tab. 3: Messtiefe und Typ der TDR-Sonden auf den Untersuchungsflächen.

Die Flächenkürzel stehen für A Alhagi sparsifolia, C Calligonum caput-medusae, T Tamarix ramosissima, $\mathbf{P}$ Populus euphratica, $\mathbf{O}$ vegetationsfreie Fläche (open ground), $\mathbf{F}$ bewässert (flooded), $\mathbf{N}$ ungenutzt (not used).

\begin{tabular}{l|l|l|l|l|l}
\hline \multicolumn{7}{|c}{ Messtiefe der TDR-Sonden [cm] } \\
\hline Sondentyp & AFN & CFN & TFN & PFN & OFN \\
\hline TRIME-FM2 & $20-30,90-100,190-200,340-350$ & & \\
\hline TRIME-IT & $750-760$ & $450-460$ & $650-660$ & $456-466$ & $765-775$ \\
\hline
\end{tabular}

Die unterschiedlichen maximalen Tiefen der TDR-Sonden ergaben sich aus der Beschaffenheit der Untersuchungsflächen. Auf der Alhagi-Fläche und der Freifläche konnte ungehindert gebohrt werden, bis die maximale Länge des Bohrgestänges erreicht war. Auf der Tamarix- und Populus-Fläche wurde in einer Tiefe von 5,0 bzw. 2,7 m der Kapillarsaum des Grundwasser erreicht. Als eine 
wassergesättigte Bodenschicht erreicht worden war, wurde noch etwa einen Meter tiefer gebohrt, um eventuelle Schwankungen des Grundwassers zu erfassen. Auf der Calligonum-Fläche wurde das Vordringen in größere Tiefen durch das Auftreten von festen, mit dem vorhandenen Gerät undurchdringlichen Bodenschichten verhindert, daher liegt dort die tiefste TDR-Sonde in nur $450-460 \mathrm{~cm}$.

Im Jahr 2000 wurde auf der Tamarix-Fläche eine zusätzliche TDR-Sonde des Typs TRIME-IT in 587-597 cm Bodentiefe eingesetzt. Am Ende der Vegetationsperiode wurden auf allen Untersuchungsflächen die Sonden in 20-30 cm Bodentiefe entfernt, um Frostschädigungen zu vermeiden. Im folgenden Frühjahr wurden die Sonden wieder eingesetzt.

Der volumetrische Wassergehalt des Bodens wurde in beiden Untersuchungsjahren in wöchentlichem Abstand mit einem Handauslesegerät gemessen. Um die rasche Änderung des Bodenwassergehalts nach der experimentellen Überflutung 1999 zu erfassen, wurde die Auslesefrequenz auf dreimal wöchentlich in der ersten Woche und zweimal wöchentlich in der folgenden Woche erhöht.

\subsection{Entwicklung der Blattfläche und des Blattflächenindexes (LAI)}

Um blattgewichtsbezogene Wasserhaushaltsparameter berechnen zu können wurden monatlich Ernten der transpirierenden Organe durchgeführt. Im Folgenden wird der Begriff „Blatt“ für die transpirierenden Organe aller untersuchten Arten verwendet, ebenso die Begriffe Blattmasse oder Blatttrockengewicht. Mit diesen Erntedaten wurde die Entwicklung der Blattmasse im Jahresverlauf modelliert. Auf der Basis von Geländebeobachtungen wurde der 1. April als Beginn der Blattentwicklung notiert.

Von Mai bis September 1999 wurden von A. sparsifolia monatlich 10-12 Büsche unterschiedlicher Größe komplett geerntet und bei $60^{\circ} \mathrm{C}$ für 48 Stunden getrocknet und gewogen. Von C. caput-medusae und T. ramosissima wurden monatlich 10-15 Triebe unterschiedlicher Durchmesser (etwa 2-40 mm) geerntet, nach Holz und transpirierenden Organen (Blätter, Kurztriebe) getrennt und ihr Trockengewicht bestimmt. Vor dem Trocknen wurden von allen Blattproben Teilproben genommen, diese zur Bestimmung der transpirierenden Flächen gescannt und anschließend ebenfalls bei $60^{\circ} \mathrm{C}$ getrocknet und gewogen. Die Bestimmung der 
Blattfläche erfolgte mit dem Programm Delta-T SCAN Version 2.04nc (Delta-T Devices Ltd., UK). Aus den Durchmessern der geernteten Triebe und den ermittelten Blattmassen wurden Jahresverläufe der Blattentwicklung berechnet. Der Erntetermin, an dem die höchste Biomasse gemessen wurde (bei allen Arten im August), wurde als $100 \%$ gesetzt und die Biomasse der übrigen Termine als relative Werte gegenüber dem Augustwert umgerechnet. Der relative Wert wurde gewählt, da bei $C$. caput-medusae und $T$. ramosissima der Durchmesser einen stärkeren Einfluss auf die Blattmasse hatte als der Termin. An diese Wertepaare (Juliantag/relative Blattmasse) wurden Kurven angepasst.

Auch 2000 wurden wieder monatliche Ernten von A. sparsifolia, C. caput-medusae und $T$. ramosissima durchgeführt. Auf die Ermittlung der Blattfläche sowie der Holzmasse wurde verzichtet. Es wurde nur der Triebdurchmesser und das Blatttrockengewicht bestimmt.

Bei $P$. euphratica wurde in beiden Untersuchungsjahren an Stelle einer Ernte monatlich die relative Beleuchtungsstärke im Bestand gemessen. Hierfür wurde auf der PF- und der PD-Fläche im Abstand von $5 \mathrm{~m}$ mal $5 \mathrm{~m}$ mit markierten Stäben ein Raster abgesteckt, an dessen Schnittpunkten in etwa $1 \mathrm{~m}$ Höhe mit einem PAR-Sensor (LI 1000, LI-COR, Lincoln, Nebraska, USA) die Strahlung gemessen wurde. So ergaben sich 40 Messpunkte pro Untersuchungsfläche. Als Referenz diente ein baugleicher Sensor außerhalb der Fläche unter freiem Himmel. Es wurde nur an Tagen mit gleichmäßig bedecktem Himmel zwischen 11 und 14 Uhr Beijingzeit gemessen.

Diese Messung erfolgte auch am Tag vor einer Ernte von 15 Pappeln unterschiedlichen Durchmessers im August 1999. Für beide Untersuchungsflächen wurde aus der Messung der relativen Beleuchtungsstärke zum Zeitpunkt der Ernte und dem aus allometrischen Gleichungen und Stammdurchmessern berechneten Blattflächenindex nach dem Lambert-Beer'schen Gesetz ein Extinktionskoeffizient k berechnet:

$$
\frac{i}{i 0}=\exp \left(-k^{*} L A I\right)
$$

i Beleuchtungsstärke im Bestand

i0 Beleuchtungsstärke im Freiland

k Kombination aus Blattprojektionskoeffizient und Absorptionskoeffizient

LAI Blattflächenindex $\left[\mathrm{m}^{2 *} \mathrm{~m}^{-2}\right]$ 
Mit dieser Beziehung wurde der LAl für verschiedene Zeitpunkte berechnet. An die Wertepaare (Juliantag und LAI) wurde eine Exponentialfunktion angepasst.

\subsection{Wasserpotential}

Die Messung des Wasserpotentials $\Psi$ erfolgte nach der Druckkammermethode von SCHOLANDER, durch die das Druckpotential im Xylem (Apoplasten) bestimmt wird (SCHOLANDER et al. 1965). Da der osmotische Wert und das Matrixpotential des Apoplasten in der Regel vernachlässigbar sind (TYREE und JARVIS 1982), kann der Xylem-Messwert dem apoplastischen Wert des Blattwasserpotentials gleichgesetzt werden. Befindet sich das Gewebe im thermodynamischen Gleichgewicht, so sind die Wasserpotentiale des Apoplasten und des Symplasten gleich groß. Ein Gleichgewicht stellt sich im Augenblick des Abschneidens des Zweiges oder Blattes ein, wenn die Transpiration gegen Null geht, und ein Ausgleich der Wasserpotentiale zwischen Apoplast und Symplast erfolgt (KoIDE et al. 1989). Dabei wird angenommen, dass der Wassergehalt des Apoplasten während der Messung konstant bleibt.

Die Wasserpotentialmessungen wurden mit einer SCHOLANDER-Bombe der Firma PMS Instruments (Corvallis, Oregon, USA) durchgeführt. Es wurden die Wasserpotentiale von Trieben bzw. Zweigen aus dem mittleren Kronenbereich gemessen: bei $P$. euphratica in etwa $4 \mathrm{~m}$, bei $T$. ramosissima und $C$. caput-medusae in etwa 1-2 $\mathrm{m}$ und bei $A$. sparsifolia in etwa 0,6-0,8 $\mathrm{m}$ Höhe. Die Proben wurden mit einer Rasierklinge abgetrennt und sofort in die Druckkammer eingesetzt.

Die gemessenen Triebabschnitte waren bei $A$. sparsifolia etwa $20 \mathrm{~cm}$, bei den anderen Arten etwa $15 \mathrm{~cm}$ lang und hatten mindestens fünf Blätter bzw. transpirierende Kurztriebe.

1999 wurden von allen vier Pflanzenarten monatlich predawn-Wasserpotentiale $\left(\Psi_{p d}\right)$ und Tagesgänge mit Messpunkten im Abstand von 1-2 Stunden auf den FNFlächen gemessen. Es wurden jeweils sechs Pflanzen mit drei Parallelen beprobt. Im Jahr 2000 wurden nur predawn- und Mittagswasserpotentiale (midday-Wasserpotentiale, $\left.\Psi_{\mathrm{md}}\right)$ an je vier Pflanzen mit drei Parallelen pro Fläche gemessen. Bei Alhagi sparsifolia wurde nur auf der AFN-Fläche gemessen, weil durch die 
Nutzung keine Änderung im Wasserhaushalt erwartet wurde, bei den anderen drei Arten parallel auf den FN- und FU-Flächen.

Die Messungen erfolgten in beiden Untersuchungsjahren an den gleichen Tagen wie die Transpirationsmessungen.

\subsection{Transpiration und stomatäre Leitfähigkeit}

Die Transpiration auf Blattebene und die stomatäre Leitfähigkeit für Wasserdampf wurden mit dem Steady State Porometer LI-1600 der Firma LI-COR (Lincoln, Nebraska, USA) gemessen. Für $P$. euphratica wurde die „broad leaf chamber" verwendet, die eine ebene Blattfläche von $2 \mathrm{~cm}^{2}$ für die Messung erfordert. Für $C$. caput-medusae und $T$. ramosissima dagegen wurde die "conifer chamber" verwendet, da diese beiden Arten nur reduzierte Blätter aufweisen und mit ihren Sprossen bzw. Kurztrieben Photosynthese betreiben. Auch A. sparsifolia wurde, bis auf den ersten Messtermin 1999, mit der Koniferenkammer gemessen, obwohl Blätter in der passenden Größenordnung vorhanden gewesen wären, da auch die ebenfalls Photosynthese treibenden Sprosse und Dornen erfasst werden sollten.

Bei der Messung wird die relative Luftfeuchte der umgebenden Luft als Sollwert eingestellt, dann das transpirierende Organ der Pflanze in die Messkammer eingespannt und so lange getrocknete Luft in die Kammer geleitet, bis wieder die gleiche relative Lufffeuchte wie in der Außenluft erreicht ist. Unter diesen Gleichgewichtsbedingungen (steady state) kann aus der Durchflussrate der trockenen Luft über die Wasserdampfpartialdruckdifferenz zwischen Blatt und Luft die stomatäre Leitfähigkeit und die Transpiration errechnet werden. Die Wasserdampfpartialdruckdifferenz wird über die Luft- und Blatttemperaturen und die relative Luftfeuchte berechnet.

Es wurden in beiden Untersuchungsjahren pro Pflanzenart 6 Individuen mit drei Parallelen auf den FN-Flächen ausgesucht, an denen einmal monatlich die Transpiration im Tagesgang gemessen wurde. 1999 wurden im Sommer ebenfalls 6 Individuen auf den DN-Flächen ausgewählt, an denen im August und September die Transpiration gemessen wurde. Im Jahr 2000 wurden parallel zur Messung auf den FN-Flächen 6 Individuen der FU-Flächen untersucht. Am Ende des Tages wurden jeweils die Kurztriebe oder Blätter, an denen der Tagesgang gemessen 
worden war, geerntet und in einer Plastiktüte gegen Austrocknung geschützt ins Labor gebracht. Dort wurden die Blattflächen durch Scannen und anschließende Berechnung mit dem Programm Delta-T SCAN Version 2.04nc (Delta-T Devices Ltd., UK) ermittelt, die Proben anschließend bei $60{ }^{\circ} \mathrm{C}$ getrocknet und gewogen.

Es wurde nicht an denselben Trieben oder Pflanzen Transpiration und Saftfluss gemessen, außer bei $P$. euphratica, da angenommen wurde, dass hier die Beeinflussung des Saftflusses durch die Entnahme eines Blattes nur gering sein würde.

\subsection{Xylemsaftfluss}

Die einzigen Massenflüsse in verholzten Trieben/Stämmen sind Xylem- und Phloemsaftfluss. Da die Flussrate des Phloemsaftes vernachlässigbar gering gegenüber der des Xylemsaftes ist, kann der gemessene Wasserfluss im Stamm dem Xylemsaftfluss gleichgesetzt werden (PEARCY et al. 1989).

\subsubsection{Methode nach KUČERA}

Für A. sparsifolia, C. caput-medusae und T. ramosissima wurde das Baby Sap Flow System der Firma EMS (Environmental Measuring Systems, Brno, Tschechische Republik) verwendet. Es kann an Trieben mit einem Durchmesser von 12$18 \mathrm{~mm}$ installiert werden. Die Spitzen der Sensoren (Thermoelemente) werden im Abstand von $15 \mathrm{~cm}$ etwa $0,5 \mathrm{~cm}$ tief in das Holz gedrückt. Um die beiden Sensoren liegt eine Schaumstoffhülle, die mit Aluminiumblech umschlossen ist. Die obere Sensorspitze wird durch ummantelte Heizdrähte konstant beheizt (,heat balance"-Methode). Die Saftflussmenge wird durch die Heizspannung berechnet, die zur Aufrechterhaltung einer konstanten Temperaturdifferenz zwischen beheiztem und unbeheiztem Sensorteil benötigt wird. Sie wird von einem Datalogger (sap flow meter T693.2) aufgezeichnet und mit der mitgelieferten Software in Saftflussraten umgerechnet. Aus den minütlichen Messwerten wurde der Mittelwert von 15 Minuten berechnet und aufgezeichnet. Um Störungen durch Temperaturschwankungen zu vermeiden, werden die gesamten Sensoren mit einer aus aluminiumbeschichteter Plastikfolie bestehenden Hülle abgeschirmt. 
Bei Calligonum caput-medusae und Tamarix ramosissima wurden die Sensoren in ca. $1 \mathrm{~m}$ bzw. 30-40 cm über dem Boden installiert. Bei Alhagi sparsifolia wurden die Sensoren unterirdisch (etwa $20-30 \mathrm{~cm}$ Bodentiefe) angebracht, da die oberirdischen Triebe zu geringe Durchmesser aufwiesen. Daher wurde in diesem Fall der Saftfluss einer ganzen Pflanze gemessen. Bei A. sparsifolia wurde die Messanordnung zusätzlich von einer stabilen Plastikfolie geschützt, um den Kontakt mit dem Boden zu vermeiden. Der Saftfluss wurde auf den bewässerten ungenutzten Flächen (FN) an je 6 Trieben bzw. Pflanzen pro Art gemessen.

\section{Modellierung des Saftflusses von A. sparsifolia, C. caput-medusae und T. ramosissima (System nach KUČERA)}

Im Verlauf der Vegetationsperiode traten größere und kleinere Datenlücken auf, die oft durch Ausfälle der Geräte (defekter Logger, zu geringe Batteriespannung, Abbau der Geräte während der experimentellen Überflutung) bedingt waren. Andere Datenlücken sind darauf zurückzuführen, dass die Heizleistung der im Logger integrierten Heizung offensichtlich zu gering war, um bei den hohen auftretenden Saftflussraten volle Funktionstüchtigkeit zu gewährleisten. Letzteres führte zu Datenausfällen um die Mittagszeit (Abb. 3.7.1), die auftraten, sobald eine Thermospannung von etwa $72 \mathrm{mV}$, also ein Saftfluss von etwa $200 \mathrm{~g}^{*} \mathrm{~h}^{-1}$, überschritten wurde.

Um diese Datenlücken zu schließen wurde versucht, eine klare Beziehung zwischen gemessenen Saftlussraten und gemessenen Witterungsparametern festzustellen. Trägt man die relative Luftfeuchte, die photosynthetisch aktive Strahlung (PAR), die Lufttemperatur oder das Wasserdampfsättigungsdefizit der Luft (VPD) gegen die Saftflussraten auf, so erhält man Punktwolken. Um diese wurden Hüllkurven gelegt, die meist mit einem exponentiellen Anstieg zum Maximum beschrieben werden konnten. Für die einzelnen Klimaparameter wurde der erwartete Saftlluss mit den Kurvengleichungen dieser Hüllkurven berechnet. Von den berechneten Werten wurde der für einen gegebenen Zeitpunkt niedrigste Wert herausgesucht, um den Saftfluss zu modellieren. So wurde der für den Saftfluss zu diesem Zeitpunkt limitierende Klimafaktor zur Beschreibung 
herangezogen. Mit diesem Modell erhält man allerdings viel zu hohe Saftllussraten, es wurde daher verworfen.

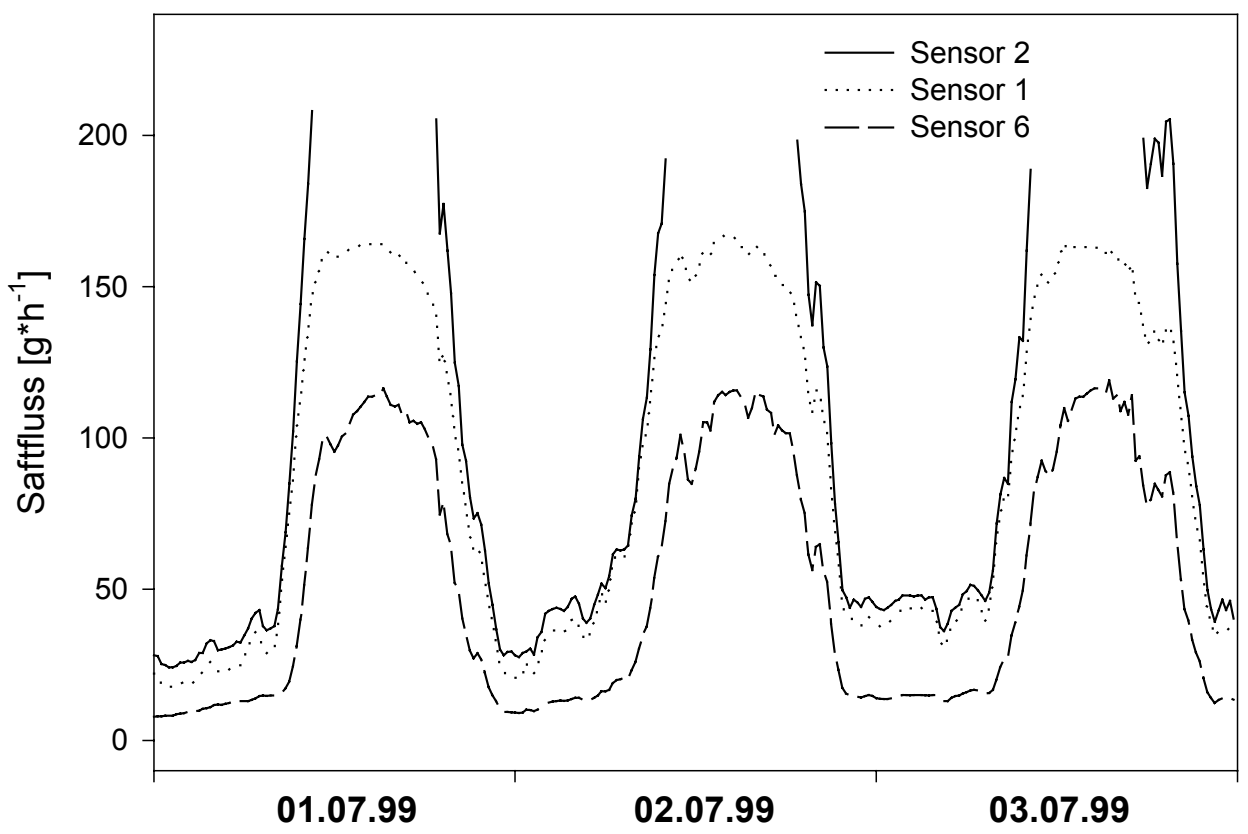

Abb. 3.7.1: Tagesgänge des Saftflusses von Alhagi sparsifolia mit Mittagslücken. Dargestellt sind 3 Sensoren in der Zeit vom 01.07. bis 03.07.1999.

Einzig die Globalstrahlung zeigt eine klarere Beziehung zum Saftfluss (Abb. 4.6.2). Trägt man die Globalstrahlung gegen den Saftfluss auf, kann mit den ermittelten Regressionsgleichungen der Saftfluss modelliert werden. Da sich das Verhältnis von Globalstrahlung zum Saftlluss im Jahresverlauf verändert, wurde für verschiedene Zeitabschnitte, etwa für jeden Monat, eine neue Beziehung berechnet, indem die Strahlung gegen die Saftflussrate des Sensors mit den wenigsten Datenlücken aufgetragen wurde. Das so entstandene Berechnungsmodell zeigte eine durchschnittliche Abweichung von maximal $13,3 \%$ bei $A$. sparsifolia (siehe Kap. 4.6.1).

Dieses Modell ergibt bei der Berechnung der nächtlichen Saftflusswerte einen fast konstanten Wert, da es auf der Strahlung beruht. Dies entspricht nicht der Realität. Da für die meisten Tage aber nur eine Modellierung für die mittags auftretenden Messlücken gebraucht wurde und nachts die Sensoren funktionierten, war das Modell ausreichend genau. An Tagen mit nächtlichen Messausfällen konnte für die Berechnung des Saftflusses auch das Verhältnis der Sensoren untereinander 
herangezogen werden. An komplett modellierten Tagen zeigt sich, wenn man nur die Tagessumme des Saftflusses betrachtet, dass die Überschätzung der Werte nachts durch eine Unterschätzung der Werte mittags ausgeglichen wird (siehe Kap. 4.6.1).

Da das Verhältnis der Sensoren untereinander in den einzelnen Monaten sehr konstant ist, konnte die modellierte Saftflussrate auf die anderen Sensoren umgerechnet werden (siehe Beispiel im Ergebnisteil). Sämtliche verwendeten Formeln mit Regressionskoeffizienten und Irrtumswahrscheinlichkeiten sind im Anhang (Tabellen $1-4,7,8$ ) dargestellt.

\subsubsection{Methode nach GRANIER}

Für Populus euphratica wurde das System der Firma UP (UP Umweltanalytische Produkte, Ibbenbüren, Deutschland) verwendet. Die Datenerfassung erfolgte mit dem Logger Skye Datahog 2 (skye Instruments Ltd., Powys, UK). Gemessen wurde jede Minute, der Mittelwert über 10 Minuten wurde gespeichert.

Ein Messelement dieses Saftflusssystems besteht aus zwei Kupfer-KonstantanThermoelementen mit einer Kupferheizwicklung. Die Sensoren wurden im Abstand von $15 \mathrm{~cm}$ übereinander in etwa 1 bis 1,3 m Höhe am Stamm installiert. Der obere Sensor wurde mit einer konstanten Stromstärke beheizt. Durch den Saftfluss verändert sich die Temperaturdifferenz zwischen unbeheiztem und beheiztem Sensor. Diese Temperaturdifferenz diente als Berechnungsgrundlage für den Saftfluss. Die Xylemflussdichte wurde nach folgender Formel berechnet:

$$
\begin{aligned}
& u=0,714 *\left(\left(\frac{d T_{\text {Nacht }}}{d T_{\text {Aktuell }}}\right)-1\right)^{1.231} \quad\left[\mathrm{ml}^{*} \mathrm{~cm}^{-2 *} \mathrm{~min}^{-1}\right] \\
& \mathrm{u} \quad=\text { Xylemflussdichte } \\
& \mathrm{dT}_{\text {Nacht }}=\text { Differenztemperatur bei Nacht } \\
& \mathrm{dT}_{\text {Aktuell }}=\text { Differenztemperatur bei Messung }
\end{aligned}
$$

Dann wurde von den Messbäumen der leitende Querschnitt bestimmt, um mit diesen Werten den Saftfluss zu berechnen. Dazu wurden die Bäume abgesägt, 
unter Wasser nachgeschnitten und die Stammabschnitte, an denen das Messsystem angebracht war, umgekehrt aufgehängt. Eine Manschette aus Moosgummi wurde aufgesetzt, am Stamm dicht abschließend befestigt und mit einer Fuchsinrot-Lösung gefüllt. Nach einigen Stunden waren die leitenden Flächen durch die der Schwerkraft folgenden Lösung rot angefärbt und konnten mit einer Schieblehre vermessen werden. Die leitende Querschnittsfläche geht wie folgt in die Berechnung des Saftflusses ein:

$$
\begin{array}{rl}
F=u^{*} & S A \quad\left[\mathrm{ml}^{*} \mathrm{~min}^{-1}\right] \\
\mathrm{F} \quad= & \text { Xylemsaftfluss } \\
\mathrm{SA} \quad= & \text { Querschnitt des Splintholzes in Höhe des warmen } \\
& \text { Messfühlers }\left[\mathrm{cm}^{2}\right]
\end{array}
$$

Der Saftfluss wurde an 6 Bäumen auf der bewässerten ungenutzten Fläche (PFN) gemessen.

\section{Modellierung des Saftflusses von P. euphratica (System nach GRANIER)}

1999 war im Zeitraum von April bis Ende Juli eine Heizung defekt, daher konnten nur drei Sensoren betrieben werden. Ab Mitte Juni waren die Sensoren durch den stark salzhaltigen Xylemsaft (LIU JIAJIN, unveröffentlicht) der Pappeln korrodiert, außerdem gab es Probleme beim Auslesen der Daten, so dass im Zeitraum von Mitte Juni bis Ende Juli eine Datenlücke entstand, für die es keine Möglichkeit der Modellierung gab. Ab Anfang September war der Datalogger defekt. Für 1999 liegen daher nur lückenhafte Messungen vor. Kleinere Messlücken konnten mit Beziehungen zwischen VPD und Saftfluss oder dem Verhältnis der Sensoren untereinander geschlossen werden. Es wurden für die einzelnen Sensoren zu verschiedenen Tageszeiten (vormittags, nachmittags, nachts) für jeden Monat das VPD gegen die Temperaturdifferenz bei der Saftflussdichte aufgetragen und mittels linearer Regression die Beziehung ermittelt.

Im Jahr 2000 liefen bei $P$. euphratica nur zwei Sensoren. An sehr vielen Tagen fielen die Sensoren in der Nacht oder am frühen Morgen aus und lieferten erst ab Mittag wieder Messwerte. In jedem Monat wurden die Tage, an denen die 
Sensoren funktionierten, ausgewählt und das VPD gegen die Temperaturdifferenz aufgetragen. Dafür wurde der Abschnitt von 7 bis 12 Uhr ausgewählt, da in diesem Zeitraum für die anderen Tage keine Messwerte vorlagen. Die mittels linearer Regression erhaltenen Beziehungen konnten verwendet werden, um die fehlenden Werte zu modellieren. Es wurde die errechnete Beziehung der Tage verwendet, die zeitlich am nächsten zu den lückenhaften Tagen lagen und ein ähnlich hohes VPD aufwiesen.

Alle verwendeten Formeln sind im Anhang (Tab. 5 und 6) mit Regressionskoeffizienten und Irrtumswahrscheinlichkeiten dargestellt.

\subsection{Wassernutzungseffizienz}

Die Wassernutzungseffizienz der Produktion (water use efficiency of production, WUEp) ist der Quotient aus der verbrauchten Wassermenge und der daraus gebildeten Biomasse. Als verbrauchte Wassermenge wurde die Summe des Xylemsaftllusses über die Vegetationsperiode bestimmt. Als gebildete Biomasse wurde die oberirdische Produktion des jeweiligen Jahres angenommen.

$$
\text { WUEp }=\frac{\text { oberirdische Produktion }[\mathrm{g} \mathrm{TG}]}{\text { Xylemsaftflussumme }\left[\mathrm{kg} \mathrm{H}_{2} \mathrm{O}\right]}
$$

Die Wassernutzungseffizienz wurde auf die Fläche (pro Hektar) bezogen berechnet.

Wegen lückenhafter Saftflussmessungen konnte 1999 nicht für $P$. euphratica und 2000 wegen Ausfall der Logger auf den anderen Probeflächen nur für $P$. euphratica, aber nicht für die anderen Arten die WUEp berechnet werden. 


\subsection{Kohlenstoff-Isotopen-Analysen $\left(\delta^{13} \mathrm{C}\right)$}

Neben dem Kohlenstoffisotop ${ }^{12} \mathrm{C}$ kommt mit einer natürlichen Häufigkeit von $1,1 \%$ auch das schwerere Isotop ${ }^{13} \mathrm{C}$ vor (VOET \& VOET 1992). Pflanzen diskriminieren gegen ${ }^{13} \mathrm{CO}_{2}$. Zunächst wird der Anteil an ${ }^{13} \mathrm{CO}_{2}$ bei der Diffusion durch die Stomata reduziert. Diese Diskriminierung basiert auf der Differenz der Diffusionskoeffizienten von ${ }^{13} \mathrm{CO}_{2}$ und ${ }^{12} \mathrm{CO}_{2}$, der auf dem unterschiedlichen Molekulargewicht beruht. Dadurch entsteht eine Verarmung von ${ }^{13} \mathrm{C}$ gegenüber der atmosphärischen Konzentration.

Eine biochemische Diskriminierung tritt bei $\mathrm{C}_{3}$-Pflanzen auf, da Ribulose-1,5-bisPhosphat-Carboxylase-Oxygenase (RubisCO) eine geringere Affinität für ${ }^{13} \mathrm{CO}_{2}$ verglichen mit ${ }^{12} \mathrm{CO}_{2}$ aufweist. $\mathrm{C}_{3}$-Pflanzen enthalten dadurch deutlich weniger ${ }^{13} \mathrm{C}$ als ihre anorganische Umwelt (O'LEARY 1988). $\mathrm{C}_{4}$-Pflanzen, die $\mathrm{CO}_{2}$ über die nicht gegen ${ }^{13} \mathrm{CO}_{2}$ diskriminierende PEP-Carboxylase vorfixieren, bauen mehr schwere Kohlenstoffisotope in ihre Biomasse ein.

Wenn die stomatäre Leitfähigkeit hoch ist, bestimmt vor allem RubisCO das Maß der Diskriminierung. Ist dagegen die stomatäre Leitfähigkeit niedrig, wird der interne $\mathrm{CO}_{2}$-Pool mit ${ }^{13} \mathrm{CO}_{2}$ angereichert und RubisCO kann ${ }^{13} \mathrm{CO}_{2}$ nicht mehr so erfolgreich von der Fixierung ausschließen. Das bedeutet, dass der Kohlenstoff in der Biomasse ein ${ }^{13} \mathrm{C} /{ }^{12} \mathrm{C}$-Isotopenverhältnis annimmt, dass weniger negativ gegenüber der Atmosphäre ist als bei nicht eingeschränkter Leitfähigkeit.

Das ${ }^{13} \mathrm{C} /{ }^{12} \mathrm{C}$-lsotopenverhältnis wird als $\delta$-Wert ausgedrückt; $\delta^{13} \mathrm{C}$ wird als Relation zwischen der Probe und einem Standard bestimmt:

$$
\delta^{13} C=\left(\frac{\frac{{ }^{13} C}{{ }^{12} C} \text { in der Probe }}{\frac{{ }^{13} C}{{ }^{12} C} \text { im Standard }}-1\right) * 1000 \quad[\% 0]
$$

Als Standard dient $\mathrm{CO}_{2}$ aus PDB, einem fossilen Belemnit der Pee Dee Formation.

FARQUHAR et al. (1982) setzten die Diskriminierung von ${ }^{13} \mathrm{C}$ in Beziehung zum Verhältnis von internem zu externem $\mathrm{CO}_{2}$ Partialdruck $\left(\mathrm{p}_{\mathrm{i}} / \mathrm{p}_{\mathrm{a}}\right)$. Jedes pflanzliche Gewebe hat somit eine Isotopensignatur, die als über die Zeit gemittelter 
Schätzwert des Verhältnisses von $\mathrm{p}_{\mathrm{i}} / \mathrm{p}_{\mathrm{a}}$ angesehen werden kann (FARQUHAR \& RICHARDS 1984). Weil die Wassernutzungseffizienz (Assimilation/Transpiration) auch eine Funktion von $p_{i}$ ist, kann man das Isotopenverhältnis als ein Maß für die WUE ansehen (FARQUHAR et al. 1989).

Die Isotopen-Diskriminierung $\Delta$ wird im Bezug zum $\delta^{13} \mathrm{C}$-Wert der Luft angegeben. Pflanzen zeigen eine positive Diskriminierung $(\Delta)$ gegen ${ }^{13} \mathrm{C}$, die man nach LAMBERS et al. (1998) wie folgt berechnen kann:

$$
\Delta=\delta^{13} C_{\text {Luft }}-\delta^{13} C_{\text {Probe }}
$$

Das $\mathrm{CO}_{2}$ der Luft weist einen $\delta^{13} \mathrm{C}-$ Wert von $-8 \%$ gemessen gegen Pee Dee Belemnit auf (GRIFFITHS 1998).

1999 wurden bei Alhagi sparsifolia von jeweils 12 markierten Pflanzen 10 Blätter auf der AF- und ADN-Fläche geerntet. Da im Rahmen des Projektes auch $\delta^{15} \mathrm{~N}$ gemessen wurde, erfolgte die Ernte immer an denselben Pflanzen, um eventuelle Unterschiede zwischen $\mathrm{N}_{2}$ fixierenden und nicht fixierenden Pflanzen zu zeigen. Bei den anderen drei Arten wurden von jeweils sechs zufällig ausgewählten Pflanzen 10 Blätter geerntet, um eine Mischprobe über die Fläche zu erhalten. Die Probenahme für die Isotopen-Analysen erfolgte monatlich bei allen vier Pflanzenarten auf den $\mathrm{FN}$ - und DN-Flächen.

Von A. sparsifolia wurden auch im Jahr 2000 monatlich jeweils 10 Blätter von sechs Pflanzen pro Fläche geerntet, es wurden die FN-, FU-, DN- und DU-Flächen beprobt.

Die Proben wurden unmittelbar nach der Ernte für drei Minuten im Mikrowellenofen und anschließend bei $60^{\circ} \mathrm{C}$ bis zur Gewichtskonstanz im Trockenschrank getrocknet. Anschließend wurden die Proben in einer Schwingmühle (Retsch MM2, Wien, Österreich) gemahlen und in Zinnkapseln eingewogen.

Die Analyse erfolgte in Zusammenarbeit mit dem Institut für Chemische Pflanzenphysiologie, Universität Wien, in einem Isotopen-Massenspektrometer (IRMS, Deltaplus, Finnigan MAT, Bremen, Deutschland) mit vorgeschaltetem Elementaranalysator EA 1110 (CE Instruments) wie beschrieben bei ARNDT et al. (2000). 


\subsection{Statistik}

In den Abbildungen und Tabellen sind arithmetische Mittelwerte mit Standardfehlern dargestellt. Die Regressionsanalysen wurden mit dem Programm SigmaPlot 2000 (SPSS Inc., Chicago, Illinois, USA) berechnet, die Normalverteilung der Daten wurde geprüft. Alle anderen statistischen Analysen wurden mit SAS 8.01 (SAS Institute Inc., Cary, NC, USA) durchgeführt.

Die Daten wurden mit dem SHAPIRO-WILK-Test (SAS proc univariate) auf Normalverteilung geprüft. Bei einem Vergleich von Paardifferenzen wurden normalverteilte verbundene Stichproben mit dem T-Test für Paardifferenzen, nicht normalverteilte Stichproben mit dem U-Test (WILCOXON-Test) für abhängige Stichproben auf Signifikanz geprüft. Zweifache Varianzanalysen mit Messwiederholung in einer Vegetationsperiode wurden getrennt nach Arten für die Behandlungsvarianten "Nutzung" und „Bewässerung" für den Vergleich der Wasserhaushaltsparameter auf den genutzten und ungenutzten Flächen (2000) sowie auf den bewässerten und unbewässerten Flächen (1999) verwendet. Im Anschluss an eine ANOVA wurde der Mittelwerts-Test nach TUKEY oder nach SCHEFFÉ benutzt. 


\section{$4 \quad$ Ergebnisse}

\subsection{Klimatische Bedingungen}

Am Südrand der Taklamakan ist der Wind ein entscheidender Faktor, der über den Staub- und Sandtransport die Vegetation beeinflusst. Nach der Skala von BEAUFORT hebt bereits Wind der Stärke $4\left(5,5-7,9 \mathrm{~m}^{*} \mathrm{~s}^{-1}\right)$ Staub in die Luft. In Publikationen über die Taklamakan (z.B. XIA et al. 1993) werden, wenn es um Staubbelastung und Sandverwehungen geht, Windgeschwindigkeiten von über $5 \mathrm{~m}^{*} \mathrm{~s}^{-1}$ als sandbewegend angenommen. Solche Windgeschwindigkeiten treten in Qira häufig auf. In der Nacht vom 23. auf den 24. April 1999 wurden Windgeschwindigkeiten bis zu $10,98 \mathrm{~m}^{*} \mathrm{~s}^{-1}$ an der Klimastation Qira_1 und 8,04 $\mathrm{m}^{*} \mathrm{~s}^{-1}$ an der Klimastation Qira_2 gemessen. Es wurde so viel Staub und Sand aufgewirbelt, dass es zu einem jähen Temperatursturz kam und die Strahlungssummen in den darauf folgenden Tagen nur noch halb so hohe Werte erreichten wie vor dem Sturm (siehe auch Tab. 4). Solche Starkwindereignisse traten mehrfach im Untersuchungszeitraum auf und werden in ihren Auswirkungen auf die untersuchten Pflanzen später beschrieben.

Die Windgeschwindigkeiten an der Klimastation Qira_1 (auf der Alhagi-Fläche) sind in der Regel höher als an der Station Qira_2 (auf der Tamarix-Fläche), da im Vorland westlich der Alhagi-Fläche die Dünen niedriger und die Vegetation offener ist (BRUELHEIDE et al. 2003). In Bezug auf die anderen gemessenen Parameter unterscheiden sich die Klimastationen kaum. Daher wurden für vergleichende Darstellungen von Wasserhaushaltsdaten und Witterung auch die Werte der jeweils anderen Station herangezogen, wenn die den Untersuchungsflächen am nächsten gelegene Station aufgrund technischer Probleme keine Werte lieferte.

Anhand der monatlichen Strahlungssummen und Monatsmitteltemperaturen kann man die beiden Untersuchungsjahre vergleichen (Abb. 4.1.1). Im Jahr 2000 waren die Monate Mai und August strahlungsreicher, 1999 dagegen der Juli und die Herbstmonate. Unterschiede in der Monatsmitteltemperatur finden sich vor allem im Mai (2000 wärmer) und September (1999 wärmer). 


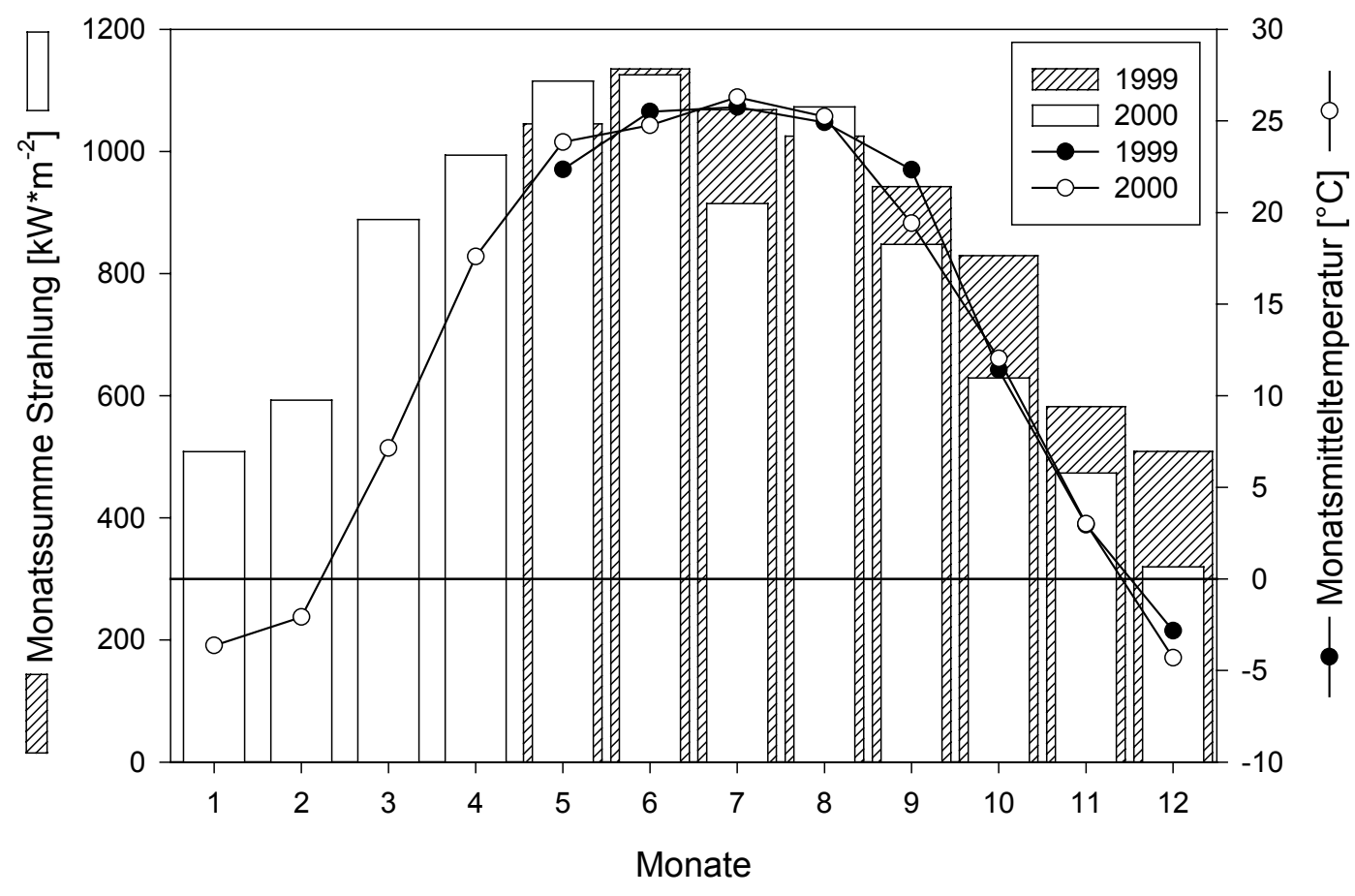

Abb. 4.1.1: Monatliche Strahlungssummen der Globalstrahlung und Monatsmitteltemperaturen 1999 und 2000.

Die Messwerte sind zusammengefasst von beiden Wetterstationen Qira_1 (AFN-Fläche) und Qira_2 (TFN-Fläche).

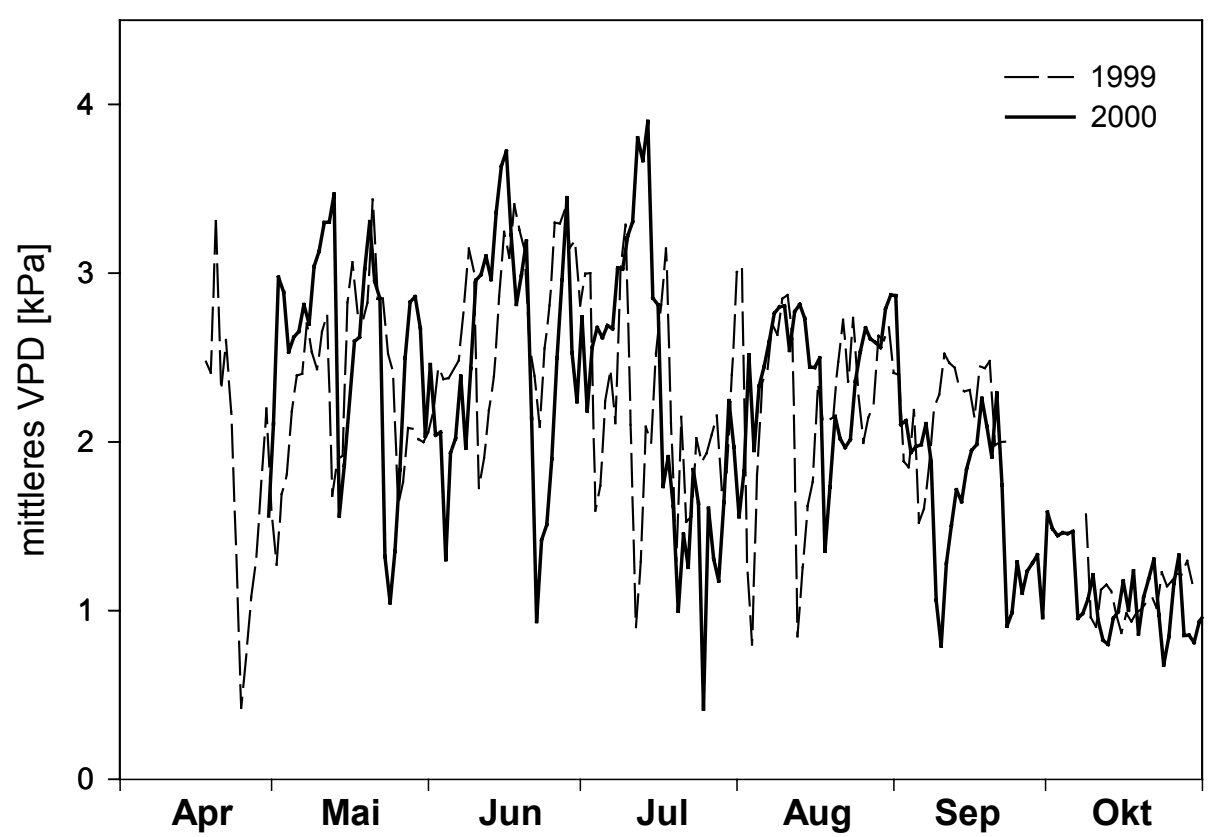

Abb. 4.1.2: Tagesmittelwerte des Wassersättigungsdefizits (VPD) während der Vegetationsperioden der Untersuchungsjahre 1999 und 2000.

Die Mittelwerte wurden berechnet aus Messwerten der Wetterstationen Qira_1 und Qira_2. 
Das Wasserdampfsättigungsdefizit (VPD) erreichte an sonnigen Tagen bis zu $7 \mathrm{kPa}$ am Nachmittag und sank häufig auch nachts nicht unter $1 \mathrm{kPa}$ (nicht dargestellt). In Abb. 4.1.2 sind die Tagesmittelwerte des VPD gezeigt. Das mittlere VPD erreicht vom Frühjahr bis zum Spätsommer hohe Werte, erst Ende September sinkt es rapide ab. Man kann die Tage mit hohen Windgeschwindigkeiten an einem plötzlichen Einbruch des VPD aufgrund von Staubaufwirbelung erkennen (z.B. Ende April und Mitte Juli 1999, Ende Mai, Mitte Juni und Ende Juli 2000).

\subsection{Bodenwassergehalte}

1999 lagen die Bodenwassergehalte auf allen Flächen vor der experimentellen Überflutung unter 5 Vol. \% (Abb. 4.2.1), außer an den vom Grundwasser beeinflussten Sonden auf der Tamarix- $(650-660 \mathrm{~cm})$ und Populus-Fläche (340-350 und 456-466 cm).

Die Überflutung zeigte auf allen Untersuchungsflächen außer der Tamarix-Fläche einen deutlich messbaren Effekt:

Auf der Alhagi-Fläche stieg der Wassergehalt des Oberbodens (20-30 cm) sprunghaft von 1 auf 23 Vol. \% an, mit einer Zeitverschiebung von zwei Tagen konnte man den Anstieg in 90-100 cm Tiefe verfolgen. Nach ungefähr 5-6 Wochen war das Wasser in eine Tiefe von $2 \mathrm{~m}$ vorgedrungen. In der Tiefe von 340-350 cm und tiefer war keine Veränderung des Bodenwassergehaltes feststellbar.

Im Oberboden der Populus-Fläche stieg der Wassergehalt von 3 auf 25 Vol. \% in der Tiefe von 20-30 cm. Mit einer Zeitverzögerung von drei Wochen war auch ein Anstieg in $1 \mathrm{~m}$ Tiefe zu verzeichnen. In $2 \mathrm{~m}$ Tiefe war keine Veränderung mess-bar (Abb. 4.2.1).

Auf der Freifläche (open ground) stiegen die Wassergehalte in 20-30 cm und 90$100 \mathrm{~cm}$ Tiefe stiegen zeitgleich von 2 auf 23 bzw. 22 Vol. \% an, der Oberboden trocknete etwas schneller wieder aus und hatte schon nach zwei Tagen einen Wert von 12 Vol. \% erreicht, während in $1 \mathrm{~m}$ Tiefe noch $17 \mathrm{Vol}$ \% zu messen waren. Mit einer Zeitverzögerung von zehn Tagen stieg auch der Wassergehalt in $2 \mathrm{~m}$ Tiefe an. 


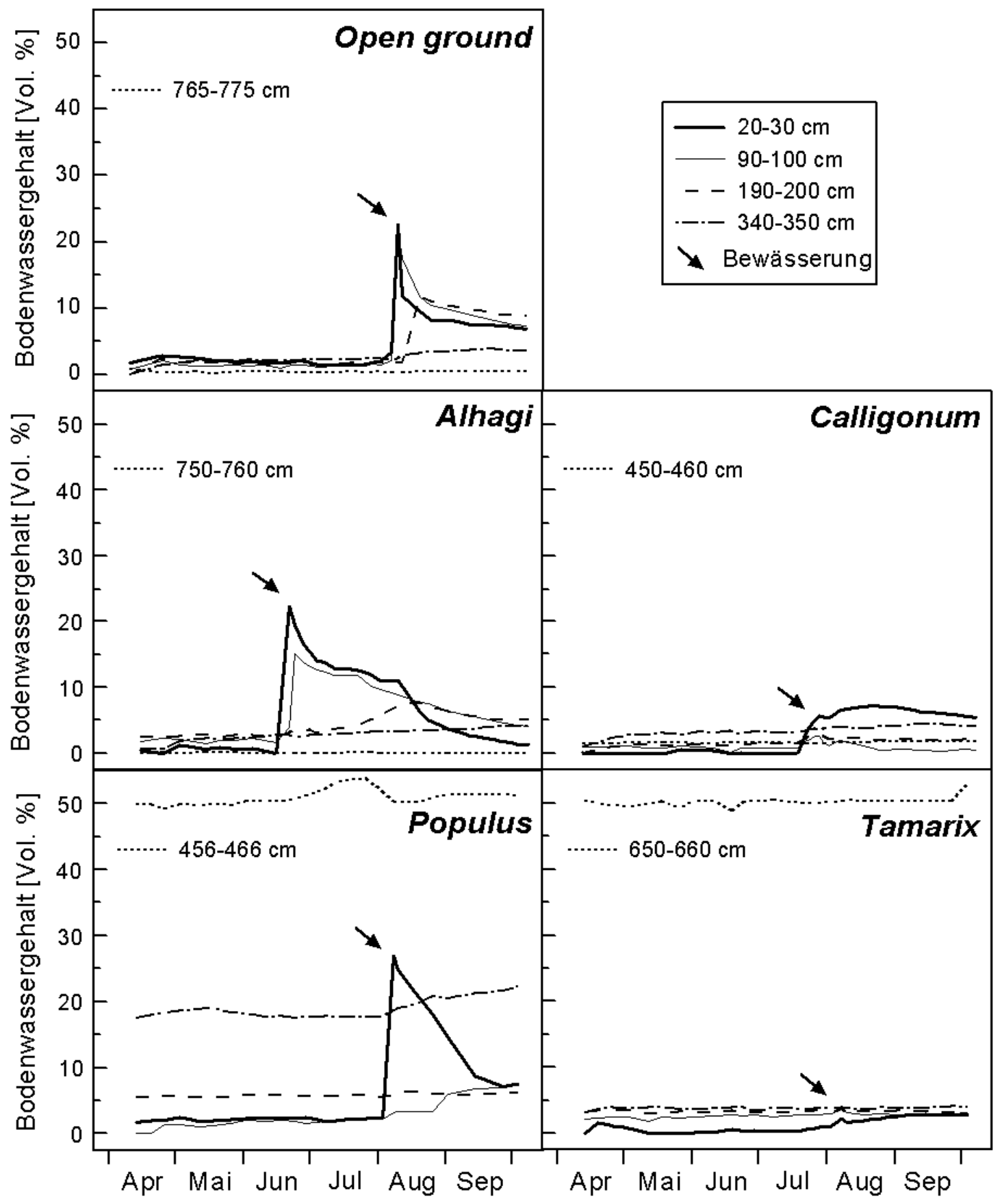

Abb. 4.2.1: Bodenwassergehalte (Vol. \%) auf den Untersuchungsflächen 1999.

Die Tiefe der oberen vier TDR-Sonden ist auf allen Flächen gleich (20-30 $\mathrm{cm}, 90-100 \mathrm{~cm}, 190-200 \mathrm{~cm}, 340-350 \mathrm{~cm}$ ), für die tiefste Sonde ist die jeweilige Bodentiefe in der Grafik angegeben. Pfeile kennzeichnen den Zeitpunkt der im Sommer 1999 durchgeführten experimentellen Bewässerung. 
Auf der Calligonum-Fläche stieg der Wassergehalt des Oberbodens nur auf etwa 7 Vol \% an (Abb. 4.2.1). Schon ab $1 \mathrm{~m}$ Tiefe war kein Anstieg des Bodenwassergehaltes mehr $\mathrm{zu}$ messen. Von dem geringen Anstieg des Bodenwassergehaltes in den oberen Bodenschichten und der deutlichen Umgestaltung der Fläche durch Abflussrinnen konnte man auf eine eher kurze Verweildauer des Wassers auf der Fläche schließen. Es muss davon ausgegangen werden, dass das Überflutungswasser schnell über die Fläche strömte und den Boden kaum infiltrieren konnte.

Durch das Einbringen der TDR Sonden auf einer flachen Düne (zwischen der Vegetation) ist es bei der Bewässerung auf der Tamarix-Fläche zu keinem messbaren Anstieg des Bodenwassergehaltes gekommen. Das Überflutungswasser reichte nicht bis auf die Düne, sondern stand in etwa einem Meter Entfernung von den TDR-Sonden an (Höhenunterschied höchstens $50 \mathrm{~cm}$ ). Es lässt sich daraus schließen, dass kein oder nur ein geringer lateraler Wassertransport in den vorliegenden Schluffböden auftritt.

Am Ende der Messperiode 1999 (Anfang Oktober) lagen die Bodenwassergehalte des Oberbodens noch 0,7 - 2 Vol. \% (A. sparsifolia, T. ramosissima) bzw. 5 Vol. \% (C. caput-medusae, $P$. euphratica, Open ground) über dem Wert vor der Überflutung (Abb. 4.2.1).

Im Jahr 2000 wurden mit 0 bis 7 Vol. \% wieder ähnliche Bodenwassergehalte wie Anfang 1999 gemessen, die Ausnahmen bildeten wie im Vorjahr die grundwassernahen Sonden auf der Tamarix- und Populus-Fläche. Im Jahresverlauf änderten sich die Wassergehalte auf keiner der Flächen (Abb. 4.2.2). 


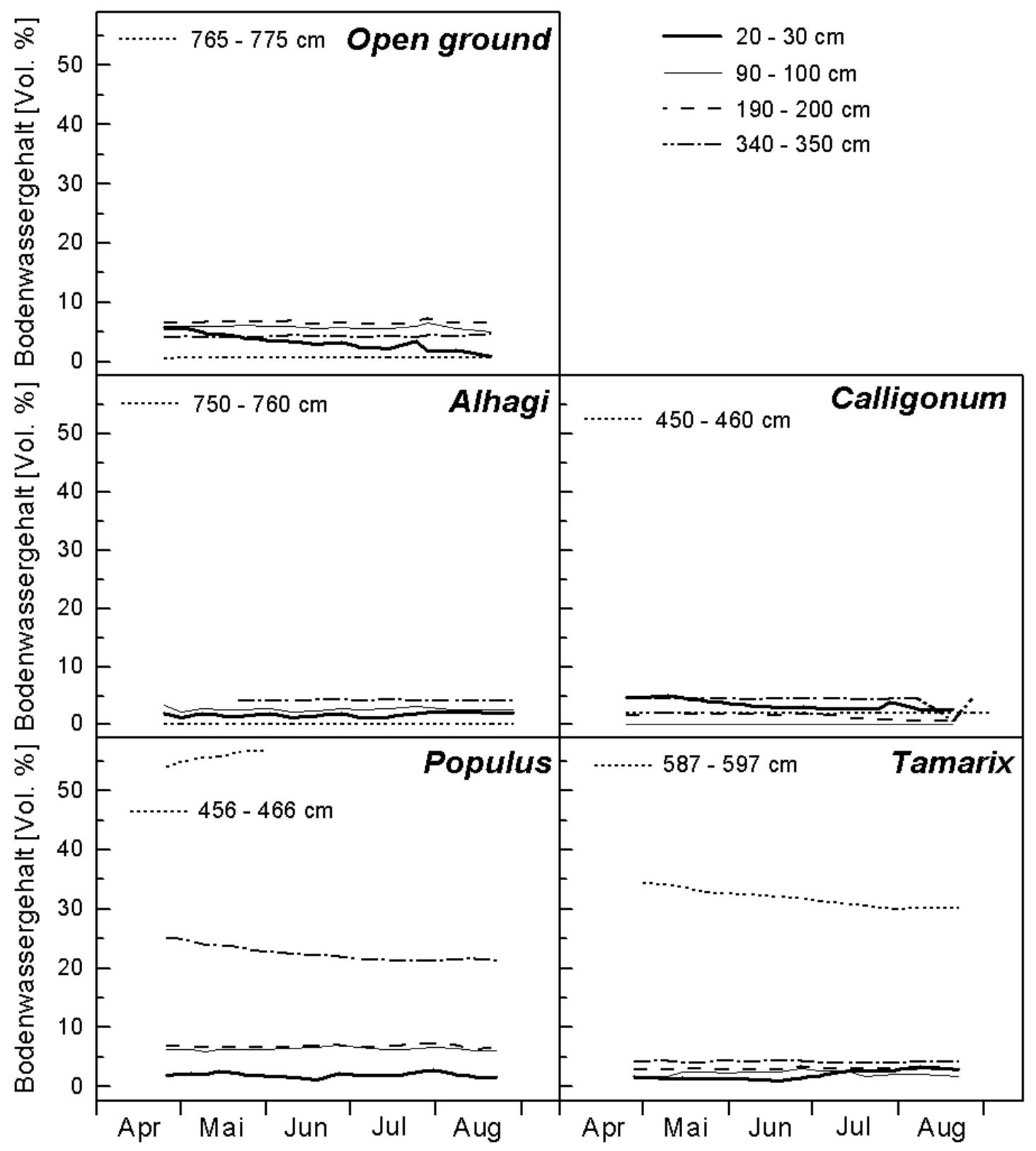

Abb. 4.2.2: Bodenwassergehalte (Vol. \%) auf den Untersuchungsflächen 2000.

Die Tiefe der oberen vier TDR-Sonden ist auf allen Flächen gleich (20-30 $\mathrm{cm}, 90-100 \mathrm{~cm}, 190-200 \mathrm{~cm}, 340-350 \mathrm{~cm}$ ), für die tiefste Sonde ist die jeweilige Bodentiefe in der Grafik angegeben. 


\subsection{Entwicklung von Blattbiomasse und Blattflächenindex (LAI)}

Die Entwicklung der transpirierenden Biomasse wurde in beiden Untersuchungsjahren durch monatliche Ernten bestimmt. Auf Grundlage dieser Ernten wurden Tagesblattmassen ermittelt, die für die Berechnung der blattgewichtsbezogenen Saftllussraten benötigt wurden.

A. sparsifolia schloss 1999 nach einer Phase exponentiellen Wachstums den jährlichen Zuwachs schon Anfang Juni ab (Abb. 4.3.1). Die Blattmasse änderte sich danach nicht mehr signifikant. Bei den Ernten im folgenden Jahr stieg jedoch das Trockengewicht der Büsche bis Anfang September an, doch war ab Juli keine signifikante Zunahme mehr gegeben (Abb. 4.3.1). Auch C. caput-medusae erreichte den maximalen Biomassewert schon Mitte Juni 1999, im Jahr 2000 war der Anstieg etwas flacher. 2000 wurde die maximale Biomasse ein wenig später im Jahr erreicht als 1999. Dies kann vermutlich auf eine geringere Strahlungssumme im Juli 2000 verglichen mit dem Vorjahr zurückgeführt werden (Abb. 4.1.1). Bei $T$. ramosissima stieg Masse der transpirierenden Organe zunächst steil an und erreichte im Juni Biomassewerte, von dem sich spätere Werte nicht mehr signifikant unterschieden.

Bei $P$. euphratica wurde auf der PF-Fläche mit etwa $2,66 \mathrm{~m}^{2 *} \mathrm{~m}^{-2}$ der maximale Blattflächenindex (LAI) Ende Juli 1999 erreicht (Abb. 4.3.1), aber auch hier ist die Differenz zu den im Juni erreichten Werten nicht mehr signifikant. Die PD-Fläche wies mit etwa $2,28 \mathrm{~m}^{2 *} \mathrm{~m}^{-2}$ einen deutlich niedrigeren LAI auf. Im Jahr 2000 wurden auf allen Flächen etwas höhere Werte berechnet. Im Juni und Juli 2000 konnte für die PFN-Fläche ein LAl von etwa $3 \mathrm{~m}^{2 *} \mathrm{~m}^{-2}$ ermittelt werden, der im Spätsommer, vermutlich durch die Auswirkung des Blattfraßes durch Raupen, wieder etwas abfiel. Die PFU-Fläche erreichte nur einen LAI von gut $1,91 \mathrm{~m}^{2 *} \mathrm{~m}^{-2}$. Auf der PDFläche wurde wie schon 1999 auch 2000 ein geringerer LAI als auf der PF-Fläche festgestellt, es wurde auf der PDN-Fläche ein LAI von $2,52 \mathrm{~m}^{2 *} \mathrm{~m}^{-2}$ und auf der PDU-Fläche ein Wert von $1,81 \mathrm{~m}^{2 *} \mathrm{~m}^{-2}$ erreicht.

Anhand der aus diesen Ernten ermittelten Kurven wurde mit Hilfe der täglichen Biomassenwerte der trockengewichtsbezogene Saftluss [g $\mathrm{H}_{2} \mathrm{O}^{*} \mathrm{TG}^{-1 *} \mathrm{Tag}^{-1}$ ] berechnet. 

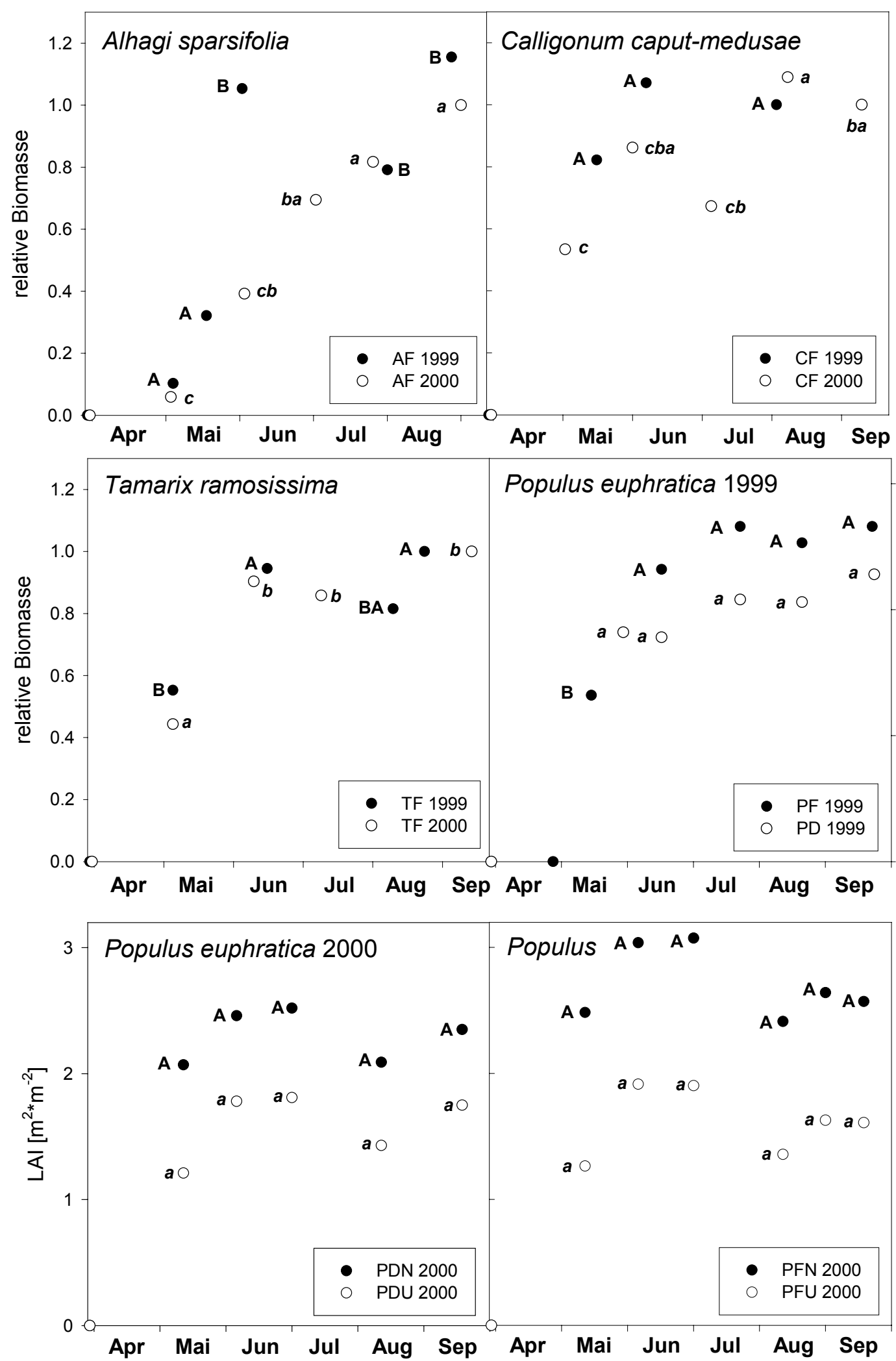

Abb. 4.3.1: Entwicklung der Blattbiomasse bzw. des Blattflächenindexes (LAl) der untersuchten Arten in der Vegetationsperiode 1999 und 2000.

Die Entwicklung der transpirierenden Biomasse wurde für $A$. sparsifolia, $C$. caput-medusae und $T$. ramosissima durch monatliche Ernten ermittelt. Der LAl von $P$. euphratica wurde aus Messungen der relativen Beleuchtungsstärke im Bestand und einer Ernte im Sommer 1999 für die verschiedenen Teilflächen berechnet. 


\subsection{Wasserpotentiale}

1999 wurde auf den FN-Flächen immer an den Tagen ein Tagesgang des Blattwasserpotentials gemessen, an denen auch Transpirationsmessungen stattfanden. Graphisch dargestellt sind die Mittelwerte der Untersuchungsflächen (Abb. 4.4.1 bis 4.4.4). Im folgenden werden Mittelwerte pro Pflanze genannt.

Bei Alhagi sparsifolia wurden die niedrigsten predawn-Werte $\left(\Psi_{\mathrm{pd}}\right)$ des Jahres im September erreicht; insgesamt zeigte $A$. sparsifolia die höchsten predawn-Wasserpotentiale der untersuchten Arten (Abb. 4.4.1). Selbst bei einem sehr hohem VPD von 5,5 kPa wurden am 09.07.1999 nur Mittagswasserpotentiale ( $\left.\Psi_{\mathrm{md}}\right)$ von $1,86 \mathrm{MPa}$ als Mittelwert der Fläche erreicht, ähnlich wie an Tagen mit geringerem VPD. Die Mittagswasserpotentiale sanken im Lauf der Vegetationsperiode kontinuierlich ab und erreichten im September den niedrigsten gemessenen Wert $(-2,36 \mathrm{MPa})$.

Ein Vergleich der Wasserpotentiale vor und nach der Bewässerung ist kritisch, da die letzte Messung mehr als einen Monat vor der Bewässerung stattfand und die jahreszeitliche Veränderung einen möglichen Bewässerungseffekt überlagern kann.

Calligonum caput-medusae zeigte im Lauf der Vegetationsperiode nur eine geringe Variabilität der predawn-Wasserpotentiale $\left(\Psi_{\mathrm{pd}}\right)$ von $-0,68$ (Juni) bis zu $-0,84 \mathrm{MPa}$ im August. Die Mittagsblattwasserpotentiale schwankten zwischen -1,76 MPa im Mai bis zu -1,95 MPa im September, damit zeigte C. caput-medusae die höchsten $\Psi_{\mathrm{md}}$ im Artvergleich (Abb. 4.4.2). Die Wasserpotentiale zeigten kaum eine Veränderung durch die Bewässerung, das Mittagswasserpotential war nach der Bewässerung geringfügig höher (nicht signifikant, Abb. 4.4.2).

Die niedrigsten Wasserpotentiale der untersuchten Arten wies $T$. ramosissima auf (Abb. 4.4.3). Die predawn-Wasserpotentiale sanken im Lauf der Vegetationsperiode auf -1,22 MPa im Juli. In den anderen Monaten lagen sie nur zwischen $-0,89 \mathrm{MPa}$ (Mai) und -1,18 MPa (August). Schon im Mai wurden Mittagspotentiale von -2,38 MPa an einem Tag mit sehr hohem VPD erreicht. Die Messung im Juni (11.06.1999) musste wegen eines Sandsturms abgebrochen werden. Obwohl das VPD nur Werte von maximal 1,5 kPa erreichte, wurden trotzdem Wasserpotentiale von $-2,39 \mathrm{MPa}$ gemessen. Die niedrigsten Mittagswasserpotentiale wurden mit $-3,26 \mathrm{MPa}$ im August erreicht. Die $\Psi_{\mathrm{md}}$ waren nach der Überflutung niedriger als 
vorher, was aber durch das viel höhere VPD des Messtages zu erklären ist (Abb. 4.4.3), die $\Psi_{\text {pd }}$ höher.

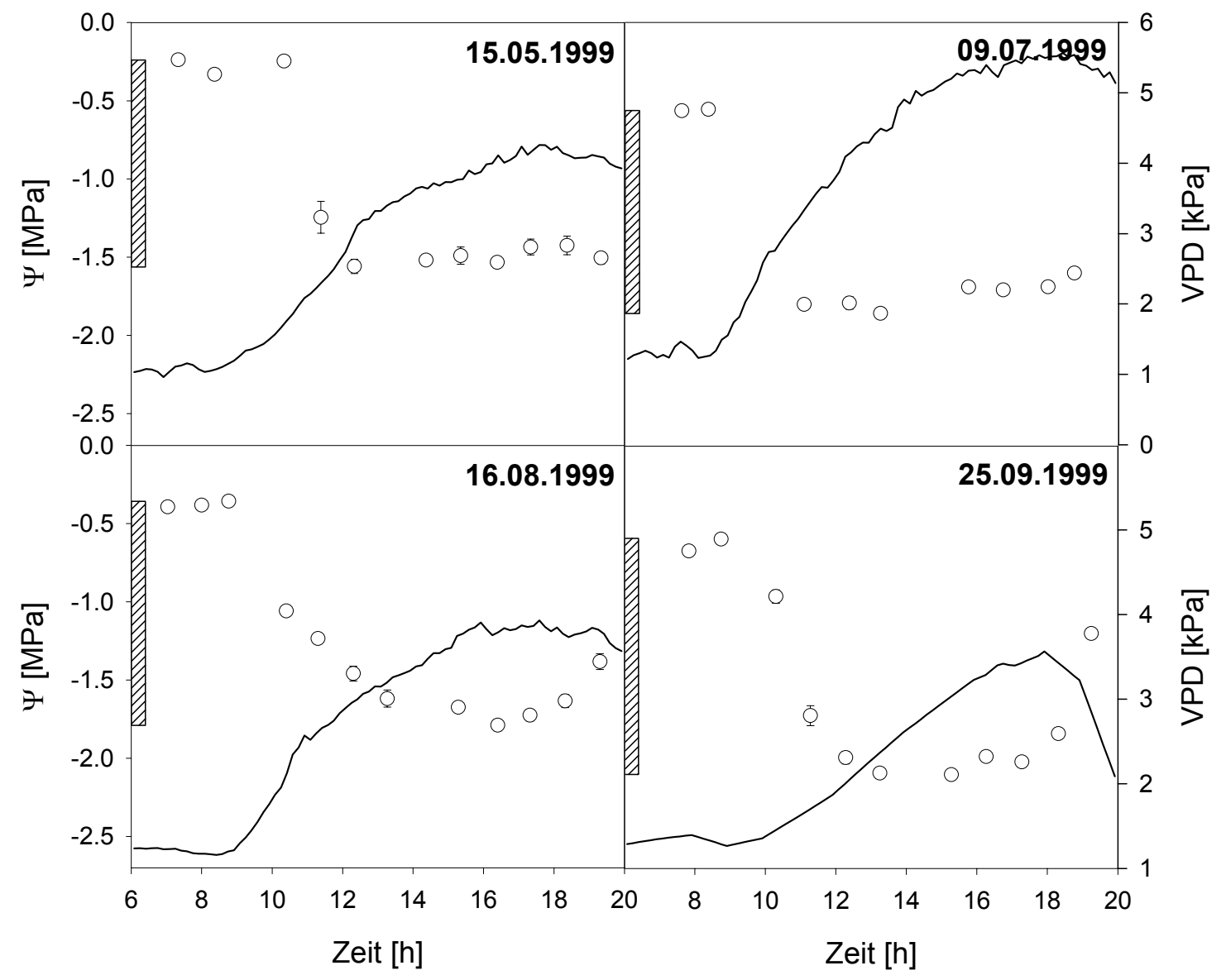

Abb. 4.4.1: Tagesgänge des Blattwasserpotentials und des Wasserdampfsättigungsdefizits (VPD) von Alhagi sparsifolia 1999.

Gemessen wurde auf der bewässerten ungenutzten Fläche (AFN). Dargestellt sind die Mittelwerte des Wasserpotentials aus 18 Messungen (Kreise). Als schraffierter Balken ist die maximale Differenz des Wasserpotentials der Messtage gekennzeichnet. Das VPD (Linie) wurde aus Daten der Wetterstation auf der Fläche berechnet, nur am 25.09. wurden Werte der staatlichen Wetterstation in Qira verwendet. 


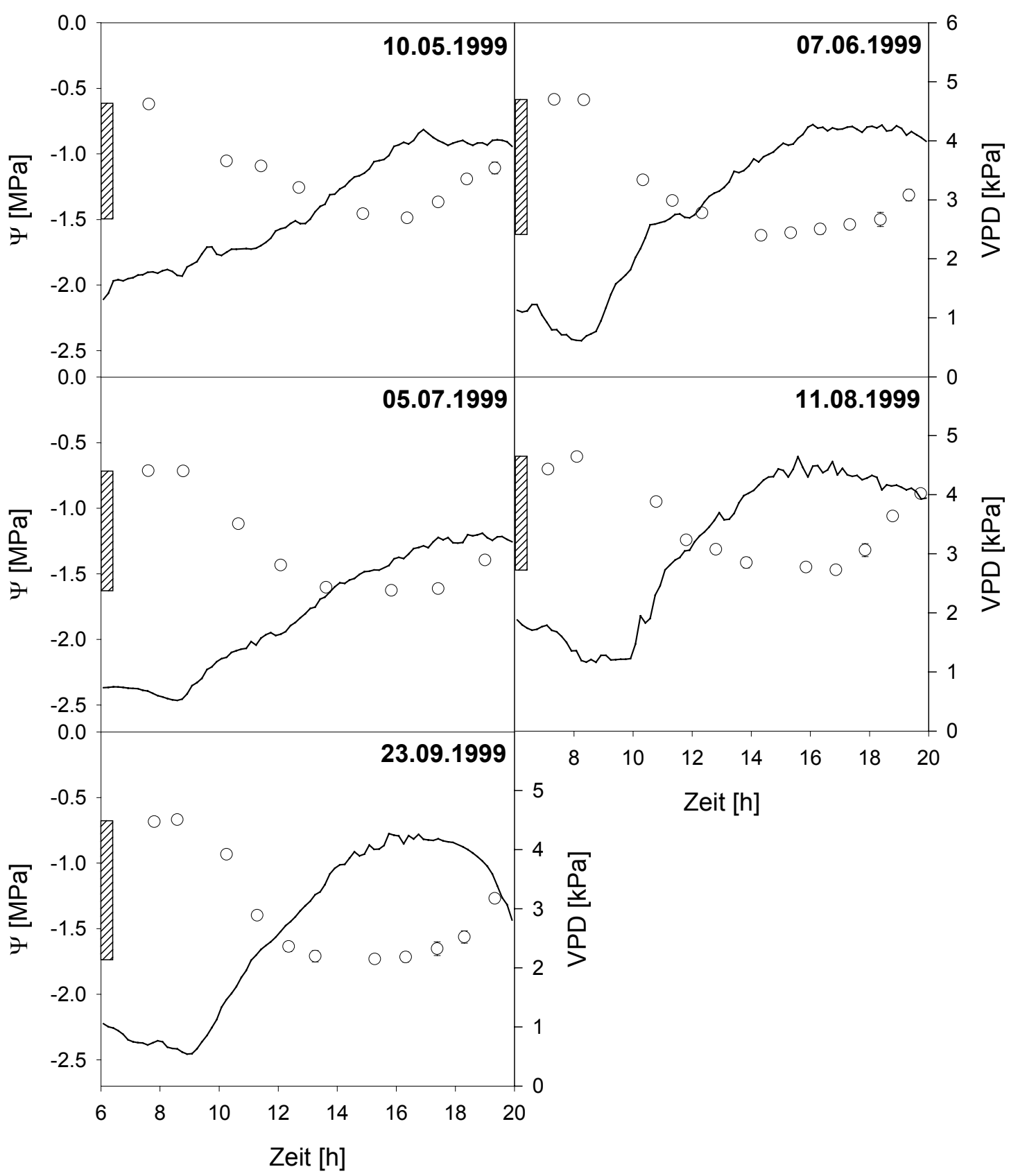

Abb. 4.4.2: Tagesgänge des Blattwasserpotentials und des Wasserdampfsättigungsdefizits (VPD) von Calligonum caput-medusae 1999.

Gemessen wurde auf der bewässerten ungenutzten Fläche (CFN). Dargestellt sind die Mittelwerte des Wasserpotentials aus 18 Messungen (Kreise). Als schraffierter Balken ist die maximale Differenz des Wasserpotentials der Messtage gekennzeichnet. Das VPD (Linie) wurde aus Daten der Wetterstation auf der AFN-Fläche berechnet. 


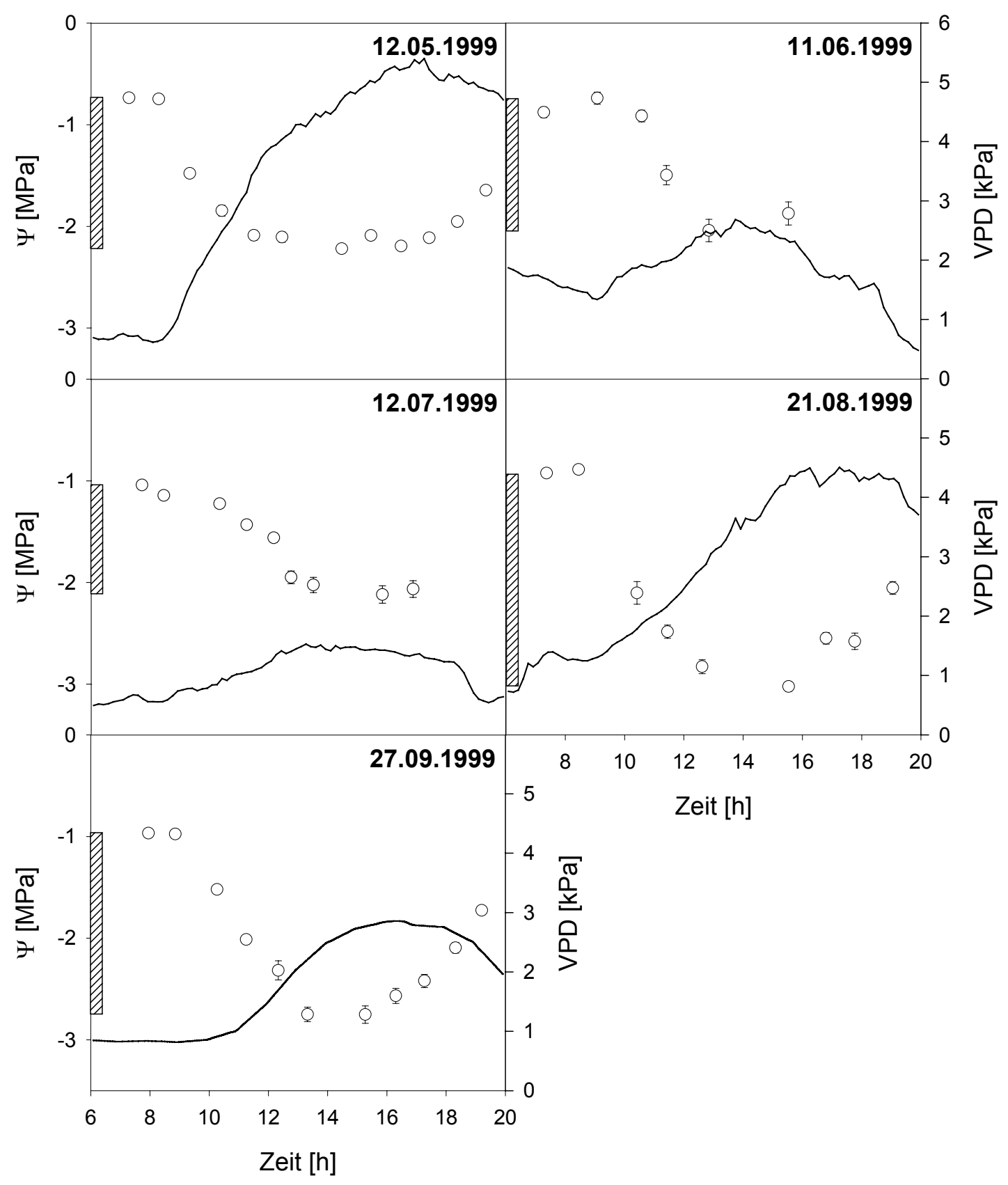

Abb. 4.4.3: Tagesgänge des Blattwasserpotentials und des Wasserdampfsättigungsdefizits (VPD) von Tamarix ramosissima 1999.

Gemessen wurde auf der bewässerten ungenutzten Fläche (TFN). Dargestellt sind die Mittelwerte des Wasserpotentials aus 18 Messungen (Kreise). Als schraffierter Balken ist die maximale Differenz des Wasserpotentials der Messtage gekennzeichnet. Das VPD (Linie) wurde aus Daten der Wetterstation auf der Fläche berechnet, nur am 27.09. wurden Werte der staatlichen Wetterstation in Qira verwendet. 


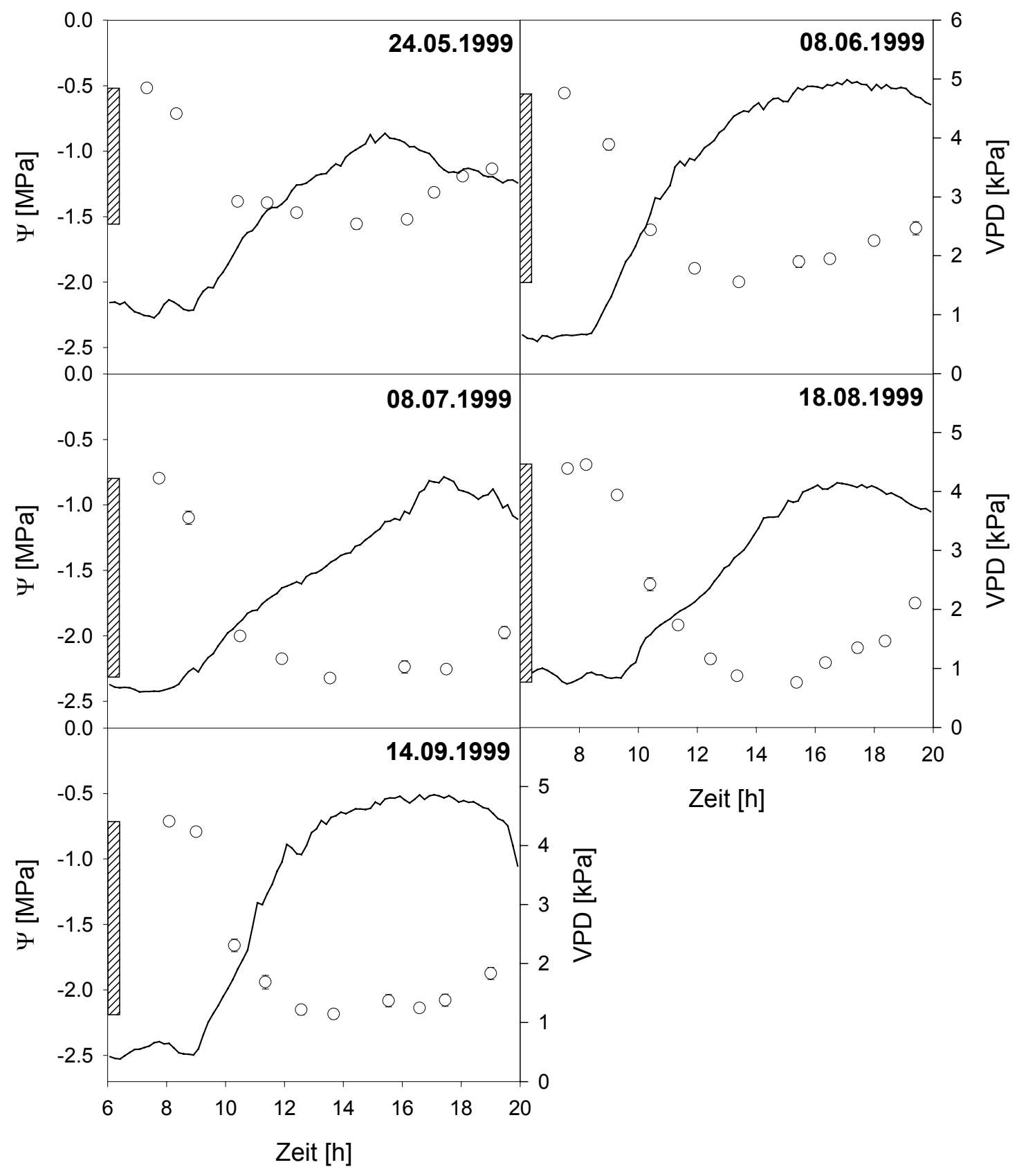

Abb. 4.4.4: Tagesgänge des Blattwasserpotentials und des Wasserdampfsättigungsdefizits (VPD) von Populus euphratica 1999.

Gemessen wurde auf der bewässerten ungenutzten Fläche (PFN). Dargestellt sind die Mittelwerte des Wasserpotentials aus 18 Messungen (Kreise). Als schraffierter Balken ist die maximale Differenz des Wasserpotentials der Messtage gekennzeichnet. Das VPD (Linie) wurde aus Daten der Wetterstation auf der TFN-Fläche berechnet. 
Populus euphratica zeigte einen ähnlichen Jahresverlauf wie die anderen Arten mit den geringsten Werten im Sommer (Abb. 4.4.4). Die predawn-Wasserpotentiale waren im Mai mit -0,52 MPa noch relativ hoch (Flächenmittelwert). Im Juli wurden mit -0,9 MPa die niedrigsten $\Psi_{\text {pd }}$ des Jahres erreicht. Die Mittagswasser-potentiale sanken von $-1,72 \mathrm{MPa}$ im Mai auf bis zu -2,51 MPa im Sommer. Die größte Spanne zwischen predawn- und Mittagswerten wurde im August gemessen. Die niedrigsten Wasserpotentiale wurden im Juli und August gemessen, die Messtage mit dem höchsten VPD aber waren am 08.06.1999 und 14.09.1999. Die Mittagswasserpotentiale änderten sich nach der Bewässerung nicht, die predawnPotentiale waren etwas höher, unterschieden sich aber auch nicht signifikant von den Werten vor der Bewässerung (Abb. 4.4.5).

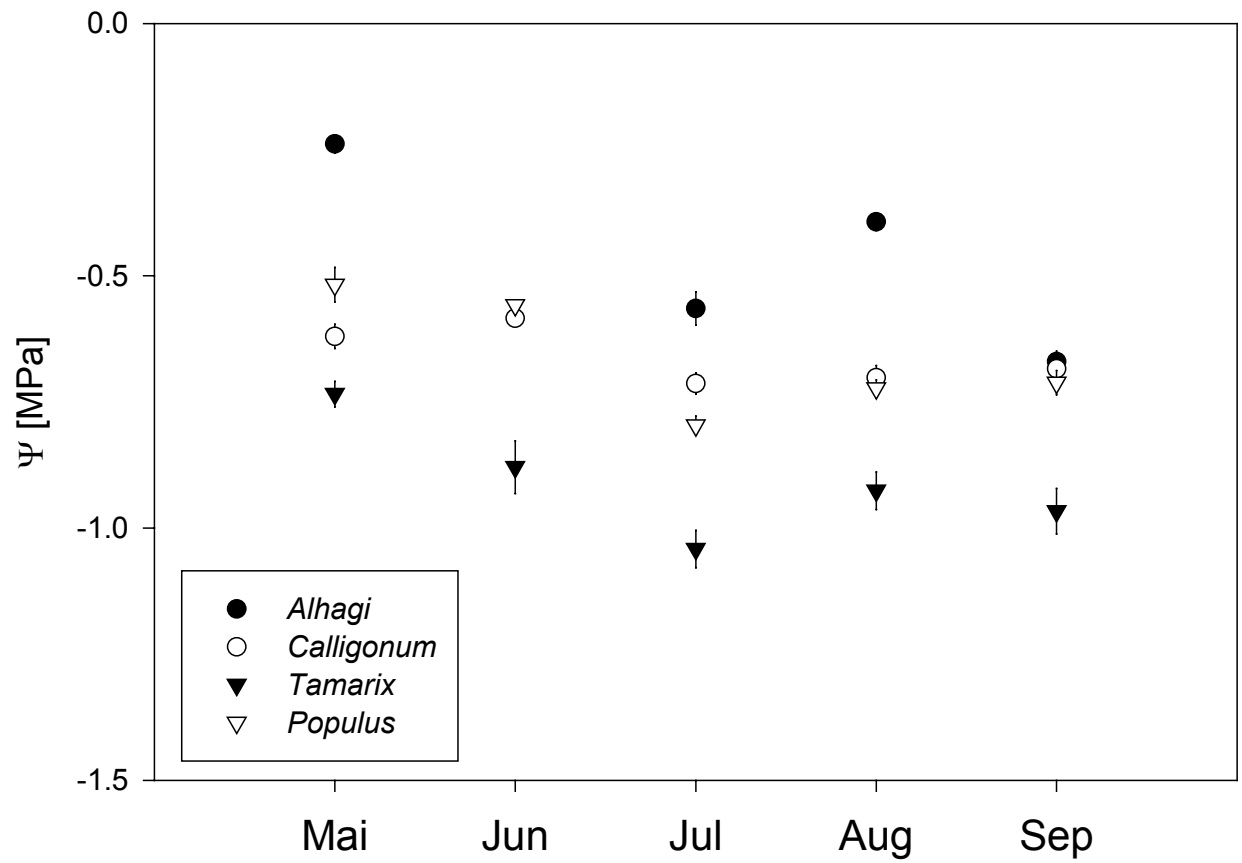

Abb. 4.4.5: Predawn-Wasserpotentiale der untersuchten Arten 1999.

Dargestellt sind die auf den bewässerten ungenutzten Flächen (FN) gemessenen Wasserpotentiale [MPa] als Flächenmittelwerte aus 18 Messungen.

Zusammenfassend kann man sagen, dass bei allen vier Arten die predawnWasserpotentiale wenig negativ im Vergleich mit anderen Arten in Trockengebieten waren. Die niedrigsten Werte im Artvergleich erreichte an allen Messterminen $T$. ramosissima, gefolgt von $P$. euphratica und $C$. caput-medusae. 
A. sparsifolia hatte die höchsten $\Psi_{\text {pd }}$ der untersuchten Arten (Abb. 4.4.5).

Bei den Mittagswasserpotentialen blieb die Reihenfolge fast erhalten, nur wurden bei C. caput-medusae die höchsten Werte gemessen $(T$. ramosissima $<P$. euphratica < A. sparsifolia < C. caput-medusae).

Betrachtet man die größten Differenzen des Wasserpotentials im Tagesverlauf (Tab. 4), ergibt sich im Mai, August und September die größte Spanne zwischen predawn- und midday-Potentialen bei $T$. ramosissima, im Juni und Juli aber bei $P$. euphratica. C. caput-medusae hat während der gesamten Vegetationsperiode die geringste Spanne.

Tab. 4: Maximale Differenz im Wasserpotential 1999.

Differenzen zwischen predawn- und midday-Wasserpotential [MPa] bei den untersuchten Arten während der Messperiode 1999.

\begin{tabular}{cccccc}
\hline $\mathbf{1 9 9 9}$ & Mai & Juni & Juli & August & September \\
\hline A. sparsifolia & 1,29 & - & 1,30 & 1,43 & 1,51 \\
C. caput-medusae & 0,87 & 1,04 & 0,91 & 0.86 & 1,06 \\
T. ramosissima & 1,48 & 1,30 & 1,08 & 2,13 & 1,79 \\
P. euphratica & 1,04 & 1,44 & 1,53 & 1,66 & 1,47 \\
\hline
\end{tabular}

Im Untersuchungsjahr 2000 wurden keine Tagesgänge des Wasserpotentials, sondern nur predawn- und Mittagswasserpotentiale gemessen. Die Messungen erfolgten parallel auf den ungenutzten (FN) und genutzten (FU) Flächen (Abb. 4.4.6 und 4.4.7). Nur bei Alhagi sparsifolia wurde ausschließlich auf der ungenutzten Fläche gemessen.

A. sparsifolia zeigte wie schon 1999 sehr wenig negative predawn-Wasserpotentiale, die geringsten $\Psi_{\mathrm{md}}$ wurden im September gemessen (Abb. 4.4.6). Bei C. caput-medusae änderten sich die Wasserpotentiale während der Vegetationsperiode nicht (Abb. 4.4.6). Zwischen ungenutzter und genutzter Fläche traten keine signifikanten Unterschiede auf. 


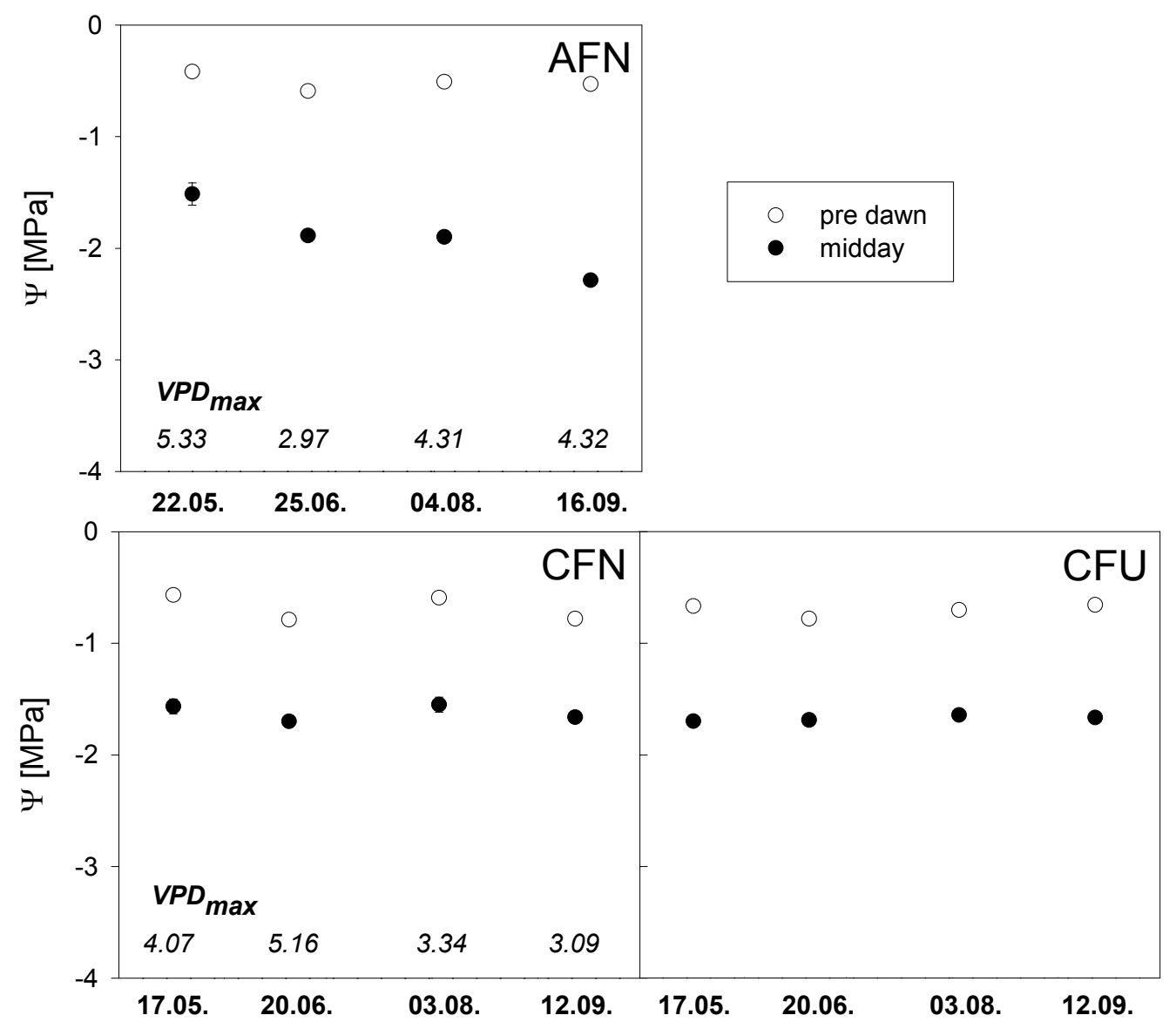

Abb. 4.4.6: Predawn- und midday-Wasserpotentiale von Alhagi sparsifolia und Calligonum caput-medusae 2000.

Dargestellt sind die parallel gemessenen Wasserpotentiale [MPa] auf den im Vorjahr bewässerten ungenutzten (FN) und genutzten (FU) Flächen als Mittelwerte aus 12 Messungen. Das maximale VPD an den Messtagen ist angegeben.

Wie schon 1999 erreichte T. ramosissima auch 2000 sowohl predawn als auch mittags die negativsten Wasserpotentiale im Artvergleich (Abb. 4.4.7). Es konnten wegen einer Käferkalamität, bei der alle Tamarisken auf der Untersuchungsfläche, ja sogar im gesamten westlichen Oasenvorland kahlgefressen wurden, im Sommer keine Transpirationsdaten erhoben werden. Daher wurden auch keine Wasserpotentiale gemessen. Die $\Psi_{\mathrm{pd}}$ und $\Psi_{\mathrm{md}}$ auf der Tamarix-Fläche erreichten 2000 ähnliche Werte wie 1999, die nicht genutzte und genutzte Fläche unterschieden sich an keinem der beiden Termine signifikant. $P$. euphratica wies an keinem Termin predawn-Wasserpotentiale unter -1 MPa auf, die Mittagspotentiale lagen ähnlich wie 1999 und waren am negativsten im August (Abb. 4.4.7). Die $\Psi_{\mathrm{md}}$ 
waren an drei Terminen (Mai, August, September) auf der genutzten Fläche signifikant höher und unterschieden sich nur Anfang Juli nicht.

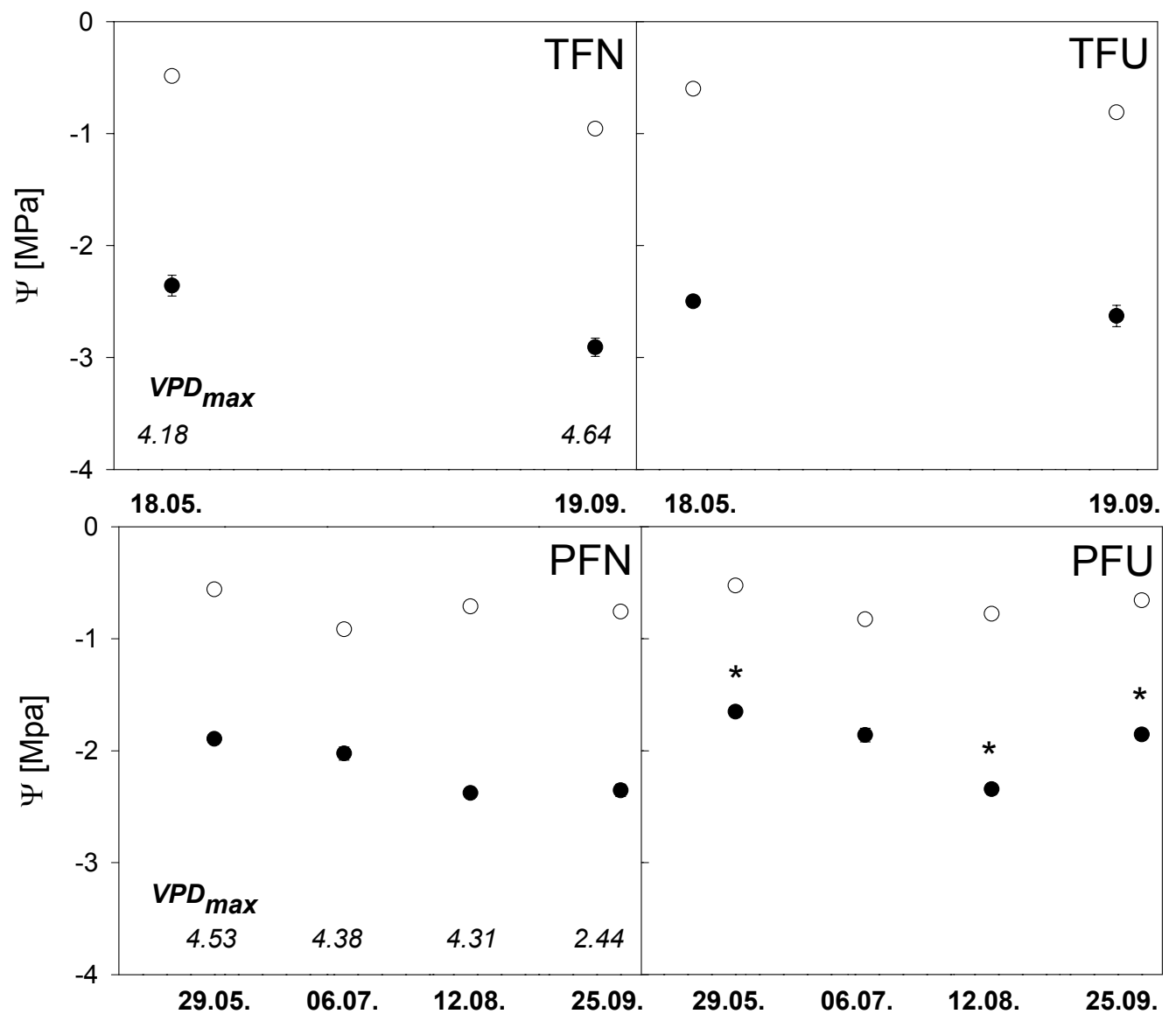

Abb. 4.4.7: Predawn- und midday-Wasserpotentiale von Tamarix ramosissima und Populus euphratica 2000.

Dargestellt sind die parallel gemessenen Wasserpotentiale [MPa] auf den im Vorjahr bewässerten ungenutzten (FN) und genutzten (FU) Flächen als Mittelwerte aus 12 Messungen. Das maximale VPD an den Messtagen ist angegeben. Sternchen $\left(^{*}\right)$ kennzeichnen einen signifikanten Unterschied zwischen $\mathrm{N}$ - und U-Flächen am gleichen Messtermin. 


\subsection{Transpiration und stomatäre Leitfähigkeit}

1999 wurden von Mai bis September bei jeder untersuchten Pflanzenart fünf Messungen der Transpiration und stomatären Leitfähigkeit auf den FN-Flächen und zwei im August und September/Oktober auf den unbewässerten DN-Flächen durchgeführt. Im Jahr 2000 wurde im gleichen Zeitraum an je vier Tagen parallel auf den FN- und FU-Flächen jeder Art gemessen, um den Effekt der experimentellen Nutzung zu untersuchen. Bei $A$. sparsifolia wurde nur auf der AFN-Fläche gemessen, da bei dieser Art keine Unterschiede im Wasserhaushalt durch die experimentelle Nutzung erwartet wurden.

\section{Alhagi sparsifolia}

Bei Alhagi sparsifolia wurde die erste Messung 1999 mit der „broad leaf“ Kammer des Porometers an einem ausreichend großen Blatt in mittlerer Höhe des Busches durchgeführt. Die Messungen an den anderen Terminen erfolgten in der oberen Krone an Triebspitzen mit kleinen Blättern und Dornen. Die erste Messung ist daher aufgrund der unterschiedlichen Probenbeschaffenheit mit den anderen nur bedingt vergleichbar.

Bei der ersten Messung (15.05.1999) wurden sowohl auf die Blattfläche (nicht dargestellt) als auch auf das Trockengewicht bezogen sehr hohe Transpirations-

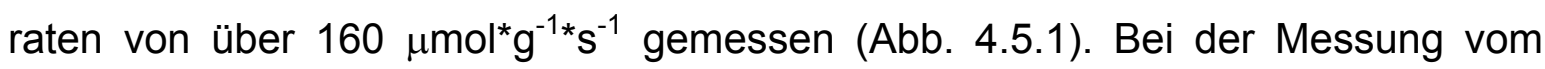
01.06.1999 fällt auf, dass vormittags um den Faktor 10 höhere Transpirationsraten vorlagen als am Nachmittag. Die Transpirationsrate fiel plötzlich ab und erreichte am Nachmittag sehr niedrige Werte. Am 16.06.1999 wurde die geringste Transpiration im Jahresverlauf gemessen. An den meisten Tagen, mit Ausnahme des 01.06., stieg die Transpiration morgens an, erreichte nachmittags ihren Höhepunkt und sank zum Abend wieder ab (Abb. 4.5.1). Bei A. sparsifolia trat bei den Messungen auf der AFN-Fläche eine hochsignifikante Korrelation zwischen Transpirationsmenge (Tagessumme) und dem Mittelwert des VPD $\left(r^{2}=0,968 ; P<0,0001\right)$ sowie dem maximalen VPD $\left(r^{2}=0,805 ; P=0,0025\right)$ auf.

Auf der ADN-Fläche (Abb. 4.5.1) konnte man im August und September 1999 signifikant niedrigere Transpirationswerte als auf der AFN-Fläche feststellen, es kam auf der trockenen Fläche auch zu einer leichten Einschränkung der 
Transpiration am Nachmittag. Da die Messungen an unterschiedlichen Tagen stattfanden, wurden die Strahlungssummen und das VPD der Messtermine verglichen. Obwohl VPD und Strahlung im August bei der Messung auf der ADNFläche höher waren, im September dagegen auf der AFN-Fläche, war doch die Transpiration in beiden Monaten auf der bewässerten Fläche höher.

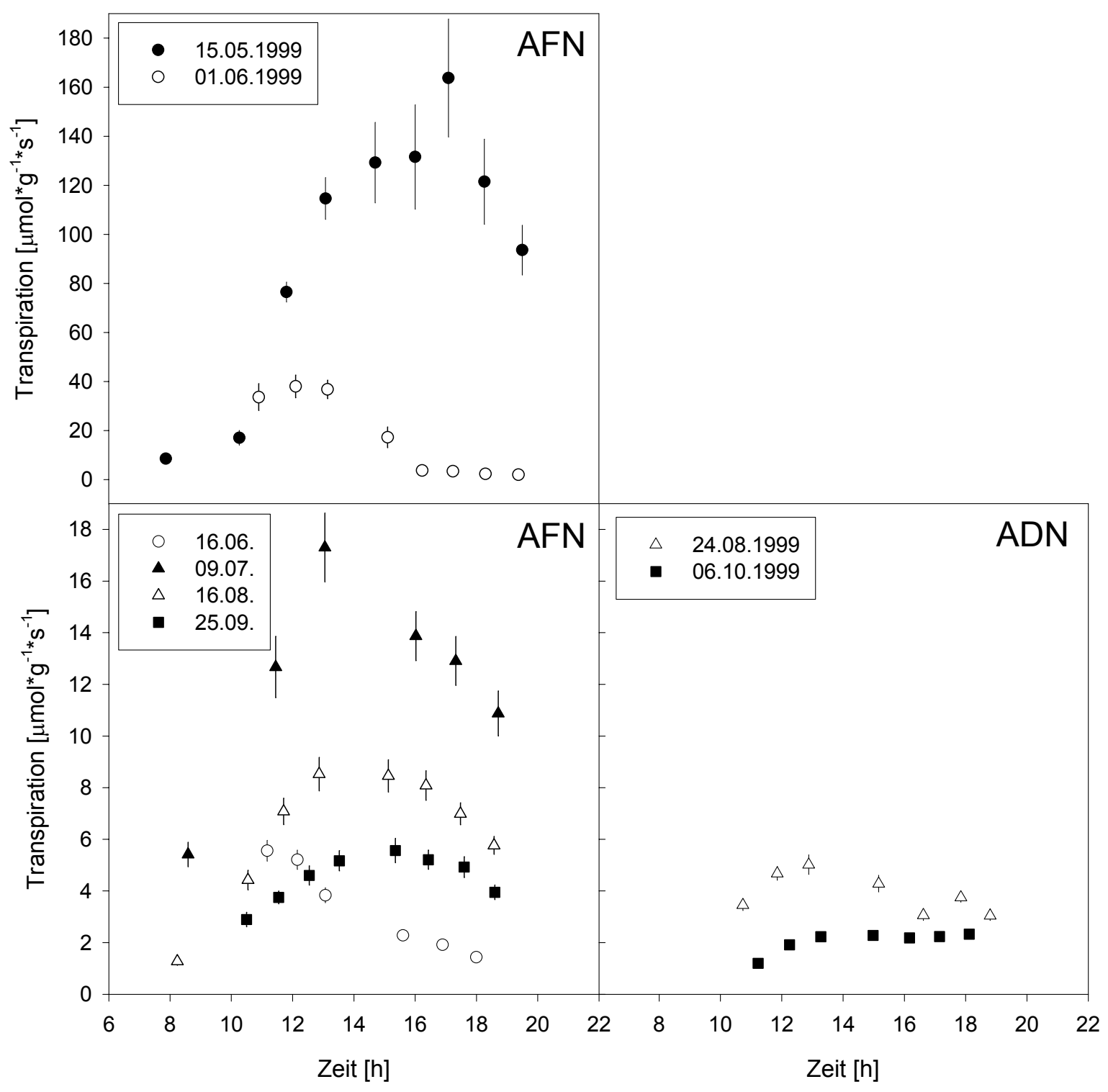

Abb. 4.5.1: Transpiration von Alhagi sparsifolia 1999.

Die Tagesgänge wurden auf der bewässerten ungenutzten Fläche (AFN) und der unbewässerten ungenutzten Fläche (ADN) gemessen. Die Werte sind bezogen auf das Blatttrockengewicht. Das obere Teilbild (15.05. und 01.06.1999) ist in einem anderen Maßstab skaliert. 
1999 wurden im Mai an großen Blättern mit der „broad leaf"-Kammer eine stomatäre Leitfähigkeit von bis zu $3,06 \mathrm{mmol}^{*} \mathrm{~g}^{-1 *} \mathrm{~s}^{-1}$ gemessen (Abb. 4.5.2). Ein sprunghafter Anstieg am Vormittag auf mehr als doppelt so hohe Werte fällt auf.

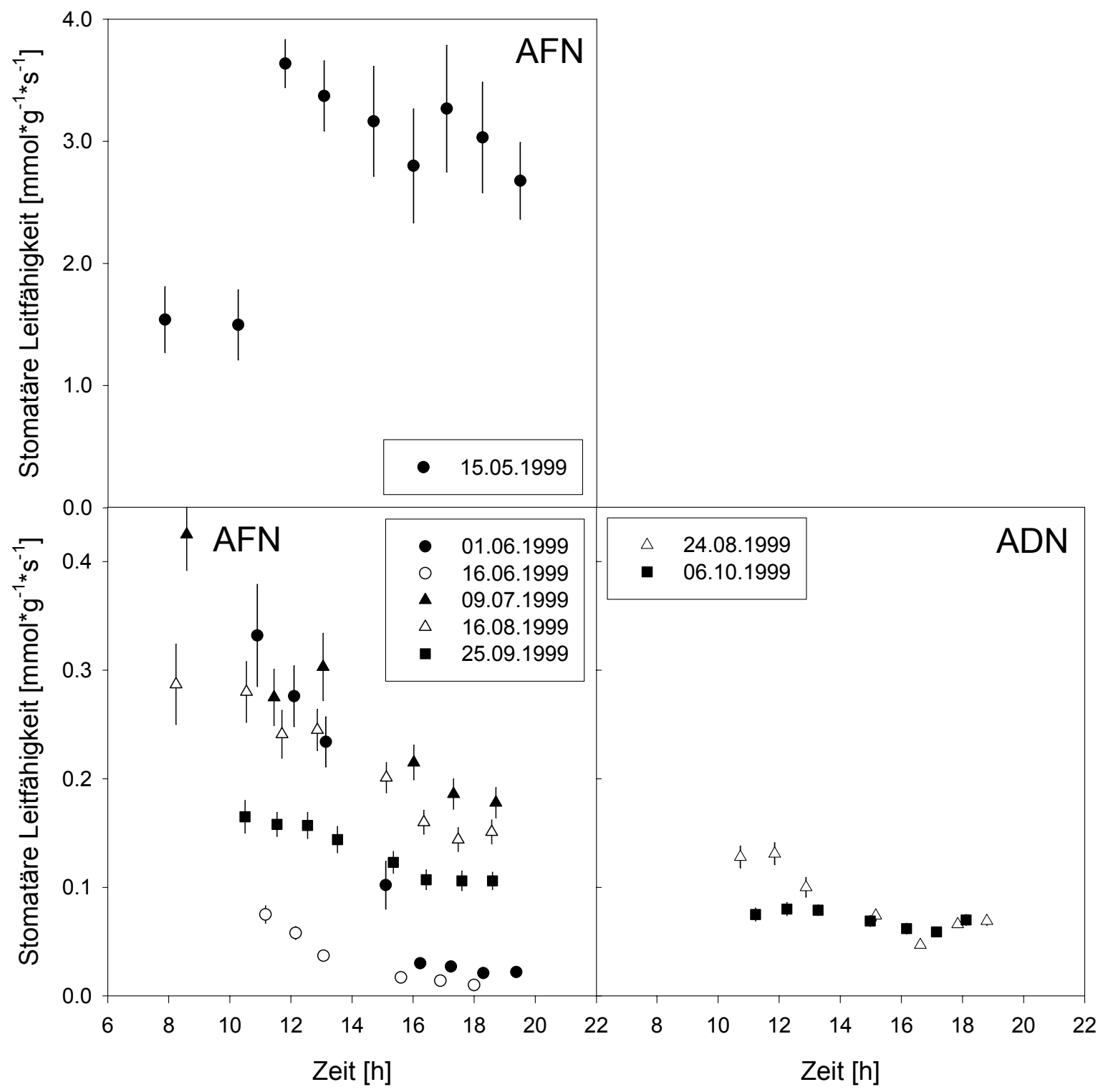

Abb. 4.5.2: Stomatäre Leitfähigkeit von Alhagi sparsifolia 1999.

Die Tagesgänge wurden auf der bewässerten ungenutzten Fläche (AFN) und der unbewässerten ungenutzten Fläche (ADN) gemessen. Die Werte sind bezogen auf das Blatttrockengewicht. Das obere Teilbild (15.05.1999) ist in einem anderen Maßstab skaliert.

Anfang Juni zeigte die Leitfähigkeit einen ungewöhnlichen Verlauf, da sie von einem recht hohen Wert $\left(0,33 \mathrm{mmol}^{*} \mathrm{~g}^{-1 *} \mathrm{~s}^{-1}\right)$ morgens rasch auf sehr niedrige Werte am Nachmittag absank. Auch an den anderen Messterminen war von einer 
maximalen Leitfähigkeit ( $g_{s} \max$ ) am Morgen ein mehr oder weniger schnelles Absinken zu beobachten. Die niedrigste $\mathrm{g}_{\mathrm{s}} \max \mathrm{im}$ Verlauf der Vegetationsperiode wurde mit 0,06 $\mathrm{mmol}^{*} \mathrm{~g}^{-1 *} \mathrm{~s}^{-1}$ Mitte Juni gemessen. Am Messtag im Juli herrschte ein sehr hohes VPD (Abb. 4.4.1), die stomatäre Leitfähigkeit sank sehr rasch ab. Die stomatäre Leitfähigkeit zeigte im Sommer einen klaren Zusammenhang mit dem Wassersättigungsdefizit (VPD) der Luft $\left(r^{2}=0.369\right.$, P = 0.0016). Im September wurden wieder deutlich niedrigere Werte ermittelt und der Tagesgang verlief gleichmäßiger als im Juli und August, was auf beginnende Seneszenz zurückzuführen sein kann (Abb. 4.5.2).

Im Jahr 2000 wurde bei Alhagi sparsifolia nur auf der AFN-Fläche gemessen. Bei der Nutzungsernte hatte keine Auflichtung des Bestandes stattgefunden, da auf der genutzten Teilfläche alle Pflanzen geerntet worden waren. Deswegen wurde auf eine parallele Messung der FN- und FU-Flächen verzichtet.

Im Mai 2000 wurden mit maximal 19,64 $\mu \mathrm{mol}^{*} \mathrm{~g}^{-1 *} \mathrm{~s}^{-1}$ wieder die höchsten Transpirationswerte im Jahresverlauf gemessen (Abb. 4.5.3). Im Juni waren die Messwerte wie schon 1999 am niedrigsten. Im August stiegen die Werte wieder bis auf $11,42 \mu \mathrm{mol}^{*} \mathrm{~g}^{-1 *} \mathrm{~s}^{-1}$ an und sanken im September fast auf das Niveau von Juni ab (Abb. 4.5.3).

Die stomatäre Leitfähigkeit sank 2000 an allen Terminen zum Nachmittag hin ab. Dies geschah gegen Ende der Vegetationsperiode schon gegen $13 \mathrm{Uhr}$, während im Mai nach einem Anstieg am Vormittag erst ab 15 Uhr niedrigere Werte zu beobachten waren (Abb. 4.5.4). Die höchsten Werte der Vegetationsperiode wurden im Mai mit $0,35 \mathrm{mmol}^{*} \mathrm{~g}^{-1 *} \mathrm{~s}^{-1}$ erreicht. Im Juni und August traten im Tagesverlauf nur geringe Änderungen auf, die Werte bewegten sich um 0,2 $\mathrm{mmol}^{*} \mathrm{~g}^{-1 *} \mathrm{~s}^{-1}$. Im September wurden die niedrigsten Nachmittagswerte gemessen. 


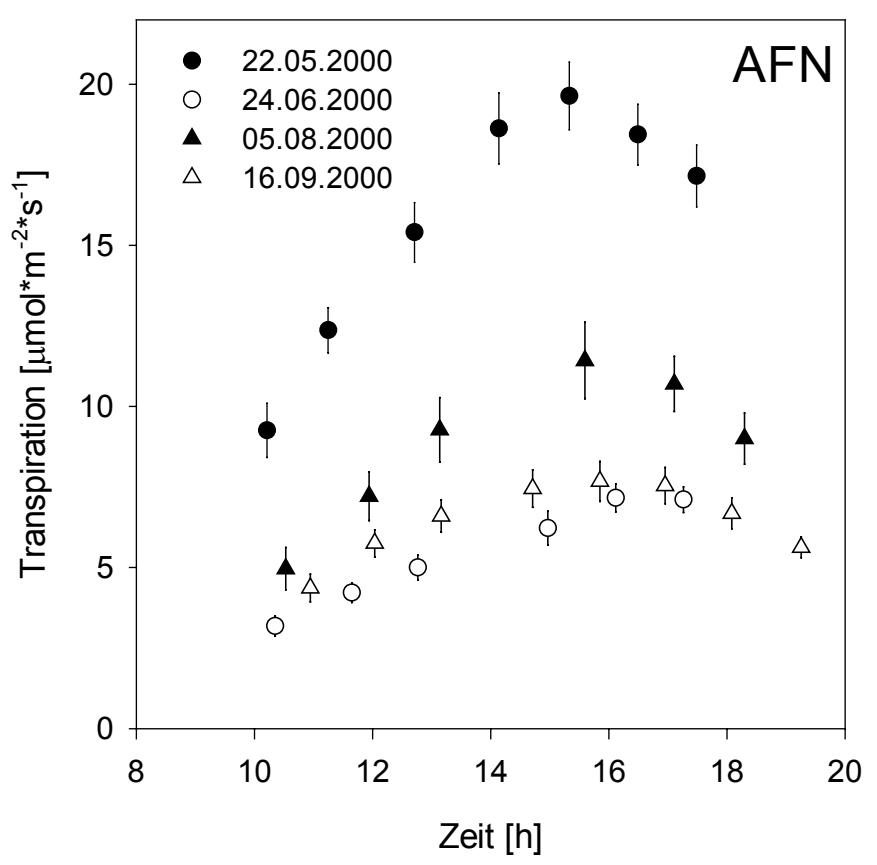

Abb. 4.5.3: Transpiration von Alhagi sparsifolia 2000.

Die Tagesgänge wurden auf der im Vorjahr bewässerten ungenutzten Fläche (AFN) gemessen. Die Werte sind bezogen auf das Blatttrockengewicht.

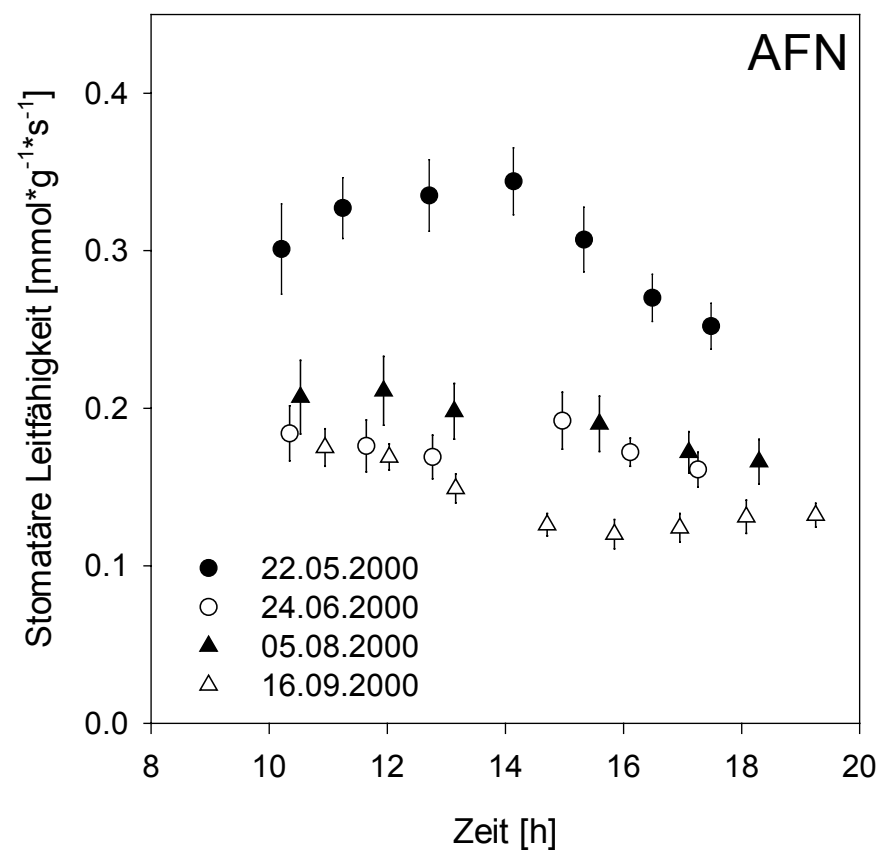

Abb. 4.5.4: Stomatäre Leitfähigkeit von Alhagi sparsifolia 2000.

Die Tagesgänge wurden auf der im Vorjahr bewässerten ungenutzten Fläche (AFN) gemessen. Die Werte sind bezogen auf das Blatttrockengewicht. 


\section{Calligonum caput-medusae}

Bei C. caput-medusae liegt für Mai 1999 keine Messung vor. Bei den Messungen vom 07.06., 12.08. und 23.09. wurde die maximale Transpirationsrate am Mittag oder frühen Nachmittag gemessen, nur bei der Messung am 18.07.1999 trat offenbar eine Mittagsdepression auf (Abb. 4.5.5). Die höchsten flächenbezogenen Transpirationsraten traten auf der CFN-Fläche mit etwa $1 \mathrm{mmol}^{*} \mathrm{~m}^{-2 *} \mathrm{~s}^{-1}$ im September auf. An den anderen Terminen wurden höchstens $0,65 \mathrm{mmol}^{*} \mathrm{~m}^{-2 *} \mathrm{~s}^{-1}$ erreicht (nicht dargestellt). Auf das Trockengewicht bezogen lagen die Transpirationswerte im Juni mit bis zu $6,53 \mu \mathrm{mol}^{*} \mathrm{~g}^{-1 *} \mathrm{~s}^{-1}$ höher als im Juli und August. Die niedrigsten Werte wurden Ende September mit nur $3,72 \mu \mathrm{mol}^{*} \mathrm{~g}^{-1 *} \mathrm{~s}^{-1}$ erreicht (Abb. 4.5.5).

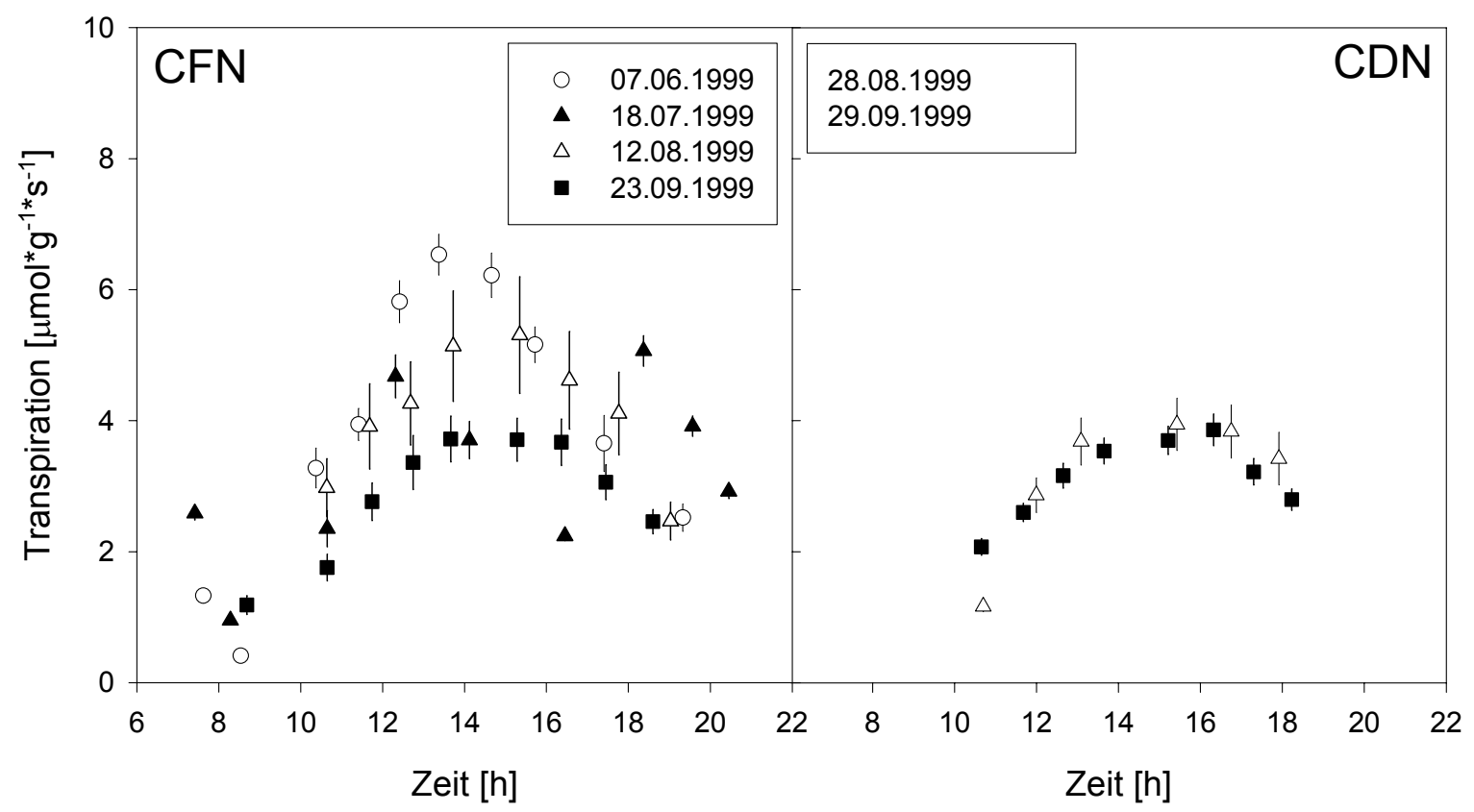

Abb. 4.5.5: Transpiration von Calligonum caput-medusae 1999.

Dargestellt sind die trockengewichtsbezogenen Werte auf der experimentell bewässerten Fläche (CFN) und der trockenen Vergleichsfläche (CDN).

Die Transpirationsraten auf der CDN-Fläche blieben an beiden Terminen Ende August und Ende September 1999 unter 0,5 $\mathrm{mmol}^{*} \mathrm{~m}^{-2 *} \mathrm{~s}^{-1}$ bzW. $4 \mu \mathrm{mol}^{*} \mathrm{~g}^{-1 *} \mathrm{~s}^{-1}$, im August waren die Werte signifikant niedriger als die auf der CFN-Fläche (Abb. 4.5.5). Es war keine Mittagsdepression zu beobachten. Sowohl im August als auch im September waren Strahlungssumme und VPD an den Tagen, an denen auf der 
CFN-Fläche gemessen wurde, kaum höher als bei den Messungen auf der CDNFläche.

Die stomatäre Leitfähigkeit nahm im Tagesverlauf ab (Abb. 4.5.6). Im Juni 1999 wurden mit einer maximalen Leitfähigkeit von $0,035 \mathrm{mmol}^{*} \mathrm{~g}^{-1 *} \mathrm{~s}^{-1}$ extrem niedrige Werte gemessen. Im Juli und September 1999 zeigten die Werte am Morgen mit 0,294 bzw. 0,246 $\mathrm{mmol}^{*} \mathrm{~g}^{-1 *} \mathrm{~s}^{-1}$ ihr Maximum und fielen schnell auf ungefähr ein Drittel des Wertes ab. Im Juli war ein deutlicher Stomataschluss am Nachmittag zu erkennen, der vermutlich auf das sehr hohe VPD $\max$ von 6,35 kPa (mittleres VPD: 3,15 kPa) und auf Messfehler durch Überhitzung der Küvette zurückzuführen ist. Von diesem Tag liegen keine Wasserpotentialmessungen vor, die die Ergebnisse unterstützen können. Der Saftfluss zeigte an diesem Nachmittag keinerlei Einschränkung. Im August stieg die Leitfähigkeit bis mittags auf $0,135 \mathrm{mmol}^{*} \mathrm{~g}^{-1 *} \mathrm{~s}^{-1}$ an und sank erst am Nachmittag ab. Die Tagesgänge auf der CDN-Fläche zeigten einen ähnlichen Verlauf mit teilweisem Schließen der Stomata nachmittags (Abb. 4.5.6). Während im August vergleichbare Werte wie auf der CFN-Fläche gemessen wurden, lag die stomatäre Leitfähigkeit im September auf der CDN-Fläche signifikant höher.

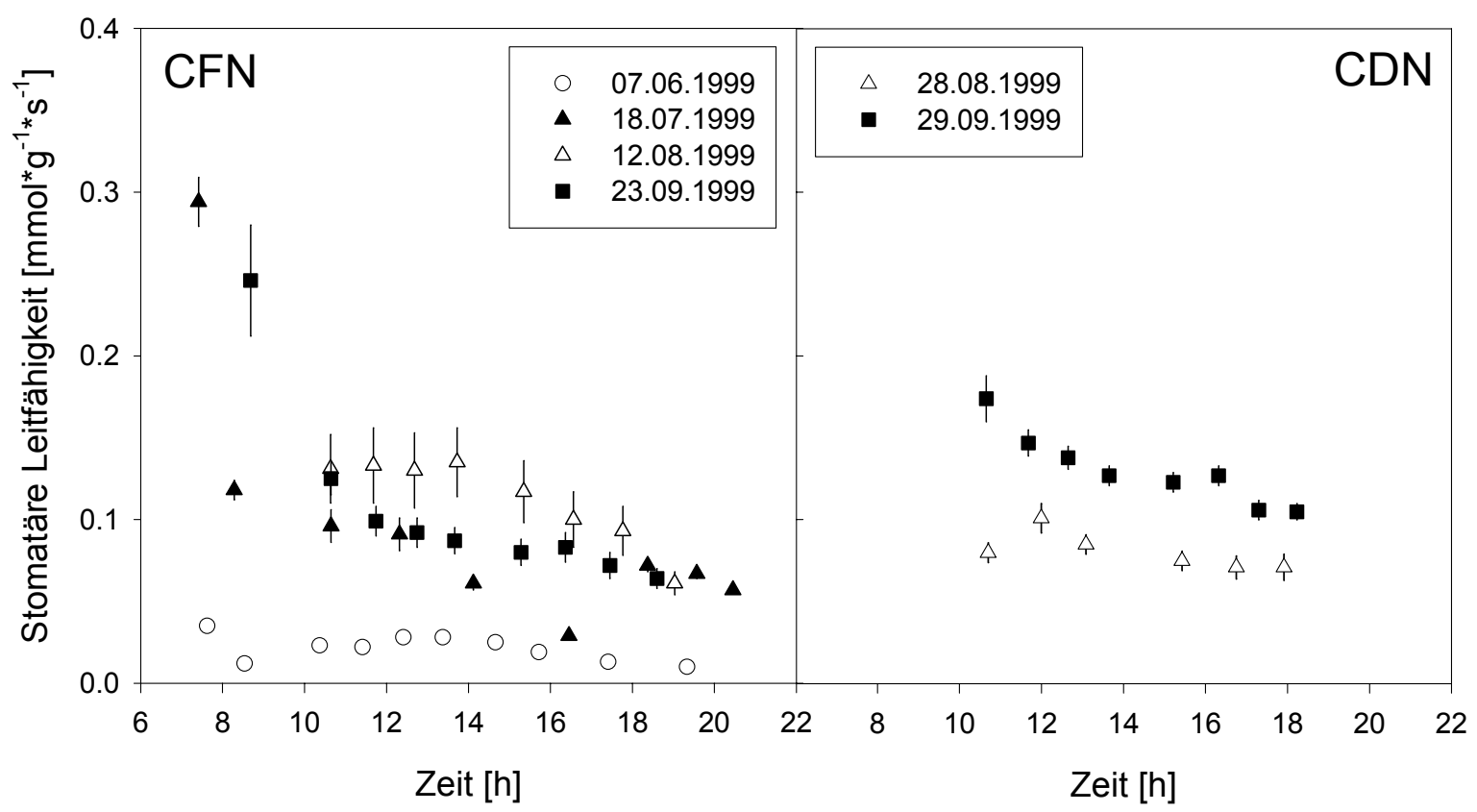

Abb. 4.5.6: Stomatäre Leitfähigkeit von Calligonum caput-medusae 1999.

Dargestellt sind die trockengewichtsbezogenen Werte auf der experimentell bewässerten (CFN) und der trockenen Vergleichsfläche (CDN). 
Auf der CFN-Fläche wurde 2000 an den gleichen Büschen gemessen wie 1999. Parallel erfolgte eine Messung auf der experimentell genutzten CFU-Fläche. Auf beiden Teilflächen wurden im Mai mit etwa $1 \mathrm{mmol}^{*} \mathrm{~m}^{-2 *} \mathrm{~s}^{-1}$ die höchsten Transpirationswerte erreicht. Mit unter $0,5 \mathrm{mmol}^{*} \mathrm{~m}^{-2 *} \mathrm{~s}^{-1}$ waren im Juni die im Jahresverlauf geringsten Werte zu beobachten (nicht dargestellt), bei der CFU-Fläche trat eine Mittagsdepression auf. Auf das Trockengewicht bezogen lag die Messung im Mai mit $12,74 \mu \mathrm{mol}^{*} \mathrm{~g}^{-1 *} \mathrm{~s}^{-1}$ auf der ungenutzten und $17,84 \mu \mathrm{mol}^{*} \mathrm{~g}^{-1 *} \mathrm{~s}^{-1}$ auf der genutzten Fläche deutlich höher als die anderen Messungen, die nur Transpirationsraten von $4,91-5,89 \mu \mathrm{mol}^{*} \mathrm{~g}^{-1 *} \mathrm{~s}^{-1}$ (CFN und CFU) erreichten. Bei C. caputmedusae waren im Mai VPD und Wasserverbrauch auf der genutzten Fläche höher, im weiteren Jahresverlauf waren die Flächen gleich oder die ungenutzte wies höhere Werte auf. An keinem der Termine unterschieden sich die transpirierten Wassermengen auf den beiden Teilflächen jedoch signifikant. C. caput-medusae zeigte keine Abhängigkeit vom Wassersättigungsdefizit und nur eine schwache, aber nicht signifikante, Abhängigkeit von der Strahlungssumme.

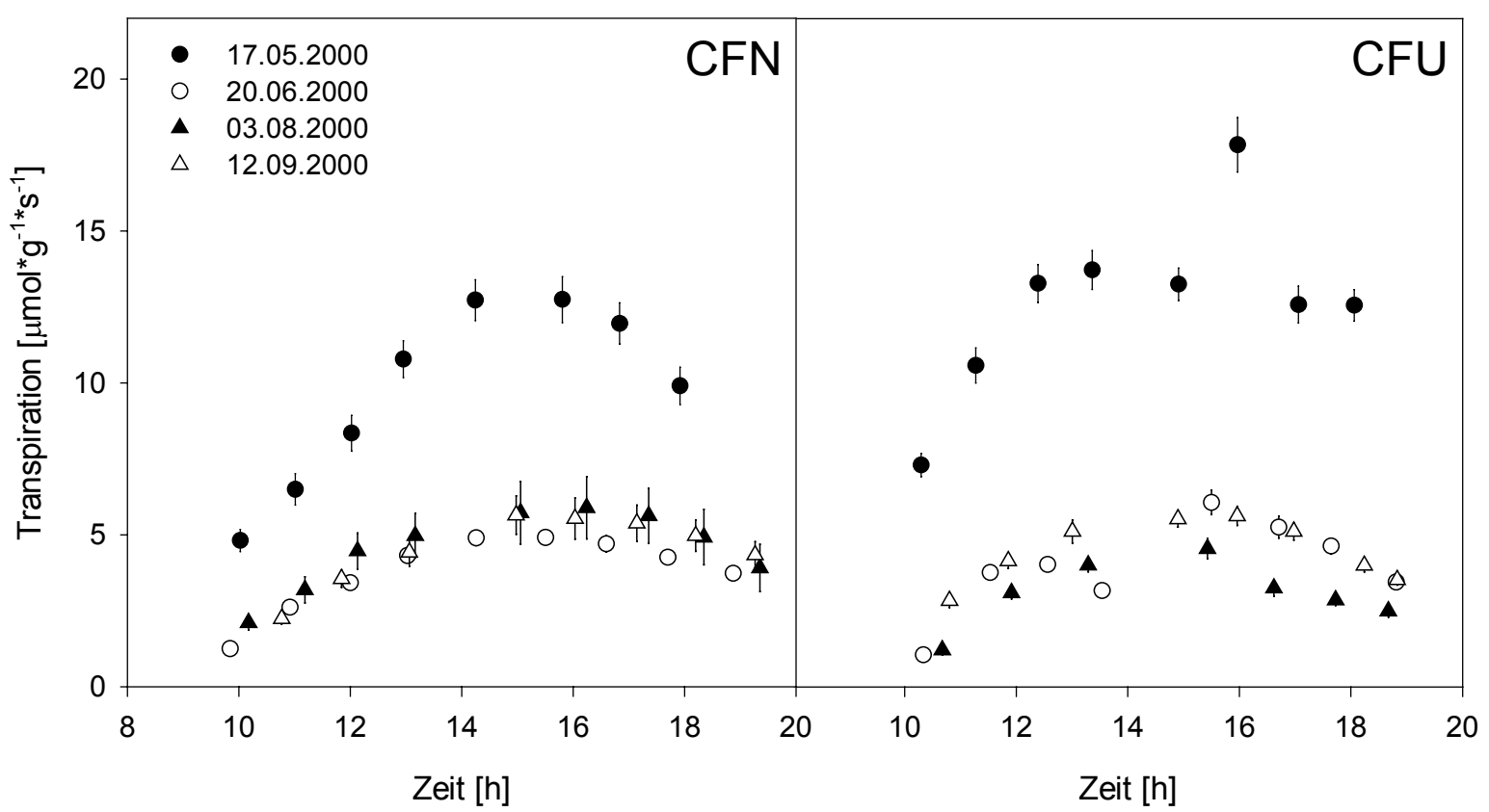

Abb. 4.5.7: Transpiration von Calligonum caput-medusae 2000

Die Tagesgänge wurden parallel auf der im Vorjahr bewässerten ungenutzten (CFN) und genutzten (CFU) Teilfläche gemessen. Die Werte sind bezogen auf das Blatttrockengewicht. 
Die stomatäre Leitfähigkeit lag im Mai deutlich über denen der anderen Monate. Wie schon 1999 wurden die geringsten Leitfähigkeiten der Vegetationsperiode im Juni gemessen. Die Augustmessung wies eine vergleichbare Größenordnung mit August 1999 auf, während die Septemberwerte 2000 etwas höher lagen als im Vormonat und auch höher als im September des Vorjahres. Während die Tagesgänge im Mai und August 2000 wie schon 1999 zum Nachmittag hin absanken, zeigte sich im Juni ein sehr flacher Verlauf. Auch im September wurde ein im Tagesverlauf gleichbleibender Wert gemessen.

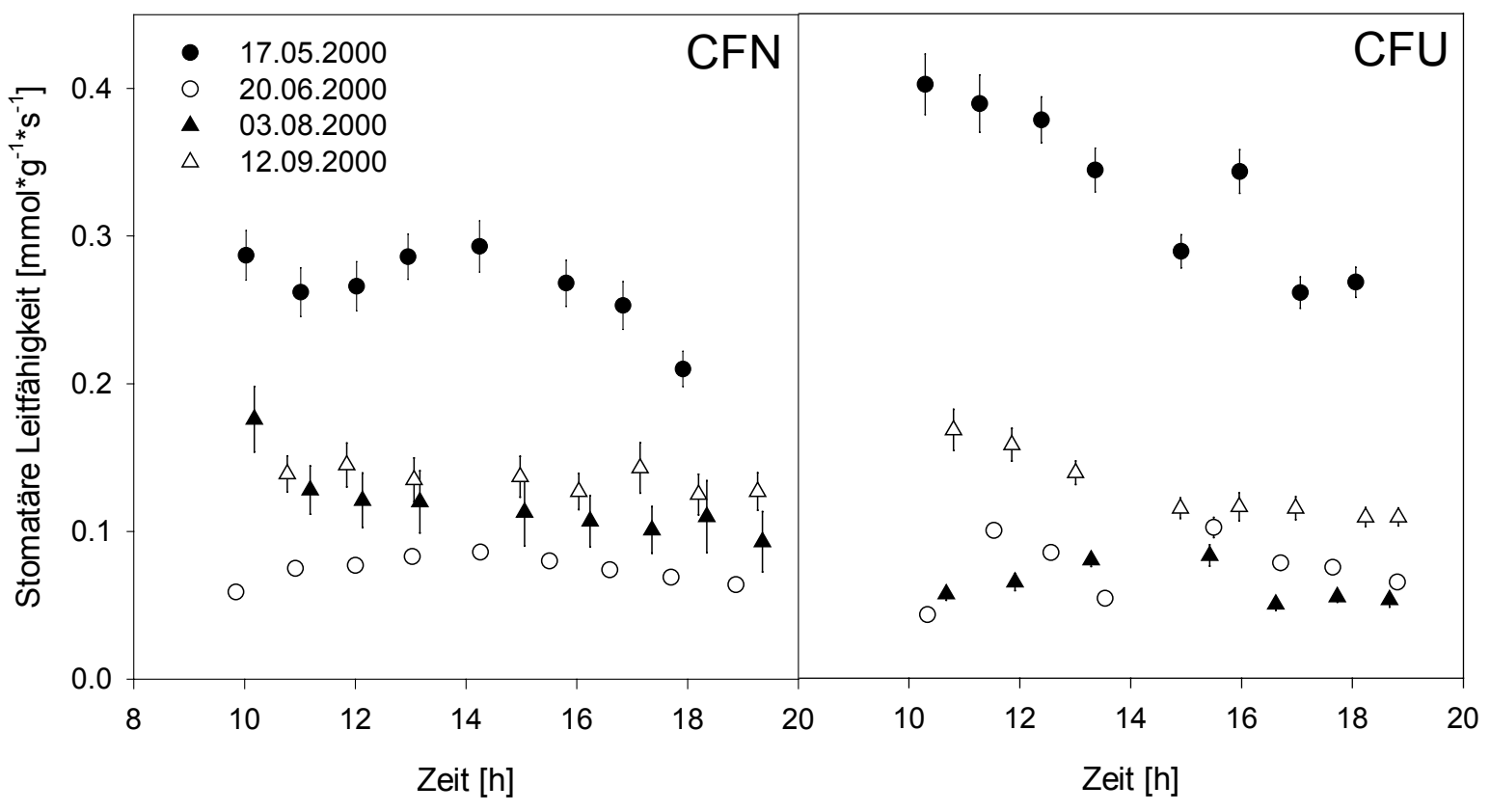

Abb. 4.5.8: Stomatäre Leitfähigkeit von Calligonum caput-medusae 2000.

Dargestellt sind die trockengewichtsbezogenen Werte auf der experimentell bewässerten ungenutzten (CFN) und der genutzten Vergleichsfläche (CFU).

\section{Tamarix ramosissima}

Die erste Messung im Mai $1999\left(E_{\max }=34,85 \mu \mathrm{mol}^{*} \mathrm{~g}^{-1 *} \mathrm{~s}^{-1}\right)$ zeigte mit Abstand höhere Werte als die Messungen später im Jahr (Abb. 4.5.9). Die Messungen im Juni und August erreichten mit etwa $6 \mu \mathrm{mol}^{*} \mathrm{~g}^{-1 *} \mathrm{~s}^{-1}$ ähnliche Größenordnungen, ebenso sind die Messungen im Juli und September $\left(2,92 \mu \mathrm{mol}^{*} \mathrm{~g}^{-1 *} \mathrm{~s}^{-1}\right)$ vergleichbar. Die Transpirationsraten auf der unbewässerten Tamarix-Fläche (TD) erreichten dagegen Anfang September nur 2,65 $\mu \mathrm{mol}^{*} \mathrm{~g}^{-1 *} \mathrm{~s}^{-1}$ und im Oktober nur $1,66 \mu \mathrm{mol}^{*} \mathrm{~g}^{-1 *} \mathrm{~s}^{-1}$ (Abb. 4.5.9). Damit unterscheiden sie sich signifikant von den 
Messwerten im August und September auf der TF-Fläche. Vergleicht man die Witterungsbedingungen an den Messtagen, so fällt auf, dass während der Messungen auf der TFN-Fläche in beiden Monaten ein höheres VPD herrschte als auf der TD-Fläche. Für T. ramosissima bestand allerdings, wenn man die Messungen auf der bewässerten Fläche betrachtete, keine signifikante Korrelation zwischen der Transpirationsmenge und dem mittleren VPD.

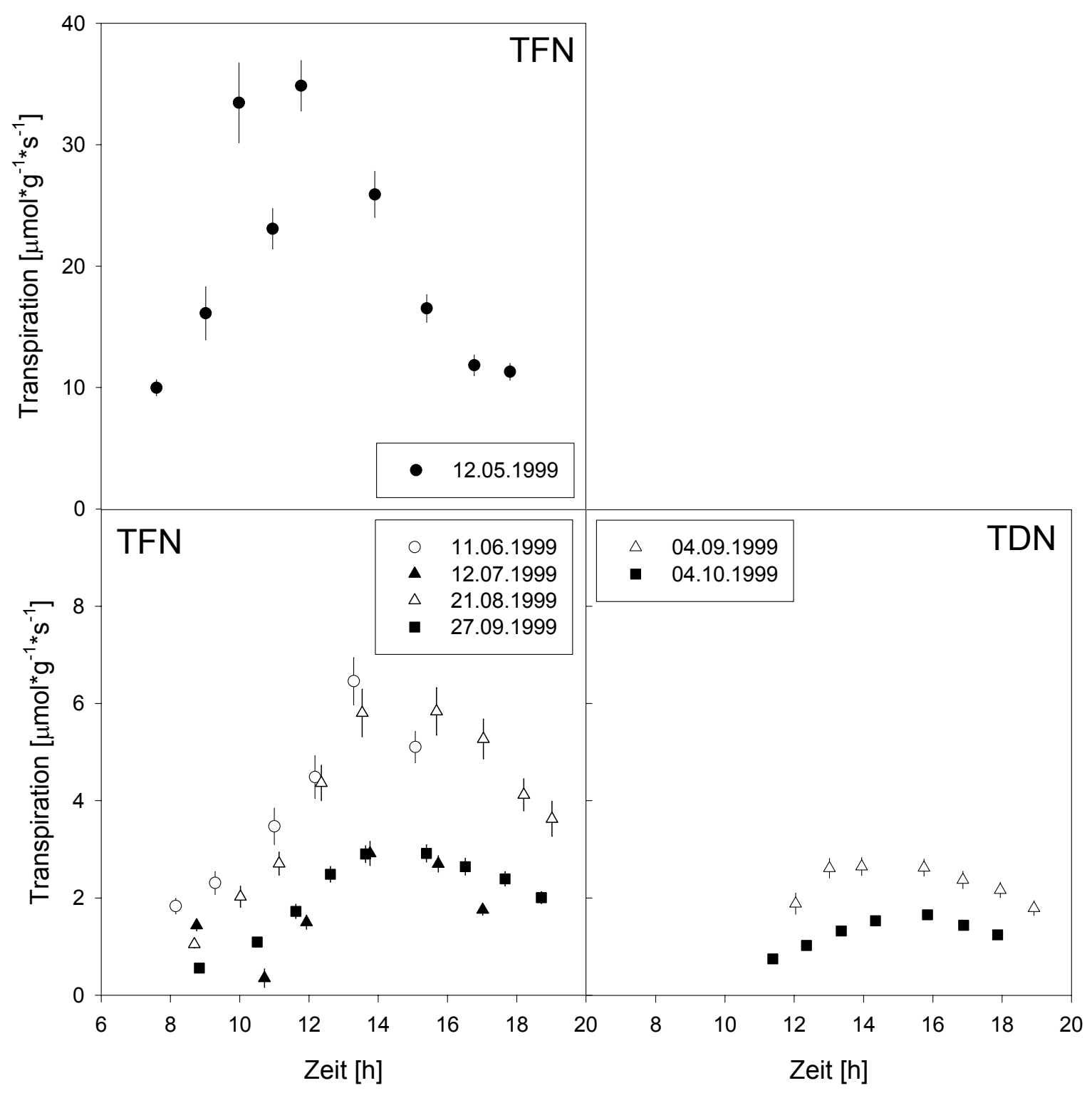

Abb. 4.5.9: Transpiration von Tamarix ramosissima 1999.

Dargestellt sind die trockengewichtsbezogenen Werte auf der experimentell bewässerten ungenutzten (TFN) und der unbewässerten Vergleichsfläche (TDN). 
Bei T. ramosissima war im Mai 1999 mit 0,33 $\mathrm{mmol}^{*} \mathrm{~g}^{-1 *} \mathrm{~s}^{-1}$ eine relativ hohe stomatäre Leitfähigkeit am Morgen zu beobachten (Abb. 4.5.10). Zum Nachmittag hin fiel die Leitfähigkeit ab. Die Messung im Juni zeigte mit nur 0,077 $\mathrm{mmol}^{*} \mathrm{~g}^{-1 *} \mathrm{~s}^{-1}$ die geringste maximale Leitfähigkeit. Da an diesem Tag wegen eines Sturms die Messung abgebrochen werden musste, ist er nur bedingt mit den anderen Tagen vergleichbar. Im Juli und August wurden mit $0,135 \mathrm{mmol}^{*} \mathrm{~g}^{-1 *} \mathrm{~s}^{-1}$ mittlere Werte erreicht, Ende September stieg die Leitfähigkeit nicht über $0,1 \mathrm{mmol}^{*} \mathrm{~g}^{-1 *} \mathrm{~s}^{-1}$.

Auf der unbewässerten TD-Fläche wurden an beiden Messterminen mit maximal $0,067 \mathrm{mmol}^{*} \mathrm{~g}^{-1 *} \mathrm{~s}^{-1}$ eine sehr niedrige Leitfähigkeit festgestellt.

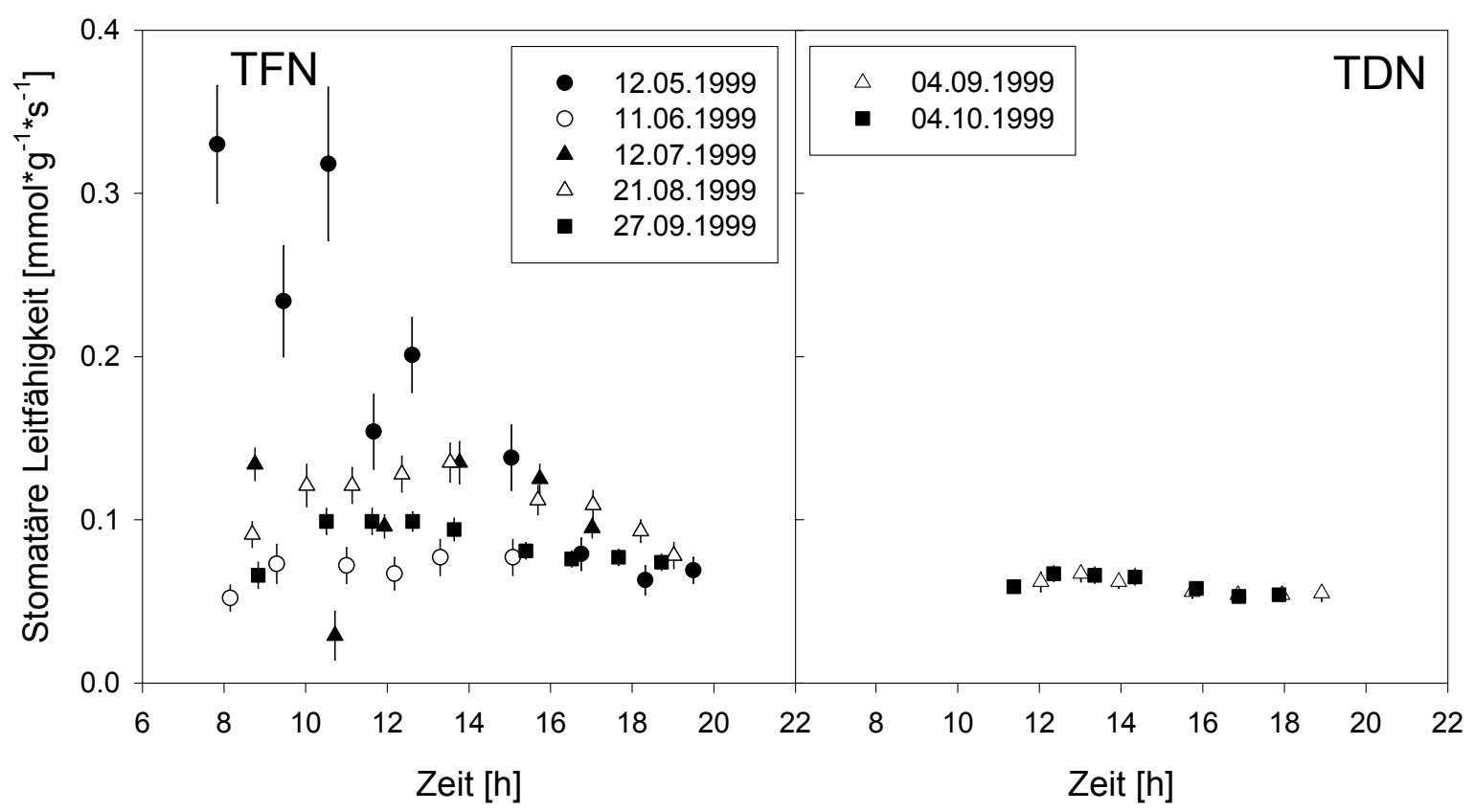

Abb. 4.5.10: Stomatäre Leitfähigkeit von Tamarix ramosissima 1999.

Dargestellt sind die trockengewichtsbezogenen Werte auf der experimentell bewässerten ungenutzten (TFN) und der unbewässerten Vergleichsfläche (TDN).

Im Jahr 2000 konnten auf der TFN-Fläche nur zwei Messungen (Mai und September) durchgeführt werden, da die Tamarisken während der anderen Monate vollständig kahlgefressen waren (siehe Kapitel 4.4). Es fällt auf, dass auf der TFU-Fläche in beiden Monaten ein signifikant höherer Wasserverbrauch durch Transpiration gemessen wurden als auf der TFN-Fläche, im September ist auch das VPD höher, im Mai dagegen niedriger als auf der ungenutzten Fläche. 
Auf der ungenutzten Fläche lag die Transpirationsrate zwar im Mai höher als im September (Abb. 4.5.11), aber die maximale Transpirationsrate im Mai 2000 erreicht weniger als ein Drittel des Wertes von 1999. Auf der genutzten Fläche (TFU) ist dagegen im September ein höherer Wasserverbrauch festzustellen als im Mai. Im Gegensatz zur TFN-Fläche ist im Mai eine deutliche Mittagsdepression zu erkennen.

Im Mai 2000 wurden auf der TFN-Fläche niedrigere stomatäre Leitfähigkeiten gemessen als im Mai 1999. Das Absinken der Leitfähigkeit am Nachmittag war auf der TFN-Fläche eher schwach ausgeprägt, dort blieb die Leitfähigkeit auf einem gleichmäßigen Niveau (Abb. 4.5.12). Im September 2000 lagen die Werte etwas höher als 1999. Da der letzte Messtermin 2000 fast zwei Wochen früher stattfand als im Vorjahr, spielen vermutlich Seneszenzeffekte eine geringere Rolle als 1999. Auf der genutzten TFU-Fläche lag 2000 an beiden Terminen die Leitfähigkeit signifikant höher als auf der TFN-Fläche und zeigte ein deutliches Absinken am Nachmittag.

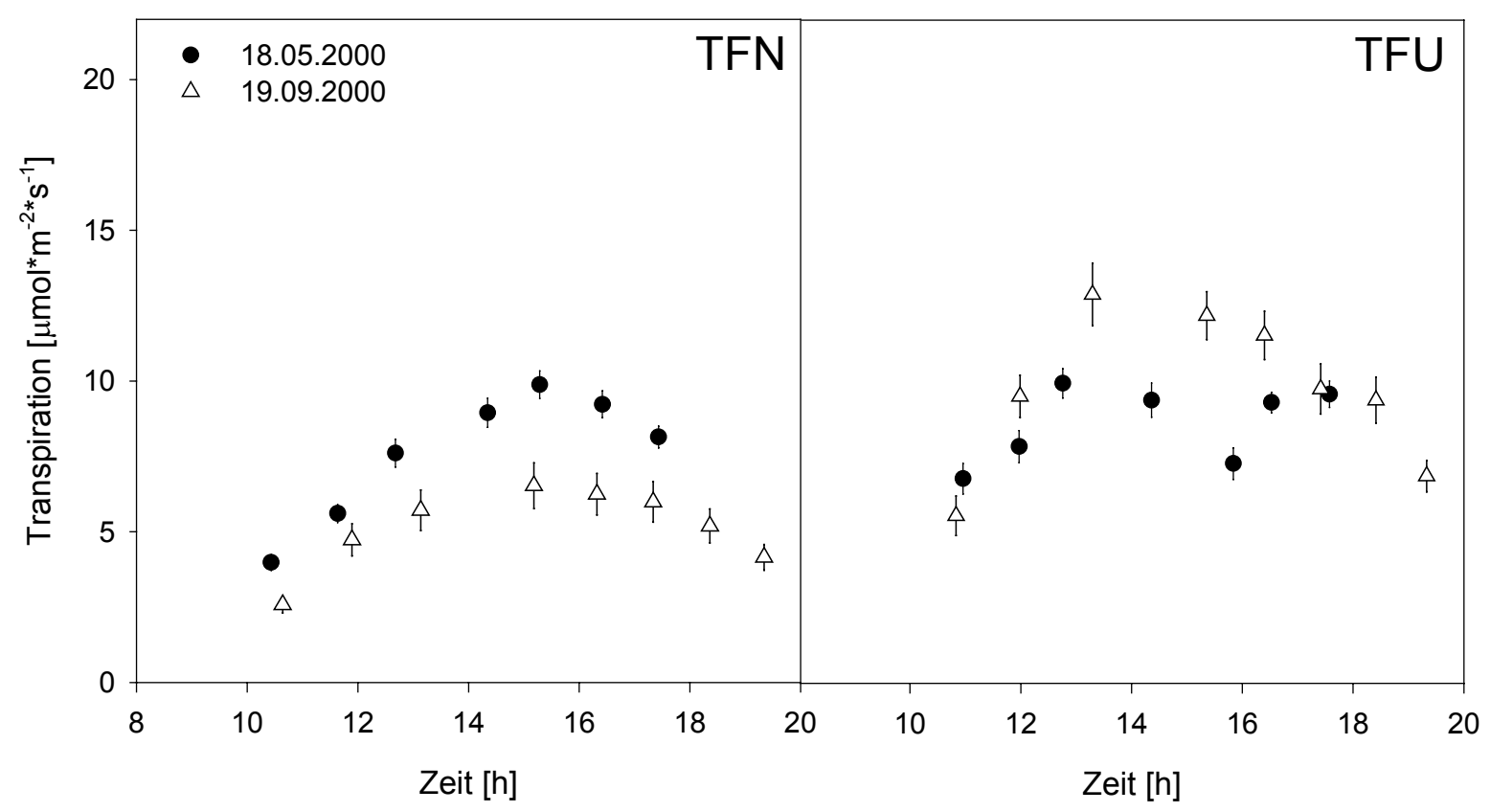

Abb. 4.5.11: Transpiration von Tamarix ramosissima 2000

Die Tagesgänge wurden parallel auf der im Vorjahr bewässerten ungenutzten (TFN) und genutzten (TFU) Teilfläche gemessen. Die Werte sind bezogen auf das Blatttrockengewicht. 


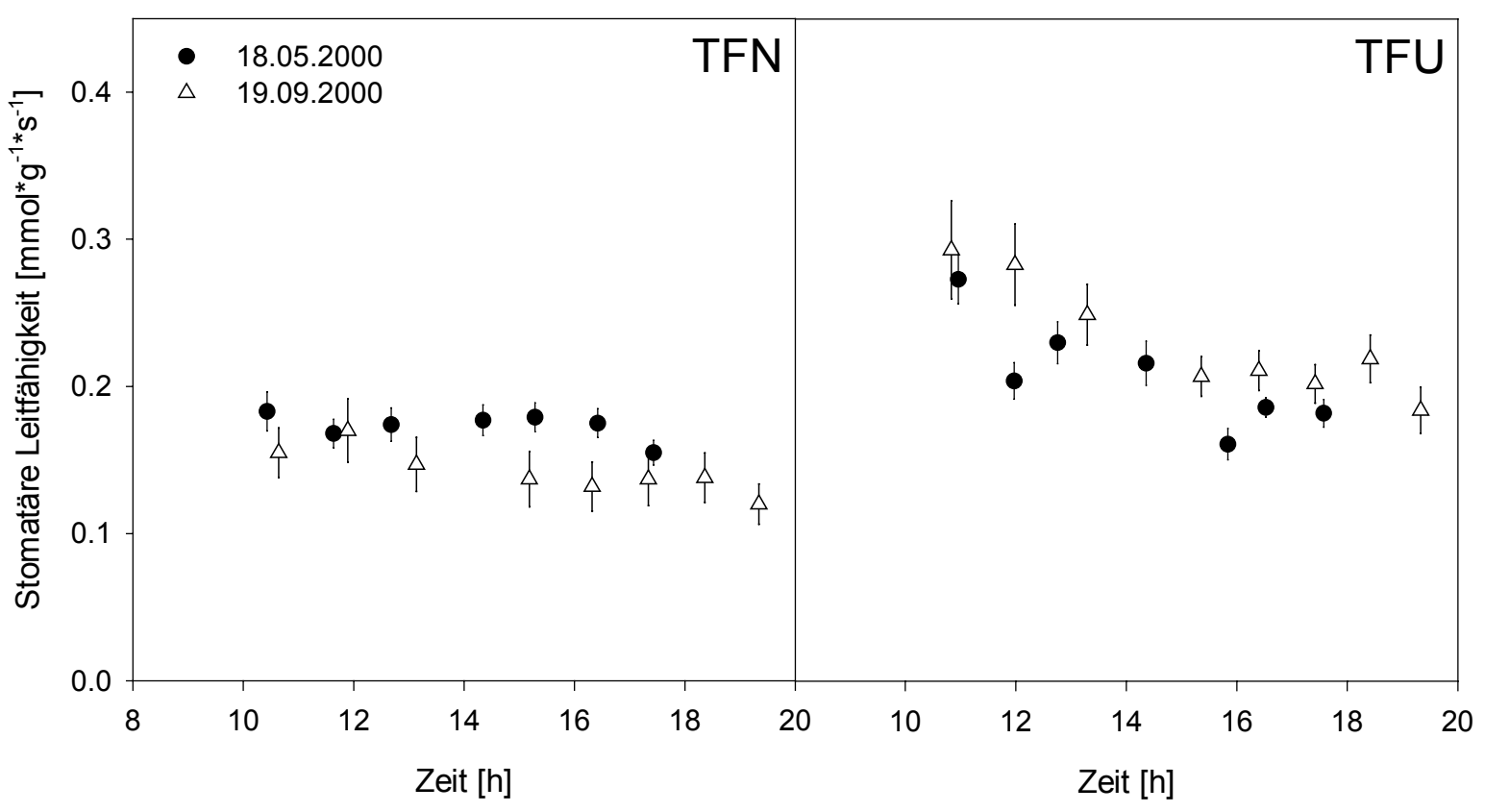

Abb. 4.5.12: Stomatäre Leitfähigkeit von Tamarix ramosissima 2000.

Dargestellt sind die trockengewichtsbezogenen Werte auf der im Vorjahr experimentell bewässerten ungenutzten (TFN) und der genutzten Vergleichsfläche (TFU).

\section{Populus euphratica}

Bei allen Transpirationsmessungen auf der PFN-Fläche 1999 wurden die höchsten Werte mittags erreicht (Abb. 4.5.13). Während die maximalen Werte im Mai nur 2,5 $\mathrm{mmol}^{*} \mathrm{~m}^{-2 *} \mathrm{~s}^{-1}$ betrugen, stiegen sie im Jahresverlauf immer weiter an und erreichten im August bis zu $5 \mathrm{mmol}^{*} \mathrm{~m}^{-2 *} \mathrm{~s}^{-1}$. Im September fiel die Transpirationsrate stark $a b$, was auf beginnende Blattseneszenz schließen lässt. Es wurde kein Zusammenhang zwischen Transpirationsmenge und VPD gefunden, nur eine sehr schwache Abhängigkeit von der Strahlungssumme $\left(r^{2}=0,363 ; P=0,0293\right)$. Auf der PDN-Fläche liegt nur eine Messung Ende August vor. Diese ist signifikant niedriger als die auf der PF-Fläche im gleichen Monat.

Im Untersuchungsjahr 1999 wurden bei $P$. euphratica die höchsten stomatären Leitfähigkeiten der vier untersuchten Arten gemessen. Die Leitfähigkeit stieg morgens an und erreichte am Vormittag den höchsten Wert (Abb. 4.5.14). Danach war ein schnelle Reduktion der Leitfähigkeit zu beobachten. Eine Aus-nahme 
bildete die Messung auf der PFN-Fläche im August 1999: an diesem Tag folgte die stomatäre Leitfähigkeit der Strahlung und zeigte mittags maximale Werte.

Die maximale stomatäre Leitfähigkeit erreichte während der Vegetationsperiode Werte zwischen $1,06 \mathrm{mmol}^{*} \mathrm{~g}^{-1 *} \mathrm{~s}^{-1}$ (Juni) und $1,15 \mathrm{mmol}^{*} \mathrm{~g}^{-1 *} \mathrm{~s}^{-1}$ (Juli). Nur im September lagen die maximalen Werte mit $0,3 \mathrm{mmol}^{*} \mathrm{~g}^{-1 *} \mathrm{~s}^{-1}$ deutlich niedriger, etwa auf dem Niveau der nachmittäglichen Leitfähigkeiten während der Sommermonate (Abb. 4.5.14). Bei $P$. euphratica fällt auf, dass die stomatäre Leitfähigkeit im Laufe der Vegetationsperiode 1999 immer weniger streng reguliert wurde. Während im Mai die Leitfähigkeit schon vormittags steil abfiel, wurde sie im Juli auch mittags auf einem höheren Niveau gehalten und schließlich im August erst am Nachmittag eingeschränkt.

Bei der Messung auf der unbewässerten Fläche Ende August lag die Leitfähigkeit mit maximal $0,55 \mathrm{mmol}^{*} \mathrm{~g}^{-1 *} \mathrm{~s}^{-1}$ recht niedrig.

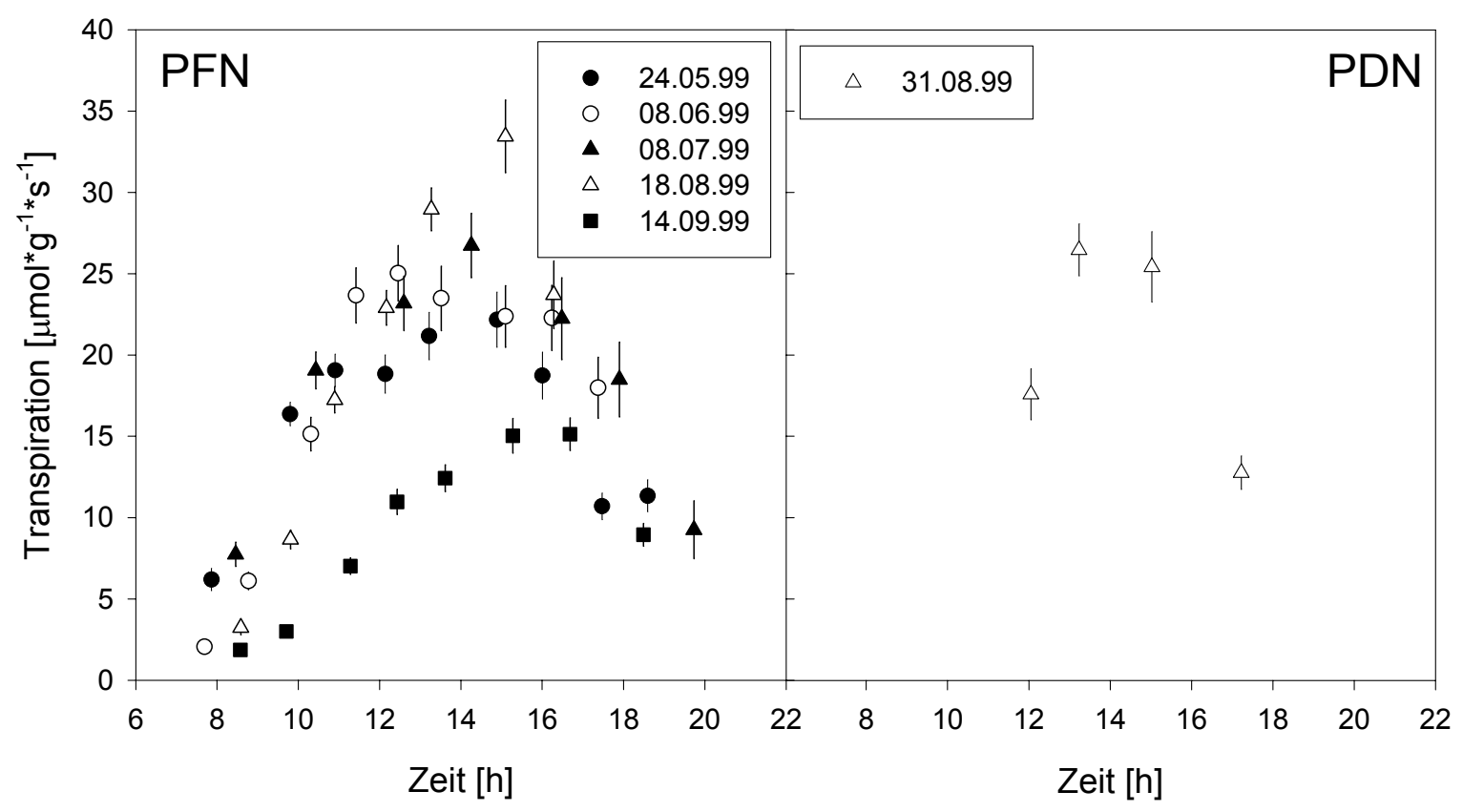

Abb. 4.5.13: Transpiration von Populus euphratica 1999.

Dargestellt sind die trockengewichtsbezogenen Werte auf der experimentell bewässerten ungenutzten (PFN) und der unbewässerten Vergleichsfläche (PDN). 


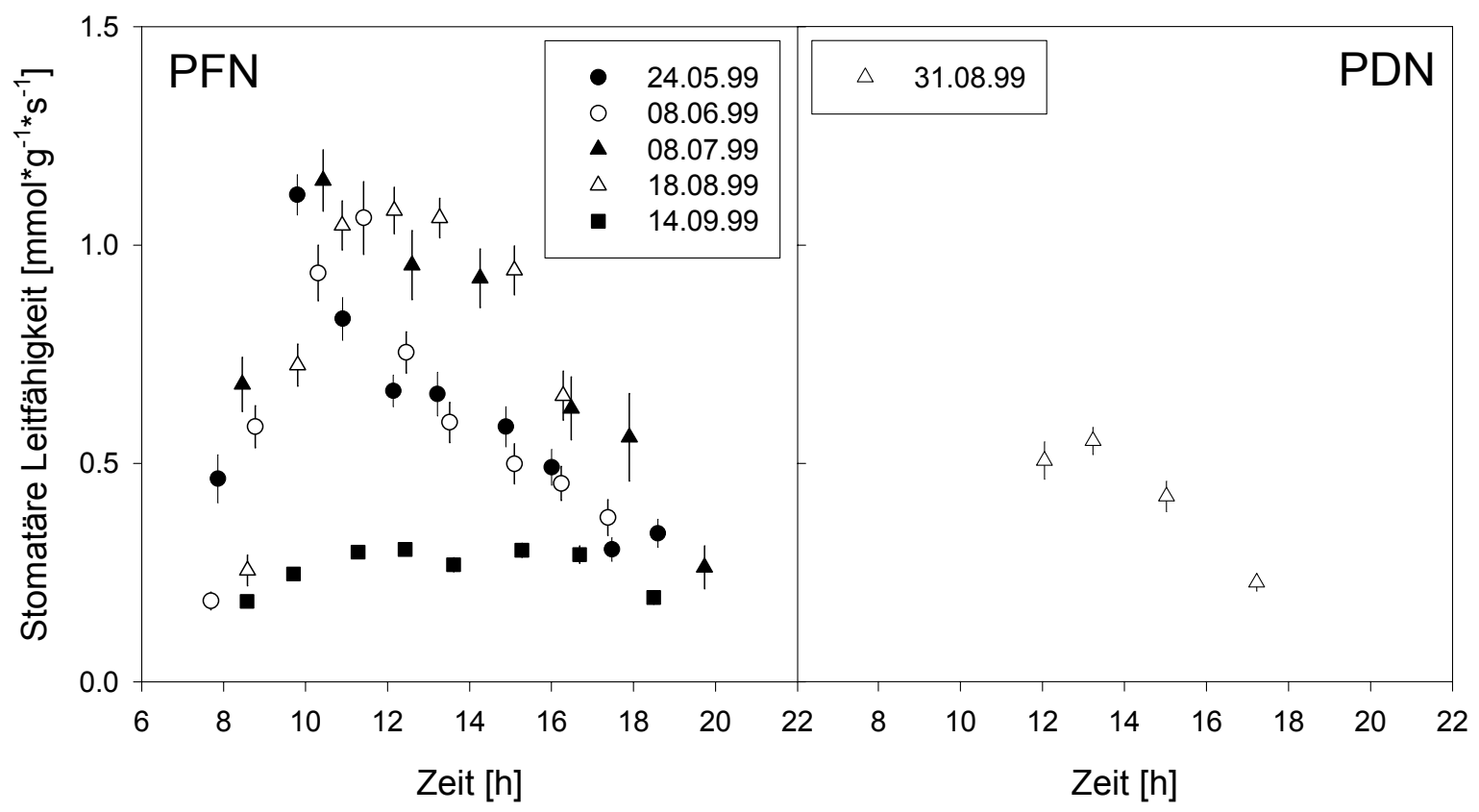

Abb. 4.5.14: Stomatäre Leitfähigkeit von Populus euphratica 1999.

Dargestellt sind die trockengewichtsbezogenen Werte auf der experimentell bewässerten ungenutzten (PFN) Fläche und der unbewässerten Kontrollfläche (PDN).

Die Messungen erfolgten 2000 auf der im Vorjahr bewässerten ungenutzten und genutzten Fläche. Für Mai 2000 liegt keine Messung von der PFU-Fläche vor.

$P$. euphratica zeigte im Mai und Juli 2000 auf der ungenutzten Fläche ein leichtes Absinken der Transpirationsrate auf ein niedrigeres Niveau am Nachmittag, nicht aber später im Jahr (Abb. 4.5.15). Auf der PFN-Fläche wurden wie schon $1999 \mathrm{im}$ Frühjahr mit $25 \mu \mathrm{mol}^{*} \mathrm{~g}^{-1 *} \mathrm{~s}^{-1}$ die höchsten, im Juli 2000 mit maximal $15 \mu \mathrm{mol}^{*} \mathrm{~g}^{-1 *} \mathrm{~s}^{-1}$ die niedrigsten Werte erreicht. Auf der genutzten PFU-Fläche dagegen wurden die mit $27 \mu \mathrm{mol}^{*} \mathrm{~g}^{-1 *} \mathrm{~s}^{-1}$ höchsten Werte im August gemessen, an diesem Tag war die transpirierte Wassermenge signifikant höher als auf der nicht genutzten Fläche. An den anderen Tagen gab es keine signifikanten Unterschiede im Wasserverbrauch. Im Mai 2000 wurden auf der PFN-Fläche deutlich niedrigere Leitfähigkeiten gemessen als im Vorjahr. Eine Vergleichsmessung von der genutzten Fläche liegt nicht vor. Im Juni sank die Leitfähigkeit wie auch 1999 schon am Vormittag ab, während später im Jahr das teilweise Schließen der Stomata erst mittags erfolgt. Die Leitfähigkeit erreichte im Juni auf beiden Teilflächen vergleichbare Werte, im August aber war sie auf der PFU-Fläche und im September auf der PFN-Fläche signifikant höher. Insgesamt wurden in der Vegetationsperiode 2000 niedrigere Leitfähigkeiten gemessen als 1999. 


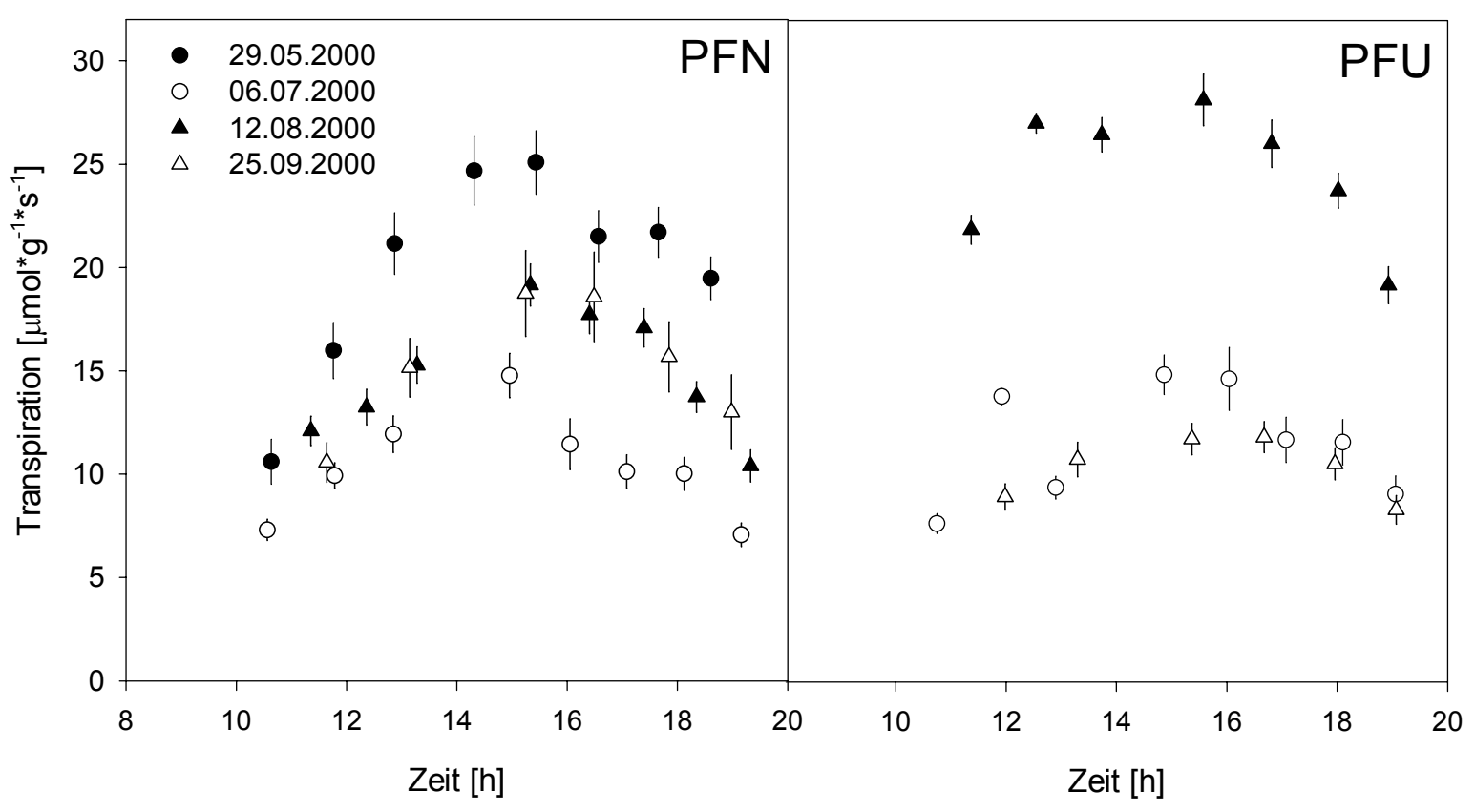

Abb. 4.5.15: Transpiration von Populus euphratica 2000.

Die trockengewichtsbezogenen Werte wurden parallel auf der im Vorjahr bewässerten ungenutzten (PFN) und genutzten (PFU) Fläche gemessen.

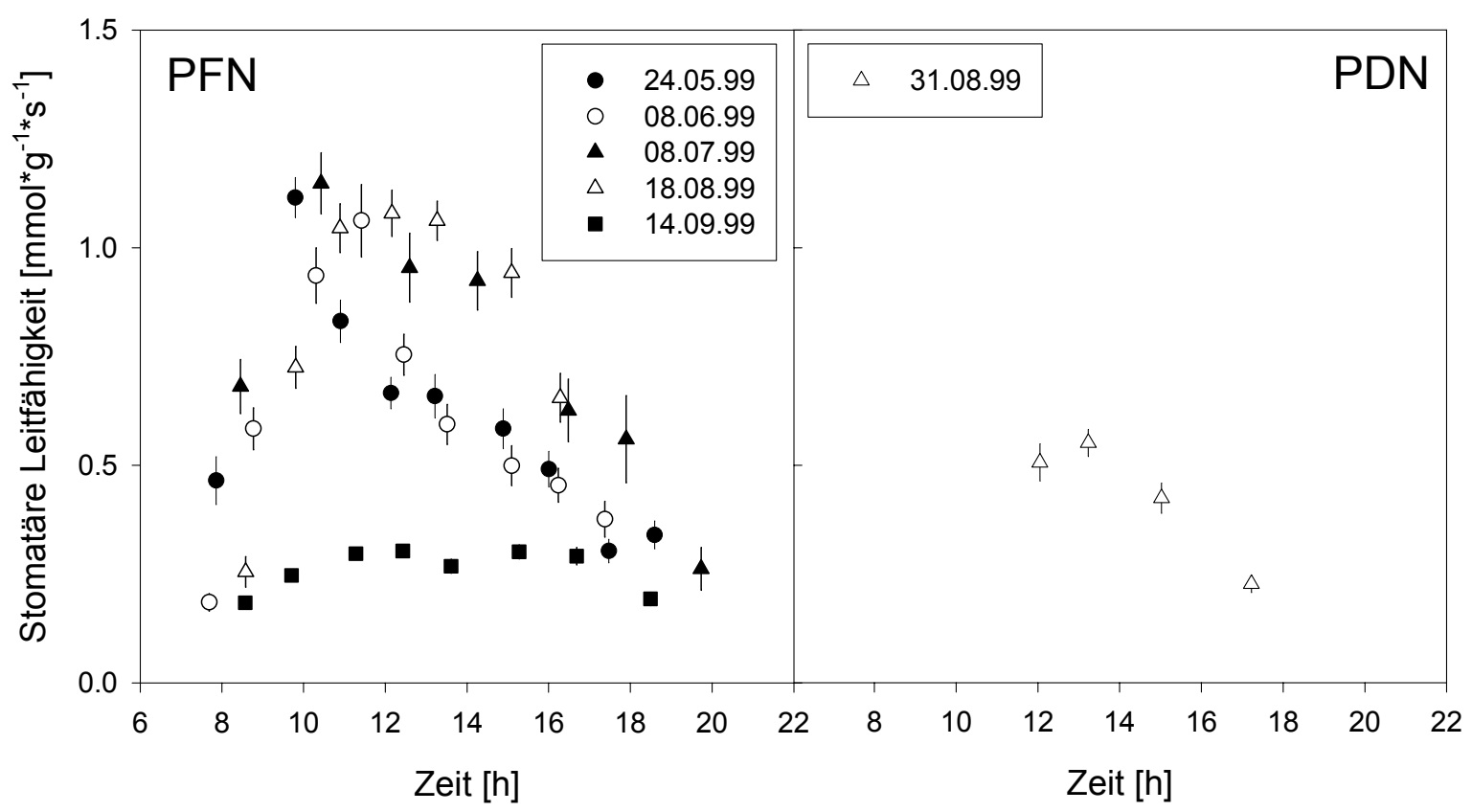

Abb. 4.5.16: Stomatäre Leitfähigkeit von Populus euphratica 2000.

Die trockengewichtsbezogenen Werte wurden parallel auf der im Vorjahr bewässerten ungenutzten (PFN) und genutzten (PFU) Fläche gemessen. 


\subsection{Xylemsaftfluss}

Der Xylemsaftfluss folgte bei allen vier Pflanzenarten der Globalstrahlung. Dabei stieg morgens der Saftfluss eine halbe Stunde (C. caput-medusae, T. ramosissima) bis eine Stunde ( $A$. sparsifolia, $P$. euphratica) später als die Strahlung an und sank am Nachmittag auch wieder mit einer Zeitverzögerung von etwa einer halben Stunde (C. caput-medusae, T. ramosissima) oder einer Stunde ( $A$. sparsifolia, P. euphratica) ab (nicht dargestellt).

Der Saftfluss der untersuchten Arten beschrieb unterschiedliche Tagesverläufe:

A. sparsifolia zeigte einen raschen Anstieg der Saftflussrate am Morgen, die höchsten Werte am frühen Nachmittag und einen wiederum steilen Abfall am Abend. Bei C. caput-medusae und T. ramosissima stieg der Saftfluss morgens steil an und blieb tagsüber auf einem etwa gleichbleibenden Niveau, bis er am Nachmittag wieder abnahm. Bei $P$. euphratica war ein flacherer Anstieg der Saftflusskurve als bei den anderen Arten zu beobachten, auch am Nachmittag sank die Saftflussrate langsamer (nicht dargestellt).

Es war an den meisten Tagen keine Einschränkung des Saftflusses am Mittag zu erkennen, die durch einen Spaltöffnungsschluss (Mittagsdepression der Transpiration) hervorgerufen werden könnte. Nur an wenigen Tagen von Juni bis September 1999 wurden bei einem Sensor auf der Tamarix-Fläche ein Absinken des Saftflusses am Nachmittag beobachtet. Bei $P$. euphratica zeigte ein Baum im Juni 1999 nachmittags eine leichte Reduktion der Saftflussmenge.

Bei Beginn der Messungen im April 1999 wurde jedoch bei T. ramosissima die geringste Saftflussrate im Tagesverlauf mittags gemessen, den Rest des Tages und in der Nacht blieb die Kurve auf einem höheren Niveau. Auch bei C. caputmedusae und A. sparsifolia trat dieses Phänomen im April und Anfang Mai 1999 auf. $\mathrm{Zu}$ dieser Jahreszeit waren die Saftflussraten allerdings noch vergleichsweise niedrig und erreichten erst etwa 10 \% der Sommerwerte. Ende April oder Anfang Mai konnte an den verschiedenen Trieben nacheinander die Umstellung auf einen Tagesverlauf beobachtet werden, der mittags die höchsten und nachts die niedrigsten Werte aufwies. Bei $P$. euphratica wurde nichts Vergleichbares gemessen. Die Pappeln trieben Anfang April 1999 aus, wurden allerdings kurz nach dem Anbringen der Sensoren am 13./14. April von Spannerraupen kahlgefressen und trieben erst Anfang Mai wieder aus. 
Der Einfluss der Tage mit hoher Windgeschwindigkeit und Staubbelastung der Luft waren bei den Saftflussmessungen eindeutig nachzuweisen. An Sturmtagen und bei starker Aufwirbelung von Schluff und Sand vor allem auch an den folgenden Tagen sank der Saftfluss stark ab, da auch die Strahlungssumme sehr gering war. In Folge des starken Windes in der Nacht vom 23. auf den 24. April 1999 sank die trockengewichtsbezogene Saftflusssumme bei C. caput-medusae auf weniger als ein Fünftel des Wertes vor dem Windereignis ab (Tab. 5). Durch die starke Staubund Sandaufwirbelung war die Sonne verdeckt und es kam zu einem jähen Temperatursturz. Die Pflanzen benötigten mehrere Tage, um wieder ähnliche Saftflusswerte wie vorher zu erreichen. Bei einem kürzeren Staubsturm im Juni 2000 sank der Saftfluss ebenfalls stark ab. Weil nicht so viel Staub aufgewirbelt worden war, wurden schneller wieder Bedingungen wie zuvor hergestellt.

Tab. 5: Saftflusssummen von Calligonum caput-medusae und Witterungsbedingungen bei Sandstürmen 1999 und 2000.

Die Mittelwerte der Saftflusssummen von 6 Sensoren an Tagen mit hohen Windgeschwindigkeiten; zum Vergleich die Tage vor und nach den Sandstürmen.

\begin{tabular}{c|c|c|cc|c|c}
\hline Datum & $\begin{array}{c}\text { Mittl. Luft- } \\
\text { temperatur } \\
{\left[{ }^{\circ} \mathrm{C}\right]}\end{array}$ & $\begin{array}{c}\text { Mittl. } \\
\text { VPD } \\
{[\mathrm{kPa}]}\end{array}$ & $\begin{array}{c}\text { Windgeschwindig- } \\
\text { keit }\left[\mathrm{m}^{*} \mathrm{~s}^{-1}\right]\end{array}$ & $\begin{array}{c}\text { Summe } \\
\text { Mittlere }\end{array}$ & $\begin{array}{c}\text { Maximale } \\
\text { Globalstrahlung } \\
{\left[\mathrm{W}^{*} \mathrm{~m}^{-2 *} \mathrm{~d}^{-1}\right]}\end{array}$ & $\begin{array}{c}\text { Summe } \\
\text { Saftfluss }\end{array}$ \\
\hline 22.4 .99 & 22.98 & 2.66 & 2.51 & 5.27 & 36842.67 & $38.95 \pm 5.41$ \\
23.4 .99 & 20.89 & 2.10 & 6.69 & 10.93 & 29148.05 & $30.09 \pm 4.98$ \\
24.4 .99 & 14.00 & 1.32 & 6.84 & 10.56 & 16734.20 & $15.93 \pm 2.36$ \\
25.4 .99 & 5.49 & 0.46 & 2.13 & 4.31 & 10058.53 & $6.98 \pm 0.73$ \\
26.4 .99 & 9.11 & 0.78 & 1.58 & 2.77 & 15713.95 & $7.55 \pm 0.46$ \\
27.4 .99 & 12.68 & 1.08 & 1.41 & 3.36 & 21082.19 & $8.70 \pm 0.67$ \\
28.4 .99 & 14.43 & 1.32 & 1.11 & 2.87 & 24585.99 & $9.45 \pm 0.93$ \\
29.4 .99 & 17.69 & 1.77 & 1.96 & 4.16 & 28573.20 & $12.74 \pm 1.49$ \\
\hline 20.6 .00 & 27.71 & 2.99 & 1.57 & 3.45 & 38658.40 & $10.33 \pm 1.99$ \\
21.6 .00 & 28.36 & 3.19 & 1.65 & 4.04 & 40092.06 & $10.39 \pm 2.03$ \\
22.6 .00 & 25.33 & 2.14 & 6.50 & 10.67 & 13539.14 & $6.36 \pm 1.23$ \\
23.6 .00 & 20.14 & 0.94 & 2.41 & 7.78 & 30343.45 & $8.38 \pm 1.72$ \\
24.6 .00 & 21.77 & 1.42 & 2.29 & 5.44 & 50312.87 & $11.11 \pm 2.28$ \\
\hline
\end{tabular}

Vergleicht man die über die Vegetationsperiode gemittelten Saftflussraten der Arten untereinander, so zeigt die $C_{4}$-Pflanze $C$. caput-medusae mit 12,3 $\pm 1,6 \mathrm{~g}$ Wasser pro g Blatttrockengewicht und Tag $\left[\mathrm{g} \mathrm{H}_{2} \mathrm{O}^{*} \mathrm{~g} \mathrm{TG}^{-1 *} \mathrm{~d}^{-1}\right]$ sehr niedrige Werte. Alhagi sparsifolia hat eine mittlere Saftflussrate von 14,45 $\pm 1,2 \mathrm{~g} \mathrm{H}_{2} \mathrm{O}^{*} \mathrm{~g} \mathrm{TG}^{-1 *} \mathrm{~d}^{-1}$, während Tamarix ramosissima mit 14,8 $\pm 1,75 \mathrm{~g} \mathrm{H}_{2} \mathrm{O}^{*} \mathrm{~g} \mathrm{TG}^{-1 *} \mathrm{~d}^{-1}$ nur wenig mehr Wasser verbraucht. Es ist erstaunlich, dass die Arten mit reduzierten Blättern wie der Rutenstrauch $C$. caput-medusae und $T$. ramosissima an diesem Standort 
genauso viel Wasser verbrauchen wie $A$. sparsifolia als Art mit flächig ausgebildeten Blättern.

Die geringsten auf das Blatttrockengewicht bezogenen Saftflussraten wurden bei Populus euphratica mit nur 2,1 $\pm 0,4 \mathrm{~g} \mathrm{H}_{2} \mathrm{O}^{*} \mathrm{~g} \mathrm{TG}^{-1 *} \mathrm{~d}^{-1}$ gemessen. Diese sehr niedrigen Werte bei $P$. euphratica sind allerdings auf Messfehler zurückzuführen (siehe Kapitel 4.6.2) und daher nicht glaubwürdig.

\subsubsection{Modellierung des Saftflusses 1999}

\section{Modellierung für das System nach KUČERA am Beispiel von Alhagi sparsifolia}

Bei $A$. sparsifolia traten sehr hohe Saftflussraten auf, was dazu führte, dass die in den Logger integrierte Heizung zur Mittagszeit, wenn der Saftfluss am höchsten war, nicht die erforderliche Heizleistung erbrachte. Dies fällt zunächst bei denjenigen der sechs Sensoren auf, die die höchsten Saftflussraten aufwiesen. Bei diesen Sensoren nämlich kam es schon Anfang Juni zu mehrstündigen Messlücken während der Mittagszeit (Abb. 3.7.1). Die anderen Sensoren folgten etwas später im Jahr diesem Muster, nur die Sensoren 1 und 6, die relativ geringe Saftflussleistung anzeigten, wurden nicht dadurch beeinträchtigt. Sie liefen, sofern es keine Messausfälle anderer Art gab, während der gesamten Vegetationsperiode.

Deswegen wurde der Sensor 1 als Berechnungsgrundlage für die Modellierung der fehlenden Daten ausgewählt. Abb. 4.6.1 zeigt beispielhaft das Verhältnis der anderen Sensoren zu Sensor 1. Die Beziehungen zwischen den Sensoren wurden für verschiedene Jahreszeiten (Frühsommer, Sommer, Spätsommer) berechnet und waren immer sehr eng.

Für die kompletten Messlücken sollte der Saftfluss aus Klimaparametern berechnet werden. Das Verhältnis der Saftflussrate von Sensor 1 zur an der Klimastation Qira 1 gemessenen Globalstrahlung ließ sich durch einen exponentiellen Anstieg zum Maximum beschreiben (Abb. 4.6.2). Vergleicht man die gemessenen Saftflussraten mit den aus dieser Beziehung berechneten Werten, erkennt man nur geringe Abweichungen. Die aufgrund der Strahlung berechneten Saftflussraten zeigten 
nachts einen fast konstanten Wert. Die tatsächlich gemessenen Saftflussraten wiesen jedoch nächtliche Schwankungen auf. Dieser nächtliche Saftfluss schien manchmal der Lufttemperatur zu folgen, ließ sich aber in keine klare Beziehung fassen.
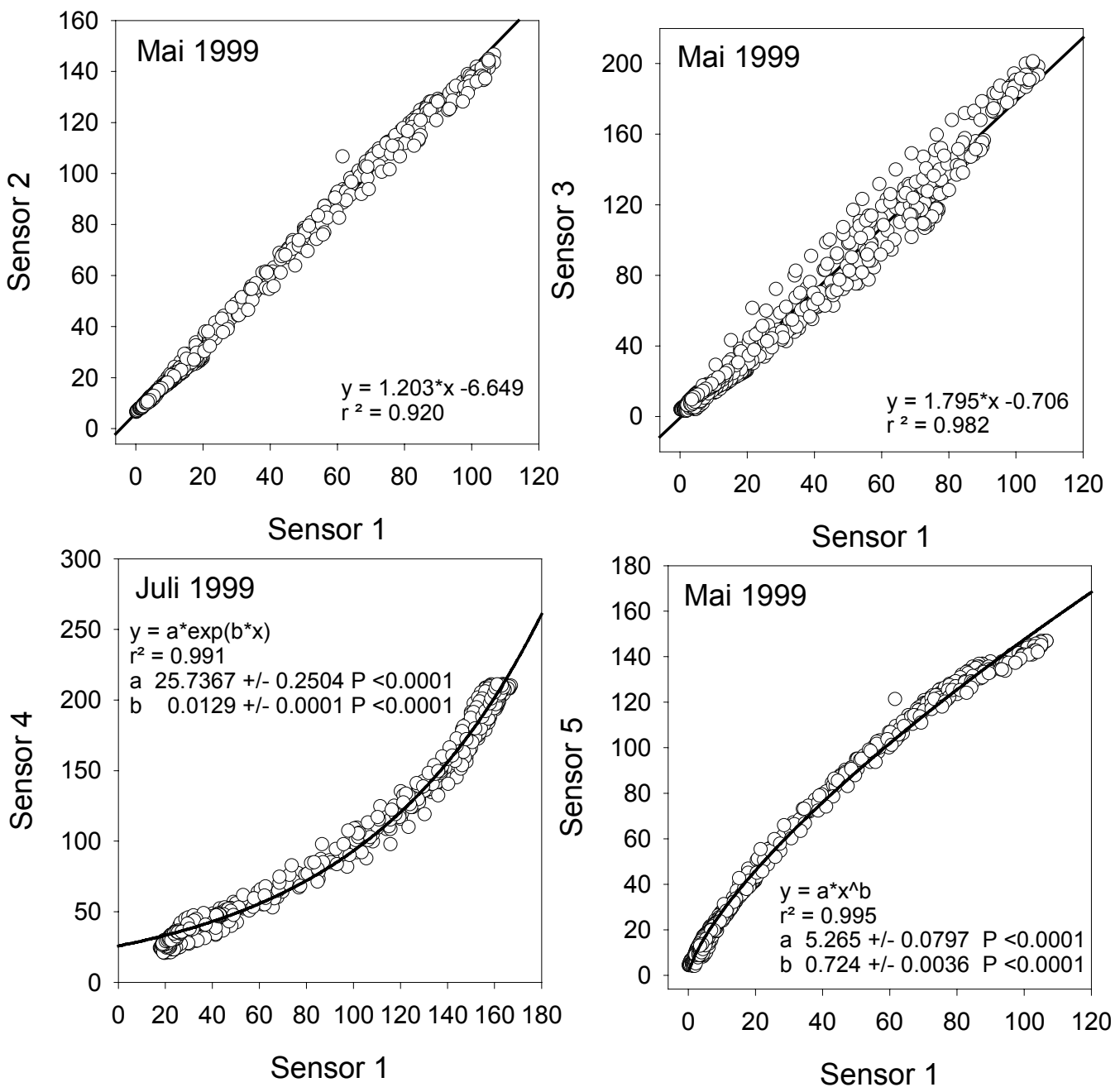

Abb. 4.6.1: Verhältnisse der Saftflussraten der Sensoren 2-5 vs. Sensor 1 am Beispiel von Alhagi sparsifolia.

Beispielhaft wurden die Verhältnisse der Saftflussraten $\left[g^{*} h^{-1}\right]$ der Sensoren in den Monaten Mai bzw. Juli 1999 ausgewählt. Regressionsgleichungen und Irrtumswahrscheinlichkeit sind angegeben. 


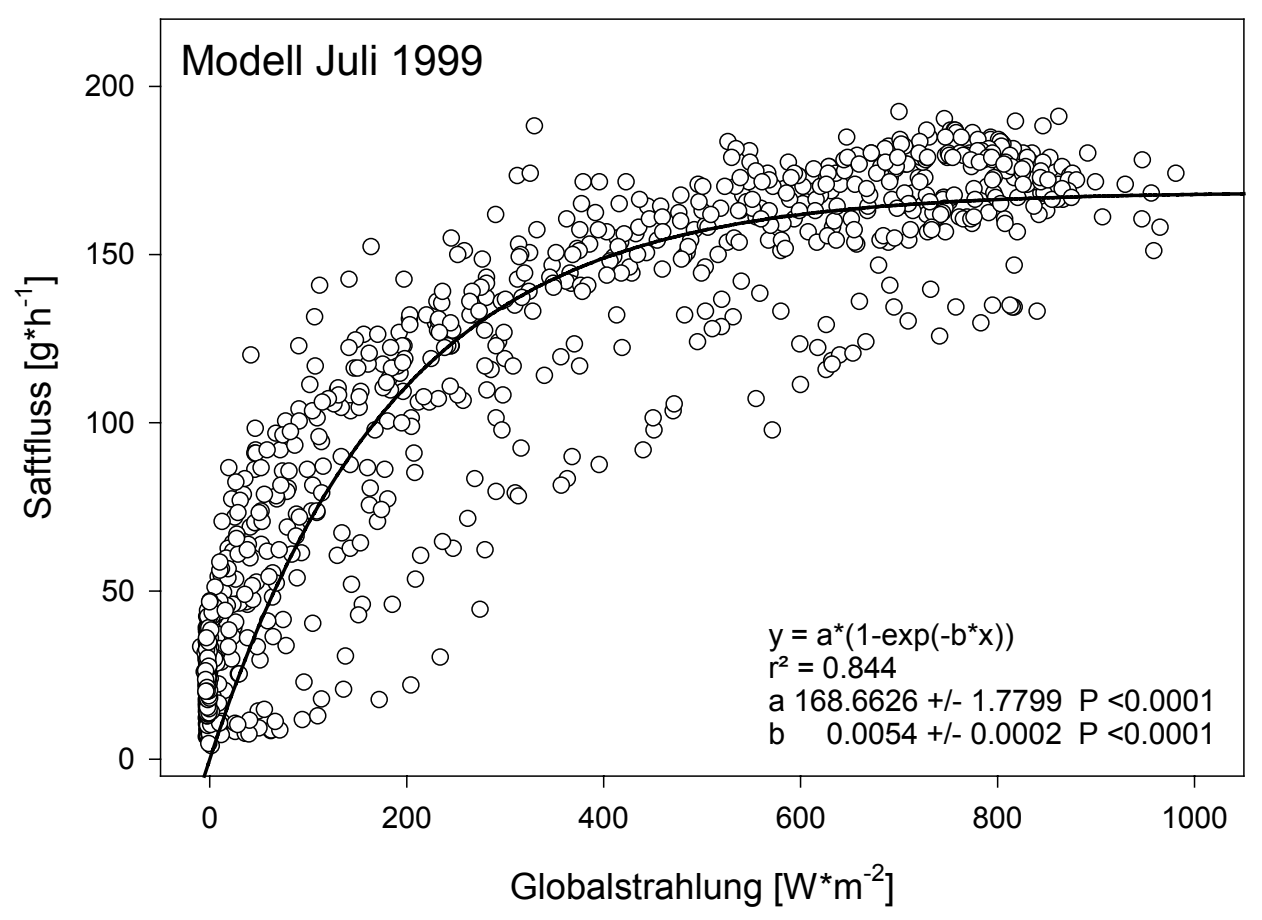

Abb. 4.6.2: Globalstrahlung vs. Saftflussrate des Sensors 1 von Alhagi sparsifolia im Juli 1999.

Die Werte der Globalstrahlung wurden von der Wetterstation Qira_1 auf der Alhagi-Fläche aufgezeichnet. Die Regressionsgleichung ist mit Regressionskoeffizient und Irrtumswahrscheinlichkeit dargestellt.

An den meisten Tagen wurde jedoch gar kein Modell für die nächtlichen Saftflussraten benötigt, da die Sensoren vom späten Nachmittag oder Abend bis zum Morgen des folgenden Tages liefen und nur tagsüber ausfielen. Trotz der Unterschiede zwischen Modell und Messung nachts wurde das Modell auch für Tage verwendet, an denen die Logger komplett ausgefallen waren. Wenn man die gemessenen Tagessummen des Saftflusses mit den modellierten Tagessummen vergleicht, stellt man fest, dass die nächtlichen Unterschiede zwischen Modell und Messung kaum ins Gewicht fallen (nicht dargestellt). Zwischen den beiden Tagessummen besteht nur ein geringer Unterschied.

Für die Sensoren war das Verhältnis zu Sensor 1 an manchen Tagen enger korreliert als zur Strahlung. In diesem Fall wurde die Saftflussrate über die Beziehung zum laufenden Sensor berechnet. Es wurde für jede Messlücke verglichen, mit welcher Methode sich zu einer gegebenen Jahreszeit die Saftflussrate genauer 
berechnen ließ. Dazu wurden möglichst die Tage vor und nach der zu schließenden Messlücke zum Vergleich herangezogen.

Wenn man nur die gemessenen Tagessummen von Tagen ohne Messlücken heranzieht, ergibt sich ein sehr lückenhaftes Bild (Abb. 4.6.3 A). Die leeren Bereiche sind Tage, an denen keine Daten aufgezeichnet wurden, die grau unterlegten Bereiche kennzeichnen Tage mit kleineren Messlücken um die Mittagszeit, an denen auch die nicht dargestellten Sensoren Daten lieferten, jedoch keine vollständigen Tagesgänge. In Abb. 4.6.3 B sind die vollständig modellierten Werte dargestellt. Das Modell unterschätzt die Saftflusswerte um 3,1 \% (Juni) bis maximal 13,3 \% (August).

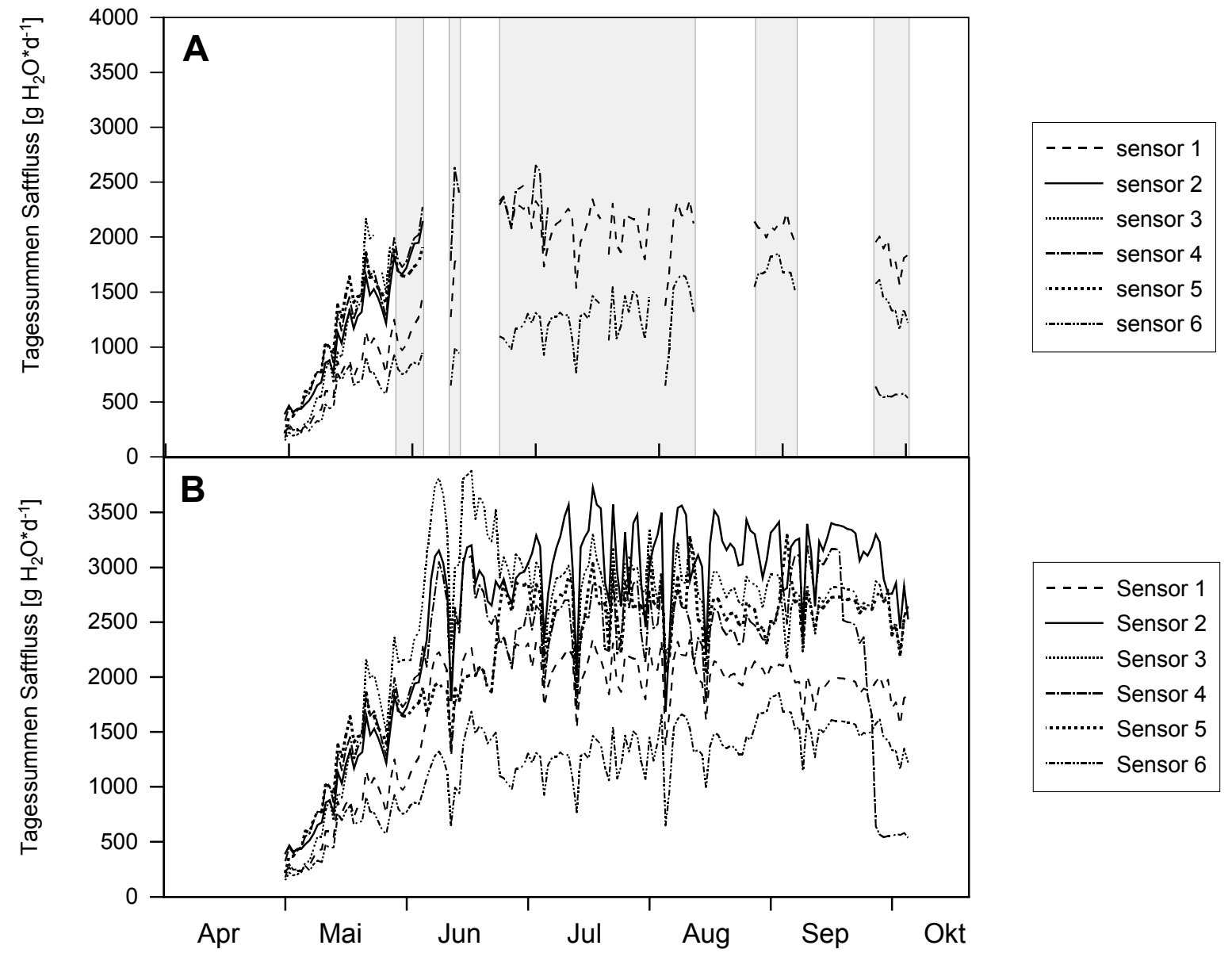

Abb. 4.6.3: Gemessene und modellierte Tagessummen des Saftflusses von Alhagi sparsifolia 1999.

Saftflusstagessummen von 6 Sensoren auf der bewässerten ungenutzten Fläche (AFN). A: Die leeren Bereiche kennzeichnen vollständigen Messausfall, die grau unterlegten Bereiche Tage mit kleineren Messlücken, für die jedoch keine Tagessumme berechnet werden konnte. B: Gemessene Werte ergänzt mit modellierten Werten. 
Ebenso wurden die fehlenden Daten für C. caput-medusae und T. ramosissima modelliert.

Bei C. caput-medusae standen die Sensoren 1 bis 4 während der gesamten Vegetationsperiode zur Verfügung, die Sensoren 5 und 6 fehlten ab dem 1. August 1999, da Ende Juli einige Sensoren gestohlen wurden. Modelliert wurde mit den Werten von Sensor 2. Die mit der Globalstrahlung modellierten Werte unterschätzen die gemessenen Werte um 6,6 \% (Mai). Die Abweichung in den anderen Monaten ist eine Überschätzung von 3,7 \% bis knapp $5 \%$.

Bei T. ramosissima gab es nur Ausfälle bei den Sensoren 3, 4 und 5. Modelliert wurde mit den Werten von Sensor 1. Die Abweichung zwischen Messung und Modell besteht in einer Unterschätzung durch das Modell von 4,5-5 \% von Mai bis Juli. Im Spätsommer liegen Modell und Messung noch näher zusammen.

Vergleicht man die modellierten Saftflussmengen (als g Wasser ${ }^{*}$ Sensor $^{-1}{ }^{*} \mathrm{Tag}^{-1}$ ) der vier untersuchten Arten, ist zwischen den Sensoren eine große Variabilität zu erkennen (beispielhaft für $A$. sparsifolia in Abb. 4.6.3 dargestellt). Trägt man die Tagessummen des Saftflusses auf das Trockengewicht bezogen auf, wird die Variabilität geringer. Dann zeigen vor allem $A$. sparsifolia und C. caput-medusae im April und Anfang Mai viel höhere Saftflussraten pro Gramm Trockengewicht als im weiteren Verlauf des Jahres. Auch $P$. euphratica zeigt höheren Saftfluss im Frühjahr. Die Saftflussrate pendelt sich ab Ende Mai bei diesen Arten auf ein im Rest der Vegetationsperiode gleichbleibendes Niveau ein:

Alhagi sparsifolia 9,3 - 15,3 $\mathrm{g} \mathrm{H}_{2} \mathrm{O}^{*} \mathrm{~g} \mathrm{TG}^{-1 *} \mathrm{~d}^{-1}$ (Mittelwert 12,6 $\mathrm{g} \mathrm{H}_{2} \mathrm{O}^{*} \mathrm{~g} \mathrm{TG}^{-1 *} \mathrm{~d}^{-1}$ ), Calligonum caput-medusae 5,5 - 17,5 $\mathrm{g} \mathrm{H}_{2} \mathrm{O}^{*} \mathrm{~g} \mathrm{TG}^{-1 *} \mathrm{~d}^{-1}\left(9,3 \mathrm{~g} \mathrm{H}_{2} \mathrm{O}^{*} \mathrm{~g} \mathrm{TG}^{-1 *} \mathrm{~d}^{-1}\right.$ als Mittelwert). Bei Populus euphratica liegt der Wert mit $0,3-3,4 \mathrm{~g} \mathrm{H}_{2} \mathrm{O}^{*} \mathrm{~g} \mathrm{TG}^{-1 *} \mathrm{~d}^{-1}$ (Mittelwert 1,39 $\mathrm{g} \mathrm{H}_{2} \mathrm{O}^{*} \mathrm{~g} \mathrm{TG}^{-1 *} \mathrm{~d}^{-1}$ ) sehr niedrig und ist nicht glaubwürdig, da es bei dieser Art Probleme mit dem Saftflusssystem gab (siehe auch Kapitel 5.4.2).

T. ramosissima zeigt dagegen im Frühling sehr niedrige Werte und die trockengewichtsbezogene Saftflussrate bleibt über das ganze Jahr auf einer konstanten Höhe von 10,2 - 22,2 $\mathrm{g} \mathrm{H}_{2} \mathrm{O}^{*} \mathrm{~g} \mathrm{TG}^{-1 *} \mathrm{~d}^{-1}$ (Mittelwert 16,1 $\mathrm{g} \mathrm{H}_{2} \mathrm{O}^{*} \mathrm{~g} \mathrm{TG}{ }^{-1 *} \mathrm{~d}^{-1}$ ). 

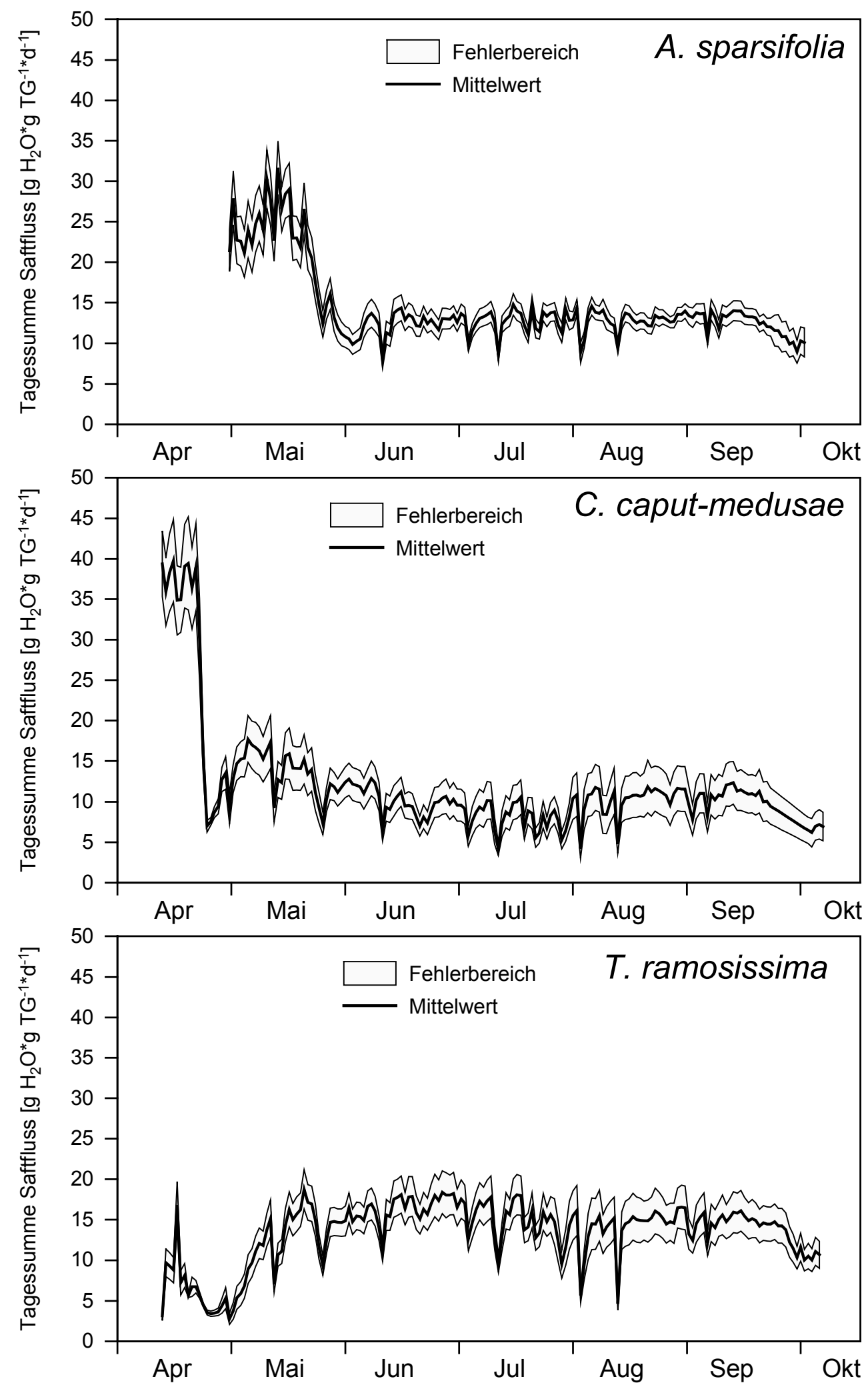

Abb. 4.6.4: Modellierte Tagessummen des Saftflusses von Alhagi sparsifolia, Calligonum caput-medusae und Tamarix ramosissima 1999.

Die Tagessummen sind als Mittelwerte von jeweils 6 Sensoren (Alhagi sparsifolia und Tamarix ramosissima) mit Standardfehlern dargestellt. Bei Calligonum caput-medusae sind ab August 1999 nur noch 4 Sensoren vorhanden. Die Werte sind auf das Blatttrockengewicht bezogen. 


\section{Modellierung für das System nach GRANIER}

Für das GRANIER-Saftfluss-System wurde ein anderer Weg der Modellierung gewählt. Die Globalstrahlung korrelierte nicht eng genug mit den gemessenen Saftflussdichten, engere Beziehungen konnten mit dem VPD berechnet werden. An manchen Tagen konnten kleinere Messausfälle auch mit dem Verhältnis zum laufenden Sensor nachberechnet werden. Nicht alle Messlücken 1999 konnten mit dieser Modellierung geschlossen werden: im Frühjahr lieferten nur zwei von drei laufenden Sensoren ausreichend Messwerte, drei weitere Sensoren konnten wegen einer defekten Heizanlage nicht betrieben werden. Im Sommer kam es zu einem etwa sechswöchigen Ausfall der gesamten Anlage. Ab Ende Juli liefen alle sechs Sensoren. Man kann sehen, dass die beiden Bäume, an denen im Frühjahr gemessen wurde, sehr geringe Saftflussraten im Vergleich zu den anderen untersuchten Bäumen aufweisen (Abb. 4.6.5).

Die Modellierung unterscheidet sich von der Messung durch eine Unterschätzung von ungefähr $3 \%$ im Frühsommer und durch eine Überschätzung um ca. 3-5 \% im Spätsommer.

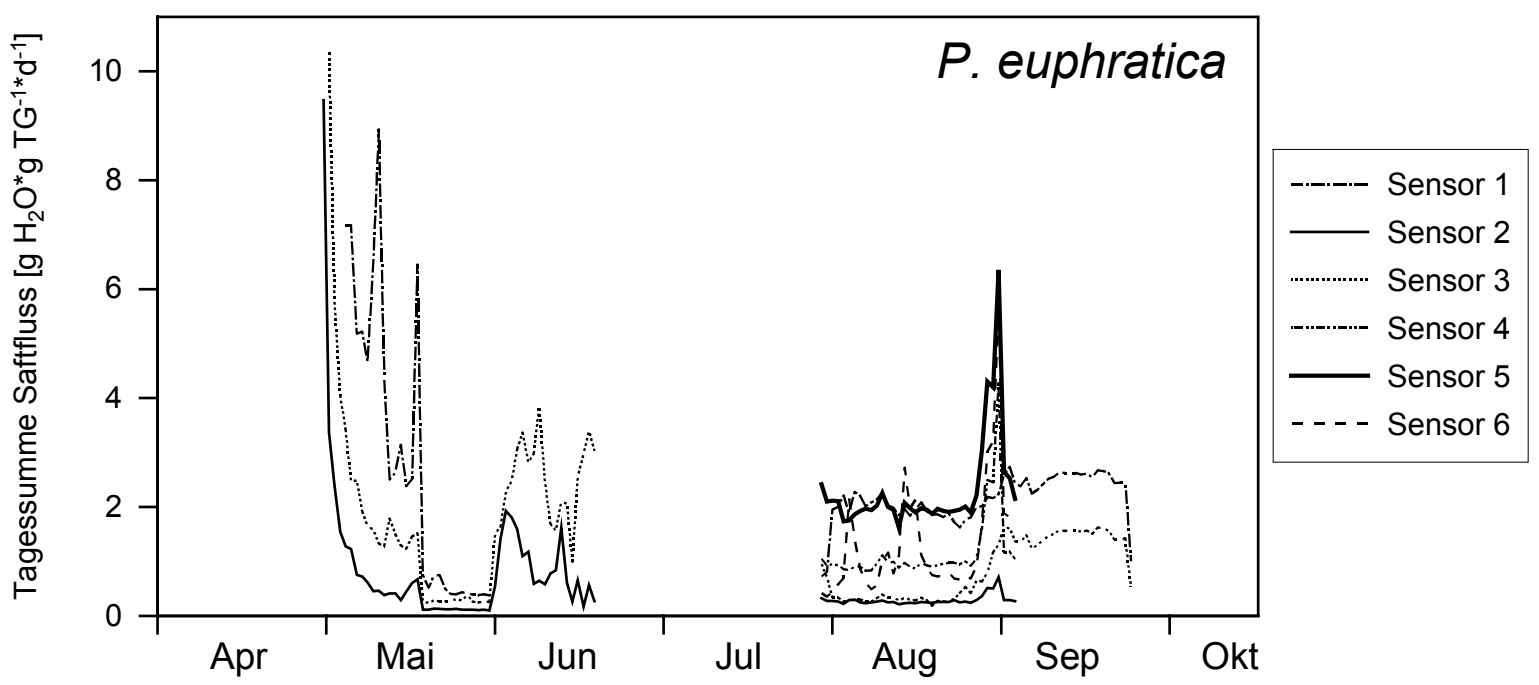

Abb. 4.6.5: Modellierte Tagessummen des Saftflusses von Populus euphratica 1999.

Die trockengewichtsbezogenen Tagessummen der jeweils laufenden Sensoren sind dargestellt. Kleinere Messlücken sind durch Modellierung geschlossen. 


\section{Effekt der Bewässerung auf den Saftfluss}

Ob sich der Saftfluss der untersuchten Arten vor und nach der Überflutung unterscheidet, wurde statistisch untersucht. Dazu wurde für jede Art je ein Tag vor und nach der Überflutung ausgewählt, an dem sich die klimatischen Parameter möglichst ähnlich sind. Geprüft wurde vor allem die Strahlungssumme der Tage und das VPD, aber auch Lufttemperatur und Luftfeuchte wurden bei der Auswahl der zu vergleichenden Tage berücksichtigt (Tab. 11 im Anhang). Die Saftflusssummen vor der Überflutung unterscheiden sich nicht signifikant von denen nach der Überflutung.

\subsubsection{Saftflussmessungen und Modellierung 2000}

Auch im Jahr 2000 wurden bei A. sparsifolia und C. caput-medusae im Frühjahr wieder höhere trockengewichtsbezogene Saftflussraten gemessen als im Frühsommer (Abb. 4.6.6). Die Saftflussraten erreichten bei diesen Arten ähnliche Werte wie im Vorjahr. Anders als 1999 zeigte auch Tamarix ramosissima im Mai 2000 mit über $70 \mathrm{~g} \mathrm{H}_{2} \mathrm{O}^{*} \mathrm{~g} \mathrm{TG}^{-1 *} \mathrm{~d}^{-1}$ sehr hohe Werte.

Im Juli 2000 fielen aufgrund technischer Probleme nacheinander die Datalogger des Saftflusssystems nach KUČERA aus. Im Frühsommer zeichneten die Logger noch Daten auf, waren aber schon nicht mehr voll funktionstüchtig. Dies wird am Beispiel von Tamarix ramosissima in Abb. 4.6.7 illustriert: dargestellt sind die Saftflussraten und die klimatischen Bedingungen vom 1.-8. Juni 2000. Bei einigen der Sensoren war plötzlich ein Absinken des Saftflusses während der Mittagsstunden zu erkennen (vor allem bei Sensor 1, 3 und 4), während die Sensoren 5 und 6 zwar sehr viel geringere Saftflussraten aufwiesen, aber nach wie vor den maximalen Saftfluss mittags zeigten. Obwohl sich die Witterung nicht unterschied, änderten sich also die Saftflussraten und der Verlauf der Tagesgänge schlagartig. Dies traf zeitgleich mit zwei voneinander unabhängigen Phänomenen ein: zum einen traten in den ersten Junitagen die ersten technischen Probleme am Logger auf, zum anderen wurde $T$. ramosissima von bisher nicht identifizierten Käferlarven kahl gefressen. Daher sanken auch die Saftflussraten der funktionierenden Sensoren ab. Es konnte nicht verfolgt werden, ob und wann der Saftfluss an den intakten Sensoren völlig zum Erliegen kam, da für einige Wochen keine Daten 
vorliegen. Ab Ende Juni zeichnete der Logger wieder Daten auf. Man kann ein Ansteigen der Saftflussrate erkennen, das zeitgleich mit einem erneuten Austreiben der Tamarisken stattfand.

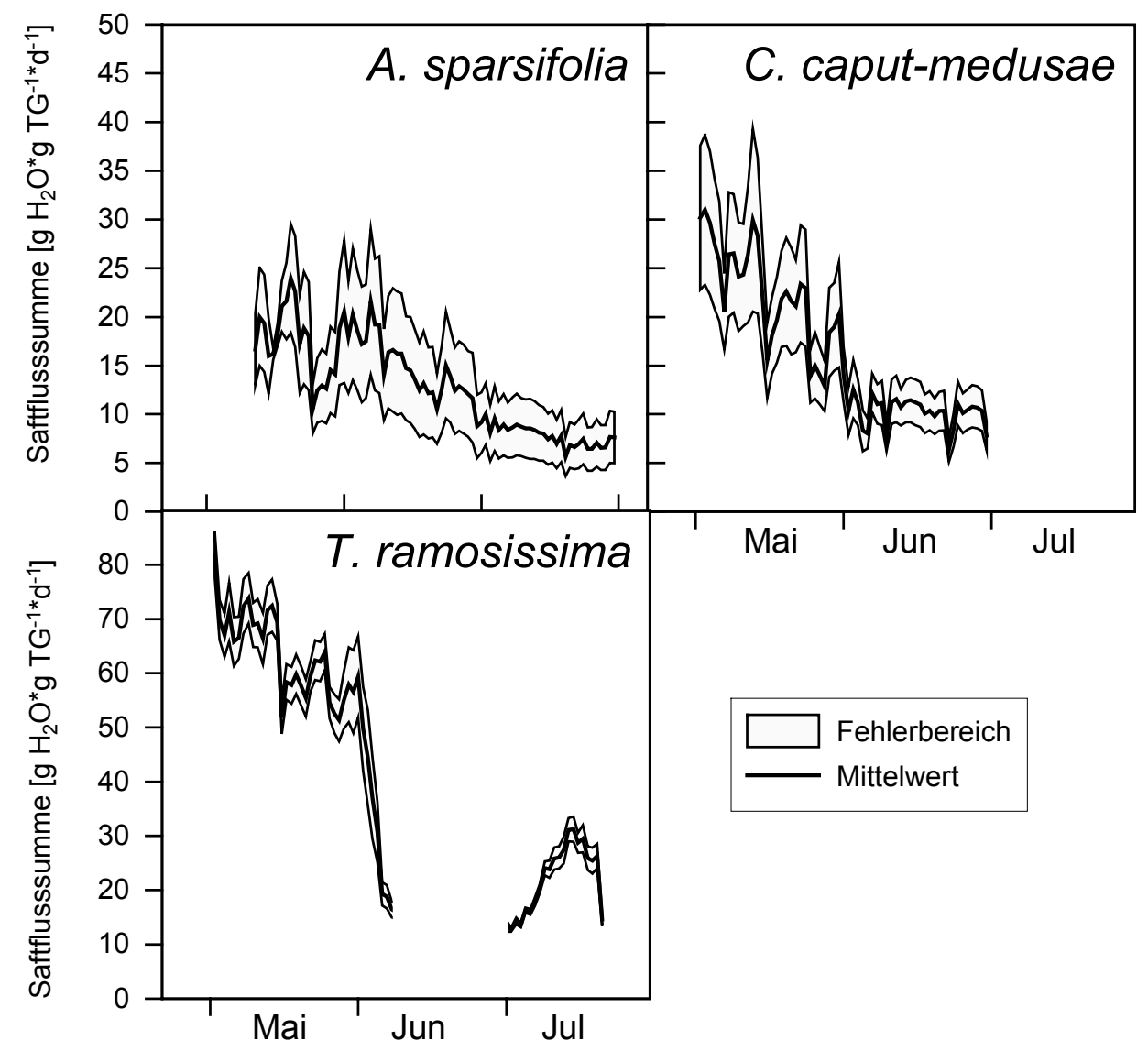

Abb. 4.6.6: Modellierte Tagessummen des Saftflusses von Alhagi sparsifolia, Calligonum caput-medusae und Tamarix ramosissima 2000.

Dargestellt sind als trockengewichtsbezogene Tagessumme nur der Mittelwert der funktionierenden Sensoren. Die Lücke bei $T$. ramosissima entstand durch Kahlfrass der Pflanzen.

Der Logger auf der TFN-Fläche fiel im Juli 2000 endgültig aus. Daher konnte der Saftfluss während der vollständigen Wiederbegrünung von T. ramosissima nicht gemessen werden. Der Saftfluss während des erneuten Kahlfrasses Ende Juli bis Anfang August und im Verlauf des darauf folgenden Austreibens Ende August konnte ebenfalls nicht aufgezeichnet werden. 


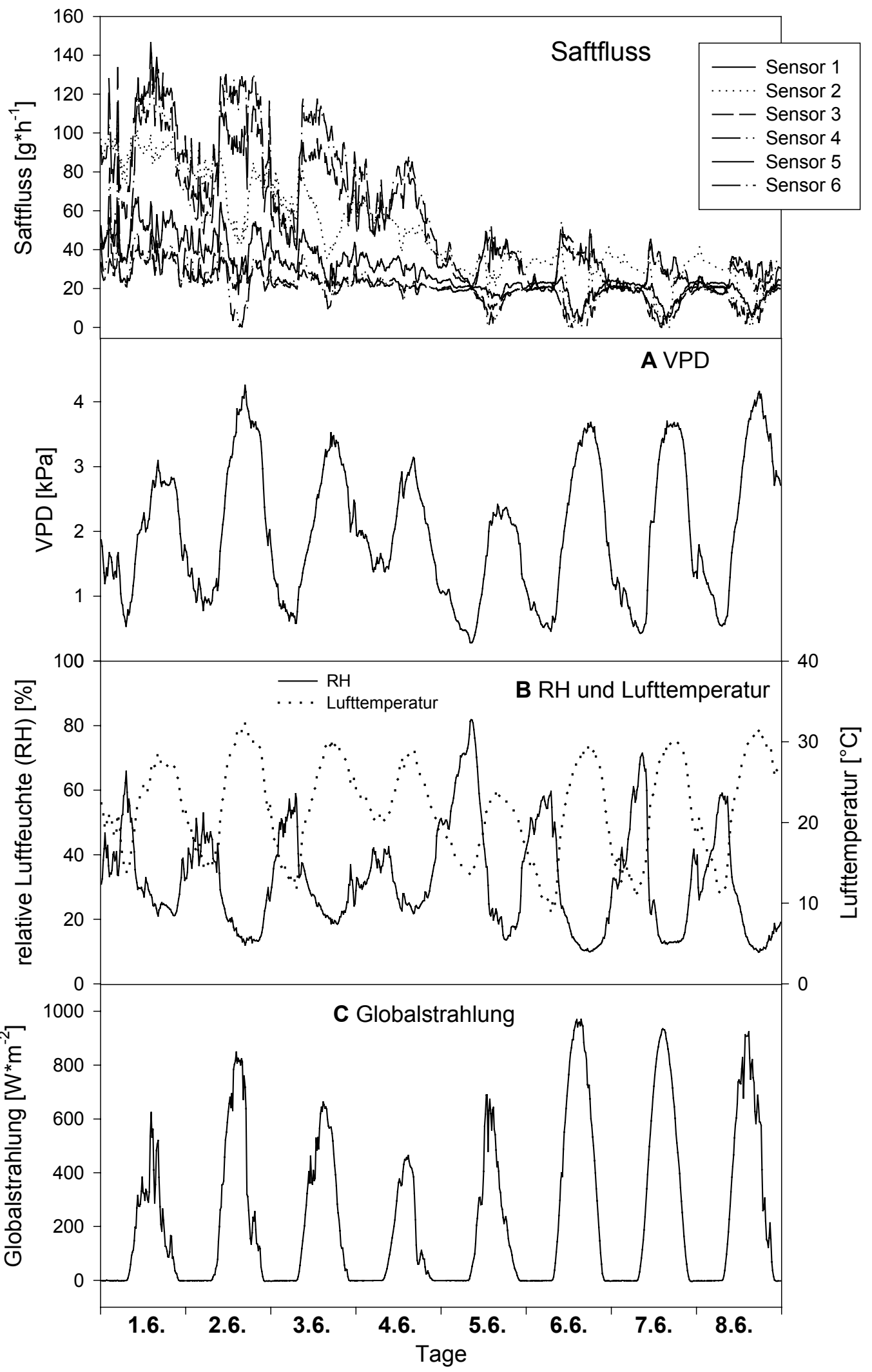

Abb. 4.6.7: Tamarix ramosissima: Saftflussrate und Witterungsbedingungen vom 1.-8.6.2000.

Dargestellt ist der von 6 Sensoren gemessene Saftfluss sowie das VPD (A), die relative Luftfeuchte und Lufttemperatur (B) und die Globalstrahlung (C). 
Auch bei A. sparsifolia und C. caput-medusae traten 2000 bei einigen der Sensoren plötzlich veränderte Saftflussraten auf, die sich meist in einer Reduzierung des Saftflusses gegen Null mit anschließendem Ausfall der Geräte am Mittag darstellten (ohne Abbildung).

Bei $A$. sparsifolia fällt auf, dass drei der Pflanzen, an denen Saftfluss gemessen wurde, wie in den Wochen zuvor ihre maximale Saftflussrate mittags hatten. Zwei Pflanzen dagegen zeigten eine rasche Abnahme und anschließend komplette Ausfälle am Nachmittag, die vermutlich auf die erwähnten technischen Probleme am Logger zurückzuführen waren. Auch C. caput-medusae zeigte dieses Phänomen. Daher konnten in der Vegetationsperiode 2000 bei den genannten Pflanzenarten nicht alle 6 Sensoren ausgewertet werden. So weit es möglich war, wurde der Saftfluss für die entstandenen Lücken über das Verhältnis der Sensoren untereinander modelliert. In Abb. 4.6.6 sind nur die Daten der funktionierenden Sensoren dargestellt.

Auf der Populus-Fläche konnte im Jahr 2000 nur mit zwei Sensoren gemessen werden, da die anderen Sensoren kurz nach der Installation zerstört worden waren. Die beiden Sensoren zeigten regelmäßig mehrstündige Messlücken am Vormittag. An einigen vollständigen Messtagen aus verschiedenen Monaten wurden Regressionsgleichungen des VPD gegen Saftflussraten am Vormittag berechnet und mit diesen Beziehungen die fehlenden Werte modelliert.

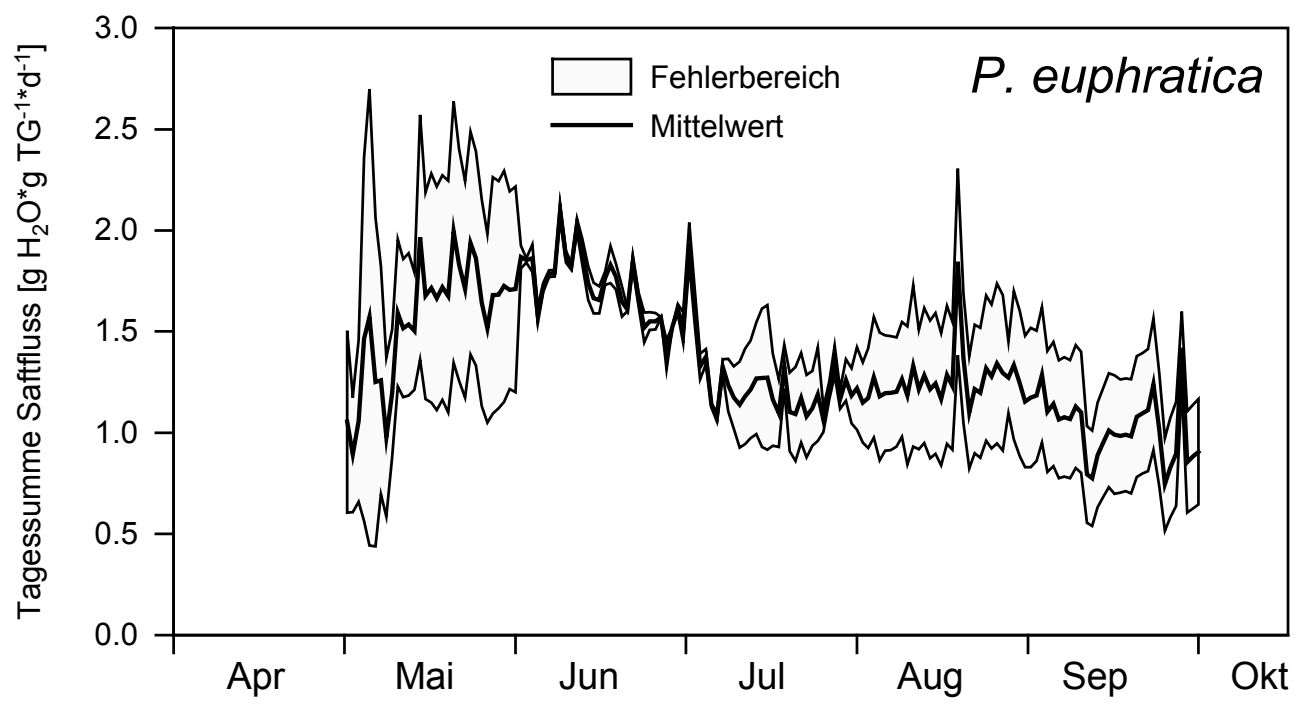

Abb. 4.6.8: Saftflusstagessummen von Populus euphratica 2000.

Modellierte trockengewichtsbezogene Werte der zwei laufenden Sensoren. 
Die modellierten Werte überschätzen den gemessenen Saftfluss im Schnitt um 1-3 \%. Die Saftflusswerte bei $P$. euphratica liegen wie schon 1999 auf einem sehr niedrigen Niveau (Abb. 4.6.8) und müssen als fehlerhafte Werte angesehen werden (siehe Kapitel 5.4.2).

\subsubsection{Vergleich des aus Saftfluss- und Transpirationsmessungen ermittelten Wasserverbrauchs}

Von den trockengewichtsbezogenen Transpirationsmessungen wurde der Wasserverbrauch der Pflanzen durch Interpolation zwischen den Werten sowie zwischen Sonnenaufgang und Beginn der Messung bzw. Ende der Messung und Sonnenuntergang auf die gesamte Lichtperiode des Tages hochgerechnet. Für die Tage, an denen die Transpirationsmessungen stattfanden, wurde zum Vergleich die auf das Blatttrockengewicht bezogene Summe des Saftflusses während des gleichen Zeitraumes berechnet.

Bei der ersten Messkampagne im Mai 1999 wurde bei A. sparsifolia bei der Transpirationsmessung ein höherer Wasserverbrauch als beim Saftfluss festgestellt (Abb. 4.6.9). Bei T. ramosissima waren die berechneten Wassermengen fast gleich. Für C. caput-medusae konnte wegen der fehlenden Transpirationsmessung im Mai 1999 der Wasserverbrauch nicht verglichen werden. Im weiteren Verlauf des Jahres waren die Unterschiede zwischen den beiden Messmethoden bei $A$. sparsifolia sehr variabel, bei $C$. caput-medusae und $T$. ramosissima stellte sich ein relativ konstanter Unterschied ein. Während bei $A$. sparsifolia, C. caputmedusae und $T$. ramosissima an den meisten Terminen bei den Saftflussmessungen höhere Werte als bei den Transpirationsmessungen erreicht wurden, liegen die Saftflusssummen bei $P$. euphratica deutlich unter dem aus Transpirationsmessungen berechneten Wasserverbrauch. Im Gegensatz zur Saftflussmenge, die sich bei $P$. euphratica über die Vegetationsperiode kaum ändert, steigt die Transpirationsmenge leicht an und fält am letzten Messtermin im September 1999 ab. 


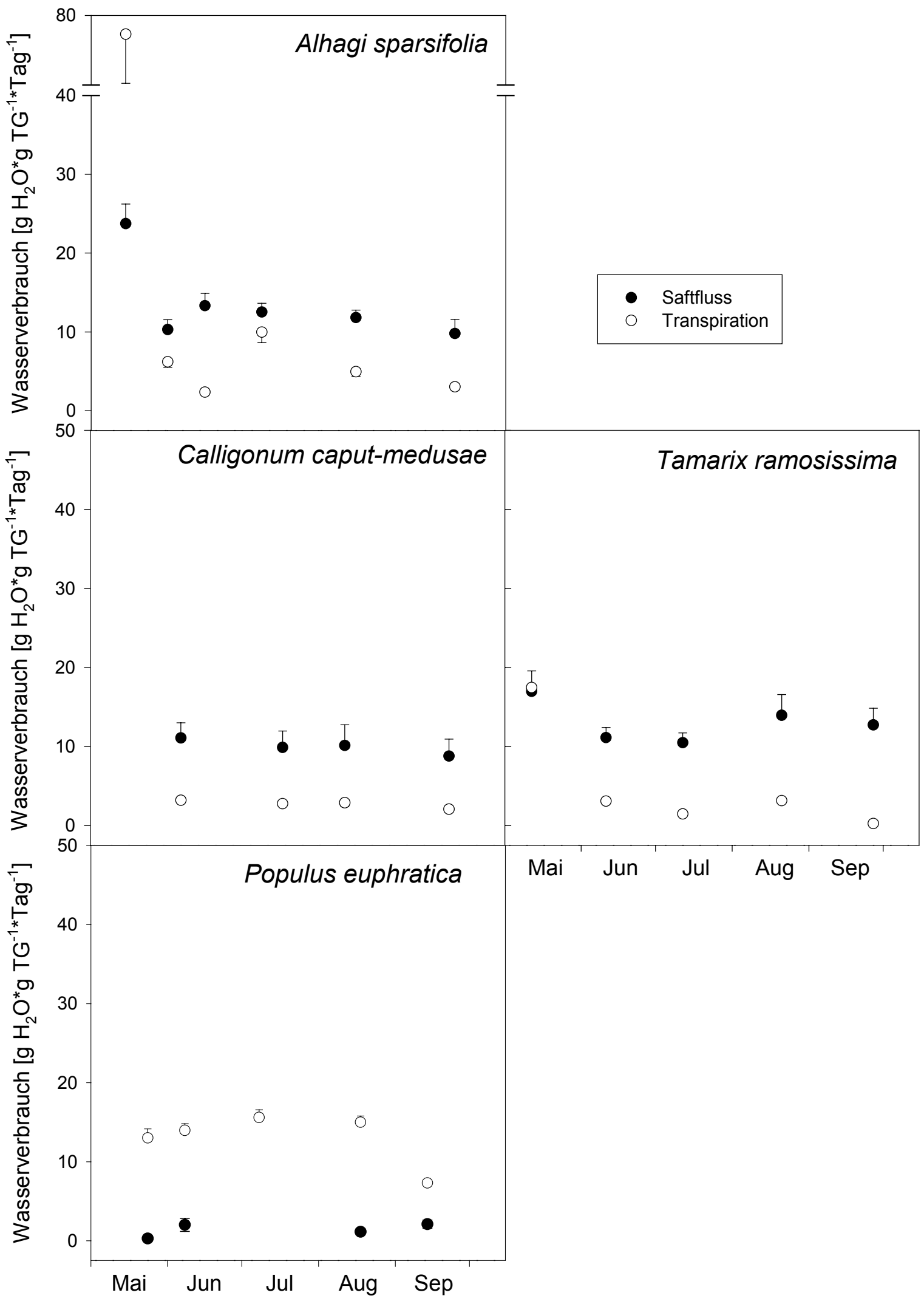

Abb. 4.6.9: Vergleich des Wasserverbrauchs gemessen durch Saftfluss und Transpiration der untersuchten Arten 1999.

Die Transpirationsraten wurden auf die gesamte Tageslänge hochgerechnet. Die Saftflussmenge bezieht sich auf den gleichen Zeitraum. 


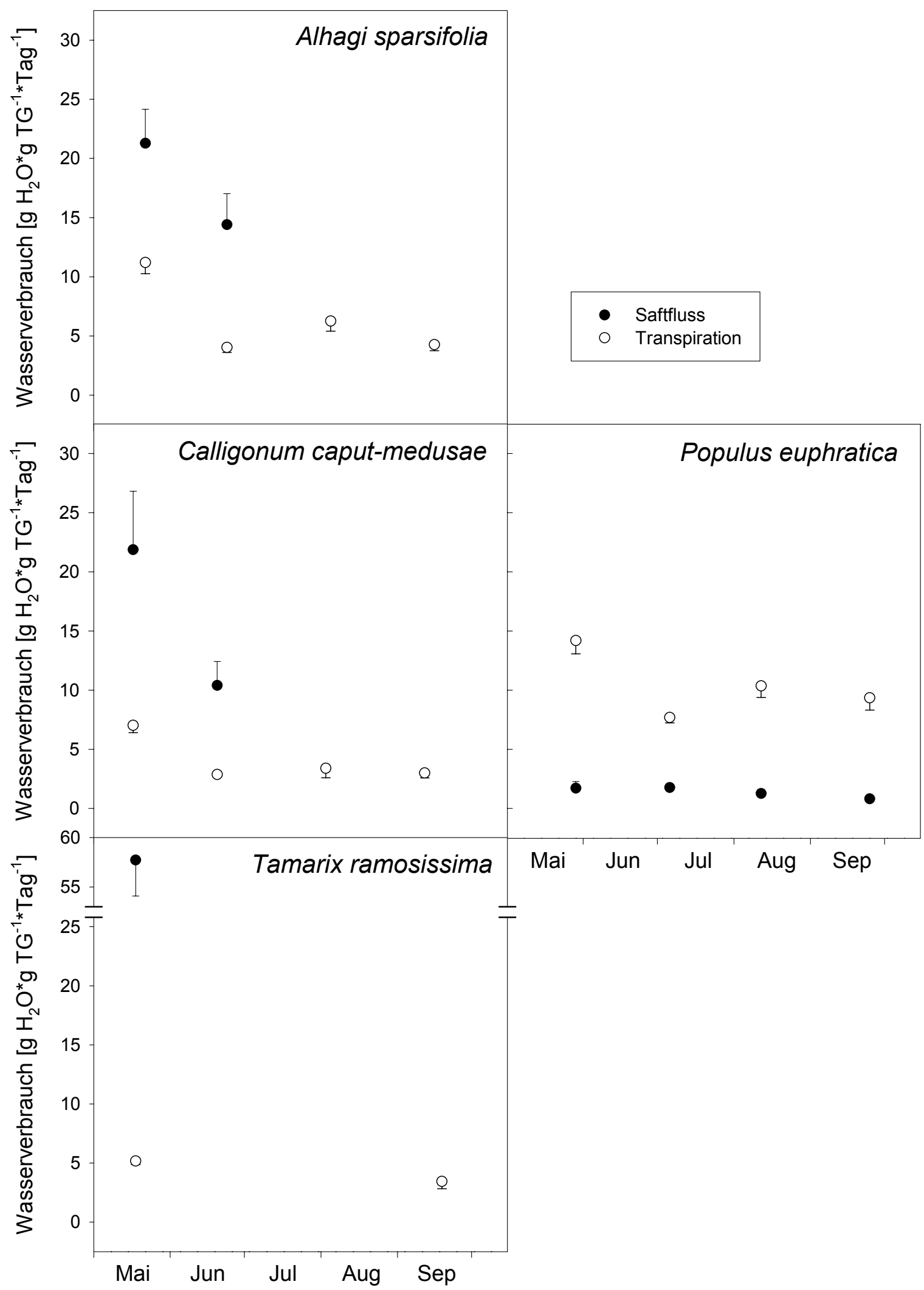

Abb. 4.6.10: Vergleich des Wasserverbrauchs gemessen durch Saftfluss und Transpiration der untersuchten Arten 2000.

Die Transpirationsraten wurden auf die gesamte Tageslänge hochgerechnet. Die Saftflussmenge bezieht sich auf den gleichen Zeitraum. 
Auch für das Jahr 2000 wurde der aus Saftfluss- und Transpirationsmessungen errechnete Wasserverbrauch verglichen (Abb. 4.6.10). Für A. sparsifolia, C. caputmedusae und $T$. ramosissima konnte nur für die Termine in der ersten Hälfte des Jahres eine Saftflussmenge angegeben werden. Wie schon 1999 lagen bei diesen Arten die Saftllusstagessummen auch 2000 über den Transpirationsmengen.

Bei $P$. euphratica blieb wiederum die Saftflussmenge über die Vegetationsperiode gleich und lag unter den Transpirationssummen.

Für einen Methodenvergleich der Transpirationsmessungen mit dem LI-1600 und der Saftflussmessungen mit dem System nach KUČERA wurde der ermittelte Wasserverbrauch von A. sparsifolia, C. caput-medusae und T. ramosissima an allen Messtagen der Jahre 1999 und 2000 gegeneinander aufgetragen. Bezieht man nur die Messungen mit der Koniferenkammer des LI-1600 ein, kann man das Verhältnis der Messungen mit einer Geraden beschreiben (Abb. 4.6.11). Dies weist auf einen systematischen Fehler bei den Transpirations- oder Saftfluss-messungen hin.

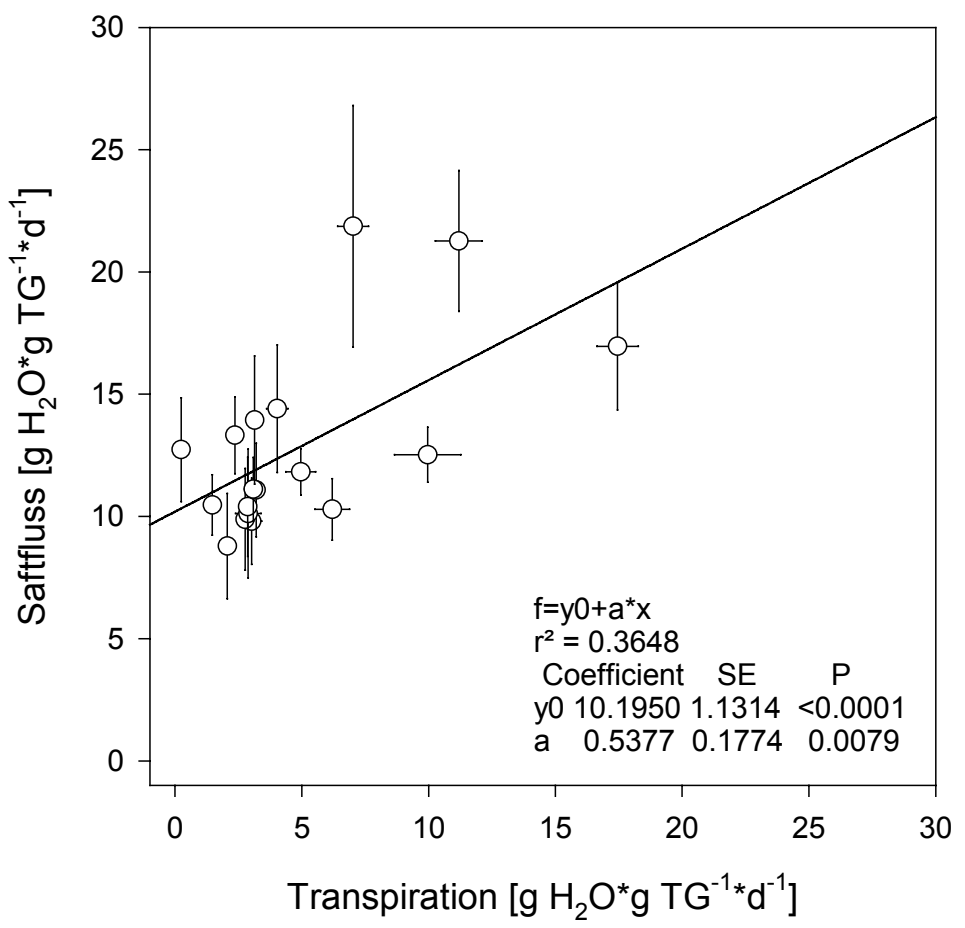

Abb. 4.6.11: Methodenvergleich der Messung des Tageswasserverbrauchs gemessen mit dem Saftflusssystem nach KUČERA und mit dem LI-1600. Es wurden die Messwerte der Arten A. sparsifolia, C. caput-medusae und T. ramosissima aus beiden Untersuchungsjahren verwendet. Die Transpirationsraten wurden auf die gesamte Tageslänge hochgerechnet. 


\subsection{Wassernutzungseffizienz der Produktion (WUE $)$}

Die Wassernutzungseffizienz der untersuchten Pflanzenarten ist in Abb. 4.7.1 dargestellt. Die $\mathrm{C}_{4}$-Pflanze C. caput-medusae weist mit $1,16 \mathrm{~g} \mathrm{TG}^{*} \mathrm{~kg}^{-1} \mathrm{H}_{2} \mathrm{O}$ erwartungsgemäß eine höhere WUEp auf, während $A$. sparsifolia und $T$. ramosissima nur Werte von 0,6 bzw. 0,77 $\mathrm{g} \mathrm{TG}^{*} \mathrm{~kg}^{-1} \mathrm{H}_{2} \mathrm{O}$ erreichen. Für $P$. euphratica konnte 1999 aufgrund der lückenhaften Saftflussmessungen keine Wassernutzungseffizienz berechnet werden. Wenn man zwischen den Messausfällen und zum Anfang und Ende der Vegetationsperiode interpoliert, kann man den Wasserverbrauch sehr grob hochrechnen. Auf diese Weise erhält man für die WUEp einen geschätzten Wert von ca. 3-4 g TG* $\mathrm{kg}^{-1} \mathrm{H}_{2} \mathrm{O}$ (nicht dargestellt).

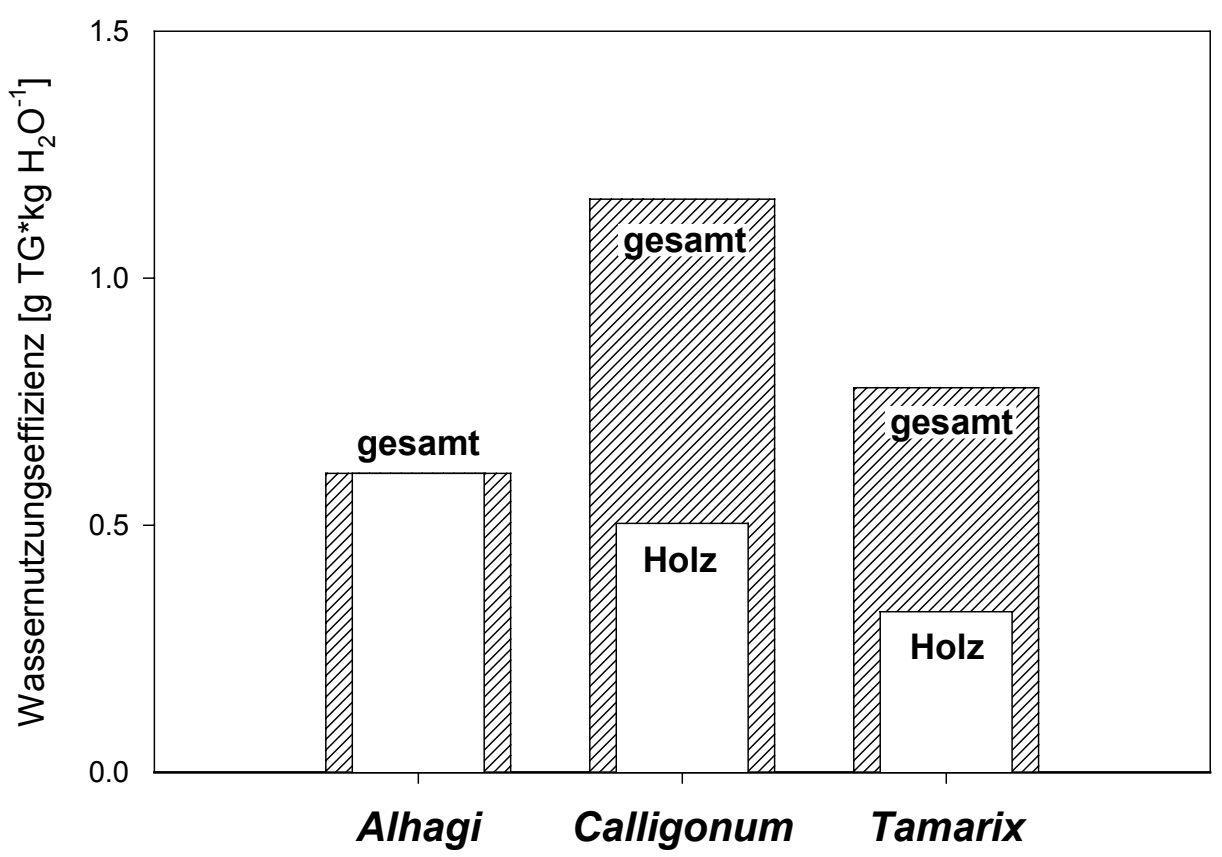

Abb. 4.7.1: Wassernutzungseffizienz der Produktion (WUEp) von A. sparsifolia, $C$. caput-medusae und $T$. ramosissima 1999.

Die Werte sind nur auf die Holzbiomasse bezogen und auf Holz- und transpirierende Biomasse (gesamt) bezogen dargestellt. Die unterirdische Biomasse ging nicht in die Berechnung ein. Für Populus euphratica konnte wegen der fehlerhaften Saftflussmessung keine WUEp berechnet werden.

Im Jahr 2000 konnte nur der Wasserverbrauch von zwei Pappeln berechnet werden. $P$. euphratica zeigte eine mit 3,2 bis $5,5 \mathrm{~g} \mathrm{TG}^{*} \mathrm{~kg}^{-1} \mathrm{H}_{2} \mathrm{O}$ sehr hohe WUEp. Es kann aber davon ausgegangen werden, dass auch in diesem Jahr der Saftfluss 
bei $P$. euphratica unterschätzt wurde, da verglichen mit der Transpiration ein sehr niedriger Wasserverbrauch errechnet wurde (siehe Abb. 4.6.9 und Kapitel 5.4.2). Der Vergleich mit den anderen Arten ist nicht möglich, da für diese im Jahr 2000 keine vollständigen Saftflussdaten vorliegen.

Vergleicht man die WUEp der nutzbaren Teile der Pflanzen, so schneidet $A$. sparsifolia im Vergleich mit den anderen Arten deutlich besser ab, da die gesamte oberirdische Biomasse genutzt wird. Betrachtet man die auf das Holz bezogene WUEp von $C$. caput-medusae und $T$. ramosissima, so zeigt auch hier die $\mathrm{C}_{4-}$ Pflanze einen höheren Wert.

\subsection{Kohlenstoffisotopenverhältnis $\left(\delta^{13} \mathrm{C}\right)$}

Im Verlauf der Vegetationsperiode 1999 wurden die Kohlenstoff-Isotopenverhältnisse bei allen untersuchten Arten zunehmend negativer. Zunächst werden die $\delta^{13} \mathrm{C}$-Werte der $\mathrm{C}_{3}$-Pflanzen vorgestellt, dann die der $\mathrm{C}_{4}$-Pflanze Calligonum caputmedusae.

Alhagi sparsifolia hatte im April 1999 einen $\delta^{13} \mathrm{C}$-Wert von -24,16\% auf der AFund $-24,49 \%$ auf der AD-Fläche (Abb. 4.8.1). Auf beiden Flächen sank der Wert auf etwa $-27 \%$ im Herbst ab. Die beiden Teilflächen unterschieden sich an keinem Termin signifikant. Auf der AF-Fläche war der Termin vor der Überflutung signifikant positiver als der nächstfolgende. Diese Differenz der Isotopenverhältnisse ist aber eher durch den Jahresverlauf der $\delta^{13} \mathrm{C}$-Werte bedingt als durch die Bewässerung. Bei allen untersuchten Arten war der Wert im Mai weniger negativ als im Juni, nur fand bei den anderen Arten die Überflutung später im Jahr statt.

Tamarix ramosissima zeigte im April $1999 \delta^{13} \mathrm{C}$-Werte von -23,48 \%o auf der unbewässerten Fläche und $-24,14 \%$ auf der bewässerten Fläche (Abb. 4.8.1). Im Lauf der Vegetationsperiode fielen die Werte auf -26,40\% auf der TF-Fläche und $-25,43 \%$ auf der TD-Fläche ab. Als einzige der untersuchten Arten unterschieden sich bei $T$. ramosissima an allen Terminen außer im April trockene und überflutete Fläche signifikant: die trockene Fläche hatte durchgehend positivere Isotopenverhältnisse. Diese Differenz zwischen bewässerter Fläche und Kontroll- 
fläche wurde allerdings nicht durch die Bewässerung verursacht, denn sie bestand schon vor der experimentellen Überflutung.
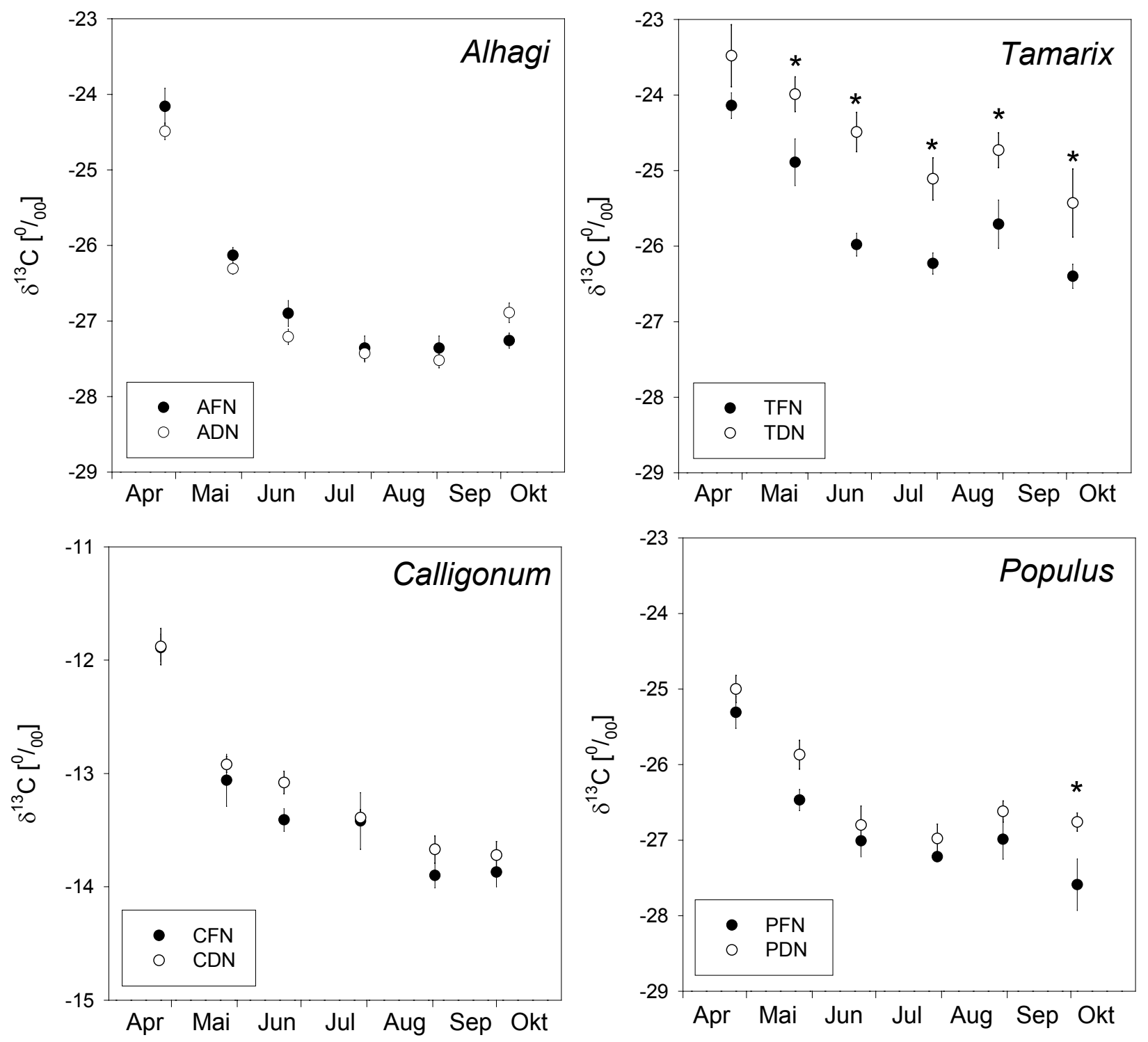

Abb. 4.8.1: Kohlenstoff-Isotopenverhältnisse $\left(\delta^{13} \mathrm{C}\right)$ der vier untersuchten Arten im Jahresverlauf 1999.

Der $\delta^{13} \mathrm{C}$-Wert der Blätter wurde auf den bewässerten ungenutzten (FN) und den unbewässerten ungenutzten (DN) Flächen gemessen. Die Proben auf den Teilflächen wurden am gleichen Tag genommen. Sternchen (*) kennzeichnen einen signifikanten Unterschied zwischen den FN- und DNFlächen am gleichen Messtermin. 
Populus euphratica wies mit - 25,31\% (PF-Fläche) bzw. -25,00 \%00 (PD-Fläche) die negativsten Frühjahrswerte auf. Die nicht bewässerte Fläche hatte tendenziell etwas positivere Isotopensignaturen. Nur am letzten Termin (04.10.1999) wurden auf der unbewässerten Fläche signifikant positivere $\delta^{13} \mathrm{C}$-Werte gemessen.

Calligonum caput-medusae zeigte als $\mathrm{C}_{4}$-Pflanze deutlich positivere Werte als die drei untersuchten $\mathrm{C}_{3}$-Pflanzen. Von - 11,89 \%0 auf der bewässerten Fläche bzw. $11,88 \%$ auf der unbewässerten Fläche im April sank das Isotopenverhältnis auf $13,9 \%$ (CF-Fläche) und - 13,72\% (CD-Fläche) ab. An keinem der Termine im Verlauf der Vegetationsperiode unterscheiden sich die Werte der trockenen und überfluteten Fläche signifikant.

Von A. sparsifolia wurden auch 2000 Proben genommen. Der Wert im April war auf der AFN-Fläche deutlich negativer als im Vorjahr, auf den anderen Teilflächen wurden sehr ähnliche Werte gemessen (Abb. 4.8.2). Im Spätsommer bzw. Herbst wurden ebenfalls etwas negativere Werte als 1999 ermittelt.

Auf der ADN-Fläche fällt der mit - 26,4 \% wenig negative Wert im August auf, der vermutlich auf eine Probenahme jüngerer Blätter zurückzuführen ist. Signifikante Unterschiede zwischen den bewässerten und unbewässerten Flächen treten sonst nicht auf.

Zwischen den genutzten und ungenutzten Teilflächen (AFN und AFU) bestand nur im August ein signifikanter Unterschied, hier war der Wert auf der AFU-Fläche deutlich positiver. In den anderen Monaten waren sich die Isotopensignaturen sehr ähnlich. Bei den unbewässerten Flächen wies die genutzte Teilfläche (ADU) generell etwas negativere Werte auf als die ungenutzte Teilfläche ADN (signifikant aber nur im August). 


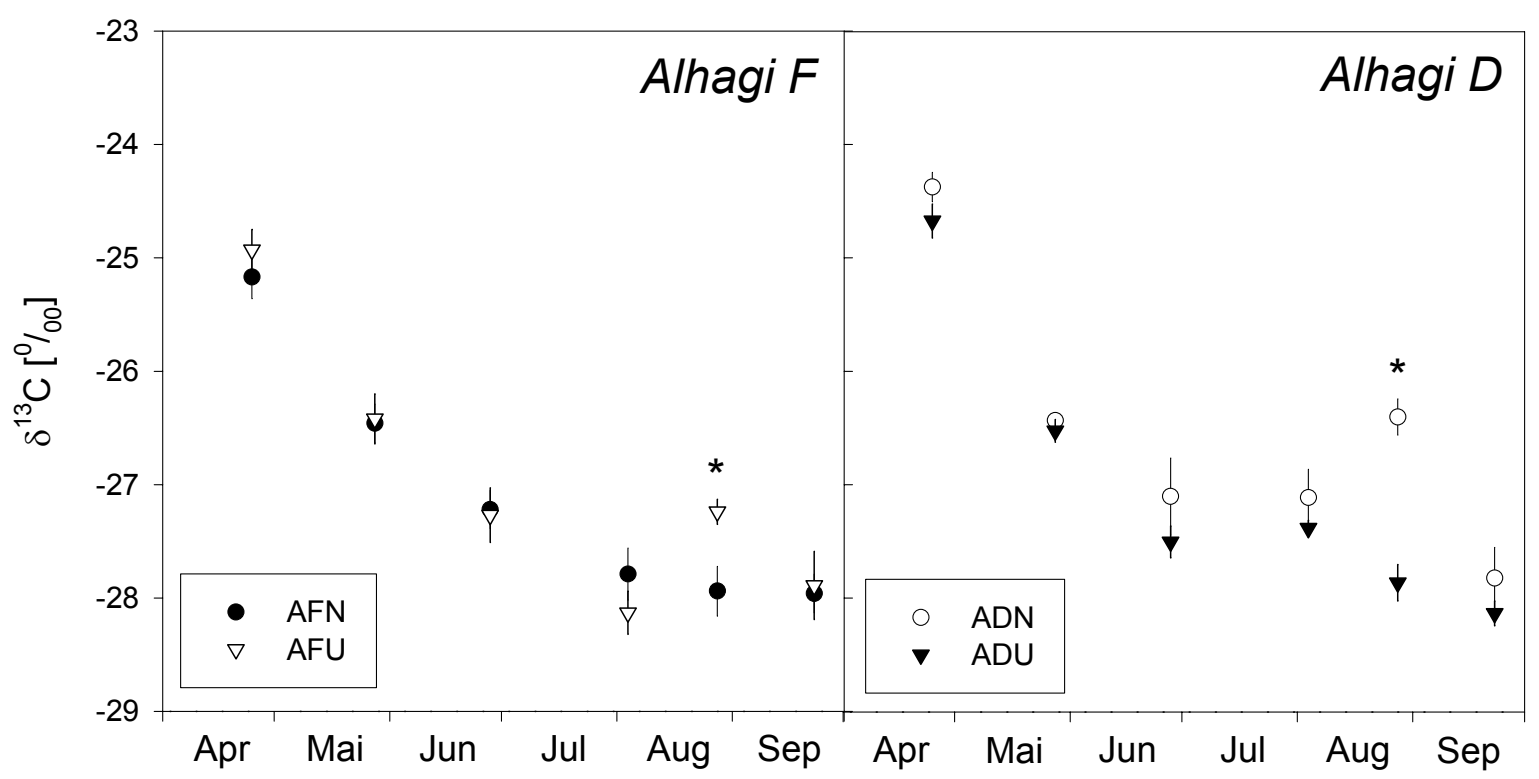

Abb. 4.8.2: Kohlenstoff-Isotopenverhältnisse $\left(\delta^{13} C\right)$ von $A$. sparsifolia im Jahr 2000.

Der $\delta^{13} \mathrm{C}$-Wert der Blätter wurde auf den bewässerten ungenutzten (AFN) und genutzten (AFU) sowie auf den unbewässerten ungenutzten (ADN) und genutzten (ADU) Flächen gemessen. Die Proben auf den Teilflächen wurden am gleichen Tag genommen. Sternchen $\left(^{*}\right)$ kennzeichnen einen signifikanten Unterschied zwischen den in der gleichen Grafik dargestellten Flächen am gleichen Messtermin. 


\section{Diskussion}

\subsection{Die Oase Qira als Modell für die Desertifikations- Problematik am Südrand der Taklamakan}

Am Südrand der Taklamakan gibt es einen Niederschlagsgradienten von Westen nach Osten (YANG 1991): Während in Kashgar (chin. Kashi) 65,4 mm Niederschlag gemessen wurden, sind es in Qiemo nur 19 mm (Karte: Abb. 2.1.1). Im Inneren der Taklamakan fallen noch weniger Niederschläge. Der Nordrand der Wüste erhält etwas höhere Niederschläge als der Südrand und wird vom Tarim mit mehr Wasser versorgt. Die Zuflüsse des Tarim, die aus dem Tian Shan kommen, haben aber eine geringere Wasserführung als die Flüsse, die im Kunlun entspringen (WALTER \& BOX 1983).

Die jährliche potentielle Evaporation am Südrand der Wüste erreicht Werte von 1640 bis 2830 mm (Xia et al. 1993). Die landwirtschaftliche Struktur der Oasen am Südrand der Taklamakan ist untereinander vergleichbar. Neben dem Bewässerungsfeldbau spielt die Viehhaltung und Weidewirtschaft eine wichtige Rolle. Das Oasenvorland hat als Schutz vor Sanddrift und Quelle für Brenn- und Bauholz sowie Viehfutter in allen Oasen eine große Bedeutung.

Der Qira-Fluss hat im Vergleich zu dem benachbarten Hotan-Fluss (im Westen) oder dem Keriya (im Osten) ein kleines Wassereinzugsgebiet, die jährlich abfließende Wassermenge ist somit geringer (COQUE et al. 1991). Dementsprechend ist die Bevölkerungszahl und die bewässerte Ackerfläche der Oase Qira kleiner als die der benachbarten Oasen. Es ist möglich, dass das Vorland von Qira verglichen mit den umliegenden Oasen von weniger Oberflächenwasser erreicht wird.

Da am gesamten Südrand der Taklamakan ähnliche Bedingungen hinsichtlich des Klimas und der Wasserverfügbarkeit herrschen, treten in den dortigen Oasen auch vergleichbare Probleme bei der Wasserversorgung und Nutzung der Vorlandvegetation auf. Die Oase Qira kann also als Modell für den gesamten Südrand der Taklamakan gelten. Die Vegetation in der Region ist zwar artenärmer, aber im übrigen anderen zentralasiatischen Wüsten sehr ähnlich (WALTER \& BOX 1983). Deswegen kann man davon ausgehen, dass die Ergebnisse sich auch auf andere Gebiete übertragen lassen. 


\subsection{Wasserverfügbarkeit für Pflanzen im Oasenvorland von Qira}

\section{Bedingungen ohne Bewässerung}

Die jährliche Niederschlagssumme im Gebiet ist mit nur $35 \mathrm{~mm}$ sehr gering und bei einer potentiellen Evaporation von etwa $2600 \mathrm{~mm}$ (XIA et al. 1993) für die pflanzliche Wasserversorgung praktisch unbedeutend. Daher wurde zu Versuchsbeginn erwartet, dass die Wasserversorgung der Pflanzen im Vorland ausschließlich durch einen von gelegentlichen Überflutungen aufgefüllten Wasservorrat im Boden erfolgt, der mit zunehmendem zeitlichen Abstand der letzen Überflutung abnimmt, so dass die Pflanzen unter zunehmendem Trockenstress leiden, ihr Wasserverbrauch abnimmt und sie Mechanismen der Trockenresistenz entwickeln.

Auf allen untersuchten Flächen war allerdings die in den oberen Bodenschichten vorhandene Wassermenge vor der Bewässerung 1999 und im Jahr 2000 sehr gering. Es wurden mit den TDR-Sonden nur Werte unter 5 Vol. \% gemessen, abgesehen vom Einflussbereich des Grundwassers, das bei Bohrungen auf der Populus- und Tamarix-Fläche erreicht wurde (Abb. 4.2.1). Auch gravimetrische Bodenwassergehaltsmessungen ergaben bis in eine Tiefe von 7,7 m nur Werte von 0 bis 1,8 \% auf einer Untersuchungsfläche in Oasennähe (ARNDT, S.K. unveröffentlicht).

Die Wassergehalte zeigten weder im Bereich des Grundwassersaumes noch in den darüber liegenden Bodenschichten eine Veränderung während der Vegetationsperiode 2000, die bei Absinken des Grundwassers oder beim Verbrauch eines vorhandenen Bodenwasservorrats durch die Pflanzen auftreten würde, sondern blieben auf einem Niveau.

Die Eindringtiefe der seltenen Niederschlagsereignisse war sehr gering und erreichte höchstens $15 \mathrm{~cm}$ (eigene Beobachtungen). Oberflächenwasser kann nur sehr langsam in den Boden eindringen, da keine Struktur mit Grob- und Mittelporen vorliegt.

Auch Taufall, wie er von vielen Wüsten und Halbwüsten bekannt ist (LÖSCH 2001), kann aufgrund der Klimamessungen und eigener Beobachtungen ausgeschlossen 
werden. Die relative Luftfeuchte stieg selbst im Morgengrauen, wenn die geringsten Temperaturen auftraten, meist nicht über 60 oder $70 \%$ an. Wasserdampfsättigung der Luft trat außer bei kurzzeitigen Regenfällen nie auf.

Die Pflanzen in Wüsten mit regelmäßig auftretenden Niederschlägen, wie z.B. der Sonaran Desert (USA) mit 50-300 mm Niederschlag oder dem Colorado Plateau (USA), sind an eine Wasserversorgung aus unterschiedlichen Quellen angepasst. Sie haben zusätzlich zu einem tiefstreichenden Wurzelsystem oberflächennahe Wurzeln, die die Niederschlagspulse nutzen können (z.B. SALA et al. 1996, Gebauer \& EhleRINGer 2000, SchWINNING \& EhLERINGer 2001). Bei dem hier vorgestellten Projekt wurden weder bei den Bohrungen zum Einsetzen der TDRSonden noch bei anderen Bodenuntersuchungen lebende oder tote Feinwurzeln in den oberen Bodenschichten gefunden. Beim Graben von mehreren bis zu 3,50 m tiefen Gruben wurden lediglich Rhizome (bei $A$. sparsifolia) oder von Bodenmaterial überlagerte unter-irdische Triebe sowie Wurzelschösslinge gefunden. Nur auf der Tamarix-Fläche wurden bei Bohrungen zum Einbringen der TDR-Sonden bis in etwa drei Meter Tiefe vereinzelt abgestorbene Wurzeln entdeckt, die noch oberhalb des Kapillarsaums des Grundwassers lagen. Das Wachstum dieser Wurzeln könnte durch eine frühere Überflutung oder einen Starkregen induziert worden sein.

Eine weitere Möglichkeit der Wassererschließung ist das häufig in Trockengebieten beobachtete Phänomen des „hydraulic lift“. Hierbei wird Wasser von Wurzeln in tiefer gelegenen Bodenschichten mit einem höheren Wassergehalt aufgenommen und entsprechend dem Gefälle des Wasserpotentials im Wurzelsystem zu Feinwurzeln im trockenen Oberboden transportiert. So können diese Wurzeln mit Wasser versorgt werden. Darüber hinaus kann Wasser in den trockenen Boden entlassen werden und (diurnale Zyklen des Wasserpotentials vorausgesetzt) später wieder, möglicherweise angereichert mit Nährstoffen, aufgenommen werden (CALDWELL, DAWSON \& RICHARDS 1998, BURGESS et al. 1998, YODER \& NOWAK 1999b, BURGESS et al. 2000, BURGESS et al. 2001).

Bei einer Untersuchung am Oasenrand von Qira an Eleagnus angustifolia, Calligonum caput-medusae, Alhagi sparsifolia und Karelinia caspica im Sommer 2000 wurden bei Grabungen wie schon auf den Flächen im Vorland keine oberflächen- 
nahen lateralen Wurzeln gefunden, an denen man mit Saftflussmessungen hydraulic lift hätte nachweisen können (LANDMAN, P. unveröffentlicht). Es wurden ebenfalls bis in $50 \mathrm{~cm}$ Tiefe keine Feinwurzeln gesehen.

Es kann also kein hydraulic lift bei den untersuchten Pflanzen angenommen werden, da weder Wurzeln noch ein höherer Bodenwassergehalt in den Bodenschichten oberhalb des Grundwassersaumes gefunden wurden.

Die Pflanzen im Oasenvorland waren vital und zeigten keine Anzeichen für starken Trockenstress. Es muss also davon ausgegangen werden, dass die Pflanzen das Grundwasser erreichen und dort ihren Wasserbedarf decken. Bei zwei der untersuchten Arten wurde das Grundwasser bei Bohrungen auf den Untersuchungsflächen erreicht:

Auf der bewässerten Tamarix-Fläche (TF) stand das Grundwasser unbeeinflusst von der Bewässerung in 6,4 $\mathrm{m}$ an, dort hatte $T$. ramosissima auf jeden Fall Zugang zu wassergesättigten Bodenschichten. Aber auch bei weiteren Untersuchungen auf hohen von $T$. ramosissima bewachsenen Dünen mit einem Abstand von 23,2 $\mathrm{m}$ zum Grundwasser muss nach vorliegenden Ergebnissen davon ausgegangen werden, dass T. ramosissima das Grundwasser erreicht (GRIES et al. 2003). Auf der bewässerten Populus-Fläche (PF) wurde der Grundwassersaum vor der Bewässerung in 3,6 m Tiefe gefunden, aber selbst auf Dünen mit größerer Entfernung zum Grundwasser (bis zu $23 \mathrm{~m}$ ) wurden vitale Pappeln vorgefunden, die nicht unter starkem Trockenstress litten (GRIES et al. 2003).

Bei $A$. sparsifolia dagegen konnte bei Bohrungen kein Hinweis dafür gefunden werden, dass die Pflanzen Zugang zum Grundwasser haben. Bei der AlhagiFläche muss, wenn man den Wasserstand in einem nahe gelegenen Brunnen heranzieht, davon ausgegangen werden, dass das Grundwasser in ca. $16 \mathrm{~m}$ Tiefe ansteht. Von Alhagi pseudalhagi (wahrscheinlich mit $A$. sparsifolia identisch) ist schon lange bekannt, dass die Wurzeln tiefe wasserführende Schichten erschließen (KeLLER 1925) bzW. bis zu 15 m Tiefe erreichen (SHMUel 1948). Man kann also annehmen, dass $A$. sparsifolia auch auf den Untersuchungsflächen das Grundwasser erreicht.

Bei den Bohrungen auf der Calligonum-Fläche wurde ebenfalls nicht der Kapillarsaum des Grundwassers erreicht. Das Grundwasser muss unter einer Tiefe von 8 $\mathrm{m}$ anstehen, da auf der benachbarten vegetationsfreien Fläche (open ground) bei 
einer Bohrung bis in fast $8 \mathrm{~m}$ Tiefe der Grundwassersaum nicht erreicht wurde. Ausgehend von der Tiefe nahegelegener Brunnen, wird das Grundwasser in etwa $12 \mathrm{~m}$ Tiefe vermutet.

\section{Bedingungen mit Bewässerung}

Die natürlich auftretenden Sommerhochwasser der aus den umliegenden Bergen kommenden Flüsse sind sehr variabel, wie aus der Oase Qira (XIA et el. 1993) und von Untersuchungen am etwas weiter östlich fließenden Keriya bekannt (CHENG 1991a und 1991b, TIAN 1991). Im Gegensatz zu den ursprünglichen Erwartungen erreichten die Überflutungen nicht großflächig das Oasenvorland, sondern betrafen weitgehend nur die Flussbetten und angrenzende Niederungen. Im Qira-Fluss fließt in manchen Jahren (wie z.B. 1999) im Sommer nur so viel Wasser, dass es komplett in der Oase verbraucht wird und nichts durch das Flussbett oder die Bewässerungskanäle ins Vorland abfließt. Bedingt durch das Relief des Oasenvorlandes hat der Großteil der natürlichen Vegetation aber auch dann keinen Zugang zu diesem Oberflächenwasser, wenn in einem Jahr mit reichlicher Wasserführung (z.B. 2000) der Qira-Fluss bis weit in die Wüste hinaus fließt und überschüssiges Wasser aus der Oase durch die in Wadis mündenden Abflusskanäle ins Vorland gelangt. Es kann also ausgeschlossen werden, dass das Überleben der Vorlandvegetation von der Zufuhr von Oberflächenwasser bei Überflutungen abhängig ist.

Das im Methodenteil beschriebene Bewässerungsexperiment wurde angelegt, um die Reaktion der Pflanzen auf ein größeres Wasserangebot und die anschließende Austrocknungsphase zu untersuchen.

Das Überflutungswasser drang maximal bis in eine Tiefe von $2 \mathrm{~m}$ vor (Abb. 4.2.1). Aus pF-Kurven von Bodenproben aller Untersuchungsflächen kann man ersehen, dass selbst die Überflutung kaum pflanzenverfügbares Wasser bereitgestellt hat (THOMAS, F.M. unveröffentlicht). Es ist also schon allein von den Bodenwasserpotentialen her unwahrscheinlich, dass die untersuchten Pflanzenarten von einer Bewässerung wie in diesem Projekt profitieren können. 
Die Infiltrationsrate des Wassers ist gering, da der Boden sehr homogen aus $87 \%$ Grobschluff besteht und keine Struktur mit Grob- und Mittelporen vorliegt. Anders als in Sandböden kann das Wasser nicht rasch in größere Tiefen geleitet und dort gespeichert oder zum Dünenfuß hin abgeleitet werden, wie es zum Beispiel in sandigen Dünensystemen der Negev (VESTE \& BRECKLE 1995, 1996) beobachtet wurde. An den TDR-Messungen auf der Tamarix-Fläche 1999 ist zu sehen, dass es offenbar keinen lateralen Wassertransport im Boden gab, denn das Überflutungswasser hatte keinen Einfluss auf den Bodenwassergehalt unter der nahe gelegenen Düne, obwohl es in einer Entfernung von nur einem Meter von den TDR-Sonden anstand.

Einen Beitrag zum schnelleren Eindringen von Wasser in tiefere Bodenschichten können Makroporen leisten, die an Stelle von abgestorbenen Wurzeln oder unterirdischen Sprossen entstehen, oder auch Gänge von Bodenwühlern (DEVITT \& SMITH 2002). Auf den Untersuchungsflächen kann dies vor allem auf der AlhagiFläche eine Rolle gespielt haben, da A. sparsifolia ein unterirdisches Netzwerk von Sprossen ausbildet. Außerdem wurden auf dieser Fläche häufig Nager beobachtet und viele Grabgänge gefunden. Auch auf der Freifläche könnte das rasche Eindringen des Wassers bis in $1 \mathrm{~m}$ Tiefe mit vorhandenen Makroporen erklärt werden. Obwohl die Fläche 1999 vegetationsfrei war, wuchsen im Folgejahr (2000) durch den Beweidungsausschluss begünstigt, zahlreiche Individuen von Launaea polydichotoma aus unterirdischen Trieben auf, die also schon früher vorhanden gewesen sein mussten. Die nach der Zersetzung abgestorbener unterirdischer Triebe verbliebenen bzw. entstandenen Hohlräume könnten den schnelleren vertikalen Wassertransport ermöglicht haben.

\section{Konsequenzen für die Fragestellung}

Schon aus den Geländeeigenschaften des Oasenvorlandes ist zu folgern, dass Überflutungen für die Existenz bereits etablierter ausdauernder Pflanzenbestände nicht notwendig ist. Die Untersuchung der Bodenwassergehalte und Bodenwasserpotentiale bestätigt diese Folgerung. Die räumliche Verteilung der Pflanzen folgt nicht den Niederungen und Flussverläufen, sondern muss von anderen Faktoren bestimmt werden. Da man ausschließen kann, dass die Vegetation von 
Niederschlägen abhängt, bleibt nur die Möglichkeit, dass die untersuchten Arten das Grundwasser erreichen. Es ist davon auszugehen, dass die Grundwassertiefe an einigen Standorten für das Pflanzenwachstum limitierend ist.

Weitere Belege dafür, dass die Pflanzen vom Grundwasser abhängig sind, können Untersuchungen des Wasserhaushalts der Pflanzenarten liefern. Diese Untersuchungen können gleichzeitig Hinweise zur saisonalen Verfügbarkeit des Grundwassers geben. Es ist nicht bekannt, ob sich die durch Wasserentnahme in der Oase entstehende Schwankung des Grundwasserspiegels um mehrere Meter bis ins Oasenvorland fortsetzt. Die Wasserhaushaltsuntersuchungen können Auskunft über den Wasserverbrauch der Arten bei der hohen Transpirationsbelastung im Untersuchungsgebiet und über möglicherweise unterschiedliche Strategien der untersuchten Arten bei der Regelung ihrer Wasserbilanz geben.

Außerdem besteht die Möglichkeit, dass eine Bewässerung zu Keimungserfolgen führt und so zur Regeneration der Vorlandvegetation beitragen kann.

\subsection{Wasserhaushalt der Pflanzen}

\subsubsection{Höhe und zeitlicher Verlauf der Wasserpotentiale}

Es wird angenommen, dass sich das predawn-Blattwasserpotential $\left(\Psi_{\mathrm{pd}}\right)$ bei ausreichender Wasserversorgung im Gleichgewicht mit dem Wasserpotential des direkt die Wurzeln umgebenden Bodens befindet (DAVID et al. 1997, AMÉGLIO et al. 1999). Daher sinkt $\Psi_{p d}$ bei einer Verringerung des Bodenwassergehaltes ab und eine Aufsättigung des Gewebes über Nacht ist nicht mehr möglich (BRÉDA et al. 1993, ARNDT et al. 2001, COMSTOCK 2002, SPERRY et al. 2002). Schon bei höheren Bodenwasser-gehalten kann es durch nächtliche Transpiration oder eine nicht ausreichende Aufsättigung durch zu langsame Wassernachlieferung zu einem Ungleichgewicht zwischen Boden- und predawn-Blattwasserpotential kommen (DonOvan et al. 1999).

Aufgrund der Annahme, dass die Pflanzen Grundwasseranschluss haben, wurde erwartet, dass die untersuchten Pflanzenarten nicht so niedrige predawn-Wasserpotentiale entwickeln würden wie Pflanzen in Trockengebieten mit unregelmäßiger 
Wasserversorgung. Durch die längeren Leitungswege wurden jedoch bei zunehmendem Abstand zum Grundwasser niedrigere Wasserpotentiale erwartet.

Die predawn-Wasserpotentiale in der vorliegenden Arbeit nahmen im Laufe der Vegetationsperiode ab (Abb. 4.4.5). Bei drei der untersuchten Arten sanken die $\Psi_{\text {pd }}$ aber nicht unter -1 MPa. Sogar Tamarix ramosissima, die hohe Salzgehalte aufwies und bei der man deshalb bei vergleichbarer Bodenwasserverfügbarkeit noch niedrigere Wasserpotentiale erwarten würde, wies nur $\Psi_{\mathrm{pd}}$ bis -1,22 MPa auf. Selbst mitteleuropäische Bäume können niedrigere Werte erreichen z.B. Quercus petraea mit -2,0 MPa nach einer Periode von Trockenstress (BRÉDA et al. 1993). Offensichtlich haben die im Oasenvorland von Qira untersuchten Arten während der ganzen Vegetationsperiode keine eingeschränkte Wasserverfügbarkeit im Wurzelraum.

Von Arten in Trockengebieten wird berichtet, dass sie im Tagesverlauf zum Teil sehr niedrige Wasserpotentiale erreichen: LARCHER (1994) listet für Pflanzen periodisch trockener Gebiete $-3,5$ bis $-8,5 \mathrm{MPa}$, für Wüstensträucher (Xerophyten) -5 bis $-8 \mathrm{MPa}$ (in Extremfällen bis $-16 \mathrm{MPa}$ ) und für Halophyten -3 bis $-6 \mathrm{MPa}$ (Extremwert -9 MPa) auf. Dagegen wurden in der vorliegenden Untersuchung die niedrigsten Mittagswasserpotentiale im Artvergleich bei Tamarix ramosissima mit nur etwa -3 MPa gemessen. Bei einem VPD $\mathrm{max}_{\text {von }} 6$ bis $7 \mathrm{kPa}$ an Sommernachmittagen erscheinen diese Mittagswasserpotentiale wenig negativ.

Vergleichbare predawn- und Mittagsblattwasserpotentiale wurden z.B. von THOMAS \& EAMUS (2002) im Monsun beeinflussten Nordaustralien oder von BACKES (1996) und CONERS (2001) in der Lüneburger Heide an Rotbuchen (Fagus sylvatica) gemessen.

\section{Tamarix ramosissima}

Der Wasserhaushalt von T. ramosissima wurde seit den 1980er Jahren ausführlich untersucht (z.B. ANDERSON 1982, BROTHERSON \& VON WINKEL 1986, SALA, SMitH \& DeVitt 1996, CleVerly et al. 1997, DeVitT et al. 1997, DeVITt, Smith \& NeumanN 1997, GLADWIN \& RoELLE 1998, GLENN et al. 1998a).

Für Tamarix ramosissima wurden im Südwesten der USA predawnWasserpotentiale von $-0,8$ bis $-0,9 \mathrm{MPa}$ und Mittagswasserpotentiale von $-2,4$ bis 
-2,9 MPa ermittelt (ANDERSON 1982). Dies entspricht den Werten, die im Oasenvorland von Qira gemessen wurden (Abb. 4.4.3). Allerdings beobachtete ANDERSON (1982) eine Mittagsdepression des $\mathrm{CO}_{2}$-Gaswechsels durch absinkende stomatäre Leitfähigkeit, obwohl die Pflanzen ausreichend wasserversorgt waren und die Transpiration auf gleichem Niveau verblieb. HORTON, KOLB \& HART (2001) allerdings berichten von mittleren predawnWasserpotentialen von Tamarix chinensis in Arizona, USA, von -1.64 bis $-2.72 \mathrm{MPa}$ (minimaler Wert etwa $-4 \mathrm{MPa}$ ), letzteres in einem trockenen Jahr bei einer Entfernung von fast $8 \mathrm{~m}$ zum Grundwasser. Aber auch in einer Entfernung von 2-3 m zum Grundwasser wurden ebenso niedrige Wasserpotentiale gemessen. Die Tamarisken waren offensichtlich hauptsächlich an eine Nutzung von Oberflächenwasser angepasst, das in dem beschriebenen Trockenjahr in geringerem Maße vorhanden war. Daher zeigten die Tamarisken deutliche Anzeichen von Trockenstress, wie sehr niedrige Wasserpotentiale und Absterben der Kronen. Im Oasenvorland von Qira zeigten die Tamarisken keine solchen Anzeichen von Trockenstress. Sie waren vital und konnten sogar nach zweimaligem Kahlfraß im Untersuchungsjahr 2000 wieder austreiben (Abb. 4.6.6).

\section{Populus euphratica}

Die gemessenen predawn-Wasserpotentiale von Populus euphratica waren vergleichbar mit denen des in den Trockengebieten der südlichen USA an Flussläufen verbreiteten fakultativen Phreatophyten Populus fremontii (HORTON, KOLB \& HART 2001). P. fremontii zeigt allerdings schon bei Wasserpotentialen von $-1,6 \mathrm{MPa}$ Kavitationen (PoCKMAN et al. 1995), wogegen bei $P$. euphratica z.B. im August 1999, an einem Tag, an dem keinerlei Einschränkungen der stomatären Leitfähigkeit auftraten, sogar Mittagswasserpotentiale von -2,35 MPa gemessen wurden (Abb. 4.4.4). Auf nahegelegenen Dünen mit einem Abstand von etwa $22 \mathrm{~m}$ zum Grundwasser wurden bei $P$. euphratica sogar $\Psi_{m d}$ von -3,05 MPa gemessen (GRIES et al. 2003). Populus euphratica wird von WICKENS (1998) als obligater Phreatophyt bezeichnet. $P$. euphratica scheint also einen höheren Schwellenwert, ab dem Kavitationen auftreten, als $P$. fremontii zu haben, und kann somit auch eine größere Entfernung zum Grundwasser tolerieren. 


\section{Alhagi sparsifolia}

Die Wasserpotentiale in der vorliegenden Untersuchung waren wenig negativ, wenn man die große Entfernung zum Grundwasser und den gleichzeitig hohen Wasserverbrauch von $A$. sparsifolia berücksichtigt (Abb. 4.4.1). Von NILSEN et al. (1983) wurden Wasserpotentiale für den Phreatophyten Prosopis glandulosa gemessen. Diese Fabacee weist in den Sommermonaten bei einer Grundwassertiefe von 4 bis $6 \mathrm{~m}$ ein predawn-Wasserpotential von $-2,5 \mathrm{MPa}$ und mittags $-4,5 \mathrm{MPa}$ als Minimalwert auf. $A$. sparsifolia dagegen erreichte weder so negative $\Psi_{\mathrm{pd}}$ noch $\Psi_{\mathrm{md}}$, im September 1999 wurde das niedrigste Mittagswasserpotential mit $-2,36 \mathrm{MPa}$ bzw. $-2,42 \mathrm{MPa}$ im September 2000 erreicht.

\section{Calligonum caput-medusae}

Bei Calligonum caput-medusae wurden die höchsten Wasserpotentiale gemessen, sowohl im Morgengrauen als auch mittags (Abb. 4.4.3 u. 4.4.6). Die Schwankungen im Jahresverlauf und die Differenzen zwischen $\Psi_{\mathrm{pd}}$ und $\Psi_{\mathrm{md}}$ waren geringer als bei den anderen untersuchten Arten (Tab. 4). C. caput-medusae hat durch den $\mathrm{C}_{4}$-Metabolismus sowie stark reduzierte Blätter und in die photosynthetisch aktiven Sprosse eingesenkte Stomata eine effizientere Anpassung an Wassermangel (siehe auch Kapitel 5.3.3) und eine geringere stomatäre Leitfähigkeit. Daher wird auch ein weniger negatives Wasserpotential aufgebaut. WANG et al. (1997) berichten von einem Absinken der Wasserpotentiale bei Calligonum arborescens nach einer Trockenperiode von 12 Tagen auf $-1,86 \mathrm{MPa}$, einem ähnlichen Wert wie auch in der vorliegenden Arbeit gemessen.

\subsubsection{Anreicherung von Osmotika}

Auch die Anreicherung von osmotisch wirksamen Substanzen in den transpirierenden Organen kann das Wasserpotential beeinflussen. Eine Anreicherung von Osmotika im Cytoplasma und in der Vakuole führt im Allgemeinen zu einem Absinken des osmotischen Potentials und des Wasserpotentials.

Durch Osmoregulation kann der Turgor auch bei trockeneren Bedingungen aufrechterhalten werden. Wichtigstes anorganisches Osmotikum ist Kalium (HSIAO 
\& LÄUCHLI 1986). Auch Chlorid, Nitrat und bei Halophyten Natrium treten als Osmotika auf. Organische Osmotika sind verschiedene Zucker (z.B. Trehalose) und Zuckeralkohole (z.B. Mannit, Pinit), Aminosäuren (z.B. Prolin, Alanin, Taurin), organische Säuren und quartäre Ammoniumverbindungen wie Glycinbetain, Prolinbetain und andere (LösCH 2001).

Vielfach erfolgt aber auch eine passive Anreicherung von Osmotika im Laufe der Alterung von Organen. Der Turgornullpunkt verschiebt sich während dieses Seneszenzverlaufs zu niedrigeren Wasserpotentialen. Vor allem bei Halophyten kann mit zunehmendem Alter Natrium angereichert werden (LÖsCH 2001).

Die untersuchten Arten gelten als Halophyten (A. sparsifolia (KASSAS 1952, KURBAN et al. 1998, JIN 2002, ZHANG, GAO \& ZHENG 2002), T. ramosissima) oder zumindest als salztolerant ( $P$. euphratica). Bei $P$. euphratica wurde im Verlauf der Vegetationsperiode eine starke Salzanreicherung in den Blättern festgestellt. $C$. caput-medusae hatte die geringsten Salzgehalte der untersuchten Arten und wies nur zum Ende der Vegetationsperiode einen Anstieg im Natriumgehalt auf. $A$. sparsifolia zeigte die stärkste Regulation bei der lonenaufnahme und reicherte vor allem Calcium an (ARNDT et al. 2002). Bei starker Salzanreicherung in der Vakuole können als Ausgleich im Cytoplasma verstärkt kompatible Osmotika auftreten. Bei A. sparsifolia und T. ramosissima wurde dies durch den zyklischen Zuckeralkohol Pinitol erreicht, bei C. caput-medusae von chiro-Inositol. Bei $P$. euphratica könnten myo-Inositol oder Zucker eine Rolle spielen (ARNDT et al. 2002). Insgesamt sind aber die Gehalte an Osmotika eher niedrig.

Tamarix ramosissima hatte hohe Salzgehalte in den photosynthetisch aktiven Kurztrieben und Blättern, die sich aber im Jahresverlauf wenig veränderten (ARNDT et al. 2002). T. ramosissima zeigte keine besondere Regulation bei der Ionenaufnahme, negative Effekte der Salzanreicherung wurden durch die Ausscheidung der Salze über Salzdrüsen vermieden (WALLACE \& KLEINKOPF 1974). Die Mittagswasserpotentiale nahmen bei allen untersuchten Arten im Laufe der Vegetationsperiode leicht ab, was vielleicht zu einem Teil mit der Anreicherung osmotisch wirksamer Substanzen erklärt werden kann. Einen weiteren Hinweis auf die Anreicherung von Osmotika lieferten die Ergebnisse der PV-Kurven: vor allem $P$. euphratica, aber auch $T$. ramosissima zeigten einen Anstieg des osmotischen Potentials im Laufe der Vegetationsperiode (THOMAS, F.M., unveröffentlicht). 


\subsubsection{Transpiration und stomatäre Leitfähigkeit}

In Trockengebieten und schon bei angespanntem Bodenwasserhaushalt und starker Transpirationsbelastung in gemäßigten Breiten treten mittags Einschränkungen der Transpiration und bereits morgens eine starke Abnahme der stomatären Leitfähigkeit auf (z.B. BACKES 1996, LÖSCH 2001). Die Stomata regeln bei verändertem Wasser-angebot und Wasserverbrauch die Transpiration (z.B. HuBbARD, BOND \& RYAN 1999, SPERRY et al. 2002). Damit wird ein Absenken der Wasserpotentiale in einen kritischen Bereich vermieden. Bei dieser Reduktion des Wasserverbrauch muss jedoch eine Verringerung der Assimilation in Kauf genommen werden.

Die Transpirationstagesgänge der untersuchten Pflanzen wiesen keine Mittagsdepression auf, obwohl an den meisten Tagen ein sehr hohes VPD herrschte (4$6 \mathrm{kPa}$ am Nachmittag) und somit die Transpirationsbeanspruchung hoch war. Wenn die Blatttemperaturen stark ansteigen, wird für Pflanzen der Schutz vor Überhitzung wichtig, da schon ab Temperaturen von $40{ }^{\circ} \mathrm{C}$ manche Enzyme der Photosysteme gehemmt werden (z.B. SCHOPFER \& BRENNICKE 1999). Kühlung durch Konvektion tritt dann auf, wenn die Blattemperatur höher als die Lufttemperatur ist, und wird bedeutend, wenn die Windgeschwindigkeit ansteigt. Bei den untersuchten Arten waren die gemessenen Blattemperaturen fast immer niedriger oder gleich der Lufttemperatur. Bei Lufttemperaturen von mehr als $40{ }^{\circ} \mathrm{C}$, wie sie im Sommer im Untersuchungsgebiet auftreten, kann die Kühlung durch Transpiration wichtig sein. Bei $A$. sparsifolia traten nachmittags Blatttemperaturen von mehr als $40{ }^{\circ} \mathrm{C}$ bei den Messungen im Juni und Juli 1999 sowie im Mai, August und September 2000 auf, bei C. caput-medusae im Juli 1999 sowie Juni und August 2000 und bei T. ramosissima im Mai 1999. Nur P. euphratica hatte keine Blatttemperaturen in dieser Höhe, allerdings konnte bei den Pappeln auch nicht in der oberen Krone gemessen werden, wo die Temperaturen am höchsten sind, sondern nur in einem Kronenbereich, wo zumindest eine Zeit des Tages Schatten fällt. Der Wasserverlust durch Transpiration wird in Kauf genommen, um die Blatttemperaturen unter einem kritischen Schwellenwert zu halten. Die Transpiration muss also hoch genug sein, damit eine Kühlung gewährleistet ist; 
andererseits muss sie niedrig genug sein, um zu starken Wasserverlust zu vermeiden.

Die stomatäre Leitfähigkeit folgte nicht wie die Transpiration der Strahlung, sondern nahm vom Morgen zum Nachmittag hin ab oder blieb den ganzen Tag auf einem sehr niedrigen Niveau. So wurde offenbar ein Absinken der Blattwasserpotentiale unter einen kritischen Schwellenwert, bei dem z.B. Kavitationen auftreten können, vermieden. Man konnte beobachten, dass entweder die Leitfähigkeit bei abnehmendem Wasserpotential konstant gehalten wurde (C. caput-medusae, Abb. 4.4.2 und 4.5.6), also noch kein kritischer Bereich des Wasserpotentials erreicht wurde, bei dem eine Einschränkung der stomatären Leitfähigkeit erforderlich wäre, oder dass sie mit steigender Leitfähigkeit die Blattwasserpotentiale absanken ( $P$. euphratica) und sich bei sinkender Leitfähigkeit erholten (Abb. 4.4.4 und 4.5.13). GRIES et al. (2002b) konnten bei Populus euphratica und Tamarix ramosissima einen engen Zusammenhang zwischen Mittagswasserpotential und Mittagsleitfähigkeit (zwischen 14:00 und 17:00 Uhr) auf nahe gelegenen Untersuchungsflächen feststellen. Bei den in der vorliegenden Arbeit untersuchten Flächen konnte dagegen nur bei $T$. ramosissima eine schwache Korrelationen gefunden werden $\left(r^{2}=0,375 ; P=0,014\right)$. Auch für die Beziehung der gesamten Tagesgänge der Wasserpotentials zur stomatären Leitfähigkeit der untersuchten Arten fand sich kein solcher Zusammenhang.

Bei zunehmendem VPD im Tagesverlauf kann man eine abnehmende stomatäre Leitfähigkeit erwarten, wenn die Wasserverfügbarkeit der Pflanzen eingeschränkt ist und ein Absinken des Wasserpotentials vermieden werden soll (SPERRY \& POCKMAN 1993, LösCH 2001). Bei T. ramosissima konnte man nur im Mai 1999 eine Beziehung zwischen stomatärer Leitfähigkeit und VPD ermitteln $\left(r^{2}=0.791\right.$, $P=0.0013)$. Im weiteren Jahresverlauf war die stomatäre Leitfähigkeit deutlich niedriger als im Frühjahr (Abb. 4.5.10) und änderte sich weder mit sinkendem Wasserpotential noch mit steigendem VPD. OREN et al. (1999) beschreiben, dass eine niedrigere stomatäre Leitfähigkeit oft mit geringerer Sensitivität gegenüber dem VPD einhergeht. Dies könnte auch für T. ramosissima zutreffen.

Der Einfluss des VPD auf die stomatäre Leitfähigkeit war bei C. caput-medusae signifikant $\left(r^{2}=0.274, P=0.001\right)$, ebenfalls bei $A$. sparsifolia im Sommer $\left(r^{2}=\right.$ $0.369, P=0.0016)$. Bei $P$. euphratica konnten nur Zusammenhänge gefunden 
werden, wenn man die morgendlichen Werte aus der Betrachtung heraus lässt, da morgens zwar das niedrigste VPD, aber nicht immer die höchste stomatäre Leitfähigkeit des Tages gemessen wurde. Dies liegt wahrscheinlich daran, dass die Beleuchtungsstärke noch nicht ausreichend bzw. die blattinterne $\mathrm{CO}_{2}-K o n z e n-$ tration noch nicht weit genug abgesunken war, um volle Spaltenöffnung zu induzieren. Im weiteren Tagesverlauf wird die Höhe der stomatären Leitfähigkeit von der Höhe des VPD beeinflusst $\left(r^{2}=0,668\right.$ bis 0,746$)$.

Im Sommer ist die stomatäre Leitfähigkeit bei den untersuchten Arten (außer bei $P$. euphratica) zwar am Nachmittag deutlich geringer als im Frühjahr, sie stimmt aber nicht immer klar mit dem Tagesverlauf des Wasserpotentials oder des VPD überein.

Betrachtet man die Tagesgänge der stomatären Leitfähigkeit an den verschiedenen Messterminen, so wird deutlich, dass die Pflanzen ihren Wasserverbrauch im Verlauf der Vegetationsperiode zunehmend einschränken. Nur $P$. euphratica zeigte eine im Verlauf der Vegetationsperiode zunehmende Leitfähigkeit.

Bei vielen Pflanzen sinkt die stomatäre Leitfähigkeit mit zunehmendem Blattalter ab (LöSCH 2001). Auch dies kann den Jahresverlauf der stomatären Leitfähigkeit bei den untersuchten Arten, mit Ausnahme von $P$. euphratica, erklären.

Die sehr niedrige stomatäre Leitfähigkeit bei $T$. ramosissima und $P$. euphratica im September (Abb. 4.5.10 und 4.5.14) kann vermutlich auf beginnende Seneszenz zurückgeführt werden. Zu dieser Jahreszeit waren außerdem alle Pappelblätter durch Fraß geschädigt.

Im Artvergleich zeigt Populus euphratica die höchste stomatäre Leitfähigkeit und die höchste Transpirationsrate der untersuchten Arten, Calligonum caput-medusae die niedrigsten Werte. Alhagi sparsifolia und Tamarix ramosissima zeigen eine ähnlich hohe Transpiration, aber A. sparsifolia hat eine höhere Leitfähigkeit.

Anhand der Transpirations- und Leitfähigkeitsmessungen kann man für alle untersuchten Arten feststellen, dass sie keinem starken Wasserstress ausgesetzt sind. Sie verbrauchen viel Wasser, zeigen keine Mittagsdepression des Gaswechsels und werden nur teilweise von mikroklimatischen Faktoren eingeschränkt. 
Diese Ergebnisse zeigen deutlich, dass die untersuchten Arten Grundwasseranschluss haben müssen.

\subsubsection{Effekte der experimentellen Bewässerung auf den Wasserhaushalt der Pflanzen}

Obwohl alle untersuchten Arten Zugang zum Grundwasser hatten, bestand die Möglichkeit, dass die Bestände von der Bewässerung profitieren könnten. Ein Vergleich der Wasserhaushaltsparameter auf den bewässerten Flächen vor und nach der Bewässerung sowie ein Vergleich zwischen bewässerten und unbewässerten Flächen soll darüber Aufschluss geben.

Wenn die Pflanzen von der Bewässerung profitieren könnten, würden die Wasserpotentiale ansteigen (BRÉDA, GRANIER \& AusSENAC 1995, DAMESIN, RAMBAL \& JOFFRE 1997), ebenso die Saftfluss- und Transpirationsraten (DAVID et al. 1997) sowie die stomatäre Leitfähigkeit (HINCKLEY et al. 1980, COMSTOCK 2002).

Die $\delta^{13}$ C-Werte nehmen nach Bewässerung ab (LEFFLER \& EVANS 2001), wenn man davon ausgeht, dass die stomatäre Leitfähigkeit vor einer Bewässerung wegen Wassermangel eingeschränkt und somit die interne $\mathrm{CO}_{2}$-Konzentration im Blatt niedrig war.

\subsubsection{Vergleich der bewässerten ungenutzten Flächen vor und nach der Bewässerung}

Zunächst soll auf Auswirkungen der Bewässerung auf den Wasserhaushalt der untersuchten Pflanzen auf den bewässerten Flächen (FN) eingegangen werden.

Nach der experimentellen Bewässerung konnte kein signifikanter Bewässerungseffekt im Blattwasserpotential der untersuchten Arten festgestellt werden. Bei $A$. sparsifolia liegt keine Messung der Wasserpotentiale in den Wochen direkt vor der Bewässerung vor. Das predawn-Wasserpotential im Mai lag signifikant höher als der Wert im Juli nach der Überflutung, auch die Mittagswasserpotentiale waren im Mai höher. Daraus kann aber nicht gefolgert werden, dass die Bewässerung die 
Wasserversorgung verschlechtert hat. Vielmehr dürfte der Bewässerungseffekt zu vernachlässigen sein und das Absinken des Wasserpotentials einem saisonalen Trend folgen, der durch den im Lauf der Vegetationsperiode steigenden Wasserverbrauch und dem Witterungseinfluss hervorgerufen wird. Nur bei $C$. caput-medusae wurden nach der Bewässerung leicht höhere Mittagswasserpotentiale gemessen als davor, dieser Unterschied ist aber nicht signifikant. Bei $T$. ramosissima und $P$. euphratica fand die Bewässerung Anfang August statt. Das predawn-Wasserpotential lag an den Messterminen im August tendenziell höher als vor der Bewässerung (Abb. 4.4.5), allerdings nicht signifikant höher. Auch bei $A$. sparsifolia wurden im August etwas höhere predawn-Potentiale gemessen als im Juli. In diesem Fall kann aber ein Bewässerungseffekt ausgeschlossen werden, da die Bewässerung auf der Alhagi-Fläche schon Ende Juni stattfand. Es kann also angenommen werden, dass bei $A$. sparsifolia ebenso wie bei $T$. ramosissima und $P$. euphratica andere Faktoren eine Rolle spielen. Bei A. sparsifolia und $P$. euphratica sind Z.B. am Tag vor der Messung und am Messtag im August sowohl das VPD, als auch die Temperatur und die Strahlungssumme niedriger als im Juli. Dies führte offensichtlich zu einem leicht höheren predawn-Wasserpotential als im Juli.

Die Transpiration erreichte bei $C$. caput-medusae und $P$. euphratica bei einem vergleichbaren VPD ähnliche Werte vor und nach der Bewässerung. Bei $A$. sparsifolia und $T$. ramosissima war sie danach höher. Dies ist bei $T$. ramosissima vermutlich auf ein höheres VPD am Messtag nach der Bewässerung zurückzuführen, bei $A$. sparsifolia war jedoch an beiden Tagen das VPD ähnlich hoch. Da die letzten Messungen von Transpiration, stomatärer Leitfähigkeit und Wasserpotential nicht direkt vor der Bewässerung durchgeführt werden konnten, können saisonale Unterschiede zwischen den beiden Messterminen nicht ausgeschlossen werden. Im Untersuchungsjahr 2000 stiegen z.B. die Transpirationsraten zwischen Juli und August bei $A$. sparsifolia und $P$. euphratica an. In einer vergleichbaren Untersuchung an $T$. ramosissima und $P$. euphratica gab es ebenfalls eine saisonale Zunahme der Transpiration zwischen Juli und August (GRIES et al. 2003). Daher kann man anhand dieser Daten nicht auf Bewässerungseffekte schließen. Zudem gab es bei der Messung im Juli, bei $C$. caput-medusae, $T$. ramosissima und $P$. euphratica die letzte Messung vor der 
Bewässerung, technische Schwierigkeiten bei den Porometern. Es ist daher nicht klar, ob die im Juli 1999 gemessene Mittagsdepression nicht auf Messfehler zurückgeht. Der Saftfluss erfuhr an den gleichen Tagen keine Einschränkung am Mittag.

Bei der Saftflussmenge, die an klimatisch ähnlichen Tagen vor und nach der experimentellen Bewässerung verglichen wurde (Anhang Tab. 11), konnten keine signifikanten Unterschiede festgestellt werden.

Die $\delta^{13} \mathrm{C}$-Werte waren nur bei $A$. sparsifolia signifikant höher vor der Überflutung als danach. Bei allen untersuchten Arten war, wie am Jahresgang der Isotopensignatur zu sehen (Abb. 4.8.1), der Wert Ende Juni höher als Ende Juli. Während bei $C$. caput-medusae die Bewässerung erst Ende Juli und bei $T$. ramosissima und $P$. euphratica Anfang August stattfand, als die Isotopensignatur schon einen relativ konstanten Wert erreicht hatte (siehe Kapitel 5.4.5), wurde bei A. sparsifolia die Bewässerung schon Ende Juni durchgeführt. Die jahreszeitliche Veränderung überlagerte also einen möglichen Bewässerungseffekt.

Zusätzlich kann man die osmotischen Potentiale, die aus Druck-Volumen-Kurven berechnet wurden (THOMAS, F.M. unveröffentlicht), heranziehen. Bei einer besseren Wasserversorgung der Pflanzen würde man nach der Bewässerung ein Ansteigen der osmotischen Potentiale erwarten. Die osmotischen Potentiale stiegen nur bei $T$. ramosissima an; dieser Anstieg stimmte aber nicht mit dem Verlauf der Wasserpotentiale überein.

Da die untersuchten Arten ihr Wachstum zum Zeitpunkt der Bewässerung schon abgeschlossen hatten (Abb. 4.3.1), konnte im Untersuchungsjahr 1999 kein Effekt auf das Wachstum mehr erwartet werden. Ein durch die Bewässerung entstandener Wasservorrat im Boden hätte im folgenden Jahr zu einem besseren Wachstum beitragen können. Die Bodenwassergehalte waren aber am Ende der Vegetationsperiode 1999 schon wieder genauso niedrig wie vor der Bewässerung (Abb. 4.2.1). Auch im Folgejahr wurde auf den bewässerten Flächen kein verbessertes Wachstum festgestellt. Insgesamt war die Produktion der untersuchten Arten im Jahr 2000 niedriger als 1999. Vor allem auf der bewässerten AlhagiFläche (AF) wurde 2000 ein deutlich schlechteres Wachstum festgestellt, das 
vermutlich auf Konkurrenzeffekte im Vorjahr zurückzuführen ist. Im Anschluss an die Bewässerung 1999 wuchsen begünstigt durch das höhere Wasserangebot zahlreiche Chenopodiaceen (vor allem Halogeton arachnoideus Moq. in DC. und Salsola paulsenii Litw.) aus der Samenbank auf der Fläche auf (THOMAS et al. 2001), die A. sparsifolia überwuchsen. A. sparsifolia konnte dadurch weniger Reservestoffe bilden und trieb im Folgejahr an vielen Stellen nicht mehr aus.

Zusammenfassend kann man feststellen, dass die experimentelle Bewässerung keine Auswirkungen auf den Wasserhaushalt der untersuchten Arten hatte. Auch auf die Produktivität im Folgejahr wurde kein positiver Effekt gemessen, sondern bei Alhagi sparsifolia sogar ein negativer Effekt.

\subsubsection{Vergleich der bewässerten (FN) und unbewässerten (DN) Flächen}

Es war zunächst erwartet worden, dass die Pflanzen auf den unbewässerten Flächen (DN) größerem Trockenstress ausgesetzt waren als auf den bewässerten Flächen (FN). Auch nachdem sich herausgestellt hatte, dass alle Pflanzen das Grundwasser erreichen, war zu prüfen, ob sich die Wasserverfügbarkeit zwischen den Teilflächen unterschied.

Auf den bewässerten Flächen wurden in den Monaten nach der Bewässerung eine höhere Transpiration und eine höhere stomatäre Leitfähigkeit als auf den unbewässerten Flächen im gleichen Monat gemessen. Da die Messungen auf den Kontrollflächen (DN) nicht an den gleichen Tagen stattfanden wie auf den bewässerten Flächen (FN), wurden VPD, Strahlungssumme und Tagesmitteltemperatur der jeweiligen Messtage eines Monats verglichen. Die Unterschiede im Wasserhaushalt ließen sich nicht mit den mikroklimatischen Parametern vereinbaren. Der Wasserverbrauch war auf den unbewässerten Flächen geringer, unabhängig davon, ob die Lufttemperatur und das VPD an den Messtagen höher oder niedriger war. Daher muss von einer besseren Wasserversorgung auf den bewässerten Flächen ausgegangen werden. Eine Ausnahme bildeten die Calligonum-Flächen (CF und CD) Ende September 1999: an diesem Termin waren die Transpirationsraten gleich und die Leitfähigkeit auf der unbewässerten Fläche (CD) höher. 
Im Falle von C. caput-medusae und $T$. ramosissima lagen die nicht bewässerten Flächen auf Dünen, weil sie nur so von der Bewässerung ausgespart werden konnten. Aus dem größeren Abstand zum Grundwasser ergeben sich längere Leitungswege. Deswegen kann man geringere Wasserpotentiale erwarten, analog zu den Unterschieden im Wasserpotential sowie in stomatärer und hydraulischer Leitfähigkeit mit zunehmender Baumhöhe (SCHÄFER, OREN \& TENHUNEN 2000). Weil die stomatäre Leitfähigkeit und das Blattwasserpotential gekoppelt sind, wird bei niedrigeren Wasserpotentialen auch eine geringere Leitfähigkeit auftreten. Dies wurde schon bei einem Höhengradienten von Dünen mit zunehmender Entfernung zum Grundwasser für Populus euphratica und Tamarix ramosissima festgestellt (GRIES et al. 2003). Die niedrigere stomatäre Leitfähigkeit und die geringeren Transpirationsraten auf den CD- und TD-Flächen sowie die höheren $\delta^{13} \mathrm{C}$-Werte (auf der TD-Fläche) lassen sich somit vermutlich auf einen längeren Leitungsweg zurückzuführen.

Bei $P$. euphratica müsste aufgrund der höheren stomatären Leitfähigkeit und Transpiration des bewässerten Bestandes von einer Verbesserung der Wasserversorgung durch die Bewässerung ausgegangen werden, allerdings gibt es nur eine Vergleichsmessung des unbewässerten Bestandes nach der Bewässerung. Es ist also möglich, dass die Wasserversorgung der beiden Bestände prinzipiell unterschiedlich ist. Das in Jahrringanalysen gezeigte durchgängig schlechtere Wachstum des Kontrollbestandes während seiner ganzen 22-jährigen Entwicklung unterstützt diese Annahme (GRIES, D., unveröffentlicht). Von den Bäumen auf der bewässerten Fläche ist aus molekularbiologischen Untersuchungen mittels AFLP-Analysen bekannt, dass sie ein Klon sind (BRUELHEIDE, H., unveröffentlicht). Es könnte sein, dass die unbewässerte Fläche von einem weniger vitalen Klon bestanden ist. Auch A. sparsifolia ist eine Art mit ausgeprägtem klonalen Wachstum. Aus Untersuchungen im Oasenvorland von Qira ist bekannt, dass sich verschiedene voneinander abgegrenzte Gruppen von Alhagi sparsifolia bezüglich ihrer $\delta^{15} \mathrm{~N}$-Signatur unterscheiden (ARNDT, S.K., unveröffentlicht). Diese Pflanzengruppen unterscheiden sich in ihrer Physiologie und sind vermutlich durch vegetative Vermehrung aus verschiedenen Individuen entstanden. Auch im Falle von A. sparsifolia könnte also ein anderer Klon auf der unbewässerten Fläche die Erklärung für den unter-schiedlichen Wasserverbrauch 
der Pflanzen auf den beiden Untersuchungsflächen sein. Die molekularbiologischen Untersuchungen von Blattmaterial stehen jedoch noch aus.

Bei Alhagi könnten höhere Transpirationsraten auf der AFN-Fläche auch durch eine geringere Anzahl Büsche pro Fläche bedingt sein. Bei einer gegebenen unterirdischen hydraulischen Leitfähigkeit kann man bei klonalem Wachstum davon ausgehen, dass eine geringere Buschdichte höhere Transpirationsraten der Einzelbüsche erlaubt. Dies wird unterstützt von der Feststellung, dass das Wachstum der Einzelbüsche umso besser ist, je geringer die Bestandesdichte ist (GRIES, D., unveröffentlicht). Auch dieser Befund kann bei der vorliegenden Bestandesdichte und der hohen Strahlungsintensität nicht mit Beschattung, sondern nur mit kloninterner Wasserkonkurrenz erklärt werden.

Da es keine Messungen von den unbewässerten Flächen im Frühsommer 1999 gibt, kann man nicht endgültig klären, ob ein genereller Unterschied im Wasserhaushalt zwischen den Flächen auch schon vor der experimentellen Bewässerung bestanden hat. Die Transpirations- und Leitfähigkeitsmessungen sind also insgesamt nicht umfassend genug, um die Frage eines positiven Bewässerungseffekts zu klären.

Die $\delta^{13} \mathrm{C}$-Werte auf den unbewässerten Flächen zeigten einen vergleichbaren Jahresverlauf wie die auf den bewässerten Flächen (Abb. 4.8.1). Die Werte auf den unbewässerten liegen eher etwas höher als die auf den bewässerten Flächen, aber nur bei $T$. ramosissima sind sie während der gesamten Vegetationsperiode signifikant höher als die Werte auf der bewässerten Fläche, was ebenso wie die geringere stomatäre Leitfähigkeit auf eine größere Entfernung zum Grundwasser zurückgeführt werden kann. Bei A. sparsifolia wurden im Untersuchungsjahr 2000 ähnliche Isotopensignaturen wie schon 1999 gemessen. Daran kann man sehen, dass es auch keinen lang anhaltenden Bewässerungseffekt gab, der erst im Folgejahr seine Auswirkungen zeigt.

Aufgrund der Annahme, dass ein Bodenwasservorrat aufgebaut wird, hätte man im folgenden Jahr eine höhere Produktivität der bewässerten Bestände erwarten können. Tatsächlich war die Produktivität aber im Untersuchungsjahr 2000 insgesamt geringer als 1999. Die Pflanzen auf den bewässerten Flächen zeigten 
auch 2000 kein besseres Wachstum als die Pflanzen auf den Kontrollflächen (GRIES et al. 2002).

\subsection{Wassernutzungseffizienz (WUE)}

\subsubsection{Wasserverbrauch der Arten berechnet aus Saftflussmessungen}

Ursprünglich waren niedrige Saftllussraten und vor allem Einschränkungen des Saftflusses am Mittag erwarten worden, weil von einem Wasserdefizit der untersuchten Arten ausgegangen worden war. Da aber alle Pflanzen Grundwasseranschluss haben, konnte man vermuten, dass kein starker Trockenstress auftreten und vergleichbare Saftflussraten wie in anderen Trockengebieten mit ausreichender Wasserzufuhr gemessen würden.

Im Frühjahr 1999 wurden bei $A$. sparsifolia und $T$. ramosissima sehr hohe trockengewichtsbezogene Transpirationsraten und eine hohe stomatäre Leitfähigkeit gemessen, von C. caput-medusae liegt in diesem Zeitraum keine Messung vor. Im Jahr 2000 wurden ebenfalls die höchste stomatäre Leitfähigkeit und Transpiration im Frühjahr bei noch jungen Trieben und Blättern gemessen. Auch die blattspezifische hydraulische Leitfähigkeit ist im Frühjahr bei $A$. sparsifolia und $C$. caput-medusae höher als im Sommer (THOMAS, F.M., unveröffentlicht). Vergleicht man die Verläufe mit der Entwicklung der Blattflächen, kann man erkennen, dass zur Zeit der schnellsten Blattentwicklung die höchste hydraulische Leitfähigkeit auftritt.

Im Frühjahr treten bei $A$. sparsifolia und $C$. caput-medusae auch die höchsten trockengewichtsbezogenen Saftflussraten auf (Abb. 4.6.4). Die noch jungen Blätter und Kurztriebe sind noch wenig gegen den Wasserverlust geschützt ( $\mathrm{LÖSCH} 2001$ ). Bei $T$. ramosissima war die Modellierung der Frühjahrswerte schwierig, da tagsüber geringere Saftflussraten gemessen wurden als nachts. Es wurden nur geringe trockengewichtsbezogene Saftflussraten ermittelt.

Die geringeren Saftlussraten per Trockengewicht sind im weiteren Verlauf des Jahres zum Teil durch den Anstieg des spezifischen Blattgewichtes zu erklären, 
vor allem aber durch den Verlauf der Blattentwicklung (Abb. 4.3.1) Im Frühjahr steigen die absoluten Saftflussmengen zunächst schneller an als die Blattmasse, zum Sommer hin nimmt der absolute Wasserverbrauch pro Trieb sogar leicht ab, während die Blattmasse konstant bleibt (als Beispiel siehe A. sparsifolia Abb. 4.6.3).

Die Saftlussmessungen ergaben in der vorliegenden Arbeit Werte in einer ähnlichen Größenordnung wie in den Angaben anderer Autoren. Aufgrund unterschiedlicher Bezugsgrößen kann man jedoch die Saftflusswerte oft nicht direkt vergleichen.

Die transpirierten Wassermengen waren bei Tamarix ramosissima zum Teil niedriger als der in anderen Gebieten gemessene Wasserverbrauch der gleichen Art. In Nevada (USA) wurden in einem Tamarix-Bestand Saftflusssummen von 1,2 bis fast $6 \mathrm{~kg} \mathrm{H}_{2} \mathrm{O}^{*} \mathrm{~m}^{-2}$ Blattfläche ${ }^{*} \mathrm{Tag}^{-1}$ am Übergang von einem Flussbett zur Wüste gemessen. Die niedrigeren Werte stammen aus einer Trockenperiode. Näher am Fluss wurden sogar bis zu $21 \mathrm{~kg} \mathrm{H}_{2} \mathrm{O}^{*} \mathrm{~m}^{-2}$ Blattfläche${ }^{*} \mathrm{Tag}^{-1}$ erreicht (DEVITT et al. 1997a). Die Werte der Trockenperiode entsprachen etwa den höchsten Werten $\left(1,2 \mathrm{~kg} \mathrm{H}_{2} \mathrm{O}^{*} \mathrm{~m}^{-2}\right.$ Blattfläche $\left.{ }^{*} \mathrm{Tag}^{-1}\right)$ in der vorliegenden Untersuchung.

Im Oasenvorland von Qira wurden ähnliche Werte ermittelt wie bei Stecklingen von T. ramosissima aus der Sonoran Desert, die im Gewächshaus einen Wasserverbrauch von $12,86 \pm 0,67 \mathrm{~g} \mathrm{H}_{2} \mathrm{O}^{*} \mathrm{~g} \mathrm{TG}^{-1 *} \mathrm{Tag}^{-1}$ zeigten (GLENN et al. 1998). In wie weit man diese Angaben wirklich vergleichen kann, bleibt unklar, da die meisten Autoren nicht angeben, mit welcher Methode sie die Blattfläche von Tamarix bestimmt haben. Da bei Tamarix sicherlich nicht der Wasserverbrauch der stark reduzierten Blättchen, sondern des gesamten Kurztriebes gemessen wurde, ist die Frage der Bezugsgröße wichtig, aber meist nicht nachvollziehbar.

\subsubsection{Vergleich von Transpiration und Saftfluss}

Vergleicht man Berechnungen zum Tageswasserverbrauch auf Basis des Saftflusses und der Transpiration, so wurde häufig beobachtet, dass die auf die gesamte Pflanze hochgerechneten Transpirationsmessungen höhere Werte als die 
Saftflussmessungen liefern (ANSLEY et al. 1994, BACKES 1996). Da die Transpiration normalerweise an Blättern in der Sonnenkrone gemessen wird, kann man davon ausgehen, dass die hieraus hochgerechneten Werte die Transpiration der gesamten Krone überschätzen. Für Schattenblätter oder ganz allgemein Blätter im Kroneninneren wurden oft niedrigere Transpirationsraten gemessen, da innerhalb der Krone ein geringeres VPD und eine geringere Strahlung herrschen. BACKES (1996) beschreibt, dass an Tagen mit hohem VPD die Saftflussraten anstiegen, während die Transpirationsraten eher gleich blieben. Die Werte für den Tageswasserverbrauch berechnet aus Saftfluss- und Transpirationsmessungen glichen sich bei hohem VPD an, weil dann auch im Kroneninnenraum die Transpirationsraten anstiegen, obwohl sich die Strahlungsverhältnisse in der Krone nicht änderten (BACKES 1996).

Bei den in der vorliegenden Arbeit untersuchten Arten A. sparsifolia, C. caputmedusae und $T$. ramosissima liegen aber die am gleichen Tag gemessenen Saftflusssummen an fast allen Terminen höher als der Wasserverbrauch durch Transpiration. Da im Untersuchungsgebiet an den meisten Tagen ein sehr hohes VPD herrschte und die Kronenstruktur der untersuchten Arten eher locker ist, könnte man erwarten, dass Saftfluss- und Transpirationsmessungen einen ähnlich hohen Wert liefern, weil die Unterschiede zwischen äußerer und innerer Krone gering bleiben und damit die Transpirationsmessungen nicht den Wasserverbrauch überschätzen. Dass aber die Saftflussmessungen sogar höhere Werte liefern als die Transpirationsmessungen, kann daran liegen, dass die Transpiration der jungen, aber schon verholzten Triebe zu einem beträchtlichen Teil zum Wasserverbrauch beiträgt. Dieser Teil der Transpiration wird nur von den Saftlusssystemen, nicht aber von den Porometermessungen erfasst und kann so $z u$ einem höheren Wasserverbrauch berechnet aus den Saftflussmessungen führen.

Dass der Saftfluss sehr eng mit der Strahlung korreliert, ist aus verschiedenen Untersuchungen bekannt (z.B. ALLEN \& GRIME 1995) und wurde auch in der vorliegenden Arbeit bestätigt. Die Modellierung des Saftflusses bei drei der untersuchten Arten erfolgte sogar mit der Globalstrahlung.

Bei $P$. euphratica wurden sehr niedrige Saftflusswerte im Vergleich mit den Transpirationswerten errechnet (Abb. 4.6.9 und 4.6.10). Man könnte, wie oben be- 
schrieben, eine Überschätzung des Wasserverbrauchs aus den Transpirationsmessungen vermuten. In diesem Fall erscheinen aber die Saftflussraten unterschätzt worden zu sein. So wurde bei einer Studie in Israel z.B. bei Saftlussmessungen an Eucalyptus ("heat-pulse“-System) ein Wasserverbrauch von $30-120 \mathrm{~kg}$ am Tag gemessen, auf einer Fläche, auf der die Bäume neben einer Bewässerung im Sommer auch Zugang zum Grundwasser hatten (COHEN et al. 1997). Die Bäume hatten mit durchschnittlich $5 \mathrm{~m}$ eine mit den untersuchten Pappeln vergleichbare Höhe, der LAl des Bestandes war mit $1,5 \mathrm{~m}^{2 *} \mathrm{~m}^{-2}$ sogar geringer. Bei Populus euphratica wurde dagegen nur ein Wasserverbrauch von etwa $1 \mathrm{~kg}$ am Tag aus Saftflussmessungen berechnet. Nur im Frühjahr wurden bei einem Baum auch Werte von ca. 2-6 kg Wasser am Tag gemessen.

Es kann bei Verwendung des GrANIER-Systems bei Bäumen mit einem schmalen Leitgewebe zu einer Unterschätzung des Saftflusses kommen (KöSTNER, GRANIER \& CERMÁK 1998). Dies ist vermutlich auf eine schlechtere Wärmeleitungskapazität außerhalb des Leitgewebes zurückzuführen. Außerdem kann ein Temperaturgradient die Messung beeinflussen, der durch starke Sonneneinstrahlung oder Wärmespeicherung im Stamm zustande kommt. Dies wirkt sich in einer fehlerhaften Berechnung des nächtlichen Nullpunktes aus.

Mögliche Fehlerquellen bei der Saftflussmessung an Populus euphratica sind eine Unterschätzung der Saftflussfläche durch unvollständige Anfärbung des Holzes, die Korrosion der Sensoren und ein schlechter Kontakt der Sensoren zum Holz. Es fällt auf, dass die frisch mit Sensoren bestückten Bäume im August 1999 etwa dreibis zehnmal so hohe Saftlussraten zeigten wie die beiden Bäume, an denen durchgängig gemessen wurde (Abb. 4.6.5). In ihrer Biomasse unterschieden sich die ausgewählten Bäume nicht so stark, dass dieser Unterschied im Wasserverbrauch zu erklären wäre. Vermutlich war der Kontakt der dauerhaft laufenden Sensoren zum Holz nicht mehr optimal. Es trat mehrmals das Problem auf, dass Sensoren durch Korrosion spröde wurden und brachen. Populus euphratica ist bekannt für die hohen Gehalte an Salzen im Xylemsaft, die sogar als Salzquelle genutzt werden (LIU JIAJIN, unveröffentlicht; PAN \& YING 1995, WANG et al. 1996). Populus euphratica ist in der Lage, bis zu einem gewissen Grad Salz aus den Blättern auszuschließen (MA et al. 1996), akkumuliert aber trotzdem im Jahresverlauf große Mengen Salz in den Blättern. Der Gehalt an $\mathrm{NaCl}$ in den 
Blättern stieg von $63 \mathrm{mmol}^{*} \mathrm{~kg} \mathrm{TG}^{-1}$ im Mai auf $313 \mathrm{mmol}^{*} \mathrm{~kg} \mathrm{TG}^{-1}$ im September (ARNDT et al. 2002). Man kann also davon ausgehen, dass der Xylemsaft sehr salzhaltig ist, und sollte für $P$. euphratica daher ein Saftflusssystem verwenden, dass nicht korrosionsanfällig ist.

Beträchtliche Fehler können außerdem bei der Hochrechnung vom Sensor auf den gesamten Baum auftreten, da die Variation des Saftflusses innerhalb des Stammes groß sein kann (KöSTNER, GRANIER \& CERMÁK 1998).

Eine andere Hochrechnung des Wasserverbrauchs auf die gesamte Vegetationsperiode erfolgte über die mikrometeorologischen Daten der Wetterstationen und die stomatäre Leitfähigkeit mit dem PENMAN-MONTEITH-Ansatz (VAN HYLCKAMA 1980, MONTEITH \& UNSWORTH 1990). Diese Berechnung führte zu einem weiteren Wert für den flächenbezogenen Wasserverbrauch der untersuchten Arten (THOMAS et al., in Vorbereitung). Berechnet man den Wasserverbrauch über die Vegetationsperiode mit dem PENMAN-MonTEITH-Ansatz, findet man bei $P$. euphratica sehr viel höhere Werte als bei den Saftflussmessungen (Tab. 6). Auch diese Berechnungen bestätigen, dass die Saftflussmessungen bei $P$. euphratica keine glaubwürdigen Messwerte lieferten. Dagegen fanden z.B. DAVID et al. (1997) eine sehr gute Übereinstimmung der mit dem GRANIER-System gemessenen Saftflusswerte und den mit PENMAN-MONTEITH berechneten Werten bei Eucalyptus. Bei den anderen untersuchten Arten lag der mit beiden Methoden berechnete Wasserverbrauch in einer vergleichbaren Größenordnung (siehe Tab. 6), wobei der mit dem PENMAN-MONTEITH berechnete Wasserverbrauch niedriger als der aus den Saftflussmessungen ermittelte Verbrauch war. Der deutlich höhere Wasserverbrauch aus Saftflussmessungen bei $T$. ramosissima ist vermutlich darauf zurückzuführen, dass der Saftfluss innerhalb eines geschlossenen Bestandes gemessen wurde, die mikroklimatischen Parameter, die in die PENMANMONTEITH-Berechnung eingehen, jedoch auf einem vegetationsfreien Teil der Untersuchungsfläche. 
Tab. 6: Wasserverbrauch der untersuchten Arten 1999.

Der Wasserverbrauch wurde berechnet auf Basis der Saftflusssumme der Vegetationsperiode 1999 und nach dem PENMAN-MONTEITH-Ansatz. Die Saftflusssumme für $P$. euphratica ist aus den lückenhaften Messwerten interpoliert und nur unter Vorbehalt zu betrachten (siehe Text).

\begin{tabular}{lcc}
\hline & Wasserverbrauch & \\
& & {$\left[\mathrm{kg} \mathrm{H}_{2} \mathrm{O}^{*} \mathrm{~m}^{-2}\right]$} \\
\hline & Berechnet mit & Berechnet mit \\
Pflanzenart & Saftflusssumme & Penman-Monteith-Ansatz \\
\hline Alhagi sparsifolia & 390,64 & 322,43 \\
Calligonum caput-medusae & 183,22 & 115,94 \\
Tamarix ramosissima & 440,54 & 127,83 \\
Populus euphratica & $(48,23)$ & 392,12 \\
\hline
\end{tabular}

\subsubsection{Produktion der untersuchten Arten}

Aufgrund der schlechteren Wasserversorgung ist die Produktion in Trockengebieten meist niedriger als in humiden Gebieten (LARCHER 1994). In dem hier beschriebenen Projekt war aber aufgrund des Zugangs der untersuchten Arten zum Grundwasser eine gute Wasserversorgung gewährleistet. Die Produktion der untersuchten Arten war hoch verglichen mit anderen Wüstengebieten (WHITTAKER \& NiERING 1975). SCHNEIDER (1993) fand in der südwest-ägyptischen Wüste bei Alhagi mannifera eine Jahresproduktion von 1,28 $\mathrm{t}^{*} \mathrm{ha}^{-1}$. ZHANG, GAO \& ZHENG (2002) berichten von einer Produktivität von $0,44 \mathrm{t}^{*} \mathrm{ha}^{-1}$ bis $1,1 \mathrm{t}^{*} \mathrm{ha}^{-1}$ von Pflanzengesellschaften am Nordrand der Taklamakan, die aus A. sparsifolia und Phragmites communis aufgebaut sind.

Allerdings wird für T. ramosissima bei LIU et al. (1988) eine Jahresproduktion von 4,19 $\mathrm{t}^{*} \mathrm{ha}^{-1}$ in einem dichten Bestand angegeben. WANG et al. (1996) haben in verschieden alten Mischbeständen mit $P$. euphratica, T. ramosissima und anderen Arten eine oberirdische Produktion von 0,21 - 0,90 t ha $^{-1}$ für T. ramosissima und $1,6-2,3 t^{*} a^{-1}$ für $P$. euphratica gemessen.

Im hier vorgestellten Projekt wurde auch in Anbetracht einer gute Wasserversorgung eine bemerkenswert hohe Produktion gemessen, wenn man den niedrigen Blattflächenindex (LAI) der Arten bedenkt. Die folgenden Angaben beziehen sich auf die unbewässerten ungenutzten Flächen: Bei Alhagi sparsifolia wurde 1999 eine oberirdische Produktion von 2,97 $\mathrm{t}^{*} \mathrm{ha}^{-1}$ ermittelt, bei Tamarix 
ramosissima 1,55 $\mathrm{t}^{*} \mathrm{ha}^{-1}$ (in geschlossenen Beständen 3,1 $\mathrm{t}^{\star} \mathrm{ha}^{-1}$ ). Populus euphratica erreichte $3,17 \mathrm{t}^{*} \mathrm{ha}^{-1}$ und bei Calligonum caput-medusae wurden sogar bis zu 11,25 $t^{*}$ ha $^{-1}$ gemessen (GRIES et al. eingereicht). Im Jahr 2000 war die Produktion bei $A$. sparsifolia und C. caput-medusae mit 1,59 $\mathrm{t}^{*}$ ha- ${ }^{-1}$ bzw. $8,55 \mathrm{t}^{*} \mathrm{ha}^{-1}$ geringer, bei $P$. euphratica mit $3,79 \mathrm{t}^{*} \mathrm{ha}^{-1}$ und $T$. ramosissima mit 2,24 $\mathrm{t}^{\star} \mathrm{ha}^{-1}$ noch höher als im Vorjahr. Damit reicht die oberirdische Produktion der Vorlandvegetation an die Produktionsleistungen von Wäldern gemäßigter Breiten heran (ELLENBERG et al. 1986).

\subsubsection{Wassernutzungseffizienz der Produktion (WUEp)}

Pflanzen in Trockengebieten sind häufig an Wasserknappheit angepasst, indem sie ihren Wasserverbrauch einschränken. Geringer Wasserumsatz bei relativ hoher Produktivität führt zu einer hohen Wassernutzungseffizienz der Produktion (WUEp). LARCHER (1994) gibt für $\mathrm{C}_{4}$-Pflanzen Werte von 3-5 $\mathrm{g} \mathrm{TG}^{*} \mathrm{~kg}^{-1} \mathrm{H}_{2} \mathrm{O}$ an, für krautige Leguminosen 1,3-1,4 $\mathrm{g} \mathrm{TG}^{*} \mathrm{~kg}^{-1} \mathrm{H}_{2} \mathrm{O}$ und für Hartlaubsträucher 3$6 \mathrm{~g} \mathrm{TG}^{*} \mathrm{~kg}^{-1} \mathrm{H}_{2} \mathrm{O}$. Dabei ist nicht klar, ob sich diese Werte auf die gesamte oder nur die oberirdische Produktion beziehen. CLIFTON-Brown \& LEWANDOWSKI (2000) berechneten für ver-schiedene Genotypen des $\mathrm{C}_{4}$-Grases Miscanthus die WUEp für die gesamte Pflanze mit 11,5-14,2 $\mathrm{g} \mathrm{TG}^{*} \mathrm{~kg}^{-1} \mathrm{H}_{2} \mathrm{O}$ und mit $1,9-4,4 \mathrm{~g} \mathrm{TG}^{*} \mathrm{~kg}^{-}$ ${ }^{1} \mathrm{H}_{2} \mathrm{O}$ nur für die oberirdische Biomasse.

Die Wassernutzungseffizienz der Produktion (WUEp) wurde als Quotient aus verbrauchten Wasser (Saftlusssumme) und oberirdischer Produktion (Holzzuwachs plus Blattmasse) berechnet. C. caput-medusae erreicht mit 1,16 g $\mathrm{TG}^{*} \mathrm{~kg}^{-1} \mathrm{H}_{2} \mathrm{O}$ den höchsten, A. sparsifolia mit $0,60 \mathrm{~g} \mathrm{TG}^{*} \mathrm{~kg}^{-1} \mathrm{H}_{2} \mathrm{O}$ den niedrigsten Wert (Abb. 4.7.1). Dies erscheint zunächst niedrig verglichen mit anderen Angaben. Für Tamarix ramosissima wurde von GLENN et al. (1998) eine WUEp von 1,8 $\mathrm{g} \mathrm{TG}^{*} \mathrm{I}^{-1} \mathrm{H}_{2} \mathrm{O}$ und für Populus fremontii $2,1 \mathrm{~g} \mathrm{TG}^{*} \mathrm{I}^{-1} \mathrm{H}_{2} \mathrm{O}$ angegeben, auch ANDERSON (1982) berechnete hohe Wassernutzungseffizienzen aus Photosynthesemessungen und Transpirationsraten von $4,3 \mathrm{mg} \mathrm{TG}^{*} \mathrm{~g}^{-1} \mathrm{H}_{2} \mathrm{O}$ (T. ramosissima) und 6,8 $\mathrm{mg} \mathrm{TG}^{*} \mathrm{~g}^{-1} \mathrm{H}_{2} \mathrm{O}$ (Populus fremontii).

Dagegen liegen die in vorliegender Arbeit berechneten Werte für $T$. ramosissima deutlich niedriger (Abb. 4.7.1). Die WUEp für Populus euphratica liegt zwar mit 
3,5 $\mathrm{g} \mathrm{TG}^{*} \mathrm{~kg}^{-1} \mathrm{H}_{2} \mathrm{O}$ in einer ähnlichen Größenordnung wie die für $P$. fremontii angegebenen Werte, dies liegt aber sicher an der unterschätzten Saftflusssumme (siehe Kapitel 5.4.2).

Mehrere Gründe könnten zu einer Unterschätzung der WUE beigetragen haben: nur die oberirdische Produktion konnte erfasst werden und es können Fehler bei der Hochrechnung der Produktion oder des Wasserverbrauches aufgetreten sein. Von Pflanzen in Trockengebieten wird beschrieben, dass die unterirdische Biomasse einen sehr großen Anteil an der Gesamtbiomasse hat (SCHENK \& JACKSON 2002). Dies trifft einerseits auf Pflanzen zu, die ein sehr weitreichendes Wurzelsystem ausbilden, um Niederschläge oder periodisch auftretendes Oberflächenwasser effizient auszunutzen (NOBEL \& LINTON 1997, GIBBENS \& LENZ 2001). Auch Pflanzen, in deren Wurzelraum in Trockenperioden plötzlich große Mengen an Feinwurzeln absterben, haben eine hohe unterirdische Produktivität. Bei Phreatophyten jedoch kann man davon ausgehen, dass sie vergleichsweise wenig Feinwurzeln produzieren müssen, da sie das Wasser aus gesättigten Bodenschichten beziehen. Die Länge der Wasserleitungswege ist zwar bei großer Entfernung zum Grundwasser lang (CANADELL et al. 1996), aber an das Grobwurzelsystem oder die unterirdischen Sprosse der vom Sand überwehten Pflanzen werden nur geringe Anforderungen bezüglich der mechanischen Festigkeit gestellt, denn das umgebende Bodenmaterial bietet eine stabile Stütze. So wurde z.B. das spontane Abbrechen eines aus einer Düne ausgegrabenen Stammes von Populus euphratica beobachtet, sobald der stützende Sand entfernt war (RUNGE, M., mündliche Mitteilung). In den meisten Untersuchungen zur WUEp, wurde ebenfalls nur die oberirdische Produktion berücksichtigt, daher sind Vergleiche möglich.

Bei den untersuchten Arten war der Basaldurchmesser bzw. die Basalfläche eng mit der Biomasse des Holzanteils korreliert, daher wurde die Produktivität mit allometrischen Regressionsgleichungen berechnet (GRIES et al. eingereicht). Die verwendeten Korrelationen zwischen der Basalfläche der Triebe bzw. dem Buschvolumen (im Falle von A. sparsifolia) und der Biomasse sind sehr eng (GRIES et al. eingereicht). Es wurde zudem eine große Anzahl von Büschen bzw. Trieben auf den Teilflächen vermessen und die Variabilität innerhalb der Bestände damit gut erfasst; daher ist von einer realistischen Erfassung der flächenbezogenen 
Produktion, zumindest nicht von einer groben Fehleinschätzung, auszugehen. Die Produktion wurde durch wiederholtes Messen zahlreicher markierter Triebe bestimmt und nicht direkt an den Pflanzen gemessen, an denen der Wasserhaushalt untersucht wurde. Der Wasserverbrauch (Saftfluss) wurde aber nur mit 6 Parallelen gemessen und für Teile des Jahres über die Strahlung modelliert und weist deswegen eine höhere Ungenauigkeit auf.

Aus den Leitfähigkeitsmessungen wurde mit dem PENMAN-MONTEITH-Ansatz (MONTEITH \& UNSWORTH 1990) ebenfalls der Wasserverbrauch während der Vegetationsperiode und damit die WUEp berechnet (THOMAS et al., in Vorbereitung). Der mit dieser Methode ermittelte Wasserverbrauch lag für Alhagi sparsifolia, Calligonum caput-medusae und Tamarix ramosissima niedriger als der aus dem Saftfluss errechnete Wert (Tab. 6). Mit dem PENMAN-MonTEITH-Ansatz ergibt sich für diese Arten eine höhere WUEp. Die Berechnungen nach PENMANMONTEITH beruhen auf nur vier bis fünf Messungen während der Vegetationsperiode und weisen damit einen höheren zufälligen Fehler auf. Bei den Saftflussmessungen kann ein größerer systematischer Fehler vorliegen, was vor allem bei $P$. euphratica zutrifft. Außerdem können die bei der Modellierung der Saftflusswerte aufgetretenen Schätzfehler einen Einfluss auf die Höhe der WUEp haben. Man kann daher von den beiden Methoden ausgehend eine Spanne der WUEp angeben (Tab. 7):

\section{Tab. 7: Wassernutzungseffizienz der Produktion (WUEp) 1999.}

Berechnet auf Basis der Saftflusssumme der Vegetationsperiode 1999 und nach dem PENMAN-MONTEITH-Ansatz (THOMAS et al., in Vorbereitung). In Klammern ist der Wert nur auf die genutzten Teile der Pflanze (= Holzproduktion, außer bei $A$. sparsifolia) bezogen angegeben.

\begin{tabular}{|c|c|c|}
\hline & Wassernutzungsef & (WUEp) \\
\hline & & $\left.\mathrm{g} \mathrm{H}_{2} \mathrm{O}^{-1}\right]$ \\
\hline Pflanzenart & $\begin{array}{l}\text { Berechnet mit } \\
\text { Saftflusssumme }\end{array}$ & $\begin{array}{l}\text { Berechnet mit } \\
\text { Penman-Monteith-Ansatz }\end{array}$ \\
\hline Alhagi sparsifolia & $\begin{array}{ll}0,60 & (0,60)\end{array}$ & $\begin{array}{ll}0,71 \quad(0,71) \\
\end{array}$ \\
\hline Calligonum caput-medusae & $1,16 \quad(0,50)$ & $(0,79)$ \\
\hline Tamarix ramosissima & $0,82 \quad(0,40)$ & $(0,57)$ \\
\hline Populus euphratica & -- & $(0,79)$ \\
\hline
\end{tabular}


An den niedrigen Werten der WUEp und an der hohen Produktion kann man ablesen, dass die untersuchten Arten offensichtlich nicht unter starkem Trockenstress leiden, sondern ausreichend wasserversorgt sind.

\subsubsection{Kohlenstoff-Isotopen-Verhältnis $\left(\delta^{13} \mathrm{C}\right)$}

Das Kohlenstoff-Isotopenverhältnis $\left(\delta^{13} \mathrm{C}\right)$ ist ein integrierendes Maß für die WUE der Photosynthese (TOFT, ANDERSON \& NOWAK 1989, DEVITT et al 1997). Die stomatäre Leitfähigkeit beeinflusst direkt Transpiration $(E)$ und Photosynthese $(A)$ : die Transpirationsraten steigen linear und die Photosyntheseraten asymptotisch mit ansteigender Leitfähigkeit. Daher ist die photosynthetische WUE (A/E) mit der stomatären Leitfähigkeit negativ korreliert (FARQUHAR \& RICHARDS 1984, EHDAIE et al. 1991).

Bei Pflanzen in Gebieten mit geringeren Niederschlägen wurden häufig höhere $\delta^{13} \mathrm{C}$-Werte gefunden als bei Pflanzen in besser wasserversorgten Gebieten (NEWTON, DICK \& HEATON 1996), allerdings fanden SCHULZE et al. (1991) keinen eindeutigen Bezug zwischen Aridität und $\delta^{13} \mathrm{C}$-Wert.

EHLERINGER, PHILLIPS \& COMSTOCK (1992) beobachteten bei verschiedenen Wüstenpflanzen eine saisonale Veränderung der Isotopendiskriminierung. Mit zunehmendem Trockenstress stieg der $\delta^{13} \mathrm{C}$-Wert an. Ein Anstieg der $\delta^{13} \mathrm{C}$-Werte bei zunehmendem Wasserdefizit wurde auch von ARNDT et al. (2001) bei Ziziphus rotundifolia gefunden. Da in vorliegender Arbeit zunächst die Hypothese aufgestellt worden war, dass die Pflanzen zunehmendem Trockenstress ausgesetzt sein würden, war auch ein solcher Effekt bei der Isotopensignatur erwartet worden.

DEVITT et al. (1997a) fanden, dass Kohlenstoffisotopenverhältnisse bei Bewässerungsexperimenten mit getopften Pflanzen von Quercus virginiana und Festuca arundinacea gute Indikatoren für die WUEp der Blätter darstellen. Allerdings müssen die Pflanzenart, Größe und Wachstumsbedingungen einbezogen werden, da es interspezifische und intraspezifische Unterschiede (z.B. je nach Alter) gibt. In einer früheren Studie aber (DEVITT, MORRIS \& NEUMANN 1994) wurde keine Korrelation zwischen $\delta^{13} \mathrm{C}$ und der WUEp beobachtet. Da man die WUE mit dem gesamten oberirdischen Trockengewicht berechnet hatte, nicht nur mit dem 
Blatttrockengewicht, wirkte sich der applizierte Trockenstress erst nach der Bildung eines Teils der Holzbiomasse aus.

Für Pflanzenarten in extrem trockenen Gebieten geben STEWART et al. (1995) $\delta^{13} \mathrm{C}$-Werte von $-20,00$ bis $-26,00 \%$ an. Alhagi sparsifolia und Populus euphratica zeigen im Sommer deutlich negativere Werte, was auf eine gute Wasserverfügbarkeit hinweist. Die $\mathrm{C}_{4}$-Pflanze $C$. caput-medusae zeigt wie erwartet die am wenigsten negativen Kohlenstoff-Isotopen-Verhältnisse der untersuchten Arten, da das $\mathrm{CO}_{2}$ über die nicht gegen ${ }^{13} \mathrm{CO}_{2}$ diskriminierende PEP-Carboxylase vorfixiert wird. Im Artvergleich hat $C$. caput-medusae aufgrund des $\mathrm{C}_{4^{-}}$ Metabolismus also die höchste Wassernutzungseffizienz und die höchsten $\delta^{13} \mathrm{C}$ Werte. Die Gattung Calligonum weist den NAD-ME Typ (bei der $\mathrm{CO}_{2}$-Fixierung über Aspartat und Malat sind die Mitochondrien der Bündelscheidenzellen beteiligt, siehe SAGE \& MONSON 1999) des $C_{4}$-Metabolismus auf (PYANKOV et al. 2000), für den ein durchschnittlicher $\delta^{13} \mathrm{C}$-Wert von $-11,4 \%$ bis $-12,7 \%$ angegeben wird (CERLING 1999).

Zum Vergleich zwischen Isotopensignatur und Wassernutzungseffizienz sollte man den $\delta^{13} \mathrm{C}$-Wert der Blatternte zur Zeit der maximalen Biomasseentwicklung im August heranziehen, da dieser einen integrierten Wert über die Wachstumsperiode widerspiegeln und noch nicht von Seneszenzerscheinungen beeinflusst sein sollte. Bei den $\mathrm{C}_{3}$-Arten wurden die höchsten $\delta^{13} \mathrm{C}$-Werte bei $T$. ramosissima gemessen, deutlich niedrigere bei $A$. sparsifolia und $P$. euphratica. $T$. ramosissima erreichte auch eine höhere WUEp als $A$. sparsifolia, was mit den höheren $\delta^{13} \mathrm{C}$-Werten zusammenpasst. Ein Vergleich zwischen WUEp und $\delta^{13} \mathrm{C}$ für $P$. euphratica und $A$. sparsifolia ist schwierig, da das Verhältnis von transpirierender Biomasse zu bestehender oberirdischer Biomasse bei den beiden Arten zu unterschiedlich ist. Man sieht, dass ein Artvergleich der WUE über das Isotopenverhältnis problematisch ist (siehe auch DEVITT et al. 1997). Außerdem sollte man berücksichtigen, dass bei ausreichender Wasserzufuhr das Verhältnis zwischen WUE und $\delta^{13} \mathrm{C}$-Wert nicht mehr so eng ist wie bei trockengestressten Pflanzen.

Bei allen untersuchten Arten wurde jedoch ein Absinken des Isotopenverhältnisses im Jahresverlauf beobachtet (Abb. 4.8.1). Die positiveren $\delta^{13} \mathrm{C}$-Werte im Frühjahr können auf unterschiedliche Ursachen zurückzuführen sein. Zum einen beeinflussen klimatische Parameter die Isotopendiskriminierung. Bis auf die 
Lufttemperatur unterscheiden sich diese nicht von April zu den anderen Messterminen. Das VPD war schon im April 1999 sehr hoch, die relative Luftfeuchte sehr niedrig. SMITH et al. (1976) zeigten, dass bei niedrigeren Temperaturen die Diskriminierung gegen ${ }^{13} \mathrm{C}$ geringer und damit der $\delta^{13} \mathrm{C}$-Wert positiver ist: eine um etwa $5{ }^{\circ} \mathrm{C}$ höhere Temperatur kann sich in einem um 1-2 $\%$ niedrigeren $\delta^{13} \mathrm{C}$-Wert bemerkbar machen. Auch MORECROFT \& WOODWARD (1990) sowie EDWARDS et al. (2000) beobachteten diesen Temperatureinfluss auf die Isotopensignatur. Im April lagen die mittleren Lufttemperaturen mit $15,35^{\circ} \mathrm{C}$ (Qira_1) bzw. $17.90^{\circ} \mathrm{C}$ (Qira_2) deutlich niedriger als in den Sommermonaten (Tab. 8).

Tab. 8: Monatsmittel der Lufttemperaturen der Jahre 1999 und 2000.

Werte der Wetterstationen auf der Alhagi-Fläche (Qira_1) und der Tamarix-Fläche (Qira_2).

\begin{tabular}{l|cc|cc|c}
\hline \multicolumn{7}{c}{ Mittlere Lufttemperaturen [ $\left.{ }^{\circ} \mathbf{C}\right]$} \\
Monat & Qira_1 & Qira_2 & Qira_1 & Qira_2 & Qira_2 \\
& 1999 & 1999 & 2000 & 2000 & 2001 \\
\hline Januar & - & - & $-3,64$ & - & $-5,27$ \\
Februar & - & - & $-2,08$ & - & 0,62 \\
März & - & - & 7,14 & - & 9,33 \\
April & 15,35 & 17,90 & 17,59 & - & 16,72 \\
Mai & 22,34 & 22,16 & 23,62 & 23,36 & - \\
Juni & 25,50 & 25,09 & 24,76 & 24,67 & - \\
Juli & 25,76 & 25,69 & 26,30 & 26,28 & - \\
August & - & 24,82 & 25,24 & 25,40 & - \\
September & - & 22,33 & - & 19,41 & - \\
Oktober & 11,41 & - & - & 12,03 & - \\
November & 2,96 & - & - & 3,01 & - \\
Dezember & $-2,83$ & - & - & $-4,32$ & -
\end{tabular}

Ein wichtiger Grund für die positiveren $\delta^{13} \mathrm{C}$-Werte der Blätter im April und Mai kann die Folge einer internen Kohlenstoffdiskriminierung sein. Die frisch austreibenden Blätter laubwerfender Arten werden im Frühjahr zunächst mit Reservekohlenhydraten versorgt (MAILLARD et al. 1994). Sekundäre Produkte wie Stärke sind aufgrund metabolischer Prozesse mit ${ }^{13} \mathrm{C}$ angereichert. Stärke zeigt bei verschiedenen Pflanzenarten im Vergleich zu Saccharose einen deutlich positiveren $\delta^{13} \mathrm{C}$-Wert (GLEIXNER et al. 1998, WANEK et al. 2001). Wenn also ein höherer Anteil an Reservekohlenstoff in die Blätter eingebaut wird, führt dies dort zu einem Anstieg der $\delta^{13} \mathrm{C}$-Werte. In einem Regenwald in Panama wurde bei 
verschiedenen Arten beobachtet, dass frisch ausgetriebene Blätter ein positiveres Kohlenstoffisotopen-Verhältnis hatten als ältere Blätter des gleichen Baumes (TERWILLIGER et al. 2001). Es wurde daraus geschlossen, dass die Anreicherung mit ${ }^{13} \mathrm{C}$ durch die Translokation von Kohlenstoff in die neuen Blätter bedingt war. Der signifikant positivere $\delta^{13} \mathrm{C}$-Wert auf der ADN- und AFU-Fläche Ende August 2000 (Abb. 4.8.2) könnte vielleicht auf eine Probenahme jüngerer Blätter als an den anderen Terminen zurückzuführen sein. Die Blätter wurden von einem anderen Bearbeiter geerntet. Ein Absinken der $\delta^{13} \mathrm{C}$-Werte mit dem Blattalter (im Verlauf der Vegetationsperiode) wurde auch von DAMESIN et al. (1997) an Quercus pubescens und Quercus ilex gemessen.

Vergleicht man den Verlauf der $\delta^{13} \mathrm{C}$-Werte mit dem Verlauf der Blattmassenentwicklung, kann man feststellen, dass die Kurven genau entgegengesetzt verlaufen (Abb. 4.3.1 und 4.8.1). Dies stützt die Erklärung, dass die andere Isotopensignatur der Speicherstoffe für den Jahresverlauf verantwortlich ist. Wenn die Blattbiomasse voll entwickelt ist und nicht mehr auf Speicherstoffe angewiesen, hat auch der $\delta^{13} \mathrm{C}$-Wert seinen tiefsten Punkt erreicht.

Auch eine Änderung der Sensitivität der Stomata im Jahresverlauf könnte Auswirkungen auf das Kohlenstoffisotopenverhältnis haben. NILSEN et al. (1983) konnten einen Zusammenhang zwischen Blattleitfähigkeit und Blattalter feststellen. So kann man annehmen, dass jüngere Blätter auf einen Anstieg des VPD mit einer stärkeren Verringerung der stomatären Leitfähigkeit reagieren als ältere Blätter. Dadurch kann sich durch den reduzierten Wasserverbrauch eine höhere WUE und damit ein positiverer $\delta^{13} \mathrm{C}$-Wert ergeben. Gegen diese Erklärung für die positiveren $\delta^{13} \mathrm{C}$-Werte im Frühjahr sprechen die sehr hohe stomatäre Leitfähigkeit und hohen Transpirationsraten während der ersten Messkampagne 1999 (Abb. 4.5.2 und 4.5.10).

Die $\delta^{13} \mathrm{C}$-Werte zeigten bei allen untersuchten Arten einen ähnlichen Jahresverlauf, der nicht durch die Bewässerung beeinflusst wurde, sondern durch metabolische Prozesse verursacht wird. Ein Vergleich mit der Wassernutzungseffizienz der Produktion ist nur bedingt möglich, da bei ausreichender Wasserverfügbarkeit keine enge Beziehung zu erwarten war. 


\subsection{Nutzung der untersuchten Arten}

Nachdem im Spätsommer 1999 die experimentelle Nutzung auf den Untersuchungsflächen erfolgt war, wurden Wasserpotentiale, Transpiration und stomatäre Leitfähigkeit in der Vegetationsperiode 2000 bei C. caput-medusae, $T$. ramosissima und $P$. euphratica parallel auf den im Vorjahr genutzten und den ungenutzten Flächen gemessen.

Da sich alle untersuchten Arten durch vegetative Vermehrung / klonales Wachstum auszeichnen (z.B. KERR et al. 1965, SHARMA et al. 1999, QONG et al. 2002), wurde davon ausgegangen, dass bei der Auflichtung nicht konkurrierende Nachbarindividuen, sondern konkurrierende Triebe der gleichen Pflanze entfernt worden waren. Zur Auswirkung der Auflichtung durch die experimentelle Nutzung auf Wasserhaushalt und Produktivität gab es zwei gegensätzliche Hypothesen:

1. Wenn bei gleichbleibender unterirdischer hydraulischer Leitfähigkeit weniger Triebe versorgt werden müssen, treten weniger negative Wasserpotentiale auf, was eine höhere stomatäre Leitfähigkeit und Transpiration ermöglicht. Dadurch steigt die Produktion auf der genutzten Fläche.

Bei konkurrierenden Individuen sind solche positiven Effekte einer Auflichtung auf den Wasserhaushalt und die Produktivität aus verschiedenen Untersuchungen bekannt (Bréda, Granier \& Aussenac 1995, Smit \& Rethman 2000). Wenn die Konkurrenz um Wasser und Nährstoffe im Boden abnimmt und weniger gegenseitige Beschattung auftritt, steigt die Produktivität an (SINGH \& THOMPSON 1995, LÓPEZ VILLAGRA \& FELKER 1997).

2. Weil durch weniger Assimilationsorgane mehr unterirdische Biomasse unterhalten werden muss, sinkt unabhängig von der Wasserversorgung die Produktion auf der genutzten Fläche.

Durch die Auflichtung der Bestände können gleichzeitig sowohl die Strahlungsintensität als auch das VPD im Kronenraum zunehmen. Die beiden Faktoren wirken gegensätzlich auf die stomatäre Leitfähigkeit, aber beide fördernd auf die Transpiration; eine Vorhersage der Effekte ist daher schwierig. Bei den ohnehin sehr lockeren Kronen der untersuchten Arten und der hohen Strahlungsintensität im Untersuchungsgebiet dürfte weder eine gegenseitige Beschattung noch ein 
Gradient des VPD zwischen innerer Krone und Umgebung auftreten. Mit bedeutenden Auflichtungseffekten auf das Klima im Kronenraum ist daher nicht zu rechnen.

\subsubsection{Auswirkung der experimentellen Nutzung auf den Wasserhaushalt}

Nur bei $P$. euphratica wurde ein signifikanter Unterschied im Mittagsblattwasserpotential zwischen ungenutzter und genutzter Teilfläche festgestellt. Die Wasserpotentiale waren auf der genutzten Fläche höher, was darauf hindeutet, dass die Bäume dort nach der Auflichtung besser wasserversorgt waren. Bei den anderen Arten zeigten sich weder bei den predawn- noch den Mittagswasserpotentialen signifikante Unterschiede zwischen den Flächen.

Bei $P$. euphratica wurde nur im August eine höhere Transpiration auf der aufgelichteten Fläche gemessen, in den anderen Monaten gab es keine signifikanten Unterschiede. Das VPD auf der genutzten Fläche war bei den Messungen im August und September zwar höher, im Juni dagegen geringer als auf der ungenutzten Fläche. Schon 1999 wurde festgestellt, dass die Transpiration bei $P$. euphratica kaum mit dem VPD korreliert.

Bei C. caput-medusae sind nur im Mai VPD und Wasserverbrauch auf der genutzten Fläche höher, im weiteren Jahresverlauf sind die Flächen gleich oder die ungenutzte weist höhere Werte auf. Dass kein deutlicher Effekt der Auflichtung auf den Wasserhaushalt bei C. caput-medusae gefunden wurde, lässt sich zum Teil durch die nur auf den Probequadraten und nicht auf der gesamten Fläche durchgeführte Nutzung erklären. Dadurch wurde vermutlich nicht für alle Pflanzen eine reale Auflichtung erreicht.

Bei $T$. ramosissima konnte dagegen an beiden Terminen unabhängig vom VPD im Bestand auf der genutzten Fläche ein signifikant höherer Wasserverbrauch festgestellt werden. Also nimmt bei T. ramosissima offensichtlich die Konkurrenz um Wasser ab. Da man nicht bestimmen kann, welche Triebe bei T. ramosissima als ein Individuum anzusehen sind, ist nicht festzustellen, welcher Effekt zum Tragen kam: musste ein Individuum nach der Nutzung eine verringerte Triebzahl 
mit der gleichen verfügbaren Wassermenge versorgen oder wurde ein konkurrierender Nachbarbusch entfernt, so dass mehr Wasser für das verbleibende Individuum zur Verfügung steht?

Nur bei $A$. sparsifolia war keine Veränderung der Nutzung auf den Wasserhaushalt erwartet worden, da nicht aufgelichtet, sondern die Fläche komplett abgeerntet wurde. Es wurde davon ausgegangen, dass die Produktivität der beernteten Fläche im Folgejahr abnimmt (KERR 1965, GRIES et al. eingereicht), da durch den schon im Spätsommer stattfindenden Biomasseentzug weniger Reservestoffe gebildet werden können. Auch bei $A$. sparsifolia wurde allerdings ein Anhaltspunkt für Konkurrenz zwischen den Büschen gefunden. Die Größe der Einzelbüsche nahm signifikant $z u$, wenn die Bestandesdichte abnahm (GRIES, D. unveröffentlicht), was bei diesem ohnehin recht lockeren Bestand nicht mit höherem Lichtgenuss erklärt werden kann, sondern nur mit einer besseren Wasserversorgung der verbleibenden Büsche.

\subsubsection{Auswirkungen der experimentellen Nutzung auf die Produktion}

Die Produktivität der verbleibenden Triebe bzw. Bäume nahm im Untersuchungsjahr 2000 bei $T$. ramosissima und $P$. euphratica zu, während bei $C$. caput-medusae auf der CF-Fläche eine vergleichbare Produktivität wie 1999 gemessen wurde (GRIES et al. eingereicht). Auf den anderen Teiflächen von $C$. caput-medusae wurde für 2000 eine geringere Produktion als 1999 berechnet. Wenn man also von einem weniger produktiven Jahr 2000 ausgeht, kann man auch bei $C$. caput-medusae einen positiven Effekt der Auflichtung auf den Zuwachs der verbleibenden Triebe ausmachen. Bei $P$. euphratica liegen nur im August 2000 die Transpiration und Leitfähigkeit auf der genutzten Fläche über den Werten der ungenutzten Fläche. 1999 war das Wachstum der Pappeln aber im Juli schon abgeschlossen (GRIES et al. eingereicht) und für 2000 liegen keine saisonalen Zuwachsdaten vor. Es ist fraglich, ob die Bäume der PFU-Fläche im Jahr 2000 bis in den August hinein Zuwachs aufwiesen und so von einer höheren Leitfähigkeit im Sommer profitieren konnten. Die Bäume auf der genutzten Fläche 
entwickelten allerdings 2000 bei gleichem Durchmesser eine höhere Blattmasse als die Bäume auf der ungenutzten Fläche (Gries, D., unveröffentlicht). Dies kann zu dem größeren Zuwachs auf der PFU-Fläche beigetragen haben.

A. sparsifolia zeigte auf den genutzten Flächen verglichen mit den Werten von 1999 eine geringere Produktivität im Jahr 2000. Kein Effekt wurde aber durch die alternative Art der Ernte (reduced use, Flächen AFR und ADR) gefunden. Es war vermutet worden, dass durch ein Abschneiden der Büsche an der Bodenoberfläche die unterirdischen Erneuerungsknospen geschont werden (GRIES et al. eingereicht).

Die Nutzung hatte also wie erwartet einen negativen Effekt auf die Produktivität von A. sparsifolia (GRIES et al. eingereicht). Dagegen konnte man feststellen, dass eine mäßige Nutzung der anderen untersuchten Arten durch die höhere Produktion der verbleibenden Triebe bzw. Stämme teilweise ausgeglichen wurde.

\subsubsection{Nutzung durch die Bevölkerung}

Von den untersuchten Pflanzenarten unterliegt laut den Ergebnissen einer Umfrage in 30 bäuerlichen Haushalten (ZHENG et al. 2001) C. caput-medusae dem geringsten Nutzungsdruck. Tamarix stellt $80 \%$ des benötigten Brennholzes und ebenfalls $80 \%$ des Baumaterials, das dem Oasenvorland entnommen wird, während Populus euphratica $15 \%$ des Brenn- und $10 \%$ des Baumaterials liefert. Außerdem trägt $P$. euphratica zu $20 \%$ der Futtermenge bei. Hier wurden vermutlich auch Populus alba und $P$. nigra mitgerechnet, die innerhalb der Oase als Windschutz und Bauholz gepflanzt werden. A. sparsifolia stellt $45 \%$ der benötigten Futtermenge und $20 \%$ des Gründüngers.

Populus euphratica wird nach PAN \& YING (1995) nicht nur zur Werkzeug- und Möbelherstellung sowie als Viehfutter genutzt, sondern es wird auch das Salz des bei Verletzungen austretenden Xylemsaftes gewonnen. Neben den im vorgestellten Projekt untersuchten Pflanzenarten werden auch weitere Arten des Oasenvorlandes als Futterpflanzen (z.B. Karelinia caspica, Phragmites australis), als Gründünger (Sophora alopecuroides) oder für medizinische Zwecke (z.B. Cistanche spec.) genutzt (ZHENG et al. 2001, LIU, J. unveröffentlicht). 
Durch die rasch wachsende Bevölkerung in den Oasen steigt der Nutzungsdruck auf die Vorlandvegetation weiterhin an, daher ist ein Managementprogramm zur nachhaltigen Nutzung unabdingbar. Jede Familie benötigt etwa $960 \mathrm{~kg}$ Feuerholz im Jahr (ZHENG et al. 2001). In einem Teil des Jahres wird auch Kohle verwendet. $90 \mathrm{~kg}$ Tamarixtriebe pro Haushalt und Jahr sind als Baumaterial erforderlich. Die im Projekt ermittelte Produktivität von T. ramosissima erreichte 1999 (vor der Nutzung) 0,19 - 1,34 $t^{*} \mathrm{ha}^{-1}$ auf den ungenutzten und 1,76 - 3,3 $\mathrm{t}^{*} \mathrm{ha}^{-1}$ auf den genutzten Teilflächen. Die Basalfläche der Triebe nahm im Folgejahr um $25 \pm 6 \%$ auf den ungenutzten und um $61 \pm 8 \%$ auf den genutzten Flächen zu (GRIES et al. eingereicht). Durch die erhöhte Produktivität der verbliebenen Triebe nach der Nutzung konnte ein Teil der entnommenen Biomasse kompensiert werden. Für die Produktivität der gesamten Vorlandfläche müssen aber geringere Werte angenommen werden, da $T$. ramosissima nicht überall so dichte Bestände ausbildet, wie auf den in vorliegender Arbeit untersuchten Flächen. Wenn man mit einer mittleren Produktivität der Tamarix-Arten von $0,5 \mathrm{t}^{*}$ ha ${ }^{-1}$ für das Vorland rechnet, so müsste jede Familie etwa zwei Hektar Tamarix pro Jahr beernten können, damit eine nachhaltige Nutzung gewährleistet ist. In dem im hier vorgestellten Projekt kartierten Gebiet von $170 \mathrm{~km}^{2}$ im westlichen Oasenvorland (Abb. 2.1.2) gab es knapp $21 \mathrm{~km}^{2}$ mit von Tamarix bewachsenen Dünen und etwa $28 \mathrm{~km}^{2}$ einer Mischvegetation aus Tamarix, Alhagi sparsifolia und Karelinia caspica. Ohne genauere Zahlen zur Produktivität und zum Verbrauch kann man nicht voraussagen, wie stark die Tamarisken im Vorland übernutzt werden. Es sind jedoch überall Anzeichen einer Degradation der Bestände zu erkennen, da häufig auch alte Wurzelstöcke und dickere Triebe ausgegraben und als Brennholz genutzt werden. Es scheint aber, dass mit einem Managementplan und Regenerationsmaßnahmen eine nachhaltige Nutzung von Tamarix möglich ist.

Pro Schaf wird ein Grünfutterverbrauch von $490 \mathrm{~kg}$ Trockengewicht pro Jahr angegeben, so dass sich ein Verbrauch von $53000 \mathrm{t}$ für den Viehbestand eines Dorfes mit 6000 Einwohnern ergibt (ZHENG et al. 2001). Nach oben genannten Angaben werden also von einer Dorfgemeinschaft jährlich etwa $24000 \mathrm{t} A$. sparsifolia als Futter benötigt. Auf den im hier vorgestellten Projekt untersuchten Flächen hatte Alhagi sparsifolia eine Produktion von 1,4 $\pm 0,34$ bis 3,85 $\pm 0,33$ t*ha $^{-1}$ (1999) und $0,42 \pm 0,12$ bis $1,7 \pm 0,23 t^{*} h^{-1}$ im Jahr 2000 (GRIES et al. eingereicht). In den meisten Bereichen des Vorlandes steht $A$. sparsifolia jedoch viel weniger dicht 
(BRUELHEIDE, H. unveröffentlicht). Daher ist eine Schätzung der gesamten Produktion von Alhagi sparsifolia im Vorland mit den vorliegenden Daten nicht möglich. Die von ZHENG et al. (2001) erhobenen Werte zur Nutzung der Pflanzen im Oasenvorland bieten aufgrund der kleinen Datengrundlage natürlich nur eine sehr grobe Schätzung der geernteten Mengen und überschätzen mit Sicherheit die tatsächlichen Mengen. Selbst wenn man die Produktivität von A. sparsifolia mit 1 $\mathrm{t}^{\star}$ ha ${ }^{-1}$ schätzen würde, was sehr hoch gegriffen ist, würde nur eine der sieben Dorfgemeinschaft der Oase Qira 24000 ha gleichmäßig dichten Alhagi-Bestand im Jahr abernten. Dies ist vermutlich mehr $A$. sparsifolia als im gesamten Vorland vorhanden ist. Im untersuchten Teil des Oasenvorlandes (Abb. 2.1.2) finden sich dichte Alhagi-Bestände nur in einem schmalen Bereich um die Oase, außerdem gibt es etwa $28 \mathrm{~km}^{2}$ einer Mischvegetation mit Tamarix spec., Alhagi sparsifolia und Karelinia caspica (BRUELHEIDE, H., unveröffentlicht), die jedoch eine deutlich geringere Produktion bezogen auf $A$. sparsifolia aufweisen muss. An dieser kurzen Hochrechnung sieht man, dass die Werte von ZHENG et al. (2001) für die geernteten Mengen an A. sparsifolia zu hoch liegen müssen.

Die Ergebnisse der Befragung stellen aber die Bedeutung der Vorlandvegetation für die bäuerliche Bevölkerung heraus. Die zunehmende Übernutzung wird unter ande-rem dadurch deutlich, dass die Bauern immer weitere Wege zurücklegen müssen, um eine Ladung Futter oder Brennholz zu finden (COQUE et al. 1991, ZHENG et al. 2001).

ZHENG et al. (2001) geben an, dass der Verbrauch von Baumaterial aus der Vorlandvegetation mit der zunehmenden Anpflanzung von Populus alba und $P$. nigra in der Oase zurückgeht, ebenso mit der Zunahme von chemischem Dünger die Verwendung von Gründünger. Bei Erkundigungen vor Ort wurde aber festgestellt, dass für viele Bauern in den ärmeren Dörfern die Verwendung von gekauftem Bauholz und Kunstdünger zu teuer ist (ZHANG, X., mündliche Mitteilung). Es ist daher fraglich, ob der Nutzungsdruck auf die Vorlandvegetation in den kommenden Jahren abnehmen kann. 


\subsubsection{Bedeutung der Wassernutzungseffizienz der genutzten Arten}

Calligonum caput-medusae weist bezogen auf die gesamte oberirdische Biomasse die höchste Wassernutzungseffizienz der untersuchten Arten auf. Da aber $C$. caput-medusae nur mit $5 \%$ zur benötigten Futtermenge beiträgt und es bei der vorliegenden Befragung überhaupt nicht als Brennholzquelle erwähnt wird (ZHENG et al. 2001), tritt zur Zeit seine Bedeutung als nutzbare Pflanze für die örtliche Bevölkerung in Qira in den Hintergrund. Dies könnte aber auch darauf zurückzuführen sein, dass C. caput-medusae im Untersuchungsgebiet nicht heimisch ist und daher nicht so stark in die Nutzung einbezogen wird. Als geeignete Art für Regenerationsmaßnahmen kann Calligonum caput-medusae aber wertvoll sein. Betrachtet man nur die WUEp der genutzten Anteile der Pflanzen, zeigen die Arten ähnliche Werte: $A$. sparsifolia hat dann eine mindestens ebenso hohe WUEp wie die anderen Arten, da die oberirdischen Teile der Pflanze komplett genutzt werden (Abb. 4.7.1). Eine Förderung von $A$. sparsifolia und $T$. ramosissima erscheint unter dem Aspekt der Nutzbarkeit vordringlich. Im Falle von C. caput-medusae wäre zu überprüfen, inwiefern die Art zur Futterversorgung beitragen und damit außer als Dünenfixierung und Brennholzlieferant auch für die Tierhaltung genutzt werden kann. $P$. euphratica als geschützte Art sollte ebenfalls gefördert werden, bis sich die Bestände erholt haben. Prinzipiell sind PopulusBestände eine bedeutende Ressource für Brenn- und Bauholz und könnten zudem als innerer Schutzgürtel um die Oasen einen Beitrag zur Verhinderung von Sandverwehung leisten. Es ist aber unverzichtbar auch die Regeneration der anderen Arten des Oasenvorlandes voranzutreiben, da auch sie eine wichtige Rolle bei der Fixierung von Dünen und als Schutz vor Sanddrift spielen (DREGNE 1988, MAKI et al. 1993) oder ebenfalls von der Bevölkerung genutzt werden.

\subsection{Regeneration}

In historischer Zeit befanden sich die Oasen am Südrand der Taklamakan viele Kilometer weiter im Norden, dort wo auch die Südroute der Seidenstraße verlief (YANG 1991, TIAN \& SONG 1997). Diese Oasen mussten aufgegeben werden, weil sich die Wassermenge der Flüsse aus dem Kunlun, bedingt durch den Rückgang 
der Gletscher, verringerte (HOYANAGI 1965), und sind heute oft von Sand überdeckt. Noch vor etwa 100 Jahren durchfloss der Keriya die Taklamakan und mündete in den Tarim (HEDIN 1899). Auch der Hotan-Fluss, der heute nur noch während der Sommerflut den Tarim erreicht (CHENG 1997), durchfloss ganzjährig die Wüste. Der ständige Rückgang der Wassermenge zusammen mit der wachsenden Bevölkerung, dem zunehmenden Anbau von bewässerungsintensiven Kulturen wie Baumwolle (HALIK, Ü. unveröffentlicht) sowie der Ausweitung der landwirtschaftlichen Nutzfläche, hat zu vermehrter Bohrung von Tiefwasserbrunnen geführt. In Qira sind zwischen 1997 und 2001 etwa 66 zusätzliche Brunnen entstanden (LU, Wasserbauamt Qira, mündliche Mitteilung), die vor allem Wasser aus 70 bis $90 \mathrm{~m}$ Tiefe pumpen. Einige der älteren Brunnen erschließen überdies den höher gelegenen Grundwasserhorizont, der auch die Wasserquelle für die Vorlandvegetation bildet. Es ist bisher wenig darüber bekannt, wie sich der steigende Wasserverbrauch in der Oase auf den Grundwasserstand im Oasenvorland aufwirkt und ob sich die in der Oase beobachteten Schwankungen bis ins Vorland erstrecken. Obwohl die untersuchten Arten große Entfernung zum Grundwasser ertragen und durch Sandakkumulation mit den Dünen emporwachsen können, wurde noch nicht untersucht, wie empfindlich sie auf rasche Absenkung des Grundwassers reagieren. Das großflächige Absterben der Tugaiwälder (von $P$. euphratica dominierte Auenwälder) am Tarim (OVEZLIEV et al. 1993, TIAN \& SONG 1997) oder am Amudarya (hier mit Populus ariana; TRESHKIN 2001) wird allgemein auf geringeren Abfluss und absinkendes Grundwasser zurückgeführt. Detaillierte Untersuchungen, die auch den Wasserhaushalt und die Produktivität der Pflanzen berücksichtigen, gibt es jedoch nicht.

Da die Entfernung zum Grundwasser an den meisten Stellen in Oasenvorland sehr groß und die Niederschlagsmenge zu gering ist, wird für eine Etablierung von Pflanzen auf zur Zeit vegetationsfreien Flächen in jedem Falle künstliche Bewässerung benötigt. Diese Initialbewässerung muss ausreichend sein, damit die Pflanzen das Grundwasser erreichen. Es muss also für verschiedene Pflanzenarten ein aufgrund der Geschwindigkeit ihres Wurzelwachstums maximaler Grundwasserabstand und die erforderliche Bewässerungsmenge ermittelt werden. Zudem muss vor Regenerationsmaßnahmen die Salinität des Bodens und des Grundwassers überprüft werden, um die geeigneten 
Pflanzenarten auszuwählen. Von den in der vorliegenden Arbeit untersuchten Arten ist die salztoleranteste Tamarix ramosissima, die laut ZHAO et al. (1997) $50 \mathrm{~g}$ Salz pro Liter Wasser verträgt und auch in anderen Arbeiten als sehr salztolerant bezeichnet wird (KLEINKOPF \& WALLACE 1974, DI TOMASO 1998, GLENN et al. 1998). Auch Alhagi sparsifolia (Alhagi pseudalhagi) wird als Halophyt geführt (KASSAS 1952, KURBAN et al. 1998, JIN 2002, ZHANG, GAO \& ZHENG 2002), wogegen Calligonum caput-medusae mäßig salztolerant ist. Populus euphratica ist nur in der Etablierungsphase etwas empfindlicher gegen Salz, als adulter Baum jedoch sehr salztolerant (MA et al. 1996, ZHAO et al. 1997) und wird als die geeignetste Baumart in salinisierten Trockengebieten bezeichnet (SHARMA et al. 1999).

Erfahrungen mit Regenerationsmaßnahmen mit Stecklingen verschiedener Pflanzenarten gibt es in allen zentralasiatischen Trockengebieten. Aus Versuchen in Turkmenistan ist bekannt, dass Calligonum caput-medusae Stecklinge bei einer Bewässerung, die eine Durchfeuchtung des Bodens bis in $1 \mathrm{~m}$ oder $2 \mathrm{~m}$ Tiefe gewährleistet, eine Überlebensrate von 52-64 \% bzw. 65-80 \% aufweisen (YARASHOV 1996). Auch LALYMENKO \& LALYMENKO (1997) haben Topfungsexperimente u.a. mit Calligonum-Arten und Tamarix ramosissima durchgeführt, um die optimalen Bedingungen zur Etablierung zu finden. In einem groß angelegten Bewässerungsprojekt am Südrand der Taklamakan wurde auf grundwassernahen Flächen im Vorland verschiedener Oasen erfolgreich Tamarix etabliert (MIDDLETON \& ThOMAS 1992). Eine einmalige Bewässerung mit $750-1500 \mathrm{~m}^{3 *} \mathrm{ha}^{-1}$ reichte aus, dass die Pflanzen überlebten.

Nach der einmaligen Überflutung wurden auf den Untersuchungsflächen im Falle von $A$. sparsifolia und $P$. euphratica keine Keimlinge gefunden, obwohl zumindest bei $A$. sparsifolia Diasporen in der Samenbank vorhanden waren (BRUELHEIDE, H., mündliche Mitteilung). Auf der Calligonum-Fläche, ebenso auf der in der Nähe liegenden Freifläche liefen große Mengen an C. caput-medusae-Keimlingen auf. Bereits wenige Wochen später waren allerdings keine lebenden Keimlinge mehr zu finden (eigene Beobachtungen). Die einmalige Bewässerung hat nicht genügend Wasser für die Etablierung von C. caput-medusae bereitgestellt. Einzig auf der Tamarix-Fläche konnten sich im Spätsommer 1999 gekeimte Tamarisken bis zum Frühjahr 2000 halten. Eine im April 2000 ausgegrabene Jungpflanze hatte schon 
eine etwa $40 \mathrm{~cm}$ lange Wurzel. Dann jedoch trat eine Käferkalamität auf, die keine der jungen $T$. ramosissima-Pflanzen überlebte. Es konnte daher nicht verfolgt werden, ob die Überflutung im August 1999 für die Etablierung ausgereicht hätte.

XIA et al. (1993) führen an, dass Calligonum-Arten im extrem ariden Gebiet der südlichen Taklamakan ohne Überflutung nicht überleben können. Dies konnte nicht bestätigt werden. Es muss vielmehr gesagt werden, dass eine Etablierung ohne Bewässerung nicht möglich ist, wohl aber das Überleben einmal etablierter Bestände, denn es konnten keine positiven Auswirkungen der Bewässerung auf den Wasserhaushalt festgestellt werden.

\subsection{Management-Empfehlungen}

Das Problem der Desertifikation wird in China schon seit langem diskutiert (z.B. ZHU \& LIU 1988, MAKI et al. 1993, TIAN \& SONG 1997), meist werden aber nur die Auswirkungen auf die Acker- und Weideflächen beschrieben. Die Wichtigkeit der Vorlandvegetation wird nur in den wenigsten Fällen betont (LIU et al. 1988), obwohl in vielen Veröffentlichungen darauf hingewiesen wird, dass die ländliche Bevölkerung die dort wachsenden Pflanzen nutzt (z.B. ZHANG \& MAO 1990).

Aufforstungsmaßnahmen werden seit den 1970er Jahren propagiert, bei diesen handelt es sich aber in der Regel um Anpflanzungen schnell wachsender Baumarten als Windschutz zwischen den Ackerflächen und nur selten um Arten, die von der Bevölkerung genutzt werden („multi-purpose trees“). Zudem wurden für die Anpflanzungen nicht immer einheimische, an Trockenheit angepasste Bäume verwendet (DREGNE 1988). Populus euphratica verträgt höhere Salzgehalte im Boden als z.B. Populus nigra oder andere häufig verwendete Baumarten und ist besser an Trockenstress angepasst (WANG et al. 1996). Außerdem steht $P$. euphratica auf der Liste der geschützten Arten in China, da die Auwälder in den nordwestchinesischen Trockengebieten durch Übernutzung der Wälder und Wasserressourcen drastisch zurückgegangen sind (PAN \& YING 1995).

DREGNE (1988) weist darauf hin, dass die in Xinjiang durchgeführten Anpflanzungen von Pappeln in vier- bis sechsreihigen Schutzgürteln zu erhöhtem 
Wasserverbrauch führen und außerdem noch nicht einmal die wandernden Dünen aufhalten, da die hohen Stämme keine Äste in Bodennähe aufweisen. Um die Felder vor Sanddrift zu schützen ist in jedem Fall ein vielschichtiges System aus angepflanzten Baumreihen und Büschen notwendig, das aber viel effektiver wird, wenn eine intakte Vorlandvegetation vorhanden ist (LIU et al. 1988, XIA et al. 1993). Im Oasenvorland von Qira wurde auf den Untersuchungsflächen Sandakkumulation von $4 \mathrm{~cm}$ auf der Alhagi-Fläche bis zu 7,5 cm auf der Tamarix-Fläche gemessen (BRUELHEIDE, H., unveröffentlicht). Auf kleinen mit $T$. ramosissima oder C. caput-medusae bewachsenen Dünen wurde sogar innerhalb eines Jahres eine Sandakkumulation von bis zu $1 \mathrm{~m}$ beobachtet (eigene Beobachtungen). Dünen, deren Vegetationsdecke zerstört wurde, weisen eine stärkere Sandbewegung auf. Tamarix-Dünen werden auf der Luvseite abgetragen, so dass alte Wurzelstöcke zutage treten, während sie auf der Leeseite Sand akkumulieren (eigene Beobachtungen). Die Abtragung der Dünen wird noch dadurch beschleunigt, dass die freigelegten Wurzelstöcke gerne als Brennholz gesammelt werden.

Ein Schutz der Vorlandvegetation ist also aus mehreren Gründen empfehlenswert: Als zusätzlicher Schutz der Ackerflächen vor Sanddrift ist das Vorland wichtig, als Winterweide und Quelle für Brennholz und Viehfutter für die Bevölkerung unentbehrlich. Eine nachhaltige Nutzung kann nur erfolgen, wenn einerseits die Menge der entnommenen Biomasse kontrolliert wird, andererseits die Regeneration der Vegetation gefördert wird. Dies kann in vielen Bereichen nur durch Anpflanzung erfolgen, da die natürliche Regeneration offenbar nur in Flusstälern stattfindet. Ein Weidemanagement mit Beweidungsausschluss für die Gebiete, in denen sich die Vegetation regenerieren soll, ist unumgänglich (siehe auch MITCHELL et al. 1998).

Da die untersuchten Arten alle vom Grundwasser abhängen, ist eine Kontrolle der Grundwasserstände empfehlenswert. In dieser Arbeit wurden nur geringe Schwankungen des Grundwasserspiegels auf den Untersuchungsflächen im Oasenvorland festgestellt. In den Brunnen der Oase treten aber laut Auskunft der örtlichen Behörden Schwankungen von etwa $4 \mathrm{~m}$ im Jahresverlauf auf. Es ist bisher unklar, wie weit sich diese Schwankungen ins Vorland erstrecken und ob es eine Absenkung des Grundwasserspiegels in den letzten Jahren gab. Obwohl die untersuchten Arten große Entfernung zum Grundwasser ertragen können, ist nicht 
bekannt, wie sie auf dessen Absenkung reagieren. HORTON et al. (2001) berichten von zunehmendem Absterben der Krone bei Tamarix chinensis (oft als Bezeichnung für die Gruppe aus $T$. chinensis, $T$. ramosissima und T. parviflora benutzt), wenn das Grundwasser auf unter $3 \mathrm{~m}$ sinkt. In dieser Untersuchung war T. ramosissima aber wohl nicht vom Grundwasser abhängig, sondern hauptsächlich an die Versorgung mit Oberflächenwasser angepasst, daher litt $T$. ramosissima dort in Trockenjahren auch bei einer Entfernung von nur 2-3 m zum Grundwasser unter Trockenstress.

Das großflächige Absterben der Populus euphratica Wälder am Unterlauf des Tarim und an anderen Flussläufen in Xinjiang wird auf sinkendes Grundwasser und verringerten Abfluss zurückgeführt (OVEZLIEV et al. 1993, TIAN \& SONG 1997). So beschreiben auch $\mathrm{Jl}$ et al. (2001), dass $P$. euphratica am Tarim bei Grundwasserständen von 5 bis $10 \mathrm{~m}$ abzusterben beginnt.

Eine Absenkung des Grundwassers sollte also vermieden werden, damit die bestehende Vegetation im Oasenvorland erhalten wird. Regenerationsmaßnahmen sind notwendig, um einen Schutz vor Sanddrift zu gewährleisten und eine nachhaltige Nutzung zu ermöglichen. 


\section{Zusammenfassung}

Am Südrand der Taklamakan-Wüste herrscht bei nur $33 \mathrm{~mm}$ Jahresniederschlag eine potentielle Evaporation von $2600 \mathrm{~mm}$. Die Oasen werden von Flüssen aus dem Kunlun-Gebirge gespeist, die zur Zeit der Schneeschmelze im Sommer Hochwasser führen. Zwischen Oase und Wüste liegt ein Gürtel natürlicher Vegetation, der als Schutz gegen Sanddrift sowie als Quelle für Viehfutter, Brennholz und Baumaterial dient. Am Beispiel der Oase Qira wurden der Wasserhaushalt und die Wassernutzungseffizienz von vier ökonomisch und ökologisch wichtigen Arten der Vorlandvegetation, Alhagi sparsifolia Shap., Tamarix ramosissima Lebed., Populus euphratica Oliv. sowie die $\mathrm{C}_{4}$-Art Calligonum caput-medusae Schrenk., auf zu diesem Zweck abgegrenzten Flächen untersucht. Die Auswirkungen einer experimentellen Bewässerung und einer simulierten Nutzung oberirdischer Pflanzenbiomasse auf den Wasserhaushalt der Arten wurden erforscht. Die Ergebnisse sollen dazu beitragen, ökologische Grundlagen für die Restauration und die nachhaltige Nutzung der Vegetation des Oasenvorlandes zu schaffen.

Die Pflanzen der Oasenvorlandvegetation hatten einen hohen Wasserverbrauch (Saftflusssumme der Vegetationsperiode 1999: C. caput-medusae $183 \mathrm{~kg} \mathrm{H}_{2} \mathrm{O}^{*} \mathrm{~m}^{-2}$, A. sparsifolia $391 \mathrm{~kg} \mathrm{H}_{2} \mathrm{O}^{*} \mathrm{~m}^{-2}, T$. ramosissima bis zu $441 \mathrm{~kg} \mathrm{H}_{2} \mathrm{O}^{*} \mathrm{~m}^{-2}$; für $P$. euphratica liegt ein mit dem PENMAN-MONTEITH-Ansatz berechneter Wert von $392 \mathrm{~kg} \mathrm{H}_{2} \mathrm{O}^{*} \mathrm{~m}^{-2}$ vor), den sie nur aus dem Grundwasser decken können, da die Bodenwassergehalte außerhalb von Überflutungsperioden bis in größere Tiefen vernachlässigbar gering sind und der größte Teil des Vorlandes nicht von den Sommerüberflutungen erreicht wird. Die Wasserpotentiale deuteten nicht auf starken Trockenstress hin und weder Transpiration noch Saftfluss wiesen eine mittägliche Einschränkung auf. Eine experimentelle Bewässerung hatte keinen positiven Effekt auf den Wasserhaushalt der untersuchten Arten. Die verbrauchten Wassermengen waren auf den unbewässerten Flächen niedriger als auf den bewässerten Flächen. Die Produktivität war bei $A$. sparsifolia und $C$. caputmedusae auf den unbewässerten Flächen höher, bei $T$. ramosissima und $P$. euphratica niedriger als auf den bewässerten Flächen. Dies war aber nicht auf einen Bewässerungseffekt zurückzuführen. Zwischen den unbewässerten und bewässerten Untersuchungsflächen existierten prinzipielle Unterschiede, die sich 
auf den Wasserhaushalt und die Produktivität auswirkten: bei $C$. caput-medusae und $T$. ramosissima konnten diese Unterschiede auf eine größere Entfernung der Pflanzen auf den unbewässerten Flächen zum Grundwasser zurückgeführt werden; bei $P$. euphratica und vielleicht auch bei $A$. sparsifolia, beides Arten mit klonalem Wachstum, liegen vermutlich genetische Unterschiede zwischen den Flächen vor. Die experimentelle Nutzung durch Ernte oberirdischer Biomasse wirkte sich bei $A$. sparsifolia negativ auf die Produktion im folgenden Jahr, bei den anderen Arten positiv auf die Produktivität der verbleibenden Triebe aus.

Aus der Produktivität und dem Jahreswasserverbrauch aus Saftflussmessungen wurde die Wassernutzungseffizienz der Produktion (WUEp) errechnet. Die untersuchten Arten hatten eine sehr hohe Produktion im Vergleich mit Arten in anderen Trockengebieten. Die WUEp war bei der $\mathrm{C}_{4}$-Pflanze $C$. caput-medusae mit 1,16 $\mathrm{g} \mathrm{TG}^{*} \mathrm{~kg} \mathrm{H}_{2} \mathrm{O}^{-1}$ am höchsten, bei $A$. sparsifolia mit 0,60 $\mathrm{g} \mathrm{TG}^{*} \mathrm{~kg} \mathrm{H}_{2} \mathrm{O}^{-1}$ am niedrigsten. $T$. ramosissima erreichte $0,82 \mathrm{~g} \mathrm{TG}^{*} \mathrm{~kg} \mathrm{H}_{2} \mathrm{O}^{-1}$. Die Werte für die WUEp liegen insgesamt sehr niedrig im allgemeinen Artvergleich. Die Kohlenstoffisotopenverhältnisse $\left(\delta^{13} \mathrm{C}\right)$ zeigten einen Jahresverlauf, der unabhängig von der Bewässerung absank, was auf metabolische Prozesse zurückzuführen ist. Weder eine Einschränkung der Wasserverfügbarkeit im Jahresverlauf noch ein positiver Bewässerungseffekt konnte auf Grundlage dieser Ergebnisse angenommen werden.

Eine natürliche Regeneration der Vorlandvegetation erfolgt offenbar nur in Flussnähe, für den Großteil der Fläche müssten Anpflanzungen und Bewässerung erfolgen. Eine Regeneration kann nur erfolgreich sein, wenn die Pflanzen das Grundwasser erreichen. C. caput-medusae ist als Art mit der höchsten Wassernutzungseffizienz für Pflanzungen zur Dünenbefestigung zu empfehlen, allerdings dürfen die Nutzungsinteressen der lokalen Bevölkerung nicht außer Acht gelassen werden: A. sparsifolia ist eine wichtige Futterpflanze, $T$. ramosissima als Holzlieferant mit hoher Produktivität bedeutend; auch diese Arten sollten daher gefördert werden. $P$. euphratica als geschützte Art sollte gefördert werden bis sich Bestände erholt haben; prinzipiell sind Populus-Bestände eine bedeutende Ressource für Holz und könnten zudem als innerer Schutzgürtel um die Oasen einen Beitrag zur Verhinderung von Sandverwehung leisten. Ein Managementplan für Beweidung und Nutzung des Vorlandes ist Voraussetzung für eine nachhaltige 
Nutzung. Eine intakte Vorlandvegetation zusammen mit angepflanzten Schutzgürteln bietet den effektivsten Schutz gegen Sanddrift.

\section{Summary}

At the southern fringe of the Taklamakan desert, the annual precipitation is only $33 \mathrm{~mm}$, and the potential evaporation $2600 \mathrm{~mm}$. The oases are supplied with water through rivers, which run from the Kunlun Mountains and carry high water during snow melt in summer. A belt of natural vegetation marks the transition zone between oasis and desert, and serves as a shelter against sand drift and as a source of fodder, firewood and construction material. Taking the oasis Qira as an example, the four key species of the foreland vegetation: Alhagi sparsifolia Shap., Tamarix ramosissima Lebed., Populus euphratica Oliv. and the $\mathrm{C}_{4}$ species Calligonum caput-medusae Schrenk. were investigated on their water status and water use efficiency. The studies were conducted at fenced research sites that were dominated by one of these plant species each. The effects of experimental irrigation and of harvest of above-ground biomass (to mimic the use of biomass by the local farmers) on the water status of the species was explored. The results should contribute to create the ecological basis for the restoration and sustainable use of the foreland vegetation.

The plants of the foreland vegetation exhibited a high water use (sap flow sum of the vegetation period of 1999: C. caput-medusae, $183 \mathrm{~kg} \mathrm{H}_{2} \mathrm{O}^{*} \mathrm{~m}^{-2} ;$ A. sparsifolia, $391 \mathrm{~kg} \mathrm{H}_{2} \mathrm{O}^{*} \mathrm{~m}^{-2}$; $T$. ramosissima, up to $441 \mathrm{~kg} \mathrm{H}_{2} \mathrm{O}^{*} \mathrm{~m}^{-2}$; for $P$. euphratica a value of $392 \mathrm{~kg} \mathrm{H}_{2} \mathrm{O}^{*} \mathrm{~m}^{-2}$ was calculated using the PENMAN-MONTEITH equation). This high demand of water can only be met by ground water use, as the soil water content is negligible except for flooding periods, and as most of the foreland area is not reached by natural summer floods. The water potentials did not indicate severe drought stress, and neither transpiration nor sap flow were impaired during midday. An experimental irrigation did not have any positive effect on the water status of the investigated species. On the non-irrigated plots, water use was lower than on their irrigated counterparts. Compared to the irrigated plots, productivity was higher on the non-irrigated plots of $A$. sparsifolia and C. caput-medusae, but lower on those of $T$. ramosissima and $P$. euphratica. These discrepancies were not due to irrigation but to general differences between the research plots that affected water 
use and productivity: in C. caput-medusae and T. ramosissima, these differences were due to a longer distance to ground water on the non-irrigated plots; in $P$. euphratica and maybe also in $A$. sparsifolia, both being species with a pronounced clonal growth, genetic differences between plant clusters (genets) on the respective plots may have been effective. The harvest of above-ground biomass had a negative effect on productivity of $A$. sparsifolia in the following year, but a positive effect on productivity of the remaining shoots of the other species.

Water use efficiency of production (WUEp) was calculated from productivity and annual water use measured by sap flow. Compared to plants in other arid regions, the investigated species exhibited a high productivity. The $\mathrm{C}_{4}$ species $C$. caputmedusae showed the highest WUEp $\left(1,16 \mathrm{~g} \mathrm{DM}^{*} \mathrm{~kg} \mathrm{H}_{2} \mathrm{O}^{-1}\right)$, whereas WUEp of $A$. sparsifolia was lowest $\left(0,60 \mathrm{~g} \mathrm{DM}^{*} \mathrm{~kg} \mathrm{H}_{2} \mathrm{O}^{-1}\right)$. T. ramosissima attained $0,82 \mathrm{~g} \mathrm{DM}^{*} \mathrm{~kg} \mathrm{H}_{2} \mathrm{O}^{-1}$. These WUEp values are rather low in comparison to other species. Irrespective of irrigation, the carbon isotope ratio $\left(\delta^{13} \mathrm{C}\right)$ showed a decline through the vegetation period that is due to metabolic processes. On the basis of these results, neither a restricted water availability throughout the year nor a positive effect of irrigation on the water status of the plants could be assumed.

Apparently, a natural regeneration of the foreland vegetation takes place only near riverbeds. Therefore, for the restoration of most of the foreland area, plantings and irrigation are necessary. Regeneration measures can only be successful if the plants are able to gain access to ground water. Being the species with the highest water use efficiency, $C$. caput-medusae is recommended for sand fixation. However, the needs of the local population have to be considered: $A$. sparsifolia is a valuable fodder plant, and $T$. ramosissima is important as a highly productive source of wood for fuel and construction material; therefore, these species should be promoted as well. $P$. euphratica as a protected species should be fostered as well; once the stands have recovered, they represent a valuable resource of wood and a shelterbelt around the oases that can contribute to the prevention of sand drift. A management plan for grazing and use of the foreland vegetation is a prerequisite for a sustainable development. An intact foreland vegetation in combination with planted shelterbelts offers the most effective shelter against sand drift. 


\section{Literaturliste}

Alarcón,J.J., Domingo,R., Green,S.R., Sánchez-Blanco,M.J., Rodríguez,P., and Torrecillas,A. (2000) Sap flow as an indicator of transpiration and the water status of young apricot trees. Plant and Soil 227: 77-85.

Allen,R.G., Pereira,L.S., Raes,D., and Smith,M. (1998) Crop evapotranspiration Guidelines for computing crop water requirements. Rom: FAO- Food and Agriculture Organization of the United Nations.

Allen,S.J. and Grime,V.L. (1995) Measurements of transpiration from savannah shrubs using sap flow gauges. Agricultural and Forest Meteorology 75: 23-41.

Améglio,T., Archer,P., Cohen,M., Valancogne,C., Daudet,F.A., Dayau,S., and Cruiziat,P. (1999) Significance and limits in the use of predawn leaf water potential for tree irrigation. Plant and Soil 207: 155-167.

Anderson,J.E. (1982) Factors Controlling Transpiration and Photosynthesis in Tamarix chinensis Lour. Ecology 63: 48-56.

Ansley,R.J., Dugas,W.A., Heuer,M.L., and Trevino,B.A. (1994) Stem flow and porometer measurements of transpiration from honey mesquite (Prosopis glandulosa). Journal of Experimental Botany 45: 847-856.

Arampatsis,C. (2001) Ökophysiologische Untersuchungen zur Habitatanpassung der Wüstenpflanze Alhagi sparsifolia. Universität Wien: Diplomarbeit, Institut für Ökologie und Naturschutz, Abt. Chemische Pflanzenphysiologie.

Arndt,S.K., Wanek,W., Clifford,S.C., and Popp,M. (2000a) Contrasting adaptations to drought stress in field-grown Ziziphus mauritiana and Prunus persica trees: water relations, osmotic adjustment and carbon isotope composition. Australian Journal of Plant Physiology 27: 985-996.

Arndt,S.K., Foetzki,A., Thomas,F.M., and Popp,M. (2000b) Are plants in the Chinese Taklamakan desert water limited? A stable isotope approach. Proceedings of the FAO/IAEA International Symposium on Nuclear Techniques in Integrated Plant Nutrient, Water and Soil Management, Vienna, Austria, Oct. 16-20, 2000.

Arndt,S.K., Clifford,S.C., Wanek,W., Jones,H.G., and Popp,M. (2001) Physiological and morphological adaptations of the fruit tree Ziziphus rotundifolia in response to progressive drought stress. Tree Physiology 21: 705-715.

Arndt,S.K., Arampatsis,C., Foetzki,A., Li,X., Zeng,F., and Zhang,X. (2002) Accumulation of leaf solutes during the vegetation period in phreatophytic desert plants growing in a hyperarid desert with saline groundwater. J Arid Environ eingereicht.

Backes,K. (1996) Der Wasserhaushalt vier verschiedener Baumarten der Heide-WaldSukzession. 134 pp., Dissertation, Georg-August-Universität Göttingen.

Baum,B.R. (1978) The Genus Tamarix. Jerusalem: The Israel Academy of Sciences and Humanities. 
Bréda,N., Cochard,H., Dreyer,E., and Granier,A. (1993) Water transfer in a mature oak stand (Quercus petraea): seasonal evolution and effects of a severe drought. Canadian Journal of Forest Research 23: 1136-1143.

Bréda,N., Granier,A., and Aussenac,G. (1995) Effects of thinning on soil and tree water relations, transpiration and growth in an oak forest (Quercus petraea (Matt.) Liebl.). Tree Physiology 15: 295-306.

Brotherson,J.D. and Winkel,V. (1986) Habitat relationships of saltcedar (Tamarix ramosissima) in central Utah. Great Basin Naturalist 46: 535-541.

Bruelheide,H., Foetzki,A., Gries,D., Thomas,F.M., and Runge,M. (2001) Individual Partner Report: Albrecht-von-Haller-Institute for Plant Sciences, Dept. Ecology and Ecosystem Research, University of Göttingen. In Final Project Report - Ecological Basis for a Sustainable Management of the Indigenous Vegetation in a Central Asian Desert. INCO-DC: ERBIC18CT980275. Runge,M. (ed).

Bruelheide,H., Jandt,U., Gries,D., Thomas,F.M., Foetzki,A., Buerkert,A., Wang,G., Zhang, $X$., Runge,M. (2003) Vegetation changes in a river oasis on the southern rim of the Taklamakan Desert in China between 1956 and 2000. Phytocoenologia 33: 801818.

Burgess,S.S.O., Adams,M.A., Turner,N.C., and Ong,C.K. (1998) The redistribution of soil water by tree root systems. Oecologia 115: 306-311.

Burgess,S.S.O., Pate,J.S., Adams,M.A., and Dawson,T.E. (2000) Seasonal Water Acquisition and Redistribution in the Australian Woody Phreatophyte, Banksia prionotes. Annals of Botany 85: 215-224.

Burgess,S.S.O., Adams,M.A., Turner,N.C., White,D.A., and Ong,C.K. (2001) Tree roots: conduits for deep recharge of soil water. Oecologia 126: 158-165.

Busch,D.E. and Smith,S.D. (1993) Effects of fire on water and salinity relations of riparian woody taxa. Oecologia 94: 186-194.

Caldwell,M.M., Dawson,T.E., and Richards,J.H. (1998) Hydraulic lift: consequences of water efflux from the roots of plants. Oecologia 113: 151-161.

Canadell,J., Jackson,R.B., Ehleringer,J.R., Mooney,H.A., Sala,O.E., and Schulze,E.D. (1996) Maximum rooting depth of vegetation types at the global scale. Oecologia 108: 583-595.

Cerling,T.E. (1999) Paleorecords of $\mathrm{C}_{4}$ Plants and Ecosystems. In $C_{4}$ Plant Biology. Sage,R.F. and Monson,R.K. (eds). San Diego London: Academic Press, 445-469.

Cheng,H. (1991a) Impact of water on the eco-geographic environment along the Keriya River. Die Erde Erg.-H.6: 148-164.

Cheng,H. (1991b) The change of eco-environment and the rational utilization of water resources in the Keriya River Valley. Die Erde Erg.-H. 6: 133-147.

Cheng,Z.C. (1997) A catastrophic flood in 1994 and some relative problems in the Tarim River. Chinese Journal of Arid Land Research 10: 207-216.

Cleverly,J.R., Smith,S.D., Sala,A., and Devitt,D.A. (1997) Invasive capacity of Tamarix ramosissima in a Mojave desert floodplain: the role of drought. Oecologia 111: 1218. 
Clifton-Brown,J.C. and Lewandowski,I. (2000) Water Use Efficiency and Biomass Partitioning of Three Different Miscanthus Genotypes with Limited and Unlimited Water Supply. Annals of Botany 86: 191-200.

Cohen,Y., Adar,E., Dody,A., and Schiller,G. (1997) Underground water use by Eucalyptus trees in an arid climate. Trees 356-362.

Comstock,J.P. and Ehleringer,J.R. (1992) Correlating genetic variation in carbon isotopic composition with complex climatic gradients. Proceedings National Academy of Sciences, U. S. A. 89: 7747-7751.

Comstock,J.P. and Mencuccini,M. (1998) Control of stomatal conductance by leaf water potential in Hymenoclea salsola (T. \& G.), a desert subshrub. Plant Cell Environ 21: 1029-1038.

Comstock,J.P. (2002) Hydraulic and chemical signalling in the control of stomatal conductance and transpiration. Journal of Experimental Botany 53: 195-200.

Coners,H. (2001) Wasseraufnahme und artspezifische hydraulische Eigenschaften der Feinwurzeln von Buche, Eiche und Fichte: In situ-Messungen an Altbäumen. Dissertation, Georg-August-Universität Göttingen.

Coque,R., Gentelle,P., and Coque-Delhuille,B. (1991) Desertification along the piedmont of the Kunlun Chain (Hetian-Yutian sector) and the southern border of the Taklamakan Desert (China): Preliminary geomorphological observations (1). Revue de Géomorphologie Dynamique XL 4: 1-27.

Damesin,C., Rambal,S., and Joffre,R. (1997) Between-tree variations in leaf $\delta^{13} \mathrm{C}$ of Quercus pubescens and Quercus ilex among Mediterranean habitats with different water availability. Oecologia 111: 26-35.

David,T.S., Ferreira,M.I., David,J.S., and Pereira,J.S. (1997) Transpiration from a mature Eucalyptus globulus plantation in Portugal during a spring-summer period of progressively higher water deficit. Oecologia 110: 153-159.

Devitt,D.A., Morris,R.L., and Neumann,D.S. (1994) Evapotranspiration and growth response of three woody ornamental species placed under varying irrigation regimes. Journal of the American Society of Horticultural Sciences 119: 452-457.

Devitt,D.A., Sala,A., Mace,K.A., and Smith,S.D. (1997) The effect of applied water on the water use of saltcedar in a desert riparian environment. Journal of Hydrology 192: 233-246.

Devitt,D.A., Smith,S.D., and Neumann,D.S. (1997) Leaf carbon isotope ratios in three landscape species growing in an arid environment. J Arid Environ 36: 249-257.

Devitt,D.A. and Smith,S.D. (2002) Root channel macropores enhance downward movement of water in a Mojave Desert ecosystem. J Arid Environ 50: 99-108.

Di Tomaso,J.M. (1998) Impact, biology, and ecology of saltcedar (Tamarix spp.) in the southwestern United States. Weed Technology 12: 326-336.

Donovan,L.A. and Ehleringer,J.R. (1992) Contrasting water-use patterns among size and life-history classes of a semi-arid shrub. Functional Ecology 6: 482-488. 
Donovan,L.A., Grisé,D.J., West,J.B., Pappert,R.A., Alder,N.N., and Richards,J.H. (1999) Predawn disequilibrium between plant and soil water potentials in two cold-desert shrubs. Oecologia 120: 209-217.

Dregne,H.E. (1978) Desertification: man's abuse of the land. Journal of Soil and Water Conservation 33: 11-14.

Dregne,H.E. (1988) Irrigation development in northwestern Xinjiang. Chinese Journal of Arid Land Research 1: 185-190.

Edwards,T.W.D., Graf,W., Trimborn,P., Stichler,W., Lipp,J., and Payer,H.D. (2000) $\delta^{13} \mathrm{C}$ response surface resolves humidity and temperature signals in trees. Geochimica and Cosmochimica Acta 64: 161-167.

Ehdaie,B., Hall,A.E., Farquhar,G.D., Nguyen,H.T., and Waines,J.G. (1991) Water-use efficiency and carbon isotope discrimination in wheat. Crop Science 31: 12821288.

Ehleringer,J.R., Phillips,S.L., and Comstock,J.P. (1992) Seasonal variation in the carbon isotopic composition of desert plants. Functional Ecology 6: 396-404.

Ellenberg,H., Mayer,R., and Schauermann,J. (1986) Ökosystemforschung - Ergebnisse des Solling-Projekts 1966-1986. Stuttgart: Ulmer.

Everitt,B.L. (1980) Ecology of Saltcedar - A Plea for Research. Environ Geo/ 3: 77-84.

Fan,Z. (1995) Eco-Environmental Change Under Human Impact in Xinjiang. Chinese Journal of Arid Land Research 8: 7-10.

Farquhar,G.D., O'Leary,M.H., and Berry,J.A. (1982) On the relationship between carbon isotope discrimination and the intercellular carbon dioxide concentration in leaves. Australian Journal of Plant Physiology 9: 121-137.

Farquhar,G.D. and Richards,R.A. (1984) Isotopis composition of plant carbon correlates with water-use efficiency of wheat genotypes. Australian Journal of Plant Physiology 11: 539-552.

Farquhar,G.D., Ehleringer,J.R., and Hubick,K.T. (1989) Carbon isotope discrimination and photosynthesis. Annu. Rev. Plant Physiol. Plant Mol. Biol. 40: 503-537.

Fimkin,V.P., Makhzumova,M.I., Daurenbekova,L.T., and Khusanov,A.A. (1990) Cultivation of planting material of desert shrubs for phytomelioration of deserts. Problems of Desert Development 4: 60-63.

Gebauer,R.L.E. and Ehleringer,J.R. (2000) Water and nitrogen uptake patterns following moisture pulses in a cold desert community. Ecology 85: 1415-1424.

Gibbens,R.P. and Lenz,J.M. (2001) Root systems of some Chihuauan Desert plants. J Arid Environ 49: 221-263.

Gladwin,D.N. and Roelle,J.E. (1998) Survival of plains cottonwood (Populus deltoides subsp. monilifera ) and saltcedar (Tamarix ramosissima) seedlings in response to flooding. Wetlands 18: 669-674.

Gleixner,G., Scrimgeour,C., Schmidt,H.-L., and Viola,R. (1998) Stable isotope distribution in the major metabolites of source and sink organs of Solanum tuberosum L.: a 
powerful tool in the study of metabolic partitioning in intact plants. Planta 207: 241245.

Glenn,E., Tanner,R., Mendez,S., Kehret,T., Moore,D., Garcia,J., and Valdes,C. (1998) Growth rates, salt tolerance and water use characteristics of native and invasive riparian plants from the delta of the Colorado River, Mexico. J Arid Environ 40: 281294.

Gries,D., Foetzki,A., Arndt,S.K., Bruelheide,H., Thomas,F.M., Zhang,X., and Runge,M. Allometric Production Estimates of the Foreland Vegetation of a Taklamakan Desert River Oasis. Plant Ecology eingereicht.

Gries,D., Zeng,F., Foetzki,A., Arndt,S.K., Bruelheide,H., Thomas,F.M. et al. (2003) Growth and water relations of Tamarix ramosissima and Populus euphratica on Taklamakan desert dunes in relation to depth to a permanent water table. Plant Cell Environ 26: 725-736.

Griffiths,H. (1998) Stable isotopes: integration of biological, ecological and geochemical processes. Oxford: BIOS Scientific Publishers.

Haase, R. and Haase,P. (1995) Above-ground biomass estimates for invasive trees and shrubs in the Pantanal of Mato Grosso, Brazil. Forest Ecology and Management 73: 29-35.

Hedin,S. (1899) Durch Asiens Wüsten. Leipzig.

Hinckley,T.M., Duhme,F., Hinckley,A.R., and Richter,H. (1980) Water relations of drought hardy shrubs: osmotic potential and stomatal reactivity. Plant, Cell and Environment 3: 131-140.

Horton,J.L., Kolb,T.E., and Hart,S.C. (2001) Responses of riparian trees to interannual variation in ground water depth in a semi-arid river basin. Plant, Cell and Environment 24: 293-304.

Horton,J.S. and Campbell,C.J. (1974) Management of Phreatophyte and Riparian Vegetation for Maximum Multiple Use Values. United States Forest Service Research Paper 117: 1-23.

Hoyanagi,M. (1965) Sand-buried Ruins and Shrinkage of Rivers along the Old Silk Road Region in the Tarim Basin (I). J. Geography Tokyo 74: 1-12.

Hövermann,J. (1988) Die 1. Chinesisch-Deutsche Kuen-Luen-Taklamakan-Expedition. Braunschweiger Wiss. Ges. Jahrb. 1988: 11-16.

Hsiao,T.C. and Läuchli,A. (1986) Role of potassium in plant-water relations. In Advances in plant nutrition. Vol. 2. Tinker,B. and Läuchli,A. (eds). New York: Praeger, 281312.

Hubbard,R.M., Bond,B.J., and Ryan,M.G. (1999) Evidence that hydraulic conductance limits photosynthesis in old Pinus ponderosa trees. Tree Physiology 19: 165-172.

Hubbard,R.M., Ryan,M.G., Stiller,V., and Sperry,J.S. (2001) Stomatal conductance and photosynthesis vary linaerly with plant hydraulic conductance in ponderosa pine. Plant Cell Environ 24: 113-121.

Hudaberdi,M. (2000) Tamaricaceae. In Claves Plantarum Xinjiangensis. Urumqi: Xinjiang University Publishing House, 339-340. 
Jäkel,D. (1990) Desertifikation und Maßnahmen zur Dünenfixierung in China. Berliner Geographische Abhandlungen 53: 139-148.

Ji,F., Ma,Y.J., and Fan,Z.L. (2001) Soil water regime in Populus euphratica forest on the Tarim River alluvial plain. Acta Phytoecologica Sinica 25: 17-21.

Jin,Q.H. (2002) Alhagi sparsifolia Schap.: A potentially utilizable forage in saline soils. In Halophytes as a resource for livestock and for rehabilitation of degraded lands. Squires, V.R. and Ayoub,A.T. (eds). Kluwer Academic Publishers, 285-288.

Kassas,M. (1952) On the reproductive capacity and life cycle of Alhagi maurorum. Proceedings of the Egyptian Academy of Sciences 8: 114-122.

Katyal,J.C. and Vlek,P.L.G. (2000) Desertification - Concept, Causes and Amelioration. ZEF - Discussion Papers On Development Policy 33.

Keller,B. (1925) Halophyten- und Xerophyten-Studien. Journal of Ecology 13: 224-261.

Kerr,H.D., Robocker,W.C., and Muzik,T.J. (1965) Characteristics and Control of Camelthorn. Weeds 13: 156-163.

Kleinkopf,G.E. and Wallace,A. (1974) Physiological basis for salt tolerance in Tamarix ramosissima. Plant Science Letters 3: 157-163.

Koide,R.T., Robichaux,R.H., Morse,S.R., and Smith,C.M. (1989) Plant water status, hydrau-lic resistence and capacitance. In Plant Physiological Ecology - field methods and instrumentation. Pearcy,R.W., Ehleringer,J.R., Mooney,H.A., and Rundel,P.W. (eds). 161-183.

Köstner,B., Granier,A., and Cermák,J. (1998) Sapflow measurements in forest stands: methods and uncertainties. Ann. Sci. For. 55: 13-27.

Kurban,H., Saneoka,H., Nehira,K., Adilla,R., and Fujita,K. (1998) Effect of Salinity on Growth and Accumulation of Organic and Inorganic Solutes in the Leguminous Plants Alhagi pseudoalhagi and Vigna radiata. Soil Sci. Plant Nutr. 44: 589-597.

Kurban,H., Saneoka,H., Nehira,K., Adilla,R., Premachandra,G., and Fujita,K. (1999) Effect of Salinity on Growth, Photosythesis and Mineral Composition in Leguminous Plant Alhagi pseudoalhagi (Bieb.). Soil Sci. Plant Nutr. 50: 851-862.

Lalymenko,N.K. and Lalymenko,L.A. (1990) Euphrates poplar in protective afforestation on sand soils irrigated with sewage. Problems of Desert Development 4: 64-67.

Lambers,H., Chapin III,F.S., and Pons,T.L. (1998) Plant Physiological Ecology. New York: Springer.

Larcher,W. (1994) Ökophysiologie der Pflanzen: Leben, Leistung und Streßbewältigung der Pflanzen in ihrer Umwelt. Stuttgart: Verlag Eugen Ulmer.

Laundré,J.W. (1999) Relationships between water availability, carbon isotope discrimination and plant productivity in two semi-arid grass and shrub species. $J$ Arid Environ 41: 49-60.

Le Houérou,H.N. (1996) Climate change, drought and desertification. J Arid Environ 34: 133-185. 
Leffler,A.J. and Evans,A.S. (2001) Physiological variation among Populus fremontii populations: short- and long-term relationships between $\delta^{13} \mathrm{C}$ and water availability. Tree Physiology 21: 1149-1155.

Liu,X.J. (1984) Experimental nursery operation of Tamarix ramosissima. Forest Science and Technology 10: 8-9.

Liu,X.M., Wang,Z.X., Tang,Z.Z., and Zhao,Y.F. (1988) The integrated transformation of desertified land in peripheral districts of an oasis. A case study of the northern part of the Linze oasis, Hexi Corridor. Chinese Journal of Arid Land Research 1: 271281.

Lozan,J.L. (1992) Angewandte Statistik für Naturwissenschaftler. Berlin, Hamburg: Parey.

López Villagra,G.M. and Felker,P. (1997) Influence of understory removal, thinning and P fertilization on $\mathrm{N}_{2}$ fixation in a mature mesquite (Prosopis glandulosa var. glandulosa) stand. J Arid Environ 36: 591-610.

Ma,H.C., Fung,L., Wang,S.S., Altman,A., Huttermann,A., and Jiang,X.N. (1996) Preliminary study on the salt resistance mechanisms of [seedlings of] Populus euphratica. Journal of Beijing Forestry University 5: 31-40.

Ma,H.C., Fung,L., Wang,S.S., Altman,A., and Hüttermann,A. (1997) Photosynthetic response of Populus euphratica to salt stress. Forest Ecology and Management 93: 55-61.

Mackerras,C. (2001) Xinjiang at the turn of the century: the causes of separatism. Central Asian Survey 20: 289-303.

Maillard,P., Deleens,E., Daudet,F.A., Lacointe,A., and rossard,J.S. (1994) Carbon and nitrogen partitioning in walnut seedlings during the acquisition of autotrophy through simultaneous ${ }^{13} \mathrm{CO}_{2}$ and ${ }^{15} \mathrm{NO}_{3}$ long term labelling. Journal of Experimental Botany 45: 203-210.

Maki,T., Pan,B., Du,M.Y., and Uemura,K. (1993) Effects of double line windbreaks [Tamarix chinensis] on the microclimate, sand accumulation and crop [cotton and sorghum] on arid land in Turfan [Xinjiang], China. Journal of Agricultural Meteorology 49: -255.

Mensching,H.G. (1990) Desertifikation. Ein weltweites Problem der ökologischen Verwüstung in den Trockengebieten der Erde. Darmstadt: Wissenschaftliche Buchgesellschaft.

Middleton,N.J. and Thomas,D.S. (1997) World Atlas of Desertification. London: Arnold.

Mitchell,D.J., Fullen,M.A., Trueman,I.C., and Fearnehough,W. (1998) Sustainability of reclaimed desertified land in Ningxia, China. J Arid Environ 39: 239-251.

Moers,W. (1999) Die 13 1/2 Leben des Käptn Blaubär. München: Goldmann.

Monteith,J.L. and Unsworth,M.H. (1990) Principles of environmental physics. London: Arnold.

Morecroft,M.D. and Woodward,F.I. (1990) Experimental investigations on the environmental determination of $\delta^{13} \mathrm{C}$ at different altitudes. Journal of Experimental Botany 41: 1303-1308. 
Nardini,A. and Salleo,S. (2000) Limitation of stomatal conductance by hydraulic traits: sensing or preventing xylem cavitation? Trees 15: 24.

Newton,A.C., Dick,J.M., and Heaton,T.H.E. (1996) Stable isotope composition $\left(\delta^{13} C\right)$ of Acacia tortilis subsp. spirocarpa (A. Rich.) Brenan growing at three semi-arid sites in Kenya. J Arid Environ 34: 325-330.

Nilsen,E.T., Sharifi,M.R., Rundel,P.W., Jarrel,W.M., and Virginia,R.A. (1983) Diurnal and seasonal water relations of the desert phreatophyt Prosopis glandulosa (honey mesquite) in the Sonoran desert of California. Ecology 64: 1381-1393.

Nobel,P.S. and Linton,M.J. (1997) Frequencies, Microclimate and Root Properties for Three Codominant Perennials in the Northwestern Sonoran Desert on North- vs. South-facing Slopes. Annals of Botany 80: 731-739.

Novikova,S.S. (1987) Vegetation of dry river beds of the ancient lli River delta. Problems of Desert Development 3: 22-29.

O'Leary,M.H. (1988) Carbon isotopes in photosynthesis. Bioscience 38: 325-336.

Oren,R., Sperry,J.S., Katul,G.G., Pataki,D.E., Ewers,B.E., Phillips,N., and Schäfer,K.V.R. (1999) Survey and synthesis of intra- and interspecific variation in stomatal sensitivity to vapour pressure deficit. Plant Cell Environ 22: 1515-1526.

Ovezliev,A.O., Kurbanov,O.R., and Liu,G.Z. (1993) Prospects of forest amelioration in arid zone of Xinjiang-Uigar Autonomous Region of China. Problems of Desert Development 1: 16-21.

Pan,B. and Ying,L. (1995) Comprehensive evaluation of rare and endangered plant species in the arid desert zones of China. Chinese Journal of Arid Land Research 8: $141-148$.

Pearcy,R.W., Schulze,E.D., and Zimmermann,R. (1989) Measurement of transpiration and leaf conductance. In Plant physiological ecology: field methods and instrumentation. Pearcy,R.W., Ehleringer,J.R., Mooney,H.A., and Rundel,P.W. (eds). London: Chapman and Hall, 137-160.

Pockman,W.T., Sperry,J.S., and O'Leary,J.W. (1995) Sustained and significant negative water pressure in xylem. Nature 378: 715-716.

Prior,L.D., Eamus,D., and Duff,G.A. (1997) Seasonal Trends in Carbon Assimilation, Stomatal Conductance, Pre-dawn Leaf Water Potential and Growth in Terminalia ferdinandiana, a Decidous Tree of Northern Australian Savannas. Australian Journal of Botany 45: 53-69.

Pyankov,V.I., Gunin,P.D., Tsoog,S., and Black,C.C. (2000) $\mathrm{C}_{4}$ plants in the vegetation of Mongolia: their natural occurence and geographical distribution in relation to climate. Oecologia 123: 15-31.

Qong,M., Takamura,H., and Hudaberdi,M. (2002) Formation and internal structure of Tamarix cones in the Taklimakan Desert. J Arid Environ 50: 81-97.

Richardson,J.M. (1953) Camelthorn (Alhagi camelorum Fisch.). Journal of the Department of Agriculture South Australia 57: 18-20.

Robinson,T.W. (1958) Phreatophytes. Washington: United States Government Printing Office. 
Sage,R.F. and Monson,R.K. (1999) C 4 Plant Biology. San Diego London: Academic Press.

Sala,A., Smith,S.D., and Devitt,D.A. (1996) Water use by Tamarix ramosissima and associated phreatophytes in a Mojave Desert floodplain. Ecological Applications 6: 888-898.

Saliendra,N.Z., Sperry,J.S., and Comstock,J.P. (1995) Influence of leaf water status on stomatal response to humidity, hydraulic conductance, and soil drought in Betula occidentalis. Planta 196: 357-366.

Schäfer,K.V.R., Oren,R., and Tenhunen,J.D. (2000) The effect of tree height on crown level stomatal conductance. Plant Cell Environ 23: 365-375.

Schenk,H.J. and Jackson,R.B. (2002) Rooting depths, lateral root spreads and belowground/above-ground allometries of plants in water-limited ecosystems. Journal of Ecology 90: 480-494.

Schneider,U. (1993) Phytomass, Element and Water Relations of Wild and Cultivated Plants in the Western Desert of SW Egypt. Catena Supplement 26: 179-199.

Scholander,P.F., Hammel,H.T., Bradstreet,E.D., and Hemmingsen,E.A. (1965) Sap pressure in vascular plants. Science 148: 339-346.

Schopfer,P. and Brennicke,A. (1999) Pflanzenphysiologie. Berlin Heidelberg: Springer.

Schulze,E.D., Caldwell,M.M., Canadell,J., Mooney,H.A., Jackson,R.B., Parson,D. et al. (1998) Downward flux of water through roots (i.e. inverse hydraulic lift) in dry Kalahari sands. Oecologia 115: 460-462.

Schulze,E.D., Williams,R.J., Farquhar,G.D., Schulze,W., Langridge,J., Miller,J.M., and Walker,B.H. (1998) Carbon and nitrogen isotope discrimination and nitrogen nutrition of trees along a rainfall gradient in northern Australia. Australian Journal of Plant Physiology 25: 413-425.

Schwinning,S. and Ehleringer,J.R. (2001) Water use trade-offs and optimal adaptations to pulse-driven arid ecosystems. Journal of Ecology 89: 464-480.

Shamsutdinov,Z.S. (1988) Ecological-evolutionary principles of selection of arid forage plants. Problems of Desert Development 6: 1-10.

Sharma,A., Dwivedi,B.N., Singh,B., and Kumar,K. (1999) Introduction of Populus euphratica in Indian semi-arid trans Gangetic plains. Annals of Forestry 7: 1-8.

Shmueli,E. (1948) The water balance of some plants of the Dead Sea Salines. Palestine Journal of Botany, Jerusalem Series 4: 117-143.

Singh,K.A. and Thompson,F.B. (1995) Effect of lopping on water potential, transpiration, regrowth, ${ }^{14} \mathrm{C}$-photosynthate distribution and biomass production in Alnus glutinosa. Tree Physiology 15: 197-202.

Smit,G.N. and Rethman,N.F.G. (2000) The influence of tree thinning on the soil water in a semi-arid savanna in southern Africa. J Arid Environ 44: 41-59.

Smith,B.N., Oliver,J., and McMillan,C. (1976) Influence of carbon source, oxygen concentration, light intensity and temperature on ${ }^{13} \mathrm{C} /{ }^{12} \mathrm{C}$ ratios in plant tissues. Botanical Gazette 137: 99-104. 
Sood,R. (2002) Populus euphratica: a new species for fuel-wood. Indian Journal of Forestry 18: 253-254.

Sperry,J.S. and Pockman,W.T. (1993) Limitation of transpiration by hydraulic conductance and xylem cavitation in Betula occidentalis. Plant Cell Environ 17: 1233-1241.

Sperry,J.S., Hacke,U.G., Oren,R., and Comstock,J.P. (2002) Water deficits and hydraulic limits to leaf water supply. Plant Cell Environ 25: 251-263.

Stewart,G.R., Turnbull,M.H., Schmidt,S., and Erskine,P.D. (1995) ${ }^{13} \mathrm{C}$ natural abundance in plant communities along a rainfall gradient: a biological integrator of water availability. Australian Journal of Plant Physiology 22: 51-55.

Terwilliger,V.J., Kitajima,K., Le Roux-Swarthout,D.J., Mulkey,S., and Wright,S.J. (2001) Intrinsic water-use efficiency and heterotrophic investment in tropical leaf growth of two Neotropical tree species as estimated from $\delta^{13} \mathrm{C}$ values. New Phytologist 152: 267-281.

Thomas,D.S. and Eamus,D. (2002) Seasonal patterns of xylem sap pH, xylem abscisic acid concentration, leaf water potential and stomatal conductance of six evergreen and decidous Australian savanna tree species. Australian Journal of Botany 50: 229-236.

Thomas,D.S.G. and Middleton,N.J. (1994) Desertification: Exploding the Myth. Chichester New York Brisbane: John Wiley \& Sons.

Thomas,F.M., Arndt,S.K., Bruelheide,H., Foetzki,A., Gries,D., Huang,J. et al. (2000) Ecological basis for a sustainable management of the indigenous vegetation in a Central-Asian desert: presentation and first results. Journal of Applied Botany 74: 212-219.

Thomas,F.M., Foetzki,A., Gries,D., Arndt,S.K., Bruelheide,H., Li,X. et al. (2002) Plant water relations of four perennials in the perimeter of a Central Asian desert. in Vorbereitung

Tian,C. and Song,Y. (1997) Desertification and its Control in Xinjiang, China. Chinese Journal of Arid Land Research 10: 199-205.

Tian,Y. (1991) Tokai on the delta at the lower reach of the Keriya River - a natural vegetation complex reflecting ecological degradation. Die Erde Suppl.6: 99-112.

Toft,N.L., Anderson,J.E., and Nowak,R.S. (1989) Water use efficiency and carbon isotope composition of plants in a cold desert environment. Oecologia 80: 11-18.

Turner,R.M. (1974) Quantitative and historical evidence of vegetation changes along the Upper Gila River: Arizona. U. S. Geological Survey Prof. Paper 665-H: 20 p.

UNCED . Report of the United Nations Conference on Environment and Development. Vol. I, 1-486. 1992. New York, UN.

UNEP (1992) World Atlas of Desertification. Sevenoaks: Edward Arnold.

van der Walt,J.L. (1953) The camel-thorn bush. Farming in South Africa 30: 401-403.

van Hylckama,T.E.A. (1980) Weather and evapotranspiration studies in a saltcedar thicket, Arizona. Washington D.C.: United States Government Printing Office. 
Voet,D. and Voet,J.G. (1992) Biochemie. Weinheim: VCH.

Walter,H. (1990) Vegetation und Klimazonen. Stuttgart: Ulmer.

Walter,H. and Box,E.O. (1983) The deserts of Central Asia. In Ecosystems of the World 5: Temperate Deserts and Semi-Deserts. West,N.E. (ed). Amsterdam: Elsevier, 193236.

Wanek,W., Heintel,S., and Richter,A. (2001) Preparation of starch and other carbon fractions from higher plant leaves for stable carbon isotope analysis. Rapid Communications in Mass Spectrometry 15: 1136-1140.

Wang,B., Wang,H., and Huang,J. (1997) Responses of photosynthesis and respiration in assimilatory shoots of Calligonum arborescens Litv. to growing season, light intensity, temperature and drought. Scientia Silvae Sinicae 33: 1-24.

Wang,S.J. and Li,H. (1995) Nursery techniques and afforestation of Populus euphratica in China. Pakistan Journal of Forestry 45: 58-64.

Wang,S.J., Chen,B.H., and Li,H.Q. (1996) Euphrates Poplar Forest. Beijing: China Environmental Science Press.

Whittaker,R.H. and Niering,W.A. (1975) Vegetation of the Santa Catalina Mountains, Arizona. V. Biomass, production, and diversity along the elevational gradient. Ecology 56: 771-790.

Wickens,G.E. (1998) Ecophysioloy of Economic Plants in Arid and Semi-Arid Lands. Berlin Heidelberg New York: Springer-Verlag.

Wright,G.C., Hubick,K.T., and Farquhar,G.D. (1988) Discrimination in carbon isotopes of leaves correlates with water-use efficiency of field grown peanuts cultivars.

Australian Journal of Plant Physiology 15: 815-825.

Wu,B. and Ci,L.J. (2002) Landscape change and desertification development in the Mu Us Sandland, Northern China. J Arid Environ 50: 429-444.

Xia,X., Li,C., Zhou,X., Zhang,H., Huang,P., and Pan,B. (1993) Desertification and Control of Blown Sand Disasters in Xinjiang. Beijing: Science Press.

Yang,X.P. (1991) Geomorphologische Untersuchungen in Trockenräumen NW-Chinas unter besonderer Berücksichtigung von Badanjilin und Takelamagan. Göttinger Geographische Abhandlungen 96: -124 p.

Yarashov,A. (1996) Influence of moisture supply on tree stands in the Kara Kum desert. Problems of Desert Development 1: 65-69.

Yoder,C.K. and Nowak,R.S. (1999) Hydraulic lift among native plant species in the Mojave Desert. Plant and Soil 215: 93-102.

Zhang,D.M. and Mao,Z.M. (1990) A study of Calligonum plant communities in Xinjiang. Chinese Journal of Arid Land Research 3: 249-256.

Zhang,H., Gao,S.Y., and Zheng,Q.H. (2002) Responses of NPP of salinized meadows to global change in hyperarid regions. J Arid Environ 50: 489-498. 
Zhao,M., Ge,C., and Zhai,Z. (1997) Study on the determination of salt-tolerance index of the main tree species used for afforestation and their ordination in arid areas with secondary salinization. Forest Research 10: 194-198.

Zheng,D., Liu,J., and Huang,J. (2001) Individual Partner Final Report: Ecomony Institute, Xinjiang Academy of Social Sciences, Urumqi. In Final Project Report - Ecological Basis of a Sustainable Management of the Indigenous Vegetation in a Central Asian Desert. INCO-DC: ERBIC18CT980275. Runge,M. (ed).

Zhu,Z. and Liu,S. (1988) Desertification Processes and Their Control In Northern China. Chinese Journal of Arid Land Research 1: 27-36. 


\section{Anhang}

Tab. 1: Verhältnis von Globalstrahlung und Saftfluss von Alhagi sparsifolia 1999 und 2000.

Angegeben sind die Kurvengleichungen der Beziehung von Sensor 1 (für 1999) bzw. Sensor 2 (S 2) oder Sensor 3 (S 3) für 2000 gegen die Globalstrahlung mit Regressionskoeffizient und Irrtumswahrscheinlichkeit.

\begin{tabular}{c|c|cc|cc|c}
\hline \multicolumn{7}{c}{ Alhagi sparsifolia 1999 } \\
\hline Monat & Gleichung & $\mathbf{a}$ & $\mathbf{P}$ & $\mathbf{b}$ & $\mathbf{P}$ & $\mathbf{r}^{2}$ \\
\hline Mai - Jun & $\mathrm{y}=\mathrm{a}^{*}\left(1-\exp \left(-\mathrm{b}^{*} \mathrm{x}\right)\right)$ & 98.681 & $<0.0001$ & 0.0034 & $<0.0001$ & 0.796 \\
Jun - Jul & $\mathrm{y}=\mathrm{a}^{*}\left(1-\exp \left(-\mathrm{b}^{*} \mathrm{x}\right)\right)$ & 168.663 & $<0.0001$ & 0.0054 & $<0.0001$ & 0.844 \\
Aug - Sep & $\mathrm{y}=\mathrm{a}^{*}\left(1-\exp \left(-\mathrm{b}^{*} \mathrm{x}\right)\right)$ & 179.147 & $<0.0001$ & 0.0067 & $<0.0001$ & 0.778 \\
\hline
\end{tabular}

\begin{tabular}{c|c|ccc|c|c}
\hline \multicolumn{7}{|c}{ Alhagi sparsifolia 2000 } \\
\hline Monat & Gleichung & y0 & a & b & P & $\mathbf{r}^{2}$ \\
\hline Mai (S 2) & $\mathrm{y}=\mathrm{a}^{*} \mathrm{x}+\mathrm{b}$ & & 0.142 & 16.054 & $<0.0001$ & 0.848 \\
Jun (S 3) & $\mathrm{y}=\mathrm{y} 0+\mathrm{a}^{*}\left(1-\exp \left(-\mathrm{b}^{*} \mathrm{x}\right)\right)$ & 20.364 & 134.007 & 0.0049 & $<0.0001$ & 0.884 \\
Jun (S 2) & $\mathrm{y}=\mathrm{a}^{*}\left(1-\exp \left(-\mathrm{b}^{*} \mathrm{x}\right)\right)$ & & 168.473 & 0.0075 & $<0.0001$ & 0.675 \\
Jul (S 3) & $\mathrm{y}=\mathrm{y} 0+\mathrm{a}^{*}\left(1-\exp \left(-\mathrm{b}^{*} \mathrm{x}\right)\right)$ & 24.757 & 147.283 & 0.0049 & $<0.0001$ & 0.935 \\
\hline
\end{tabular}

Tab. 2: Modellierung des Saftflusses von Alhagi sparsifolia 1999 und 2000 Beziehung der Sensoren zueinander.

Angegeben sind die Kurvengleichungen der Regressionen der Sensoren 2 bis 6 gegen Sensor 1 mit Regressionskoeffizient und Irrtumswahrscheinlichkeit. Für verschiedene Zeiträume wurden unterschiedliche Berechnungen verwendet.

\begin{tabular}{c|c|c|c|c|c|c}
\hline \multicolumn{7}{c}{ Alhagi sparsifolia 1999 } \\
\hline Sensor & Monat & Gleichung & $\mathbf{a}$ & $\mathbf{b}$ & $\mathbf{P}$ & $\mathbf{r}^{2}$ \\
\hline 2 vs. 1 & Mai & $\mathrm{y}=\mathrm{a}^{*} \mathrm{x}-\mathrm{b}$ & 1.203 & 6.649 & $<0.0001$ & 0.920 \\
& Jun & $\mathrm{y}=\mathrm{a}^{*} \mathrm{x}+\mathrm{b}$ & 1.351 & 5.997 & $<0.0001$ & 0.996 \\
& Jul - Sep & $\mathrm{y}=\mathrm{a}^{*} \exp \left(\mathrm{b}^{*} \mathrm{x}\right)$ & 26.737 & 0.0148 & $<0.0001$ & 0.982 \\
\hline \multirow{3}{*}{ vs. 1 } & Mai + Jun & $\mathrm{y}=\mathrm{a}^{*} \mathrm{x}-\mathrm{b}$ & 1.795 & 0.706 & $<0.0001$ & 0.982 \\
& Jul + Aug & $\mathrm{y}=\mathrm{a}^{*} \mathrm{x}-\mathrm{b}$ & 1.432 & 3.997 & $<0.0001$ & 0.946 \\
& Sep & $\mathrm{y}=\mathrm{a}^{*} \mathrm{x}-\mathrm{b}$ & 1.457 & 3.373 & $<0.0001$ & 0.935 \\
\hline \multirow{2}{*}{ vs. 1 } & Mai + Jun & $\mathrm{y}=\mathrm{a}^{*} \mathrm{x}+\mathrm{b}$ & 1.539 & 4.282 & $<0.0001$ & 0.996 \\
& Jul - Sep & $\mathrm{y}=\mathrm{a}^{*} \exp \left(\mathrm{b}^{*} \mathrm{x}\right)$ & 25.737 & 0.0129 & $<0.0001$ & 0.991 \\
\hline $\mathbf{5}$ vs. 1 & Mai + Sep & $\mathrm{y}=\mathrm{a}^{*} \mathrm{x}^{\wedge} \mathrm{b}$ & 5.265 & 0.724 & $<0.0001$ & 0.995 \\
& Jun & $\mathrm{y}=\mathrm{a}^{*} \mathrm{x}+\mathrm{b}$ & 0.958 & 13.795 & $<0.0001$ & 0.921 \\
& Jul + Aug & $\mathrm{y}=\mathrm{a}^{*} \mathrm{x}+\mathrm{b}$ & 1.268 & 0.696 & $<0.0001$ & 0.829 \\
\hline $\mathbf{6}$ vs. 1 & Jun & $\mathrm{y}=\mathrm{a}^{*} \mathrm{x}+\mathrm{b}$ & 0.773 & 2.327 & $<0.0001$ & 0.989 \\
& Juli & $\mathrm{y}=\mathrm{a}^{*} \exp \left(\mathrm{b}^{*} \mathrm{x}\right)$ & 11.308 & 0.014 & $<0.0001$ & 0.983 \\
& Aug & $\mathrm{y}=\mathrm{a}^{*} \exp \left(\mathrm{b}^{*} \mathrm{x}\right)$ & 13.519 & 0.0135 & $<0.0001$ & 0.968 \\
& Sep & $\mathrm{y}=\mathrm{a}^{*} \exp \left(\mathrm{b}^{*} \mathrm{x}\right)$ & 14.101 & 0.014 & $<0.0001$ & 0.985 \\
\hline
\end{tabular}




\begin{tabular}{|c|c|c|c|c|c|c|}
\hline \multicolumn{7}{|c|}{ Alhagi sparsifolia 2000} \\
\hline Sensor & Monat & Gleichung & $\mathbf{a}$ & b & $\mathbf{P}$ & $r^{2}$ \\
\hline \multirow[t]{2}{*}{2 vs. 3} & Jun & $y=a^{*} x-b$ & 1.586 & 5.660 & $<0.0001$ & 0.957 \\
\hline & Jul & $y=a+b^{*} x-c^{*} x^{2}$ & -21.73 & $\begin{array}{c}2.42 \\
c=0.0068\end{array}$ & $<0.0001$ & 0.950 \\
\hline 3 vs. 2 & Mai & $y=a^{*} x^{\wedge} b$ & 1.344 & 0.864 & $<0.0001$ & 0.971 \\
\hline 4 vs. 3 & Jul & $\begin{array}{l}y=y 0+a / \\
(1+\exp (-(x-x 0) / b))\end{array}$ & $\begin{array}{c}29.767 \\
\mathrm{y} 0=7.647\end{array}$ & $\begin{array}{c}26.493 \\
x 0=135.144\end{array}$ & $<0.0001$ & 0.732 \\
\hline 5 vs. 2 & Mai & $y=a^{*} x^{\wedge} b$ & 1.263 & 0.517 & $<0.0001$ & 0.711 \\
\hline \multirow[t]{2}{*}{5 vs. 3} & Jun & $\begin{array}{l}y=y 0+a / \\
(1+\exp (-(x-x 0) / b))\end{array}$ & $\begin{array}{c}13.034 \\
v 0=7.137\end{array}$ & $\begin{array}{c}27.492 \\
\times 0=98.86\end{array}$ & $<0.0001$ & 0.861 \\
\hline & Jul & $\begin{array}{l}y=y 0+a / \\
\quad(1+\exp (-(x-x 0) / b))\end{array}$ & $\begin{array}{c}12.415 \\
\text { y0 }=7.432\end{array}$ & $\begin{array}{c}26.993 \\
\times 0=125.037 \\
\end{array}$ & & 0.898 \\
\hline 6 vs. 3 & Jul & $\begin{aligned} y= & y 0+a / \\
& (1+\exp (-(x-x 0) / b))\end{aligned}$ & $\begin{array}{c}37.393 \\
\text { y0 }=11.20\end{array}$ & $\begin{array}{c}26.193 \\
\mathbf{x} 0=107.793\end{array}$ & $<0.0001$ & 0.910 \\
\hline
\end{tabular}

Tab. 3: Verhältnis von Globalstrahlung und Saftfluss von Calligonum caputmedusae 1999 und 2000.

Angegeben sind die Kurvengleichungen der Berechnung von Globalstrahlung gegen Sensor 2 (1999) bzw. Sensor 6 (2000) mit Regressionskoeffizient und Irrtumswahrscheinlichkeit.

\begin{tabular}{|c|c|c|c|c|c|c|}
\hline \multicolumn{7}{|c|}{ Calligonum caput-medusae 1999} \\
\hline Monat & Gleichung & y0 & $\mathbf{a}$ & b & $\mathbf{P}$ & $r^{2}$ \\
\hline Mai & $\mathrm{y}=\mathrm{y} 0+a^{*}\left(1-\exp \left(-b^{*} x\right)\right)$ & 21.957 & 98.034 & 0.0016 & $<0.0001$ & 0.679 \\
\hline Jun + Aug & $y=a^{*}\left(1-\exp \left(-b^{*} x\right)\right)$ & & 99.757 & 0.0019 & $<0.0001$ & 0.791 \\
\hline Jul & $y=a^{*}\left(1-\exp \left(-b^{*} x\right)\right)$ & & 105.110 & 0.0013 & $<0.0001$ & 0.797 \\
\hline Sep & $y=a^{*}\left(1-\exp \left(-b^{*} x\right)\right)$ & & 92.841 & 0.0022 & $<0.0001$ & 0.890 \\
\hline \multicolumn{7}{|c|}{ Calligonum caput-medusae 2000} \\
\hline Monat & Gleichung & y0 & a & b & $\mathbf{P}$ & $r^{2}$ \\
\hline Mai & $y=y 0+a^{*}\left(1-\exp \left(-b^{*} x\right)\right)$ & 11.589 & 78.812 & 0.0019 & $<0.0001$ & 0.876 \\
\hline Jun & $y=y 0+a^{*}\left(1-\exp \left(-b^{*} x\right)\right)$ & 9.089 & 74.966 & 0.0016 & $<0.0001$ & 0.922 \\
\hline
\end{tabular}

Tab. 4: Modellierung des Saftflusses von Calligonum caput-medusae 1999 und 2000.

Angegeben sind die Kurvengleichungen der Regressionen der Sensoren 1 sowie 3 bis 6 gegen Sensor 2 (1999) bzw. Sensor 1 bis 5 gegen Sensor 6 (2000) mit Regressionskoeffizient und Irrtumswahrscheinlichkeit.

\begin{tabular}{|c|c|c|c|c|c|c|c|}
\hline \multicolumn{8}{|c|}{ Calligonum caput-medusae 1999} \\
\hline Sensor & Monat & Gleichung & $a$ & b & $\mathbf{x} \mathbf{0}$ bzw. $\mathbf{c}$ & $\mathbf{P}$ & $r^{2}$ \\
\hline \multirow[t]{5}{*}{1 vs. 2} & Mai & $y=a /(1+\exp (-(x-x 0) / b))$ & 226.63 & 16.396 & 61.719 & $<0.0001$ & 0.959 \\
\hline & Jun & $y=a^{*}\left(1-\exp \left(-b^{*} x\right)\right)$ & 277.790 & 0.0149 & & $<0.0001$ & 0.960 \\
\hline & Jul & $y=a+b^{*} x+c^{*} x^{2}$ & -13.214 & 4.564 & -0.022 & $<0.0001$ & 0.967 \\
\hline & Aug & $y=x 0+a^{*} x^{\wedge} b$ & 13.177 & 0.661 & -23.598 & $<0.0001$ & 0.892 \\
\hline & Sep & $y=x 0+a^{*}\left(1-e x p\left(-b^{*} x\right)\right)$ & 269.969 & 0.0189 & -8.963 & $<0.0001$ & 0.962 \\
\hline 3 vs. 2 & Jul & $y=a^{*} x^{\wedge} b$ & 2.110 & 0.765 & & $<0.0001$ & 0.904 \\
\hline \multirow[t]{3}{*}{4 vs. 2} & Jul & $y=a^{*} x+b$ & 0.695 & -1.051 & & $<0.0001$ & 0.951 \\
\hline & Aug & $y=a^{*}\left(1-\exp \left(-b^{*} x\right)\right)$ & 98.239 & 0.0085 & & $<0.0001$ & 0.962 \\
\hline & Sep & $\mathrm{y}=\mathrm{a}^{*}\left(1-\exp \left(-\mathrm{b}^{*} \mathrm{x}\right)\right)$ & 98.919 & 0.0102 & & $<0.0001$ & 0.975 \\
\hline 5 vs. 2 & Jul & $y=a^{*}\left(1-\exp \left(-b^{*} x\right)\right)$ & 59.876 & 0.025 & & $<0.0001$ & 0.894 \\
\hline \multirow[t]{3}{*}{6 vs. 2} & Mai & $y=a /(1+\exp (-(x-x 0) / b))$ & 173.418 & 23.444 & 65.710 & $<0.0001$ & 0.954 \\
\hline & Jun & $y=x 0+a^{*} x^{\wedge} b$ & 0.417 & 1.253 & 10.613 & $<0.0001$ & 0.873 \\
\hline & Jul & $y=a+b^{*} x+c^{*} x^{2}$ & -0.9344 & 1.414 & -0.00364 & $<0.0001$ & 0.968 \\
\hline
\end{tabular}




\begin{tabular}{c|c|c|ccc|c|c}
\hline \multicolumn{7}{c}{ Calligonum caput-medusae 2000 } \\
\hline Sensor & Monat & Gleichung & $\mathbf{a}$ & $\mathbf{b}$ & $\mathbf{x} \mathbf{0}$ & $\mathbf{P}$ & $\mathbf{r}^{2}$ \\
\hline 1 vs. 6 & Mai & $\mathrm{y}=\mathrm{a} /(1+\exp (-(\mathrm{x}-\mathrm{x} 0) / \mathrm{b}))$ & 159.89 & 20.835 & 55.274 & $<0.0001$ & 0.851 \\
\hline 2 vs. 6 & Mai & $\mathrm{y}=\mathrm{x} 0+\mathrm{a}^{*} \mathrm{x}^{\wedge} \mathrm{b}$ & 0.896 & 0.863 & -2.811 & $<0.0001$ & 0.909 \\
& Jun & $\mathrm{y}=\mathrm{a} /(1+\exp (-(\mathrm{x}-\mathrm{x} 0) / \mathrm{b}))$ & 39.056 & 10.776 & 35.301 & $<0.0001$ & 0.915 \\
\hline 3 vs. 6 & Mai & $\mathrm{y}=\mathrm{a}^{*}\left(1-\exp \left(-\mathrm{b}^{*} \mathrm{x}\right)\right)$ & 29.366 & 0.0196 & & $<0.0001$ & 0.802 \\
& Jun & $\mathrm{y}=\mathrm{a} /(1+\exp (-(\mathrm{x}-\mathrm{x} 0) / \mathrm{b}))$ & 20.905 & 12.663 & 22.692 & $<0.0001$ & 0.725 \\
\hline 5 vs. 6 & Mai & $\mathrm{y}=\mathrm{x} 0+\mathrm{a}^{*}\left(1-\exp \left(-\mathrm{b}^{*} \mathrm{x}\right)\right)$ & 62.833 & 0.0146 & -2.308 & $<0.0001$ & 0.917 \\
& Jun & $\mathrm{y}=\mathrm{a} /(1+\exp (-(\mathrm{x}-\mathrm{x} 0) / \mathrm{b}))$ & 31.898 & 10.434 & 31.083 & $<0.0001$ & 0.923 \\
\hline
\end{tabular}

Tab. 5: Verhältnis von Wasserdampfsättigungsdefizit (VPD) und Saftfluss von Populus euphratica 1999 und 2000.

Angegeben sind die Kurvengleichungen der Berechnung von Sensor 3 (1999) bzw. Sensor 4 (2000) gegen das VPD mit Regressionskoeffizient und Irrtumswahrscheinlichkeit.

\begin{tabular}{c|c|cc|c|c}
\hline \multicolumn{7}{c}{ Populus euphratica 1999 } \\
\hline Monat & Gleichung & $\mathbf{a}$ & $\mathbf{b}$ & $\mathbf{P}$ & $\mathrm{r}^{2}$ \\
\hline Mai & $\mathrm{y}=\mathrm{a}^{*} \mathrm{x}+\mathrm{b}$ & 0.018 & 0.434 & $<0.0001$ & 0.620 \\
Jun & $\mathrm{y}=\mathrm{a}^{*} \mathrm{x}+\mathrm{b}$ & -0.0228 & 0.3917 & 0.001 & 0.525 \\
& $\mathrm{y}=\mathrm{a}^{*} \mathrm{x}+\mathrm{b}$ & -0.0695 & 0.411 & $<0.0001$ & 0.613 \\
Sep (S3) & $\mathrm{y}=\mathrm{a}^{*} \mathrm{x}+\mathrm{b}$ & -0.0232 & 0.3562 & $<0.0001$ & 0.343 \\
Sep (S1) & $\mathrm{y}=\mathrm{a}^{*} \mathrm{x}+\mathrm{b}$ & -0.02 & 0.3581 & $<0.0001$ & 0.31 \\
\hline
\end{tabular}

\begin{tabular}{c|c|cc|c|c}
\hline \multicolumn{7}{|c}{ Populus euphratica 2000 } \\
\hline Monat & Gleichung & $\mathbf{a}$ & $\mathbf{b}$ & $\mathbf{P}$ & $\mathrm{r}^{2}$ \\
\hline Mai & $\mathrm{y}=\mathrm{a}^{*} \mathrm{x}+\mathrm{b}$ & 0.0315 & 0.4918 & 0.0002 & 0.631 \\
& $\mathrm{y}=\mathrm{a}^{*} \mathrm{x}+\mathrm{b}$ & -0.0163 & 0.4937 & $<0.0001$ & 0.580 \\
\hline Mai-Jun & $\mathrm{y}=\mathrm{a}^{*} \mathrm{x}+\mathrm{b}$ & -0.0225 & 0.4551 & 0.0006 & 0.642 \\
Jun & $\mathrm{y}=\mathrm{a}^{*} \mathrm{x}+\mathrm{b}$ & -0.0153 & 0.4291 & $<0.0001$ & 0.811 \\
& $\mathrm{y}=\mathrm{a}^{*} \mathrm{x}+\mathrm{b}$ & -0.0167 & 0.4344 & $<0.0001$ & 0.516 \\
\hline Jul & $\mathrm{y}=\mathrm{a}^{*} \mathrm{x}+\mathrm{b}$ & -0.087 & 0.4237 & $<0.0001$ & 0.830 \\
& $\mathrm{y}=\mathrm{a}^{*} \mathrm{x}+\mathrm{b}$ & 0.0311 & 0.5054 & $<0.0001$ & 0.601 \\
\hline Aug & $\mathrm{y}=\mathrm{a}^{*} \mathrm{x}+\mathrm{b}$ & -0.0455 & 0.5166 & $<0.0001$ & 0.867 \\
Aug-Sep & $\mathrm{y}=\mathrm{a}^{*} \mathrm{x}+\mathrm{b}$ & -0.0385 & 0.518 & $<0.0001$ & 0.713 \\
\hline
\end{tabular}

Tab. 6: Modellierung des Saftflusses von Populus euphratica 1999 und 2000.

Angegeben sind die Kurvengleichungen der Regressionen der Sensoren 2 und 3 vs. Sensor 4 (August 1999) bzw. Sensor 4 und 5 vs. Sensor 3 (September 1999) und Sensor 5 vs. Sensor 4 (2000) mit Regressionskoeffizient und Irrtumswahrscheinlichkeit.

\begin{tabular}{|c|c|c|c|c|c|c|}
\hline \multicolumn{7}{|c|}{ Populus euphratica 1999} \\
\hline Sensor & Monat & Gleichung & a & b & $\mathbf{P}$ & $r^{2}$ \\
\hline 2 vs. 4 & Aug + Sep & $y=a^{*} x+b$ & 0.6603 & 0.1623 & $<0.0001$ & 0.614 \\
\hline 3 vs. 4 & Aug & $y=a^{*} x+b$ & 1.0129 & 0.664 & $<0.0001$ & 0.683 \\
\hline 4 vs. 3 & Jul - Sep & $y=a * x+b$ & 0.6977 & 0.1404 & $<0.0001$ & 0.708 \\
\hline 5 vs. 3 & Aug + Sep & $y=a^{*} x+b$ & 0.894 & 0.0097 & $<0.0001$ & 0.856 \\
\hline \multicolumn{7}{|c|}{ Populus euphratica 2000} \\
\hline Sensor & Monat & Gleichung & a & b & $\mathbf{P}$ & $r^{2}$ \\
\hline 5 vs. 4 & Mai & $y=a^{*} x-b$ & 0.81 & 0.0425 & $<0.0001$ & 0.613 \\
\hline 5 vs. 4 & Jun & $y=a^{*} x+b$ & 0.856 & & $<0.0001$ & 0.616 \\
\hline 5 vs. 4 & Jul & $y=a^{*} x+b$ & 0.4623 & 0.1351 & $<0.0001$ & 0.368 \\
\hline 5 vs. 4 & Aug + Sep & $y=a * x-b$ & 0.801 & 0.0336 & $<0.0001$ & 0.683 \\
\hline
\end{tabular}


Tab. 7: Verhältnis von Globalstrahlung und Saftfluss von Tamarix ramosissima 1999 und 2000.

Angegeben sind die Kurvengleichungen der Berechnung von Globalstrahlung gegen Sensor 1 (1999) bzw. Sensor 6 (2000) mit Regressionskoeffizient und Irrtumswahrscheinlichkeit.

\section{Tamarix ramosissima 1999}

\begin{tabular}{c|c|ccc|c|c}
\hline Monat & Gleichung & $\mathbf{a}$ & $\mathbf{b}$ & $\mathbf{y}$ 0 bzw. C & $\mathbf{P}$ & $\mathbf{r}^{2}$ \\
\hline Mai & $\mathrm{y}={\mathrm{a}+\mathrm{b}^{*} \mathrm{x}-\mathrm{c}^{*} \mathrm{x}^{2}}^{*}$ & 4.644 & 0.207 & -0.000113 & $<0.0001$ & 0.962 \\
Mai/Jun & $\mathrm{y}=\mathrm{y} 0+\mathrm{a}^{*}\left(1-\exp \left(-\mathrm{b}^{*} \mathrm{x}\right)\right)$ & 125.757 & 0.0021 & 3.073 & $<0.0001$ & 0.923 \\
Jun & $\mathrm{y}=\mathrm{y} 0+\mathrm{a}^{*}\left(1-\exp \left(-\mathrm{b}^{*} \mathrm{x}\right)\right)$ & 195.134 & 0.0016 & 3.785 & $<0.0001$ & 0.949 \\
Jul & $\mathrm{y}=\mathrm{y} 0+\mathrm{a}^{*}\left(1-\exp \left(-\mathrm{b}^{*} \mathrm{x}\right)\right)$ & 226.176 & 0.0013 & 4.177 & $<0.0001$ & 0.935 \\
Aug & $\mathrm{y}=\mathrm{a}+\mathrm{b}^{*} \mathrm{x}-\mathrm{c}^{*} \mathrm{x}^{2}$ & 3.679 & 0.2360 & -0.0000644 & $<0.0001$ & 0.932 \\
Sep & $\mathrm{y}=\mathrm{a}+\mathrm{b}^{*} \mathrm{x}-\mathrm{c}^{*} \mathrm{x}^{2}$ & 5.456 & 0.209 & -0.0000383 & $<0.0001$ & 0.959 \\
\hline
\end{tabular}

\section{Tamarix ramosissima 2000}

\begin{tabular}{c|c|ccc|c|c}
\hline Monat & Gleichung & a & b & y0 & P & $\mathbf{r}^{2}$ \\
\hline Mai & $\mathrm{y}=\mathrm{y} 0+\mathrm{a}^{*}\left(1-\exp \left(-\mathrm{b}^{*} \mathrm{x}\right)\right)$ & 79.180 & 0.0043 & 21.465 & $<0.0001$ & 0.834 \\
\hline
\end{tabular}

Tab. 8: Modellierung des Saftflusses von Tamarix ramosissima 1999 und 2000.

Angegeben sind die Kurvengleichungen der Regressionen der Sensoren 2 bis 6 gegen Sensor 1 (1999) bzw. Sensor 1 bis 5 gegen Sensor 6 (2000) mit Regressionskoeffizient und Irrtumswahrscheinlichkeit.

\begin{tabular}{|c|c|c|c|c|c|c|c|}
\hline \multicolumn{8}{|c|}{ Tamarix ramosissima 1999} \\
\hline Sensor & Monat & Gleichung & a & b & xO bzw. $\mathbf{c}$ & $\mathbf{P}$ & $r^{2}$ \\
\hline \multirow[t]{3}{*}{2 vs. 1} & Jun & $y=a^{*}\left(1-\exp \left(-b^{*} x\right)\right)$ & 79.718 & 0.0262 & \multirow{3}{*}{-0.103} & $<0.0001$ & 0.968 \\
\hline & Aug & $y=x 0+a^{*}\left(1-e x p\left(-b^{*} x\right)\right)$ & 84.965 & 0.0283 & & $<0.0001$ & 0.969 \\
\hline & Sep & $y=a^{*}\left(1-\exp \left(-b^{*} x\right)\right)$ & 81.091 & 0.0247 & & $<0.0001$ & 0.962 \\
\hline \multirow[t]{4}{*}{3 vs. 1} & Jun & $y=a^{*} x^{\wedge} b$ & 7.223 & 0.652 & \multirow{4}{*}{$\begin{array}{r}2.304 \\
-2.132 \\
-4.389 \\
\end{array}$} & $<0.0001$ & 0.982 \\
\hline & Jul & $y=x 0+a^{*}\left(1-\exp \left(-b^{*} x\right)\right)$ & 223.304 & 0.0109 & & $<0.0001$ & 0.986 \\
\hline & Aug & $y=x 0+a^{*}\left(1-\exp \left(-b^{*} x\right)\right)$ & 218.610 & 0.0134 & & $<0.0001$ & 0.984 \\
\hline & Sep & $y=x 0+a^{*}\left(1-\exp \left(-b^{*} x\right)\right)$ & 228.575 & 0.0138 & & $<0.0001$ & 0.983 \\
\hline \multirow[t]{4}{*}{4 vs. 1} & Jun & $y=a^{*}\left(1-\exp \left(-b^{*} x\right)\right)$ & 275.352 & 0.0098 & \multirow{4}{*}{$\begin{array}{l}-2.146 \\
-5.514\end{array}$} & $<0.0001$ & 0.969 \\
\hline & Jul & $y=x 0+a^{*}\left(1-\exp \left(-b^{*} x\right)\right)$ & 241.701 & 0.0131 & & $<0.0001$ & 0.972 \\
\hline & Aug & $y=x 0+a^{*}\left(1-\exp \left(-b^{*} x\right)\right)$ & 231.334 & 0.0168 & & $<0.0001$ & 0.968 \\
\hline & Sep & $y=a^{*}\left(1-\exp \left(-b^{*} x\right)\right)$ & 240.380 & 0.0114 & & $<0.0001$ & 0.953 \\
\hline \multirow[t]{6}{*}{5 vs. 1} & Mai & $y=x 0+a^{*} x^{\wedge} b$ & 9.939 & 0.656 & \multirow[t]{6}{*}{-4.820} & $<0.0001$ & 0.951 \\
\hline & Jun & $y=a^{*} x^{\wedge} b$ & 8.337 & 0.707 & & $<0.0001$ & 0.943 \\
\hline & Jul & (Anfang Juli) $y=a^{*} x+b$ & 3.123 & 10.636 & & $<0.0001$ & 0.933 \\
\hline & & (Ende Juli) $y=a^{*} x+b$ & 0.252 & 7.701 & & $<0.0001$ & 0.785 \\
\hline & Aug & $y=a+b^{*} x+c^{*} x^{2}$ & 2.508 & 1.0083 & & $<0.0001$ & 0.899 \\
\hline & Sep & $y=a^{*}\left(1-\exp \left(-b^{*} x\right)\right)$ & 161.663 & 0.0122 & & $<0.0001$ & 0.930 \\
\hline \multirow[t]{2}{*}{6 vs. 1} & Jun + Jul & $y=a^{*}\left(1-\exp \left(-b^{*} x\right)\right)$ & 123.935 & 0.0288 & \multirow{3}{*}{$\begin{array}{c}4.054 \\
-0.00736\end{array}$} & $<0.0001$ & 0.957 \\
\hline & Aug & $y=a+b^{*} x+c^{*} x^{2}$ & 13.289 & 1.477 & & $<0.0001$ & 0.855 \\
\hline 6 vs. 2 & Sep & $y=a^{*}\left(1-\exp \left(-b^{*} x\right)\right)$ & 79.420 & 0.038 & & $<0.0001$ & 0.895 \\
\hline
\end{tabular}

\section{Tamarix ramosissima 2000}

\begin{tabular}{c|c|c|ccc|c|c}
\hline Sensor & Monat & Gleichung & $\mathbf{a}$ & $\mathbf{b}$ & $\mathbf{x} \mathbf{0}$ & $\mathbf{P}$ & $\mathbf{r}^{2}$ \\
\hline 1 vs. 6 & Mai & $\mathrm{y}=\mathrm{a} /(1+\exp (-(\mathrm{x}-\mathrm{x} 0) / \mathrm{b}))$ & 159.89 & 20.835 & 55.274 & $<0.0001$ & 0.851 \\
\hline 2 vs. 6 & Mai & $\mathrm{y}=\mathrm{x} 0+\mathrm{a}^{*} \mathrm{x}^{\wedge} \mathrm{b}$ & 0.896 & 0.863 & -2.811 & $<0.0001$ & 0.909 \\
& Jun & $\mathrm{y}=\mathrm{a} /(1+\exp (-(\mathrm{x}-\mathrm{x} 0) / \mathrm{b}))$ & 39.056 & 10.776 & 35.301 & $<0.0001$ & 0.915 \\
\hline \multirow{2}{*}{ vs. 6 } & Mai & $\mathrm{y}=\mathrm{a}^{*}\left(1-\exp \left(-\mathrm{b}^{*} \mathrm{x}\right)\right)$ & 29.366 & 0.0196 & & $<0.0001$ & 0.802 \\
& Jun & $\mathrm{y}=\mathrm{a} /(1+\exp (-(\mathrm{x}-\mathrm{x} 0) / \mathrm{b}))$ & 20.905 & 12.663 & 22.692 & $<0.0001$ & 0.725 \\
\hline 5 vs. 6 & Mai & $\mathrm{y}=\mathrm{x} 0+\mathrm{a}^{*}\left(1-\exp \left(-\mathrm{b}^{*} \mathrm{x}\right)\right)$ & 62.833 & 0.0146 & -2.308 & $<0.0001$ & 0.917 \\
& Jun & $\mathrm{y}=\mathrm{a} /(1+\exp (-(\mathrm{x}-\mathrm{x} 0) / \mathrm{b}))$ & 31.898 & 10.434 & 31.083 & $<0.0001$ & 0.923 \\
\hline
\end{tabular}


Tab. 9: Wasserpotentiale von der vier untersuchten Arten 1999

Angegeben sind die Mittelwerte der Wasserpotentiale $(\Psi)$ in MPa von 18 Messungen 6 Pflanzen mit je 3 Parallelen) mit Standardfehler (se). Gemessen wurde auf den bewässerten ungenutzten Flächen (FN).

\begin{tabular}{|c|c|c|c|c|c|c|c|c|c|c|c|}
\hline \multicolumn{12}{|c|}{ Alhagi sparsifolia 1999} \\
\hline \multicolumn{3}{|c|}{ 15.05.1999 } & \multicolumn{3}{|c|}{09.07 .1999} & \multicolumn{3}{|c|}{16.08 .1999} & \multicolumn{3}{|c|}{ 25.09.1999 } \\
\hline Zeit & $\Psi$ & se & Zeit & $\Psi$ & se & Zeit & $\Psi$ & se & Zeit & $\Psi$ & se \\
\hline $7: 20$ & -0.239 & 0.018 & 7:37 & -0.565 & 0.033 & 7:02 & -0.393 & 0.017 & $7: 50$ & -0.671 & 0.021 \\
\hline 8:22 & -0.331 & 0.03 & $8: 22$ & -0.556 & 0.0 & $8: 00$ & -0.383 & & :45 & -0.594 & 16 \\
\hline $10: 20$ & -0.246 & 0.026 & 11:05 & -1.803 & 0.016 & $8: 46$ & -0.358 & 0.009 & $10: 18$ & -0.962 & 0.043 \\
\hline $11: 23$ & -1.245 & 0.102 & $12: 22$ & -1.794 & 0.0 & $10: 24$ & -1.059 & 0.0 & $11: 24$ & -1.723 & 0.064 \\
\hline $12: 20$ & -1.559 & 0.046 & & -1.860 & 0.0 & $11: 18$ & -1.235 & & 12:17 & & 0.040 \\
\hline $14: 22$ & -1.518 & 0.02 & & -1.692 & 0.0 & $12: 18$ & -1.459 & & $13: 16$ & -2.091 & 0.033 \\
\hline 15:21 & -1.490 & 0.055 & $16: 44$ & -1.711 & 0.033 & 13:16 & -1.619 & 0.054 & $15: 19$ & -2.101 & 0.029 \\
\hline $16: 23$ & -1.533 & 0.026 & 18:00 & -1.692 & 0.031 & 15:17 & -1.674 & 0.031 & $16: 16$ & -1.985 & 0.026 \\
\hline $17: 20$ & -1.435 & 0.051 & 18:45 & -1.603 & 0.014 & $16: 24$ & -1.789 & & 17 & -2.019 & 0.032 \\
\hline $18: 22$ & -1.425 & 0.059 & & & & $17: 19$ & -1.725 & 0.037 & $18: 19$ & -1.838 & 0.033 \\
\hline $19: 20$ & -1.505 & 0.039 & & & & 18:19 & -1.634 & 0.042 & 19:17 & -1.199 & 0.027 \\
\hline & & & & & & 19:18 & -1.382 & 0.051 & & & \\
\hline
\end{tabular}

\begin{tabular}{|c|c|c|c|c|c|c|c|c|c|c|c|}
\hline \multicolumn{12}{|c|}{ Calligonum caput-medusae 1999} \\
\hline \multicolumn{3}{|c|}{10.05 .1999} & \multicolumn{3}{|c|}{07.06 .1999} & \multicolumn{3}{|c|}{05.07 .1999} & \multicolumn{3}{|c|}{11.08 .1999} \\
\hline Zeit & $\Psi$ & se & Zeit & $\Psi$ & se & Zeit & $\Psi$ & se & Zeit & $\Psi$ & se \\
\hline $7: 37$ & -0.621 & 0.024 & $7: 20$ & -0.584 & 0.014 & $7: 36$ & -0.714 & 0.021 & $7: 07$ & -0.702 & 0.024 \\
\hline 10:15 & -1.055 & 0.025 & $8: 20$ & -0.589 & 0.014 & $8: 47$ & -0.717 & 0.014 & $8: 06$ & -0.607 & 0.011 \\
\hline $11: 25$ & -1.093 & 0.025 & $10: 20$ & -1.199 & 0.034 & $10: 39$ & -1.119 & 0.023 & $10: 47$ & -0.950 & 0.019 \\
\hline $12: 42$ & -1.257 & 0.028 & $11: 20$ & -1.357 & 0.026 & $12: 05$ & -1.433 & 0.032 & $11: 48$ & -1.243 & 0.031 \\
\hline $14: 52$ & -1.456 & 0.037 & $12: 20$ & -1.450 & 0.036 & $13: 37$ & -1.603 & 0.028 & $12: 48$ & -1.314 & 0.035 \\
\hline $16: 22$ & -1.487 & 0.036 & $14: 20$ & -1.6 & & $15: 50$ & -1.626 & 0.035 & & & 0.040 \\
\hline $17: 25$ & -1.366 & 0.031 & $15: 20$ & -1.602 & 0.032 & $17: 25$ & -1.612 & 0.023 & $15: 51$ & -1.450 & 0.035 \\
\hline $18: 23$ & -1.191 & 0.037 & $16: 20$ & -1.573 & 0.039 & $19: 00$ & -1.395 & 0.031 & $16: 52$ & -1.471 & 0.030 \\
\hline \multirow[t]{3}{*}{ 19:20 } & -1.108 & 0.045 & $17: 20$ & -1.539 & 0.036 & & & & $17: 51$ & -1.320 & 0.050 \\
\hline & & & $18: 22$ & -1.500 & 0.054 & & & & $18: 48$ & -1.062 & 0.023 \\
\hline & & & $19: 20$ & -1.314 & 0.044 & & & & $19: 44$ & -0.889 & 0.021 \\
\hline
\end{tabular}

\begin{tabular}{|c|c|c|c|c|c|c|c|c|c|c|c|}
\hline \multirow{2}{*}{\multicolumn{3}{|c|}{$\begin{array}{c}\text { Calligonum } \\
23.09 .1999\end{array}$}} & \multicolumn{9}{|c|}{ Tamarix ramosissima 1999} \\
\hline & & & \multicolumn{3}{|c|}{12.05 .1999} & \multicolumn{3}{|c|}{11.06 .1999} & \multicolumn{3}{|c|}{12.07 .1999} \\
\hline Zeit & $\Psi$ & se & Zeit & $\Psi$ & se & Zeit & $\Psi$ & se & Zeit & $\Psi$ & se \\
\hline $7: 48$ & -0.684 & 0.020 & $7: 17$ & -0.734 & 0.025 & $7: 17$ & -0.879 & 0.052 & $7: 43$ & -1.042 & 0.037 \\
\hline $8: 35$ & -0.668 & 0.012 & $8: 17$ & -0.746 & 0.027 & 9:05 & -0.739 & 0.0 & $8: 27$ & -1.144 & 0.026 \\
\hline $10: 15$ & & 0.0 & & & 0.0 & $10: 35$ & & & $10: 20$ & -1.224 & 37 \\
\hline $11: 17$ & $-1.3 s$ & 0.0 & $10: 25$ & -1.846 & 0.053 & $11: 2$ & & & $11: 15$ & -1.433 & 0.0 \\
\hline $12: 21$ & -1.637 & 0.03 & $11: 30$ & -2.088 & 0.048 & $12: 51$ & -2.042 & 0.1 & $12: 10$ & -1.559 & 0.046 \\
\hline $13: 15$ & & & $12: 27$ & -2.105 & 0.050 & $15: 32$ & -1.874 & 0.113 & $12: 45$ & -1.948 & 0.063 \\
\hline $15: 16$ & & & & -2.2 & 0.034 & & & & $13: 30$ & -2.024 & 0.075 \\
\hline $16: 19$ & -1.717 & 0.0 & $15: 27$ & -2.091 & 0.042 & & & & $15: 50$ & -2.118 & 0.085 \\
\hline $17: 23$ & -1.652 & 0.05 & $16: 28$ & -2.194 & 0.035 & & & & $16: 52$ & -2.064 & 0.082 \\
\hline $18: 18$ & -1.56 & 0.0 & $17: 25$ & -2.112 & 0.033 & & & & & & \\
\hline 19:20 & -1.269 & 0.036 & $18: 22$ & -1.953 & 0.050 & & & & & & \\
\hline & & & $19: 20$ & -1.644 & 0.035 & & & & & & \\
\hline
\end{tabular}




\begin{tabular}{|c|c|c|c|c|c|c|c|c|c|c|c|}
\hline \multicolumn{6}{|c|}{ Tamarix ramosissima 1999} & \multicolumn{6}{|c|}{ Populus euphratica 1999} \\
\hline \multicolumn{3}{|c|}{ 21.08.1999 } & \multicolumn{3}{|c|}{27.09 .1999} & \multicolumn{3}{|c|}{24.05 .1999} & \multicolumn{3}{|c|}{08.06 .1999} \\
\hline Zeit & $\Psi$ & se & Zeit & $\Psi$ & se & Zeit & $\Psi$ & se & Zeit & $\Psi$ & se \\
\hline $7: 22$ & -0.926 & 0.037 & $7: 57$ & -0.967 & 0.045 & $7: 20$ & -0.518 & 0.034 & $7: 30$ & -0.558 & 0.010 \\
\hline $8: 27$ & -0.890 & 0.032 & $8: 51$ & -0.976 & 0.037 & $8: 20$ & -0.713 & 0.021 & 9:00 & -0.950 & 0.043 \\
\hline $10: 25$ & -2.103 & 0.111 & 10:16 & -1.521 & 0.0 & $10: 25$ & -1 & & $10: 25$ & -1.603 & 0.025 \\
\hline $11: 27$ & -2.485 & 0.064 & $11: 15$ & -2.013 & 0.039 & $11: 25$ & -1.394 & 0.028 & $11: 55$ & -1.897 & 0.024 \\
\hline $12: 37$ & -2.830 & 0.069 & $12: 20$ & -2.317 & 0.094 & $12: 25$ & -1.471 & 0.025 & $13: 25$ & -2.001 & 0.014 \\
\hline $15: 32$ & -3.024 & 0.048 & $13: 19$ & -2.749 & 0.071 & $14: 27$ & -1.556 & 0.040 & $15: 27$ & -1.846 & 0.044 \\
\hline $16: 48$ & -2.550 & 0.0 & $15: 16$ & -2.752 & 0.0 & 16: & & & $16: 30$ & -1. & 0.018 \\
\hline $17: 46$ & -2.580 & 0.081 & $16: 18$ & -2.568 & 0.074 & $17: 05$ & -1.315 & 0.037 & $18: 00$ & -1.685 & 0.034 \\
\hline \multirow[t]{3}{*}{ 19:03 } & -2.056 & 0.063 & $17: 16$ & -2.421 & 0.065 & 18:03 & -1.191 & 0.029 & $19: 25$ & -1.590 & 0.051 \\
\hline & & & 18:19 & -2.094 & 0.053 & 19:03 & -1.136 & 0.030 & & & \\
\hline & & & $19: 12$ & -1.726 & 0.037 & & & & & & \\
\hline
\end{tabular}

\begin{tabular}{|c|c|c|c|c|c|c|c|c|c|}
\hline \multicolumn{10}{|c|}{ Populus euphratica 1999} \\
\hline \multicolumn{3}{|c|}{ 08.07.1999 } & \multicolumn{3}{|c|}{18.08 .1999} & \multicolumn{3}{|c|}{14.09 .1999} & \\
\hline Zeit & $\Psi$ & se & Zeit & $\Psi$ & se & Zeit & $\Psi$ & se & \\
\hline $7: 45$ & -0.797 & 0.018 & $7: 36$ & -0.723 & 0.017 & $8: 05$ & -0.712 & 0.024 & \\
\hline $8: 45$ & -1.098 & 0.051 & $8: 14$ & -0.693 & 0.021 & 9:00 & -0.791 & 0.021 & \\
\hline $10: 30$ & -2.002 & 0.035 & $9: 17$ & -0.925 & 0.031 & $10: 18$ & -1.660 & 0.048 & \\
\hline $11: 55$ & -2.176 & 0.029 & $10: 24$ & -1.606 & 0.050 & $11: 21$ & -1.941 & 0.052 & \\
\hline $13: 33$ & -2.324 & 0.022 & $11: 21$ & -1.918 & 0.035 & $12: 34$ & -2.152 & 0.038 & \\
\hline 16:05 & -2.237 & 0.047 & $12: 27$ & -2.177 & 0.039 & $13: 40$ & -2.186 & 0.038 & \\
\hline $17: 30$ & -2.254 & 0.035 & $13: 21$ & -2.307 & 0.023 & $15: 32$ & -2.083 & 0.047 & \\
\hline \multirow{5}{*}{$19: 28$} & -1.975 & 0.047 & $15: 22$ & -2.356 & 0.032 & $16: 35$ & -2.138 & 0.025 & \\
\hline & & & $16: 21$ & -2.206 & 0.036 & $17: 27$ & -2.079 & 0.047 & \\
\hline & & & $17: 26$ & -2.092 & 0.039 & 19:00 & -1.874 & 0.046 & \\
\hline & & & $18: 22$ & -2.040 & 0.037 & & & & \\
\hline & & & $19: 23$ & -1.751 & 0.039 & & & & \\
\hline
\end{tabular}


Tab. 10: Wasserpotentiale 2000

Angegeben sind die Mittelwerte der pre dawn und midday Wasserpotentiale ( $\Psi_{\mathrm{pd}}$ und $\left.\Psi_{\mathrm{md}}\right)$ in MPa von 12 Messungen (4 Pflanzen mit je 3 Parallelen) mit Standardfehler (se).

\begin{tabular}{c|cc|cc}
\hline \multicolumn{5}{c}{ Alhagi sparsifolia AFN } \\
\hline Datum & $\Psi_{\text {pd }}$ & se & $\Psi_{\text {md }}$ & se \\
\hline 22.05 .00 & -0.417 & 0.014 & -1.514 & 0.100 \\
25.06 .00 & -0.592 & 0.017 & -1.888 & 0.048 \\
04.08 .00 & -0.508 & 0.018 & -1.900 & 0.034 \\
16.09 .00 & -0.529 & 0.017 & -2.288 & 0.045 \\
\hline
\end{tabular}

\begin{tabular}{c|cc|cc|c|cc|cc}
\hline \multicolumn{4}{c|}{ Calligonum caput-medusae CFN } & \multicolumn{5}{c}{ Calligonum caput-medusae CFU } \\
\hline Datum & $\Psi_{\text {pd }}$ & se & $\Psi_{\text {md }}$ & se & Datum & $\Psi_{\text {pd }}$ & se & $\Psi_{\text {md }}$ & se \\
\hline 17.05 .00 & -0.568 & 0.021 & -1.565 & 0.066 & 17.05 .00 & -0.668 & 0.022 & -1.699 & 0.030 \\
20.06 .00 & -0.788 & 0.034 & -1.700 & 0.048 & 20.06 .00 & -0.782 & 0.023 & -1.689 & 0.029 \\
$\mathbf{0 3 . 0 8 . 0 0}$ & -0.592 & 0.019 & -1.550 & 0.068 & 03.08 .00 & -0.704 & 0.023 & -1.646 & 0.033 \\
12.09 .00 & -0.779 & 0.018 & -1.663 & 0.043 & 12.09 .00 & -0.658 & 0.026 & -1.667 & 0.033 \\
\hline
\end{tabular}

\begin{tabular}{c|cc|cc|c|cc|cc}
\hline \multicolumn{4}{c|}{ Tamarix ramosissima TFN } & \multicolumn{4}{c}{ Tamarix ramosissima TFU } \\
\hline Datum & $\Psi_{\mathrm{pd}}$ & se & $\Psi_{\mathrm{md}}$ & se & Datum & $\Psi_{\mathrm{pd}}$ & se & $\Psi_{\mathrm{md}}$ & se \\
\hline 18.05 .00 & $-\mathbf{0 . 4 8 7}$ & 0.016 & $-\mathbf{2 . 3 5 8}$ & 0.093 & 18.05 .00 & -0.600 & 0.053 & $\mathbf{- 2 . 4 9 9}$ & 0.041 \\
19.09 .00 & $-\mathbf{0 . 9 5 8}$ & 0.039 & $\mathbf{- 2 . 9 0 8}$ & 0.081 & $\mathbf{1 9 . 0 9 . 0 0}$ & -0.811 & 0.015 & $\mathbf{- 2 . 6 2 9}$ & 0.095 \\
\hline
\end{tabular}

\begin{tabular}{c|cc|cc|c|cc|cc}
\hline \multicolumn{4}{c|}{ Populus euphratica PFN } & \multicolumn{4}{c}{ Populus euphratica PFU } \\
\hline Datum & $\Psi_{\mathrm{pd}}$ & se & $\Psi_{\mathrm{md}}$ & se & Datum & $\Psi_{\mathrm{pd}}$ & se & $\Psi_{\mathrm{md}}$ & se \\
\hline $\mathbf{2 9 . 0 5 . 0 0}$ & $-\mathbf{0 . 5 6 0}$ & 0.015 & -1.895 & 0.034 & $\mathbf{2 9 . 0 5 . 0 0}$ & $-\mathbf{0 . 5 2 8}$ & 0.005 & $\mathbf{- 1 . 6 5 3}$ & 0.039 \\
$\mathbf{0 4 . 0 7 . 0 0}$ & $-\mathbf{0 . 9 1 7}$ & 0.022 & $\mathbf{- 2 . 0 2 5}$ & 0.058 & $\mathbf{0 4 . 0 7 . 0 0}$ & $\mathbf{- 0 . 8 2 9}$ & 0.021 & $\mathbf{- 1 . 8 6 3}$ & 0.061 \\
$\mathbf{1 2 . 0 8 . 0 0}$ & $-\mathbf{0 . 7 1 3}$ & 0.012 & $\mathbf{- 2 . 3 7 9}$ & 0.030 & $\mathbf{1 2 . 0 8 . 0 0}$ & $\mathbf{- 0 . 7 7 9}$ & 0.022 & $\mathbf{- 2 . 3 4 6}$ & 0.048 \\
$\mathbf{2 5 . 0 9 . 0 0}$ & $-\mathbf{0 . 7 5 8}$ & 0.016 & $\mathbf{- 2 . 3 5 4}$ & 0.055 & $\mathbf{2 5 . 0 9 . 0 0}$ & $-\mathbf{0 . 6 6 0}$ & 0.006 & $\mathbf{- 1 . 8 5 7}$ & 0.024 \\
\hline
\end{tabular}

Tab. 11: Vergleich der mikroklimatischen Bedingungen und der Saftflusssumme vor und nach der Bewässerung 1999.

Angegeben sind die Mittelwerte des trockengewichtsbezogenen Saftflusses von allen Sensoren an mikroklimatisch ähnlichen Tagen kurz vor und kurz nach der Bewässerung. Es wurden jeweils die Werte der nächstgelegenen Klimastation verwendet.

\begin{tabular}{|c|c|c|c|c|c|c|c|}
\hline Datum & $\begin{array}{c}\text { RH min } \\
{[\%]}\end{array}$ & $\begin{array}{c}\text { RH max } \\
{[\%]}\end{array}$ & $\begin{array}{c}\text { Tagesmittel- } \\
\text { temperatur } \\
{\left[{ }^{\circ} \mathrm{C}\right]} \\
\end{array}$ & $\begin{array}{c}\text { Strahlungs- } \\
\text { summe } \\
{\left[\mathrm{W}^{*} \mathrm{~m}^{-2}\right]} \\
\end{array}$ & $\begin{array}{l}\text { Summe PAR } \\
{\left[\mu \mathrm{mol}^{*} \mathrm{~m}^{-2 *} \mathrm{~s}^{-1}\right]}\end{array}$ & $\begin{array}{c}\text { Mittleres } \\
\text { VPD } \\
{[\mathrm{kPa}]} \\
\end{array}$ & $\begin{array}{c}\text { Saftfluss- } \\
\text { summe } \\
{\left[g^{*} \mathrm{Tag}^{-1}\right]}\end{array}$ \\
\hline \multicolumn{8}{|c|}{ Alhagi sparsifolia } \\
\hline 13.06 .99 & 76.7 & 17.69 & 23.52 & 33643.41 & 60954.74 & 2.19 & 11.003 \\
\hline 23.06 .99 & 45.71 & 19.43 & 23.98 & 33757.41 & 63907.04 & 2.09 & 12.413 \\
\hline \multicolumn{8}{|c|}{ Calligonum caput-medusae } \\
\hline 20.07 .99 & 96.3 & 25.54 & 23.13 & 36538.70 & 74479.00 & 1.29 & 8.141 \\
\hline 23.07 .99 & 84.4 & 24.26 & 24.52 & 29757.97 & 60525.76 & 1.56 & 7.372 \\
\hline \multicolumn{8}{|c|}{ Tamarix ramosissima } \\
\hline 05.08 .99 & 84.1 & 19.75 & 25.24 & 39398.53 & 82202.35 & 1.69 & 19.276 \\
\hline 08.08 .99 & 84.3 & 13.17 & 26.98 & 42210.66 & 84717.43 & 2.46 & 20.528 \\
\hline
\end{tabular}


Tab. 12: Tagessummen des Saftflusses der untersuchten Arten 1999 und 2000. Mittelwerte (MW) des trockengewichtsbezogenen Saftflusses $\left[\mathrm{g} \mathrm{H}_{2} \mathrm{O}^{*} \mathrm{~g} \mathrm{TG}^{-1 *} \mathrm{Tag}^{-1}\right]$ von 6 Pflanzen mit Standardfehler (se). Die Tage sind als Juliantage angegeben.

\begin{tabular}{|c|c|c|c|c|c|c|c|c|c|c|c|c|c|c|}
\hline \multicolumn{15}{|c|}{ Alhagi sparsifolia 1999} \\
\hline Tag & MW & se & Tag & MW & se & Tag & MW & se & Tag & MW & se & Tag & MW & se \\
\hline 121 & 21.43 & 2.60 & 152 & 10.81 & 1.29 & 183 & 13.66 & 1.16 & 214 & 14.42 & 0.99 & 245 & 13.17 & 1.21 \\
\hline 122 & 27.88 & 3.28 & 153 & 10.53 & 1.31 & 184 & 13.31 & 1.13 & 215 & 8.85 & 1.25 & 246 & 13.79 & 1.09 \\
\hline 123 & 22.70 & 2.89 & 154 & 9.85 & 1.22 & 185 & 9.85 & 0.83 & 216 & 10.42 & 1.00 & 247 & 13.62 & 1.24 \\
\hline 124 & 22.59 & & 155 & 10.23 & 1.27 & 186 & 11.54 & 0.89 & 217 & 13.47 & & 248 & & \\
\hline 125 & 21.11 & 2.94 & 156 & 10.55 & 1.35 & 187 & 12.44 & 0.99 & 218 & 14.58 & 1.09 & 249 & 10.88 & 1.10 \\
\hline 126 & 24.01 & 3.51 & 157 & 12.21 & 1.48 & 188 & 13.10 & 1.16 & 219 & 13.84 & 0.91 & 250 & 14.10 & 1.25 \\
\hline 127 & 22.07 & 3.30 & 158 & 13.24 & 1.64 & 189 & 13.28 & 1.14 & 220 & 13.70 & 0.89 & 251 & 13.21 & 1.19 \\
\hline 128 & 24.74 & 3.50 & 159 & 13.70 & 1.72 & 190 & 13.52 & 1.20 & 221 & 14.10 & 1.3 & 252 & 11.74 & 1.1 \\
\hline 129 & 25.99 & 3.42 & 160 & 13.15 & 1.66 & 191 & 13.89 & 1.28 & 222 & 13.05 & 1.34 & 253 & 13.51 & 1.21 \\
\hline 130 & 23.98 & 3.11 & 161 & 12.27 & 1.47 & 192 & 12.53 & 1.26 & 223 & 12.43 & 0.98 & 254 & 13.27 & 1.2 \\
\hline 131 & 30.26 & 3.62 & 162 & 8.03 & 1.09 & 193 & 8.61 & 0.77 & 224 & 12.14 & 0.97 & 255 & 13.62 & 1.22 \\
\hline 132 & 27.76 & 3.19 & 163 & 11.32 & 1.42 & 194 & 12.35 & 1.03 & 225 & 9.58 & 0.81 & 256 & 14.01 & 1.24 \\
\hline & 22.6 & 2.5 & & 11.00 & 1.36 & 195 & 12.94 & 1.13 & 226 & 12.73 & 1.0 & 257 & & 1.2 \\
\hline 134 & 31.65 & 3.28 & 165 & 13.72 & 1.70 & 196 & 13.36 & 1.25 & 227 & 13. & 1.0 & 258 & 13.98 & 1.24 \\
\hline 135 & 26.57 & 2.75 & 166 & 14.12 & 1.62 & 197 & 14.79 & 1.31 & 228 & 13.55 & 1.07 & 259 & 13.36 & 1.01 \\
\hline 136 & 28.41 & 3.00 & 167 & 14.38 & 1.59 & 198 & 13.99 & 1.17 & 229 & 12.88 & 1.02 & 260 & 13.27 & 1.00 \\
\hline 1 & & 2 & 168 & & 1.32 & 199 & & 1.16 & 230 & & 0.8 & 261 & & $1 .($ \\
\hline 138 & 22.98 & 2.74 & 169 & 13.54 & 1.44 & 200 & 12.19 & 1.12 & 231 & 12.75 & 1.01 & 262 & 13.14 & 1.00 \\
\hline 139 & 23.01 & 2.65 & 170 & 13.25 & 1.41 & 201 & 11.08 & 0.96 & 232 & 12.73 & 1.01 & 263 & 12.35 & 0.95 \\
\hline 140 & 21.84 & 2.51 & 171 & 12.18 & 1.29 & 202 & 14.58 & 1.16 & 233 & 12.17 & 0.97 & 264 & 12.64 & 0.97 \\
\hline 141 & & 3.18 & 172 & 12.08 & 1.24 & 203 & 11.83 & 1.16 & 234 & 12.10 & 0.95 & 265 & & 1.0 \\
\hline 142 & & 2.7 & 173 & & 1.22 & 204 & 11.43 & 0.94 & 235 & 13. & & 266 & & 1.1 \\
\hline 143 & 20.52 & 2.52 & 174 & 12.41 & 1.14 & 205 & 13.85 & 1.11 & 236 & 13.12 & 1.03 & 267 & 11.57 & 1.91 \\
\hline 144 & 17.49 & 2.17 & 175 & 12.92 & 1.34 & 206 & 13.34 & 1.30 & 237 & 13.25 & 0.89 & 268 & 11.56 & 1.95 \\
\hline 145 & 14.82 & 1.90 & 176 & 12.29 & 1.30 & 207 & 13.76 & 1.06 & 238 & 12.94 & 0.76 & 269 & 10.78 & 1.84 \\
\hline 146 & 12.40 & 1.6 & 177 & 11.6 & 1.23 & 208 & 13.88 & 1.13 & 239 & 12. & 0.67 & 270 & 10.86 & 1.86 \\
\hline 147 & 14.79 & 1.77 & 178 & 13.06 & 1.29 & 209 & 12.28 & 1.14 & 240 & 12.70 & 0.79 & 271 & 9.88 & 1.62 \\
\hline 148 & 16.06 & 1.93 & 179 & 13.03 & 1.27 & 210 & 11.30 & 1.19 & 241 & 13.60 & 0.85 & 272 & 10.16 & 1.68 \\
\hline 149 & 13.17 & 1.71 & 180 & 12.99 & 1.19 & 211 & 14.22 & 1.35 & 242 & & 0.87 & 273 & 8.95 & 1.42 \\
\hline 150 & & 1.62 & 181 & 13.46 & 1.17 & 212 & 12.82 & 1.17 & 243 & 13. & 0.89 & 274 & 10.32 & 1.70 \\
\hline 151 & & 1.46 & 02 & & .00 & 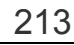 & & & & 13.41 & 1.00 & 215 & 09 & 1.19 \\
\hline
\end{tabular}

\begin{tabular}{|c|c|c|c|c|c|c|c|c|c|c|c|c|c|c|}
\hline \multicolumn{15}{|c|}{ Alhagi sparsifolia 2000} \\
\hline Tag & MW & se & Tag & MW & se & Tag & MW & se & Tag & MW & se & Tag & MW & se \\
\hline 131 & 16.68 & 3.66 & 148 & 14.57 & 4.46 & 165 & 14.74 & 5.34 & 182 & 9.16 & 3.14 & 199 & 6.95 & 2.49 \\
\hline 132 & 20.02 & 5.01 & 149 & 14.12 & 4.33 & 166 & 14.54 & 5.45 & 183 & 10.02 & 3.18 & 200 & 7.79 & 2.70 \\
\hline 133 & 19.36 & 4.97 & 150 & 18.86 & 5.82 & 167 & 13.65 & 5.14 & 184 & 8.10 & 2.89 & 201 & 5.65 & 2.02 \\
\hline 134 & 15.99 & 3.87 & 151 & 20.57 & 7.32 & 168 & 12.52 & 4.88 & 185 & 9.57 & 3.35 & 202 & 6.85 & 2.37 \\
\hline 135 & 16.21 & 0.59 & 152 & 17.94 & 5.73 & 169 & 13.22 & 5.31 & 186 & 8.40 & 2.96 & 203 & 6.63 & 2.30 \\
\hline 136 & 18.20 & 1.15 & 153 & 20.28 & 6.74 & 170 & 12.19 & 4.70 & 187 & 8.95 & 3.15 & 204 & 6.96 & 2.50 \\
\hline 137 & 21.10 & 2.61 & 154 & 18.62 & 6.11 & 171 & 12.27 & 4.66 & 188 & 8.37 & 2.83 & 205 & 7.46 & 2.60 \\
\hline 138 & 21.63 & 3.91 & 155 & 17.20 & 5.92 & 172 & 10.57 & 3.62 & 189 & 8.66 & 3.09 & 206 & 6.43 & 2.23 \\
\hline 139 & 23.96 & 5.58 & 156 & 17.52 & 5.82 & 173 & 12.53 & 4.42 & 190 & 8.96 & 3.19 & 207 & 6.43 & 2.24 \\
\hline 140 & 22.61 & 5.71 & 157 & 21.58 & 7.48 & 174 & 15.05 & 5.50 & 191 & 8.71 & 2.99 & 208 & 7.05 & 2.43 \\
\hline 141 & 17.24 & 5.03 & 158 & 19.23 & 6.76 & 175 & 13.99 & 4.84 & 192 & .53 & 3.02 & 209 & 6.57 & 2.30 \\
\hline 142 & 18.8 & 5.7 & 159 & 19.24 & 7.01 & 176 & 12.4 & 4.44 & 193 & & 3. & 210 & 6.57 & \\
\hline 143 & 18.05 & 5.56 & 160 & 14.09 & 4.69 & 177 & 12.90 & 4.62 & 194 & 8.40 & 3.00 & 211 & 7.67 & 2.65 \\
\hline 144 & 10.62 & 2.57 & 161 & 16.39 & 5.77 & 178 & 12.60 & 4.56 & 195 & 8.10 & 2.85 & 212 & 7.62 & 2.63 \\
\hline 145 & 12.46 & 3.33 & 162 & 16.61 & 6.34 & 179 & 12.10 & 4.42 & 196 & 8.01 & 2.77 & & & \\
\hline 146 & 13.0 & 3.6 & 163 & 16.27 & 6.35 & 180 & 11.71 & 4.62 & 197 & 7.44 & 2.6 & & & \\
\hline 147 & 12.65 & 3.60 & 164 & 16.27 & 6.17 & 181 & 8.75 & 3.27 & 198 & 7.76 & 2.69 & & alog & \\
\hline
\end{tabular}




\begin{tabular}{|c|c|c|c|c|c|c|c|c|c|c|c|c|c|c|}
\hline \multicolumn{15}{|c|}{ Calligonum caput-medusae 1999} \\
\hline Tag & MW & se & Tag & MW & se & Tag & MW & se & Tag & MW & se & Tag & MW & se \\
\hline 103 & 39.39 & 4.07 & 139 & 14.10 & 2.67 & 175 & 8.73 & 1.68 & 211 & 6.41 & 1.31 & 247 & 11.03 & 2.43 \\
\hline 104 & 35.94 & 4.20 & 140 & 14.08 & 2.69 & 176 & 9.90 & 1.85 & 212 & 8.16 & 1.63 & 248 & 11.02 & 2.41 \\
\hline 105 & 38.29 & 4.80 & 141 & 15.22 & 3.04 & 177 & 9.98 & 1.87 & 213 & 10.40 & 2.61 & 249 & 8.15 & 1.86 \\
\hline 106 & 39.75 & 5.10 & 142 & 13.39 & 2.65 & 178 & 10.44 & 2.00 & 214 & 10.86 & 2.70 & 250 & 10.86 & 2.49 \\
\hline 107 & 34.87 & & 143 & & 2.69 & 179 & 10.65 & 2.13 & 215 & 4.22 & & 251 & & 2.36 \\
\hline 108 & 34.93 & 4.04 & 144 & 11.14 & 2.21 & 180 & 9.74 & 1.96 & 216 & 9.02 & 2.47 & 252 & 10.85 & 2.53 \\
\hline 109 & 39.08 & 5.19 & 145 & 9.27 & 1.90 & 181 & 10.31 & 2.02 & 217 & 10.82 & 2.65 & 253 & 10.68 & 2.44 \\
\hline 110 & 39.43 & 5.76 & 146 & 7.32 & 1.38 & 182 & 9.58 & 1.97 & 218 & 10.94 & 2.65 & 254 & 12.06 & 2.60 \\
\hline 111 & 36.37 & 5.06 & 147 & 10.25 & 1.51 & 183 & & 1.96 & 219 & 11.75 & 2.8 & 255 & & \\
\hline 112 & 38.95 & 5.41 & 148 & 12.21 & 1.94 & 184 & 9.16 & 1.91 & 220 & 11.53 & 2.79 & 256 & 12.34 & 2.63 \\
\hline 113 & 30.09 & 4.98 & 149 & 11.85 & 1.73 & 185 & 5.78 & 1.26 & 221 & 8.44 & 2.35 & 257 & 11.31 & 2.40 \\
\hline 114 & 15.92 & 2.36 & 150 & 11.14 & 1.68 & 186 & 7.62 & 1.79 & 222 & 8.39 & 2.33 & 258 & 11.46 & 2.52 \\
\hline 115 & 6.98 & 0.73 & 151 & 11.75 & 1.80 & 187 & 8.68 & 1.95 & 223 & 10.06 & 2.58 & 259 & 11.19 & 2.43 \\
\hline 116 & 7.55 & 0.46 & 152 & 12.39 & 1.89 & 188 & 9.33 & 2.19 & 224 & 11.26 & 2.87 & 260 & 10.92 & 2.34 \\
\hline 117 & 8.70 & 0.67 & 153 & 12.78 & 1.98 & 189 & 9. & 2.15 & 225 & 4.77 & 1.13 & 261 & & 2.35 \\
\hline 118 & 9.45 & 0.93 & 154 & 12.18 & 2.02 & 190 & 10.15 & 2.34 & 226 & 9.54 & 2.09 & 262 & 10.75 & 2.42 \\
\hline 119 & 12.73 & 1.49 & 155 & 12.01 & 2.03 & 191 & 10.13 & 2.27 & 227 & 10.47 & 2.69 & 263 & 11.28 & 2.69 \\
\hline 120 & & & & & 1.96 & 192 & & & & & 2. & & & \\
\hline 121 & 9.19 & 1.92 & 157 & 10.98 & 1.90 & 193 & 4.18 & 0.80 & 229 & 10.81 & 2.77 & 265 & 10.07 & 2.42 \\
\hline 122 & 12.84 & 1.73 & 158 & 12.01 & 2.05 & 194 & 7.31 & 1.62 & 230 & 10.83 & 2.78 & 266 & 9.45 & 2.23 \\
\hline 123 & 14.69 & 2.06 & 159 & 12.87 & 2.15 & 195 & 8.74 & 1.98 & 231 & 10.68 & 2.46 & 267 & 9.15 & 2.16 \\
\hline 124 & 15.2 & 2.1 & 160 & 12. & 1.98 & 196 & & 1.88 & 232 & & 2.71 & 268 & 35 & 2.7 \\
\hline 125 & 15.38 & 2.33 & 161 & 10.66 & 1.78 & 197 & 9.88 & 2.13 & 233 & 11.86 & 3.25 & 269 & 8.56 & 2.04 \\
\hline 126 & 17.73 & 2.89 & 162 & 6.33 & 1.14 & 198 & 9.99 & 2.08 & 234 & 11.21 & 2.98 & 270 & 8.26 & 1.98 \\
\hline 127 & 17.01 & 2.94 & 163 & 9.31 & 1.70 & 199 & 10.55 & 2.14 & 235 & 11.63 & 2.79 & 271 & 7.96 & 1.92 \\
\hline 128 & 16.6 & 3.12 & 164 & 9.07 & 1.62 & 200 & & 1.45 & 236 & 11.38 & 2.8 & 272 & 66 & 1.8 \\
\hline 129 & 16.25 & 00 & 165 & 10.09 & 1.88 & 201 & 8.88 & 1.66 & 237 & 11.08 & 2.85 & 273 & 7.36 & 1.80 \\
\hline 130 & 15.23 & 2.80 & 166 & 10.70 & 1.77 & 202 & 8.58 & 1.93 & 238 & 10.75 & 2.68 & 274 & 7.06 & 1.74 \\
\hline 131 & 16.29 & 3.09 & 167 & 11.21 & 1.80 & 203 & 5.54 & 1.18 & 239 & 9.58 & 2.50 & 275 & 6.76 & 1.68 \\
\hline 132 & 17.35 & 3.2 & 168 & 9.47 & 1.49 & 20 & 6.1 & 1.27 & 240 & 10.97 & 2.70 & 276 & 46 & 1.6 \\
\hline 133 & & 1.7 & 16 & 9.5 & 1.69 & 205 & & 1.65 & 241 & 11.65 & 2.78 & 277 & 6.17 & 1.76 \\
\hline 134 & 12.73 & 2.20 & 170 & 9.40 & 1.59 & 206 & 6.75 & 1.36 & 242 & 11.57 & 2.76 & 278 & 6.96 & 1.73 \\
\hline 135 & 12.36 & 2.09 & 171 & 8.14 & 1.40 & 207 & 8.26 & 1.61 & 243 & 11.57 & 2.76 & 279 & 7.19 & 1.82 \\
\hline 136 & 15.64 & 2.83 & 172 & 7.00 & 1.23 & 208 & 8.5 & 1.66 & 244 & 9.71 & 2.27 & 280 & 6.93 & 1.75 \\
\hline 137 & 15.92 & 3.17 & 173 & 8.13 & 1.48 & 209 & 7.6 & 1.58 & 245 & 7.76 & 1.89 & & & \\
\hline 138 & 14.15 & 2.76 & 174 & 7.37 & 1.43 & 210 & 5.28 & 1.09 & 246 & 10.18 & 2.30 & & & \\
\hline
\end{tabular}

\begin{tabular}{|c|c|c|c|c|c|c|c|c|c|c|c|c|c|c|}
\hline \multicolumn{15}{|c|}{ Calligonum caput-medusae 2000} \\
\hline Tag & MW & se & Tag & MW & se & Tag & MW & se & Tag & MW & se & Tag & MW & se \\
\hline 121 & 30.18 & 7.40 & 134 & 21.95 & 5.71 & 147 & 12.90 & 2.60 & 160 & 7.66 & 1.43 & 173 & 6.36 & 1.23 \\
\hline 122 & 30.99 & 7.69 & 135 & 15.55 & 3.86 & 148 & 18.44 & 4.57 & 161 & 11.31 & 2.29 & 174 & 8.38 & 1.72 \\
\hline 123 & 29.66 & 7.37 & 136 & 18.12 & 3.94 & 149 & 18.99 & 4.49 & 162 & 11.58 & 2.40 & 175 & 11.11 & 2.28 \\
\hline 124 & 27.52 & 6.68 & 137 & 19.72 & 4.39 & 150 & 20.25 & 5.42 & 163 & 10.73 & 1.91 & 176 & 10.10 & 2.06 \\
\hline 125 & 25.73 & 6.15 & 138 & 21.86 & 4.94 & 151 & 14.11 & 2.81 & 164 & 11.37 & 2.22 & 177 & 10.50 & 2.08 \\
\hline 126 & 20.58 & 3.90 & 139 & 22.60 & 5.53 & 152 & 10.36 & 2.47 & 165 & 11.46 & 2.32 & 178 & 10.83 & 2.20 \\
\hline 127 & 26.42 & 6.39 & 140 & 21.64 & 5.45 & 153 & 12.58 & 3.03 & 166 & 11.25 & 2.37 & 179 & 10.72 & 2.19 \\
\hline 128 & 26.53 & 6.08 & 141 & 21.12 & 4.73 & 154 & 11.26 & 2.39 & 167 & 11.02 & 2.34 & 180 & 10.40 & 2.11 \\
\hline 129 & 24.14 & 5.57 & 142 & 23.40 & 6.01 & 155 & 8.32 & 2.12 & 168 & 10.05 & 2.00 & 181 & 7.81 & 1.48 \\
\hline 130 & 24.32 & 5.24 & 143 & 22.99 & 6.00 & 156 & 8.03 & 1.55 & 169 & 10.38 & 2.04 & & & \\
\hline 131 & 26.40 & 6.96 & 144 & 13.96 & 2.75 & 157 & 12.19 & 2.00 & 170 & 9.81 & 1.87 & & & \\
\hline 132 & 29.91 & 9.37 & 145 & 15.06 & 3.40 & 158 & 11.09 & 2.05 & 171 & 10.33 & 1.99 & & & \\
\hline 133 & 28.36 & 7.98 & 146 & 13.98 & 2.96 & 159 & 11.18 & 2.30 & 172 & 10.39 & 2.03 & & talogge & \\
\hline
\end{tabular}




\begin{tabular}{|c|c|c|c|c|c|c|c|c|c|c|c|c|c|c|}
\hline \multicolumn{15}{|c|}{ Tamarix ramosissima 1999} \\
\hline Tag & MW & se & Tag & MW & se & Tag & MW & se & Tag & MW & se & Tag & MW & se \\
\hline 103 & 3.41 & 0.65 & 139 & 18.44 & 2.50 & 175 & 19.98 & 2.83 & 211 & 12.83 & 2.45 & 247 & 15.30 & 2.36 \\
\hline 104 & 10.49 & 1.84 & 140 & 19.07 & 2.42 & 176 & 21.23 & 3.10 & 212 & 15.71 & 3.25 & 248 & 15.87 & 2.42 \\
\hline 105 & 10.13 & 1.79 & 141 & 22.05 & 2.85 & 177 & 20.52 & 3.00 & 213 & 16.93 & 3.31 & 249 & 12.18 & 1.59 \\
\hline 106 & 9.58 & 1.70 & 142 & 20.16 & 2.58 & 178 & 21.78 & 3.12 & 214 & 17.48 & 3.36 & 250 & 14.60 & 2.30 \\
\hline 107 & 18.41 & & 143 & 19.96 & 2.56 & 179 & 21.38 & 3.31 & 215 & 6.10 & & 251 & & \\
\hline 108 & 7.96 & 1.67 & 144 & 17.61 & 2.34 & 180 & 21.42 & 2.92 & 216 & 10.20 & 1.98 & 252 & 15.04 & 2.10 \\
\hline 109 & 8.96 & 1.62 & 145 & 13.74 & 1.80 & 181 & 21.66 & 3.10 & 217 & 13.76 & 3.05 & 253 & 15.60 & 2.45 \\
\hline 110 & 6.27 & 0.29 & 146 & 11.00 & 1.42 & 182 & 19.63 & 2.69 & 218 & 15.32 & 3.37 & 254 & 14.77 & 2.11 \\
\hline 111 & 7.49 & 1.32 & 147 & 14.72 & 1.85 & 183 & 20.86 & 2.81 & 219 & 15.21 & 3.3 & 255 & 15.26 & 2.3 \\
\hline 112 & 7.51 & 0.87 & 148 & 17.30 & 2.07 & 184 & 20.26 & 2.65 & 220 & 16.17 & 3.31 & 256 & 15.94 & 2.52 \\
\hline 113 & 6.39 & 0.45 & 149 & 17.43 & 2.05 & 185 & 15.03 & 1.74 & 221 & 13.72 & 2.48 & 257 & & 2.4 \\
\hline 114 & 5.02 & 0.24 & 150 & 17.29 & 1.95 & 186 & 17.41 & 2.26 & 222 & 14.53 & 2.60 & 258 & 16.11 & 2.50 \\
\hline 115 & 4.00 & 0.33 & 151 & 17.25 & 2.00 & 187 & 19.28 & 2.53 & 223 & 15.01 & 2.67 & 259 & 15.44 & 2.30 \\
\hline 116 & 3.84 & 0.36 & 152 & 17.49 & 2.17 & 188 & 20.49 & 2.70 & 224 & 15.45 & 2.61 & 260 & 14.87 & 2.23 \\
\hline 117 & & 41 & 153 & 15 & 2.14 & 189 & 19. & 2.78 & 225 & 66 & 0.78 & 261 & & 2.3 \\
\hline 118 & 4.13 & 0.52 & 154 & 17.35 & 1.86 & 190 & 20.53 & 3.02 & 226 & 13.66 & 2.32 & 262 & 14.93 & 2.14 \\
\hline 119 & 5.00 & 1.05 & 155 & 18.20 & 1.99 & 191 & 21.03 & 3.06 & 227 & 14.50 & 2.76 & 263 & 14.21 & 2.08 \\
\hline 120 & 6.08 & 1.50 & 156 & 18.09 & 2.03 & 192 & 15.02 & 1.80 & 228 & 14.76 & 2.82 & 264 & 14.61 & 2.1 \\
\hline 121 & 3.20 & 0.82 & 157 & 17.52 & 1.94 & 193 & 11. & 1.28 & 229 & 15. & 2.8 & 265 & & 2.12 \\
\hline 122 & 4.32 & 1.19 & 158 & 19.55 & 2.24 & 194 & 15.23 & 2.04 & 230 & 15.02 & 2.79 & 266 & 14.46 & 2.11 \\
\hline 123 & 6.17 & 1.61 & 159 & 19.88 & 2.31 & 195 & 18.86 & 2.63 & 231 & 14.92 & 2.74 & 267 & 14.76 & 2.18 \\
\hline 124 & & 1 & 160 & 18.84 & 2.16 & 196 & 18. & 2.23 & 232 & 14. & 2.40 & 268 & & 2.1 \\
\hline 125 & 7.87 & 1.7 & 161 & 16.41 & 1.82 & 197 & 20. & 2.84 & 233 & 14. & & 269 & & 2. \\
\hline 126 & 10.30 & 2.05 & 162 & 13.00 & 1.43 & 198 & 21.04 & 2.89 & 234 & 15.23 & 2.59 & 270 & 13.74 & 2.12 \\
\hline 127 & 11.23 & 2.02 & 163 & 18.23 & 2.22 & 199 & 20.84 & 2.79 & 235 & 16.09 & 2.81 & 271 & 12.26 & 1.90 \\
\hline 128 & 12.88 & 2.17 & 164 & 17.99 & 2.00 & 200 & 15. & 1.81 & 236 & 15.75 & 2.66 & 272 & 11.48 & 1.7 \\
\hline 129 & 14.0 & 2.1 & 16 & 20.4 & 2.61 & 201 & 16. & 1.96 & 237 & & 2.5 & 273 & & \\
\hline 130 & 13.76 & 2.05 & 166 & 20.70 & 2.61 & 202 & 18.50 & 2.50 & 238 & 14.45 & 2.35 & 274 & 11.62 & 1.74 \\
\hline 131 & 16.06 & 2.37 & 167 & 21.09 & 2.66 & 203 & 14.63 & 1.76 & 239 & 14.76 & 2.28 & 275 & 10.02 & 1.31 \\
\hline 132 & 17.66 & 2.68 & 168 & 19.04 & 2.32 & 204 & 15.24 & 1.92 & 240 & 14. & 2.48 & 276 & & 1.5 \\
\hline 133 & 8.2 & 1.1 & 169 & 20.6 & 2.93 & 205 & 18. & 2.57 & 241 & 16.45 & 2.4 & 277 & & 1.4 \\
\hline 134 & 12.51 & 1.64 & 170 & 20.73 & 2.83 & 206 & 16.66 & 2.04 & 242 & 16.56 & 2.70 & 278 & 11.09 & 1.71 \\
\hline 135 & 12.99 & 1.75 & 171 & 18.46 & 2.41 & 207 & 17.58 & 2.48 & 243 & 16.47 & 2.70 & 279 & 10.69 & 1.66 \\
\hline 136 & 16.76 & 2.40 & 172 & 17.60 & 2.15 & 208 & 16.50 & 3.18 & 244 & 13.23 & 1.86 & & & \\
\hline 137 & 19.07 & 2.5 & 173 & & 2.41 & 209 & 13. & 2.79 & 245 & & 1.5 & & & \\
\hline 138 & 17.62 & 2.33 & 174 & 18.40 & 2.33 & 210 & 10.55 & 1.91 & 246 & 14.53 & & & & \\
\hline
\end{tabular}

\section{Tamarix ramosissima 2000}

\begin{tabular}{rcc|ccc|ccc|ccc|ccc}
\hline Tag & MW & se & Tag & MW & se & Tag & MW & se & Tag & MW & se & Tag & MW & se \\
\hline 121 & $\mathbf{8 1 . 8 2}$ & 4.29 & 135 & $\mathbf{5 1 . 8 3}$ & 3.04 & 149 & 57.87 & 6.90 & 178 & $\mathbf{5 . 7 3}$ & 0.64 & 192 & $\mathbf{2 5 . 8 5}$ & 2.06 \\
122 & $\mathbf{6 9 . 8 1}$ & 3.65 & 136 & $\mathbf{5 8 . 3 7}$ & 3.28 & 150 & 56.56 & 7.63 & 179 & $\mathbf{1 2 . 3 8}$ & 1.27 & 193 & $\mathbf{2 6 . 0 2}$ & 2.04 \\
123 & $\mathbf{6 7 . 0 1}$ & 4.00 & 137 & $\mathbf{5 7 . 7 8}$ & 3.40 & 151 & 59.31 & 7.50 & 180 & $\mathbf{1 3 . 0 7}$ & 1.01 & 194 & $\mathbf{2 7 . 4 0}$ & 2.48 \\
124 & $\mathbf{7 1 . 2 7}$ & 5.44 & 138 & $\mathbf{5 9 . 8 0}$ & 3.64 & 152 & 49.78 & 7.73 & 181 & $\mathbf{1 4 . 0 6}$ & 1.23 & 195 & $\mathbf{3 1 . 1 5}$ & 2.17 \\
125 & $\mathbf{6 5 . 8 4}$ & 4.51 & 139 & $\mathbf{5 7 . 7 3}$ & 3.65 & 153 & 44.40 & 8.81 & 182 & $\mathbf{1 3 . 3 8}$ & 1.05 & 196 & $\mathbf{3 1 . 2 2}$ & 2.31 \\
126 & $\mathbf{6 6 . 5 4}$ & 3.90 & 140 & $\mathbf{5 5 . 4 3}$ & 3.42 & 154 & 36.97 & 7.56 & 183 & $\mathbf{1 2 . 7 0}$ & 0.37 & 197 & $\mathbf{2 8 . 7 6}$ & 1.85 \\
127 & $\mathbf{7 2 . 3 4}$ & 5.03 & 141 & $\mathbf{5 9 . 4 4}$ & 2.80 & 155 & 30.69 & 5.58 & 184 & $\mathbf{1 4 . 3 0}$ & 0.60 & 198 & $\mathbf{2 9 . 4 5}$ & 2.51 \\
128 & $\mathbf{7 3 . 8 2}$ & 4.67 & 142 & $\mathbf{6 2 . 3 8}$ & 3.67 & 156 & 19.27 & 2.12 & 185 & $\mathbf{1 3 . 5 7}$ & 0.44 & 199 & $\mathbf{2 5 . 9 1}$ & 2.17 \\
129 & $\mathbf{6 8 . 9 1}$ & 4.08 & 143 & $\mathbf{6 2 . 1 2}$ & 3.60 & 157 & 18.80 & 2.15 & 186 & $\mathbf{1 6 . 2 8}$ & 0.48 & 200 & $\mathbf{2 5 . 4 5}$ & 2.38 \\
130 & $\mathbf{6 9 . 2 1}$ & 4.48 & 144 & $\mathbf{6 3 . 8 9}$ & 3.35 & 158 & 16.45 & 1.46 & 187 & $\mathbf{1 6 . 0 0}$ & 0.54 & 201 & $\mathbf{2 6 . 2 7}$ & 2.25 \\
131 & $\mathbf{6 6 . 4 1}$ & 4.78 & 145 & $\mathbf{5 4 . 5 7}$ & 2.93 & 159 & 15.99 & 1.36 & 188 & $\mathbf{1 7 . 9 4}$ & 0.79 & 202 & $\mathbf{1 4 . 6 0}$ & 1.16 \\
132 & $\mathbf{7 1 . 6 2}$ & 4.55 & 146 & $\mathbf{5 2 . 5 4}$ & 3.58 & 160 & 7.91 & 0.77 & 189 & $\mathbf{2 0 . 2 5}$ & 0.86 & & & \\
133 & $\mathbf{7 2 . 4 3}$ & 4.86 & 147 & $\mathbf{5 1 . 3 2}$ & 3.86 & Ausfall des & 190 & $\mathbf{1 2 . 7 3}$ & 1.27 & Ausfall des \\
134 & $\mathbf{6 9 . 4 0}$ & 3.40 & 148 & $\mathbf{5 4 . 9 9}$ & 5.27 & Dataloggers & 191 & $\mathbf{1 3 . 7 1}$ & 1.57 & Dataloggers \\
\hline
\end{tabular}




\begin{tabular}{|c|c|c|c|c|c|c|c|c|c|c|c|c|c|c|}
\hline \multicolumn{15}{|c|}{ Populus euphratica 1999} \\
\hline Tag & MW & se & Tag & MW & se & Tag & MW & se & Tag & MW & se & Tag & MW & se \\
\hline 119 & 107.6 & 48.6 & 141 & 0.39 & 0.18 & 163 & 1.20 & 0.36 & 224 & 1.05 & 0.31 & 246 & 1.51 & 0.32 \\
\hline 120 & 19.26 & 6.20 & 142 & 0.38 & 0.19 & 164 & 1.85 & 0.24 & 225 & 0.99 & 0.27 & 247 & 1.87 & 0.51 \\
\hline 121 & 15.35 & 5.88 & 143 & 0.29 & 0.11 & 165 & 1.34 & 0.73 & 226 & 1.38 & 0.42 & 248 & 2.01 & 0.52 \\
\hline 122 & 25.95 & 19.2 & 144 & 0.27 & 0.08 & 166 & 0.62 & 0.37 & 227 & 1.20 & 0.34 & 249 & 1.75 & 0.50 \\
\hline 123 & 10.49 & 6.46 & 145 & 0.28 & 0.08 & 167 & 1.61 & 0.96 & 228 & 1.23 & 0.36 & 250 & 1.80 & 0.51 \\
\hline 124 & 6.60 & 3.87 & 146 & 0.27 & 0.09 & 168 & 1.57 & 1.40 & 229 & 1.13 & 0.32 & 251 & 1.90 & 0.51 \\
\hline 125 & 3.96 & 1.72 & 147 & 0.31 & 0.09 & 169 & 1.98 & 1.42 & 230 & 1.04 & 0.31 & 252 & 1.99 & 0.52 \\
\hline 126 & 3.62 & 1.81 & 148 & 0.26 & 0.08 & 170 & 1.65 & 1.38 & 231 & 0.97 & 0.31 & 253 & 2.02 & 0.52 \\
\hline 127 & 2.81 & 1.29 & 149 & 0.25 & 0.08 & & & & 232 & 1.00 & 0.31 & 254 & 2.11 & 0.53 \\
\hline 128 & 2.62 & 1.34 & 150 & 0.26 & 0.08 & 211 & 0.98 & 0.31 & 233 & 0.99 & 0.30 & 255 & 2.09 & 0.53 \\
\hline 129 & 2.31 & 1.22 & 151 & 0.24 & 0.08 & 212 & 0.86 & 0.27 & 234 & 1.01 & 0.30 & 256 & 2.10 & 0.53 \\
\hline 130 & 2.87 & 1.8 & 152 & 1.03 & 0.48 & 213 & 1.01 & 0.34 & 235 & & 0.29 & 257 & 2.10 & 0.52 \\
\hline 131 & 3.57 & 2.69 & 153 & 1.53 & 0.12 & 214 & 1.04 & 0.34 & 236 & 0.98 & 0.28 & 258 & 2.07 & 0.52 \\
\hline 132 & 2.01 & 1.21 & 154 & 2.11 & 0.18 & 215 & 1.00 & 0.33 & 237 & 1.05 & 0.28 & 259 & 2.09 & 0.52 \\
\hline 133 & 1.57 & 0.61 & 155 & 2.15 & 0.34 & 216 & 1.20 & 0.34 & 238 & 1.00 & 0.29 & 260 & 2.02 & 0.52 \\
\hline 134 & 1.51 & 0.64 & 156 & 2.33 & 0.73 & 217 & 1.17 & 0.33 & 239 & 1.18 & 0.31 & 261 & 2.15 & 0.53 \\
\hline 135 & 1.57 & 0.8 & 157 & 2.23 & 1.14 & 218 & 1.07 & 0.34 & 240 & 1.55 & 0.40 & 262 & 2.13 & 0.53 \\
\hline 136 & 1.35 & 0.56 & 158 & 2.00 & 0.82 & 219 & 0.98 & 0.33 & 241 & 2.22 & 0.58 & 263 & 2.09 & 0.53 \\
\hline 137 & 1.52 & 0.55 & 159 & 1.78 & 1.19 & 220 & 0.98 & 0.34 & 242 & 2.28 & 0.55 & 264 & 1.92 & 0.51 \\
\hline 138 & 2.88 & 1.80 & 160 & 2.25 & 1.61 & 221 & 1.05 & 0.34 & 243 & 3.42 & 0.96 & 265 & 1.93 & 0.52 \\
\hline 139 & 0.37 & 0.19 & 161 & 1.52 & 0.94 & 222 & 1.22 & 0.36 & 244 & 1.70 & 0.37 & 266 & 1.94 & 0.51 \\
\hline 140 & 0.29 & 0.12 & 162 & 1.24 & 0.46 & 223 & 1.12 & 0.32 & 245 & 1.69 & 0.37 & 267 & 0.77 & 0.23 \\
\hline
\end{tabular}

\begin{tabular}{|c|c|c|c|c|c|c|c|c|c|c|c|c|c|c|}
\hline \multicolumn{15}{|c|}{ Populus euphratica 2000} \\
\hline Tag & MW & se & Tag & MW & se & Tag & MW & se & Tag & MW & se & Tag & MW & se \\
\hline 122 & 1.05 & 0.45 & 153 & 1.87 & 0.06 & 184 & 1.62 & 0.09 & 215 & 1.17 & 0.25 & 246 & 1.29 & 0.33 \\
\hline 123 & 0.89 & 0.29 & 154 & 1.85 & 0.01 & 185 & 1.33 & 0.06 & 216 & 1.27 & 0.30 & 247 & 1.10 & 0.30 \\
\hline 124 & 1.06 & 0.40 & 155 & 1.86 & 0.07 & 186 & 1.38 & 0.04 & 217 & 1.18 & 0.32 & 248 & 1.14 & 0.31 \\
\hline 125 & 1.46 & 0.90 & 156 & 1.58 & 0.04 & 187 & 1.13 & 0.01 & 218 & 1.20 & 0.29 & 249 & 1.07 & 0.29 \\
\hline 126 & 1.57 & 1.13 & 157 & 1.72 & 0.02 & 188 & 1.08 & 0.03 & 219 & 1.20 & 0.28 & 250 & 1.08 & 0.29 \\
\hline 127 & 1.25 & 0.81 & 158 & 1.79 & 0.02 & 189 & 1.33 & 0.04 & 220 & 1.20 & 0.27 & 251 & 1.07 & 0.29 \\
\hline 128 & 1.26 & 0.56 & 159 & 1.79 & 0.02 & 190 & 1.24 & 0.13 & 221 & 1.26 & 0.28 & 252 & 1.13 & 0.30 \\
\hline 129 & 0.98 & 0.39 & 160 & 2.10 & 0.04 & 191 & 1.17 & 0.15 & 222 & 1.18 & 0.34 & 253 & 1.10 & 0.30 \\
\hline 130 & 1.20 & 0.31 & 161 & 1.87 & 0.03 & 192 & 1.14 & 0.21 & 223 & 1.33 & 0.40 & 254 & 0.79 & 0.24 \\
\hline 131 & 1.59 & 0.36 & 162 & 1.82 & 0.02 & 193 & 1.18 & 0.24 & 224 & 1.21 & 0.30 & 255 & 0.77 & 0.24 \\
\hline 132 & 1.52 & 0.34 & 163 & 2.02 & 0.03 & 194 & 1.21 & 0.24 & 225 & 1.28 & 0.33 & 256 & 0.89 & 0.26 \\
\hline 133 & 1.54 & 0.35 & 164 & 1.89 & 0.06 & 195 & 1.27 & 0.28 & 226 & 1.21 & 0.34 & 257 & 0.95 & 0.27 \\
\hline 134 & 1.50 & 0.29 & 165 & 1.74 & 0.08 & 196 & 1.27 & 0.34 & 227 & 1.24 & 0.35 & 258 & 1.01 & 0.28 \\
\hline 135 & 1.97 & 0.61 & 166 & 1.66 & 0.08 & 197 & 1.27 & 0.36 & 228 & 1.17 & 0.33 & 259 & 0.99 & 0.29 \\
\hline 136 & 1.68 & 0.51 & 167 & 1.66 & 0.07 & 198 & 1.16 & 0.23 & 229 & 1.29 & 0.34 & 260 & 0.98 & 0.28 \\
\hline 137 & 1.71 & 0.57 & 168 & 1.76 & 0.03 & 199 & 1.10 & 0.17 & 230 & 1.23 & 0.32 & 261 & 0.99 & 0.28 \\
\hline 138 & 1.66 & 0.55 & 169 & 1.83 & 0.09 & 200 & 1.33 & 0.11 & 231 & 1.84 & 0.46 & 262 & 0.98 & 0.28 \\
\hline 139 & 1.72 & 0.56 & 170 & 1.77 & 0.06 & 201 & 1.10 & 0.19 & 232 & 1.37 & 0.32 & 263 & 1.08 & 0.30 \\
\hline 140 & 1.67 & 0.57 & 171 & 1.65 & 0.07 & 202 & 1.09 & 0.23 & 233 & 1.10 & 0.28 & 264 & 1.10 & 0.30 \\
\hline 141 & 1.99 & 0.65 & 172 & 1.61 & 0.01 & 203 & 1.17 & 0.22 & 234 & 1.22 & 0.32 & 265 & 1.11 & 0.30 \\
\hline 142 & 1.83 & 0.57 & 173 & 1.85 & 0.03 & 204 & 1.08 & 0.20 & 235 & 1.20 & 0.32 & 266 & 1.24 & 0.32 \\
\hline 143 & 1.71 & 0.54 & 174 & 1.66 & 0.03 & 205 & 1.12 & 0.18 & 236 & 1.32 & 0.36 & 267 & 1.01 & 0.28 \\
\hline 144 & 1.94 & 0.55 & 175 & 1.52 & 0.07 & 206 & 1.19 & 0.23 & 237 & 1.28 & 0.36 & 268 & 0.75 & 0.23 \\
\hline 145 & 1.86 & 0.53 & 176 & 1.55 & 0.04 & 207 & 1.06 & 0.06 & 238 & 1.34 & 0.39 & 269 & 0.83 & 0.25 \\
\hline 146 & 1.64 & 0.51 & 177 & 1.55 & 0.04 & 208 & 1.21 & 0.04 & 239 & 1.30 & 0.38 & 270 & 0.90 & 0.26 \\
\hline 147 & 1.52 & 0.47 & 178 & 1.57 & 0.00 & 209 & 1.36 & 0.07 & 240 & 1.27 & 0.18 & 271 & 1.42 & 0.18 \\
\hline 148 & 1.68 & 0.59 & 179 & 1.38 & 0.07 & 210 & 1.17 & 0.05 & 241 & 1.33 & 0.37 & 272 & 0.85 & 0.25 \\
\hline 149 & 1.68 & 0.56 & 180 & 1.52 & 0.00 & 211 & 1.26 & 0.10 & 242 & 1.24 & 0.36 & 273 & 0.88 & 0.26 \\
\hline 150 & 1.72 & 0.57 & 181 & 1.61 & 0.02 & 212 & 1.18 & 0.14 & 243 & 1.15 & 0.32 & 274 & 0.91 & 0.26 \\
\hline 151 & 1.70 & 0.49 & 182 & 1.52 & 0.08 & 213 & 1.22 & 0.20 & 244 & 1.17 & 0.34 & & & \\
\hline 152 & 1.71 & 0.51 & 183 & 1.94 & 0.10 & 214 & 1.15 & 0.20 & 245 & 1.18 & 0.32 & & & \\
\hline
\end{tabular}


Tab. 13: Transpiration von Alhagi sparsifolia 1999.

Mittelwerte aus 18 Messungen (6 Pflanzen mit je 3 Parallelen) mit Standardfehler (se). Die Flächenkürzel bedeuten: A Alhagi sparsifolia, F bewässert (flooded), D unbewässert (dry), N nicht genutzt.

\begin{tabular}{crr|rrr}
\hline \multicolumn{3}{c|}{ Alhagi AFN 15.05.1999 } & \multicolumn{3}{c}{ Alhagi AFN 01.06.1999 } \\
\hline Zeit & $\begin{array}{c}\text { Transpiration } \\
{\left[\mathrm{mmol}^{*} \mathrm{~m}^{-2 *} \mathrm{~s}^{-1}\right]}\end{array}$ & $\begin{array}{c}\text { Transpiration } \\
{\left[\mu \mathrm{mol}^{*} \mathrm{~g}^{-2 *} \mathrm{~s}^{-1}\right]}\end{array}$ & Zeit & $\begin{array}{c}\text { Transpiration } \\
{\left[\mathrm{mmol}^{*} \mathrm{~m}^{-2 *} \mathrm{~s}^{-1}\right]}\end{array}$ & $\begin{array}{l}\text { Transpiration } \\
{\left[\mu \mathrm{mol}^{*} \mathrm{~g}^{-2 *} \mathrm{~s}^{-1}\right]}\end{array}$ \\
\hline $7: 52$ & $\mathbf{0 . 9 7 5} \pm 0.102$ & $\mathbf{8 . 5 5 0} \pm 0.891$ & $10: 54$ & $\mathbf{5 . 4 8 2} \pm 0.477$ & $\mathbf{3 3 . 6 6 9} \pm 5.494$ \\
$10: 15$ & $\mathbf{1 . 9 4 8} \pm 0.342$ & $\mathbf{1 7 . 0 9 1} \pm 3.000$ & $12: 06$ & $\mathbf{6 . 4 5 5} \pm 0.353$ & $\mathbf{3 8 . 0 1 9} \pm 4.622$ \\
$11: 48$ & $\mathbf{8 . 7 2 3} \pm 0.466$ & $\mathbf{7 6 . 5 1 7} \pm 4.086$ & $13: 08$ & $\mathbf{6 . 4 2 0} \pm 0.373$ & $\mathbf{3 6 . 8 2 0} \pm 3.775$ \\
$13: 04$ & $\mathbf{1 3 . 0 6 7} \pm 0.973$ & $\mathbf{1 1 4 . 6 2 5} \pm 8.532$ & $15: 06$ & $\mathbf{3 . 0 3 2} \pm 0.633$ & $\mathbf{1 7 . 2 8 2} \pm 4.204$ \\
$14: 41$ & $\mathbf{1 4 . 7 3 4} \pm 1.870$ & $\mathbf{1 2 9 . 2 4 8} \pm 16.40$ & $16: 14$ & $\mathbf{0 . 6 8 1} \pm 0.052$ & $\mathbf{3 . 7 7 4} \pm 0.335$ \\
$16: 00$ & $\mathbf{1 4 . 9 9 9} \pm 2.427$ & $\mathbf{1 3 1 . 5 6 9} \pm 21.29$ & $17: 14$ & $\mathbf{0 . 5 9 1} \pm 0.027$ & $\mathbf{3 . 4 6 0} \pm 0.354$ \\
$17: 05$ & $\mathbf{1 8 . 6 6 4} \pm 2.743$ & $\mathbf{1 6 3 . 7 1 9} \pm 24.06$ & $18: 17$ & $\mathbf{0 . 4 2 2} \pm 0.033$ & $\mathbf{2 . 3 4 1} \pm 0.201$ \\
$18: 15$ & $\mathbf{1 3 . 8 4 4} \pm 1.982$ & $\mathbf{1 2 1 . 4 3 6} \pm 17.38$ & $19: 22$ & $\mathbf{0 . 3 5 7} \pm 0.029$ & $\mathbf{2 . 0 0 4} \pm 0.183$ \\
$19: 29$ & $\mathbf{1 0 . 6 7 0} \pm 1.157$ & $\mathbf{9 3 . 5 9 6} \pm 10.15$ & & & \\
\hline
\end{tabular}

\begin{tabular}{ccc|ccr}
\hline \multicolumn{3}{c|}{ Alhagi AFN 16.06.1999 } & \multicolumn{3}{c}{ Alhagi AFN 08.07.1999 } \\
\hline Zeit & $\begin{array}{c}\text { Transpiration } \\
{\left[\mathrm{mmol}^{*} \mathrm{~m}^{-2 *} \mathrm{~s}^{-1}\right]}\end{array}$ & $\begin{array}{c}\text { Transpiration } \\
{\left[\mu \mathrm{mol}^{*} \mathrm{~g}^{-2 *} \mathrm{~s}^{-1}\right]}\end{array}$ & Zeit & $\begin{array}{c}\text { Transpiration } \\
{\left[\mathrm{mmol}^{*} \mathrm{~m}^{-2 *} \mathrm{~s}^{-1}\right]}\end{array}$ & $\begin{array}{l}\text { Transpiration } \\
{\left[\mu \mathrm{mol}^{*} \mathrm{~g}^{-* *} \mathrm{~s}^{-1}\right]}\end{array}$ \\
\hline $11: 10$ & $\mathbf{1 . 1 7 0} \pm 0.089$ & $\mathbf{5 . 5 6 1} \pm 0.404$ & $8: 35$ & $\mathbf{0 . 6 9 8} \pm 0.061$ & $\mathbf{5 . 4 1 2} \pm 0.472$ \\
$12: 09$ & $\mathbf{1 . 0 8 4} \pm 0.070$ & $\mathbf{5 . 2 0 9} \pm 0.374$ & $11: 26$ & $\mathbf{1 . 6 3 4} \pm 0.153$ & $\mathbf{1 2 . 6 6 7} \pm 1.188$ \\
$13: 04$ & $\mathbf{0 . 8 0 0} \pm 0.058$ & $\mathbf{3 . 8 3 1} \pm 0.289$ & $13: 03$ & $\mathbf{2 . 2 3 2} \pm 0.172$ & $\mathbf{1 7 . 3 0 3} \pm 1.331$ \\
$15: 36$ & $\mathbf{0 . 4 7 7} \pm 0.023$ & $\mathbf{2 . 2 7 8} \pm 0.131$ & $16: 01$ & $\mathbf{1 . 7 8 9} \pm 0.123$ & $\mathbf{1 3 . 8 7 0} \pm 0.950$ \\
$16: 53$ & $\mathbf{0 . 4 0 0} \pm 0.019$ & $\mathbf{1 . 9 1 8} \pm 0.109$ & $17: 19$ & $\mathbf{1 . 6 6 5} \pm 0.122$ & $\mathbf{1 2 . 9 0 5} \pm 0.948$ \\
$17: 59$ & $\mathbf{0 . 3 0 3} \pm 0.014$ & $\mathbf{1 . 4 3 2} \pm 0.058$ & $18: 42$ & $\mathbf{1 . 4 0 3} \pm 0.113$ & $\mathbf{1 0 . 8 7 5} \pm 0.873$ \\
\hline
\end{tabular}

\begin{tabular}{ccc|ccc}
\hline \multicolumn{3}{c|}{ Alhagi AFN 16.08.1999 } & \multicolumn{3}{c}{ Alhagi AFN 25.09.1999 } \\
\hline Zeit & $\begin{array}{c}\text { Transpiration } \\
{\left[\mathrm{mmol}^{*} \mathrm{~m}^{-2 *} \mathrm{~s}^{-1}\right]}\end{array}$ & $\begin{array}{c}\text { Transpiration } \\
{\left[\mu \mathrm{mol}^{*} \mathrm{~g}^{-2 *} \mathrm{~s}^{-1}\right]}\end{array}$ & Zeit & $\begin{array}{c}\text { Transpiration } \\
{\left[\mathrm{mmol}^{*} \mathrm{~m}^{-2 *} \mathrm{~s}^{-1}\right]}\end{array}$ & $\begin{array}{l}\text { Transpiration } \\
{\left[\mu \mathrm{mol}^{*} \mathrm{~g}^{-2 *} \mathrm{~s}^{-1}\right]}\end{array}$ \\
\hline $8: 14$ & $\mathbf{0 . 1 4 7} \pm 0.014$ & $\mathbf{1 . 2 7 3} \pm 0.155$ & $10: 29$ & $\mathbf{0 . 4 2 1} \pm 0.031$ & $\mathbf{2 . 8 9 2} \pm 0.275$ \\
$10: 32$ & $\mathbf{0 . 5 3 3} \pm 0.034$ & $\mathbf{4 . 4 2 2} \pm 0.377$ & $11: 32$ & $\mathbf{0 . 5 5 0} \pm 0.022$ & $\mathbf{3 . 7 5 0} \pm 0.249$ \\
$11: 42$ & $\mathbf{0 . 8 5 9} \pm 0.040$ & $\mathbf{7 . 0 8 0} \pm 0.510$ & $12: 32$ & $\mathbf{0 . 6 6 9} \pm 0.036$ & $\mathbf{4 . 6 0 2} \pm 0.372$ \\
$12: 51$ & $\mathbf{1 . 0 3 0} \pm 0.051$ & $\mathbf{8 . 5 2 5} \pm 0.649$ & $13: 31$ & $\mathbf{0 . 7 5 4} \pm 0.035$ & $\mathbf{5 . 1 6 9} \pm 0.387$ \\
$15: 07$ & $\mathbf{1 . 0 2 9} \pm 0.052$ & $\mathbf{8 . 4 5 6} \pm 0.624$ & $15: 21$ & $\mathbf{0 . 8 0 4} \pm 0.043$ & $\mathbf{5 . 5 6 4} \pm 0.473$ \\
$16: 21$ & $\mathbf{0 . 9 8 5} \pm 0.046$ & $\mathbf{8 . 0 8 4} \pm 0.571$ & $16: 25$ & $\mathbf{0 . 7 6 0} \pm 0.029$ & $\mathbf{5 . 2 1 1} \pm 0.369$ \\
$17: 28$ & $\mathbf{0 . 8 6 3} \pm 0.036$ & $\mathbf{6 . 9 8 9} \pm 0.423$ & $17: 35$ & $\mathbf{0 . 7 1 3} \pm 0.033$ & $\mathbf{4 . 9 2 5} \pm 0.403$ \\
$18: 34$ & $\mathbf{0 . 7 0 9} \pm 0.026$ & $\mathbf{5 . 7 6 6} \pm 0.346$ & $18: 35$ & $\mathbf{0 . 5 7 5} \pm 0.021$ & $\mathbf{3 . 9 4 8} \pm 0.283$ \\
\hline
\end{tabular}

\begin{tabular}{ccc|ccc}
\hline \multicolumn{3}{c|}{ Alhagi ADN 24.08.1999 } & \multicolumn{3}{c}{ Alhagi ADN 06.10.1999 } \\
\hline Zeit & $\begin{array}{c}\text { Transpiration } \\
{\left[\mathrm{mmol}^{*} \mathrm{~m}^{-2 *} \mathrm{~s}^{-1}\right]}\end{array}$ & $\begin{array}{c}\text { Transpiration } \\
{\left[\mu \mathrm{mol}^{*} \mathrm{~g}^{-2 *} \mathrm{~s}^{-1}\right]}\end{array}$ & Zeit & $\begin{array}{c}\text { Transpiration } \\
{\left[\mathrm{mmol}^{*} \mathrm{~m}^{-2 *} \mathrm{~s}^{-1}\right]}\end{array}$ & $\begin{array}{l}\text { Transpiration } \\
{\left[\mu \mathrm{mol}^{*} \mathrm{~g}^{-2 *} \mathrm{~s}^{-1}\right]}\end{array}$ \\
\hline $10: 44$ & $\mathbf{0 . 6 1 2} \pm 0.033$ & $\mathbf{3 . 4 5 2} \pm 0.210$ & $11: 13$ & $\mathbf{0 . 2 7 2} \pm 0.016$ & $\mathbf{1 . 1 9 9} \pm 0.074$ \\
$11: 51$ & $\mathbf{0 . 8 3 2} \pm 0.045$ & $\mathbf{4 . 6 8 0} \pm 0.270$ & $12: 15$ & $\mathbf{0 . 4 3 5} \pm 0.024$ & $\mathbf{1 . 9 1 5} \pm 0.121$ \\
$12: 52$ & $\mathbf{0 . 8 8 9} \pm 0.061$ & $\mathbf{5 . 0 1 8} \pm 0.376$ & $13: 16$ & $\mathbf{0 . 5 0 6} \pm 0.029$ & $\mathbf{2 . 2 2 8} \pm 0.139$ \\
$15: 09$ & $\mathbf{0 . 7 5 7} \pm 0.051$ & $\mathbf{4 . 2 8 2} \pm 0.324$ & $14: 58$ & $\mathbf{0 . 5 1 6} \pm 0.026$ & $\mathbf{2 . 2 7 6} \pm 0.132$ \\
$16: 37$ & $\mathbf{0 . 5 4 2} \pm 0.032$ & $\mathbf{3 . 0 6 2} \pm 0.206$ & $16: 10$ & $\mathbf{0 . 4 9 7} \pm 0.031$ & $\mathbf{2 . 1 8 3} \pm 0.145$ \\
$17: 50$ & $\mathbf{0 . 6 6 7} \pm 0.030$ & $\mathbf{3 . 7 5 1} \pm 0.181$ & $17: 09$ & $\mathbf{0 . 5 0 6} \pm 0.029$ & $\mathbf{2 . 2 3 6} \pm 0.147$ \\
$18: 48$ & $\mathbf{0 . 5 4 0} \pm 0.029$ & $\mathbf{3 . 0 4 6} \pm 0.190$ & $18: 07$ & $\mathbf{0 . 5 2 8} \pm 0.029$ & $\mathbf{2 . 3 2 4} \pm 0.140$ \\
\hline
\end{tabular}


Tab. 14: Stomatäre Leitfähigkeit von Alhagi sparsifolia 1999.

Mittelwerte aus 18 Messungen (6 Pflanzen mit je 3 Parallelen) mit Standardfehler (se). Die Flächenkürzel bedeuten: A Alhagi sparsifolia, $\mathbf{F}$ bewässert (flooded), D unbewässert (dry), N nicht genutzt.

\begin{tabular}{ccc|rrr}
\hline \multicolumn{3}{c|}{ Alhagi AFN 15.05.1999 } & \multicolumn{3}{c}{ Alhagi AFN 01.06.1999 } \\
\hline Zeit & $\begin{array}{c}\text { Leitfähigkeit } \\
{\left[\mathrm{mmol}^{*} \mathrm{~m}^{-2 *} \mathrm{~s}^{-1}\right]}\end{array}$ & $\begin{array}{c}\text { Leitfähigkeit } \\
{\left[\mathrm{mmol}^{*} \mathrm{~g}^{-2 *} \mathrm{~s}^{-1}\right]}\end{array}$ & Zeit & $\begin{array}{c}\text { Leitfähigkeit } \\
{\left[\mathrm{mmol}^{*} \mathrm{~m}^{-2 *} \mathrm{~s}^{-1}\right]}\end{array}$ & $\begin{array}{l}\text { Leitfähigkeit } \\
{\left[\mathrm{mmol}^{*} \mathrm{~g}^{-2 *} \mathrm{~s}^{-1}\right]}\end{array}$ \\
\hline $7: 52$ & $\mathbf{1 7 5 . 6 0} \pm 30.65$ & $\mathbf{1 . 5 4 0} \pm 0.269$ & $10: 54$ & $\mathbf{1 7 8 . 0 3} \pm 15.98$ & $\mathbf{0 . 3 3 2} \pm 0.047$ \\
$10: 15$ & $\mathbf{1 7 0 . 6 6} \pm 32.58$ & $\mathbf{1 . 4 9 7} \pm 0.286$ & $12: 06$ & $\mathbf{1 5 3 . 1 5} \pm 9.03$ & $\mathbf{0 . 2 7 6} \pm 0.028$ \\
$11: 48$ & $\mathbf{4 1 4 . 5 6} \pm 22.30$ & $\mathbf{3 . 6 3 6} \pm 0.196$ & $13: 08$ & $\mathbf{1 2 8 . 9 1} \pm 7.19$ & $\mathbf{0 . 2 3 4} \pm 0.023$ \\
$13: 04$ & $\mathbf{3 8 4 . 3 3} \pm 32.77$ & $\mathbf{3 . 3 7 1} \pm 0.287$ & $15: 06$ & $\mathbf{5 7 . 8 3 1} \pm 11.44$ & $\mathbf{0 . 1 0 2} \pm 0.022$ \\
$14: 41$ & $\mathbf{3 6 0 . 5 6} \pm 51.34$ & $\mathbf{3 . 1 6 3} \pm 0.450$ & $16: 14$ & $\mathbf{1 5 . 9 2 7} \pm 1.075$ & $\mathbf{0 . 0 3 0} \pm 0.004$ \\
$16: 00$ & $\mathbf{3 1 9 . 1 3} \pm 53.23$ & $\mathbf{2 . 7 9 9} \pm 0.467$ & $17: 14$ & $\mathbf{1 4 . 0 7 8} \pm 0.578$ & $\mathbf{0 . 0 2 7} \pm 0.003$ \\
$17: 05$ & $\mathbf{3 7 2 . 4 4} \pm 59.02$ & $\mathbf{3 . 2 6 7} \pm 0.518$ & $18: 17$ & $\mathbf{1 0 . 9 3 1} \pm 0.761$ & $\mathbf{0 . 0 2 1} \pm 0.002$ \\
$18: 15$ & $\mathbf{3 4 5 . 5 6} \pm 51.59$ & $\mathbf{3 . 0 3 1} \pm 0.453$ & $19: 22$ & $\mathbf{1 1 . 4 3 4} \pm 0.980$ & $\mathbf{0 . 0 2 2} \pm 0.003$ \\
$19: 29$ & $\mathbf{3 0 5 . 0 6} \pm 35.77$ & $\mathbf{2 . 6 7 6} \pm 0.314$ & & & \\
\hline
\end{tabular}

\begin{tabular}{ccc|ccc}
\hline \multicolumn{3}{c|}{ Alhagi AFN 16.06.1999 } & \multicolumn{3}{c}{ Alhagi AFN 08.07.1999 } \\
\hline Zeit & $\begin{array}{c}\text { Leitfähigkeit } \\
{\left[\mathrm{mmol}^{*} \mathrm{~m}^{-2 *} \mathrm{~s}^{-1}\right]}\end{array}$ & $\begin{array}{c}\text { Leitfähigkeit } \\
{\left[\mathrm{mmol}^{*} \mathrm{~g}^{-2 *} \mathrm{~s}^{-1}\right]}\end{array}$ & Zeit & $\begin{array}{l}\text { Leitfähigkeit } \\
{\left[\mathrm{mmol}^{*} \mathrm{~m}^{-2 *} \mathrm{~s}^{-1}\right]}\end{array}$ & $\begin{array}{l}\text { Leitfähigkeit } \\
{\left[\mathrm{mmol}^{*} \mathrm{~g}^{-2 *} \mathrm{~s}^{-1}\right]}\end{array}$ \\
\hline $11: 10$ & $\mathbf{2 9 . 0 1 3} \pm 2.015$ & $\mathbf{0 . 0 7 5} \pm 0.008$ & $8: 35$ & $\mathbf{5 4 . 8 4 6} \pm 4.244$ & $\mathbf{0 . 4 2 5} \pm 0.033$ \\
$12: 09$ & $\mathbf{2 2 . 1 9 2} \pm 1.290$ & $\mathbf{0 . 0 5 8} \pm 0.006$ & $11: 26$ & $\mathbf{3 5 . 4 7 1} \pm 3.416$ & $\mathbf{0 . 2 7 5} \pm 0.026$ \\
$13: 04$ & $\mathbf{1 4 . 3 5 7} \pm 1.228$ & $\mathbf{0 . 0 3 7} \pm 0.004$ & $13: 03$ & $\mathbf{3 9 . 0 9 2} \pm 4.003$ & $\mathbf{0 . 3 0 3} \pm 0.031$ \\
$15: 36$ & $\mathbf{6 . 8 7 8} \pm 0.355$ & $\mathbf{0 . 0 1 7} \pm 0.002$ & $16: 01$ & $\mathbf{2 7 . 7 8 9} \pm 2.081$ & $\mathbf{0 . 2 1 5} \pm 0.016$ \\
$16: 53$ & $\mathbf{5 . 3 8 6} \pm 0.305$ & $\mathbf{0 . 0 1 4} \pm 0.001$ & $17: 19$ & $\mathbf{2 3 . 9 5 9} \pm 1.767$ & $\mathbf{0 . 1 8 6} \pm 0.014$ \\
$17: 59$ & $\mathbf{3 . 8 6 0} \pm 0.215$ & $\mathbf{0 . 0 1 0} \pm 0.001$ & $18: 42$ & $\mathbf{2 2 . 9 7 2} \pm 1.866$ & $\mathbf{0 . 1 7 8} \pm 0.014$ \\
\hline
\end{tabular}

\begin{tabular}{ccc|ccc}
\hline \multicolumn{3}{c|}{ Alhagi AFN 16.08.1999 } & \multicolumn{3}{c}{ Alhagi AFN 25.09.1999 } \\
\hline Zeit & $\begin{array}{c}\text { Leitfähigkeit } \\
{\left[\mathrm{mmol}^{*} \mathrm{~m}^{-2 *} \mathrm{~s}^{-1}\right]}\end{array}$ & $\begin{array}{c}\text { Leitfähigkeit } \\
{\left[\mathrm{mmol}^{*} \mathrm{~g}^{-* *} \mathrm{~s}^{-1}\right]}\end{array}$ & Zeit & $\begin{array}{c}\text { Leitfähigkeit } \\
{\left[\mathrm{mmol}^{*} \mathrm{~m}^{-* *} \mathrm{~s}^{-1}\right]}\end{array}$ & $\begin{array}{l}\text { Leitfähigkeit } \\
{\left[\mathrm{mmol}^{*} \mathrm{~g}^{-2 *} \mathrm{~s}^{-1}\right]}\end{array}$ \\
\hline $8: 14$ & $\mathbf{3 3 . 2 4 7} \pm 3.572$ & $\mathbf{0 . 2 8 7} \pm 0.037$ & $10: 29$ & $\mathbf{2 3 . 9 4 9} \pm 1.683$ & $\mathbf{0 . 1 6 5} \pm 0.015$ \\
$10: 32$ & $33.436 \pm 2.314$ & $\mathbf{0 . 2 8 0} \pm 0.028$ & $11: 32$ & $\mathbf{2 3 . 1 6 5} \pm 1.137$ & $\mathbf{0 . 1 5 8} \pm 0.011$ \\
$11: 42$ & $\mathbf{2 9 . 2 4 5} \pm 1.923$ & $\mathbf{0 . 2 4 1} \pm 0.022$ & $12: 32$ & $\mathbf{2 2 . 9 2 1} \pm 1.164$ & $\mathbf{0 . 1 5 7} \pm 0.012$ \\
$12: 51$ & $\mathbf{2 9 . 8 3 7} \pm 1.751$ & $\mathbf{0 . 2 4 5} \pm 0.019$ & $13: 31$ & $\mathbf{2 1 . 0 8 6} \pm 1.184$ & $\mathbf{0 . 1 4 4} \pm 0.012$ \\
$15: 07$ & $\mathbf{2 4 . 6 2 5} \pm 1.184$ & $\mathbf{0 . 2 0 1} \pm 0.014$ & $15: 21$ & $\mathbf{1 7 . 8 0 8} \pm 0.960$ & $\mathbf{0 . 1 2 3} \pm 0.010$ \\
$16: 21$ & $\mathbf{1 9 . 7 0 8} \pm 1.080$ & $\mathbf{0 . 1 6 0} \pm 0.011$ & $16: 25$ & $\mathbf{1 5 . 5 3 3} \pm 0.818$ & $\mathbf{0 . 1 0 7} \pm 0.009$ \\
$17: 28$ & $\mathbf{1 7 . 6 8 5} \pm 1.035$ & $\mathbf{0 . 1 4 4} \pm 0.011$ & $17: 35$ & $\mathbf{1 5 . 3 0 1} \pm 0.880$ & $\mathbf{0 . 1 0 6} \pm 0.009$ \\
$18: 34$ & $\mathbf{1 8 . 4 3 3} \pm 0.887$ & $\mathbf{0 . 1 5 1} \pm 0.011$ & $18: 35$ & $\mathbf{1 5 . 3 7 5} \pm 0.724$ & $\mathbf{0 . 1 0 6} \pm 0.009$ \\
\hline \multicolumn{5}{c}{} \\
\hline
\end{tabular}

\begin{tabular}{ccc|ccc}
\hline \multicolumn{3}{c|}{ Alhagi ADN 24.08.1999 } & \multicolumn{3}{c}{ Alhagi ADN 06.10.1999 } \\
\hline Zeit & $\begin{array}{c}\text { Leitfähigkeit } \\
{\left[\mathrm{mmol}^{*} \mathrm{~m}^{-2 *} \mathrm{~s}^{-1}\right]}\end{array}$ & $\begin{array}{c}\text { Leitfähigkeit } \\
{\left[\mathrm{mmol}^{*} \mathrm{~g}^{-2 *} \mathrm{~s}^{-1}\right]}\end{array}$ & Zeit & $\begin{array}{l}\text { Leitfähigkeit } \\
{\left[\mathrm{mmol}^{*} \mathrm{~m}^{-2 *} \mathrm{~s}^{-1}\right]}\end{array}$ & $\begin{array}{l}\text { Leitfähigkeit } \\
{\left[\mathrm{mmol}^{*} \mathrm{~g}^{-2 *} \mathrm{~s}^{-1}\right]}\end{array}$ \\
\hline $10: 44$ & $\mathbf{2 2 . 7 1 2} \pm 1.658$ & $\mathbf{0 . 1 2 8} \pm 0.010$ & $11: 13$ & $\mathbf{1 6 . 9 1 6} \pm 1.150$ & $\mathbf{0 . 0 7 5} \pm 0.006$ \\
$11: 51$ & $\mathbf{2 3 . 2 2 0} \pm 1.612$ & $\mathbf{0 . 1 3 1} \pm 0.010$ & $12: 15$ & $\mathbf{1 8 . 1 7 8} \pm 1.180$ & $\mathbf{0 . 0 8 0} \pm 0.006$ \\
$12: 52$ & $\mathbf{1 7 . 7 3 0} \pm 1.546$ & $\mathbf{0 . 1 0 0} \pm 0.009$ & $13: 16$ & $\mathbf{1 7 . 8 9 8} \pm 1.145$ & $\mathbf{0 . 0 7 9} \pm 0.005$ \\
$15: 09$ & $\mathbf{1 3 . 2 3 1} \pm 0.876$ & $\mathbf{0 . 0 7 4} \pm 0.005$ & $14: 58$ & $\mathbf{1 5 . 5 9 8} \pm 1.016$ & $\mathbf{0 . 0 6 9} \pm 0.005$ \\
$16: 37$ & $\mathbf{8 . 4 0 1} \pm 0.363$ & $\mathbf{0 . 0 4 7} \pm 0.003$ & $16: 10$ & $\mathbf{1 4 . 1 7 4} \pm 1.124$ & $\mathbf{0 . 0 6 2} \pm 0.005$ \\
$17: 50$ & $\mathbf{1 1 . 8 1 8} \pm 0.575$ & $\mathbf{0 . 0 6 6} \pm 0.003$ & $17: 09$ & $\mathbf{1 3 . 5 8 8} \pm 0.866$ & $\mathbf{0 . 0 5 9} \pm 0.004$ \\
$18: 48$ & $\mathbf{1 2 . 2 9 8} \pm 0.659$ & $\mathbf{0 . 0 6 9} \pm 0.004$ & $18: 07$ & $\mathbf{1 6 . 0 1 3} \pm 1.059$ & $\mathbf{0 . 0 7 0} \pm 0.005$ \\
\hline
\end{tabular}


Tab. 15: Transpiration von Calligonum caput-medusae 1999.

Mittelwerte aus 18 Messungen (6 Pflanzen mit je 3 Parallelen) mit Standardfehler (se). Die Flächenkürzel bedeuten: C Calligonum caput-medusae, $\mathbf{F}$ bewässert (flooded), D unbewässert (dry), $\mathbf{N}$ nicht genutzt.

\begin{tabular}{ccc|ccc}
\hline \multicolumn{3}{c|}{ Calligonum CFN 11.06.1999 } & \multicolumn{3}{c}{ Calligonum CFN 18.07.1999 } \\
\hline Zeit & $\begin{array}{c}\text { Transpiration } \\
{\left[\mathrm{mmol}^{*} \mathrm{~m}^{-2 *} \mathrm{~s}^{-1}\right]}\end{array}$ & $\begin{array}{c}\text { Transpiration } \\
{\left[\mu \mathrm{mol}^{*} \mathrm{~g}^{-2 *} \mathrm{~s}^{-1}\right]}\end{array}$ & Zeit & $\begin{array}{l}\text { Transpiration } \\
{\left[\mathrm{mmol}^{*} \mathrm{~m}^{-2 *} \mathrm{~s}^{-1}\right]}\end{array}$ & $\begin{array}{l}\text { Transpiration } \\
{\left[\mu \mathrm{mol}^{*} \mathrm{~g}^{-2 *} \mathrm{~s}^{-1}\right]}\end{array}$ \\
\hline $7: 37$ & $\mathbf{0 . 1 3 3} \pm 0.011$ & $\mathbf{1 . 3 2 5} \pm 0.111$ & $7: 25$ & $\mathbf{0 . 2 9 7} \pm 0.010$ & $\mathbf{2 . 5 8 6} \pm 0.102$ \\
$8: 32$ & $\mathbf{0 . 0 4 2} \pm 0.008$ & $\mathbf{0 . 4 0 9} \pm 0.073$ & $8: 17$ & $\mathbf{0 . 1 0 8} \pm 0.004$ & $\mathbf{0 . 9 5 1} \pm 0.047$ \\
$10: 22$ & $\mathbf{0 . 3 2 7} \pm 0.028$ & $\mathbf{3 . 2 7 6} \pm 0.302$ & $10: 38$ & $\mathbf{0 . 2 6 1} \pm 0.025$ & $\mathbf{2 . 3 5 0} \pm 0.274$ \\
$11: 24$ & $\mathbf{0 . 3 9 6} \pm 0.022$ & $\mathbf{3 . 9 4 1} \pm 0.245$ & $12: 19$ & $\mathbf{0 . 5 3 2} \pm 0.029$ & $\mathbf{4 . 6 7 5} \pm 0.326$ \\
$12: 24$ & $\mathbf{0 . 5 8 5} \pm 0.028$ & $\mathbf{5 . 8 1 5} \pm 0.321$ & $14: 07$ & $\mathbf{0 . 4 7 3} \pm 0.046$ & $\mathbf{1 . 4 4 0} \pm 0.469$ \\
$13: 22$ & $\mathbf{0 . 6 5 9} \pm 0.028$ & $\mathbf{6 . 5 3 3} \pm 0.313$ & $16: 27$ & $\mathbf{0 . 2 5 6} \pm 0.006$ & $\mathbf{2 . 2 4 0} \pm 0.070$ \\
$14: 39$ & $\mathbf{0 . 6 2 8} \pm 0.031$ & $\mathbf{6 . 2 1 8} \pm 0.339$ & $18: 22$ & $\mathbf{0 . 5 7 8} \pm 0.021$ & $\mathbf{5 . 0 6 4} \pm 0.233$ \\
$15: 43$ & $\mathbf{0 . 5 2 4} \pm 0.029$ & $\mathbf{5 . 1 5 9} \pm 0.271$ & $19: 34$ & $\mathbf{0 . 4 4 7} \pm 0.014$ & $\mathbf{3 . 9 1 4} \pm 0.153$ \\
$17: 24$ & $\mathbf{0 . 3 6 1} \pm 0.040$ & $\mathbf{3 . 6 5 1} \pm 0.428$ & $20: 27$ & $\mathbf{0 . 3 3 5} \pm 0.011$ & $\mathbf{2 . 9 1 9} \pm 0.109$ \\
$19: 19$ & $\mathbf{0 . 2 5 6} \pm 0.022$ & $\mathbf{2 . 5 1 9} \pm 0.210$ & & & \\
\hline
\end{tabular}

\begin{tabular}{ccc|ccc}
\hline \multicolumn{3}{c|}{ Calligonum CFN 15.08.1999 } & \multicolumn{3}{c}{ Calligonum CFN 23.09.1999 } \\
\hline Zeit & $\begin{array}{c}\text { Transpiration } \\
{\left[\mathrm{mmol}^{*} \mathrm{~m}^{-2 *} \mathrm{~s}^{-1}\right]}\end{array}$ & $\begin{array}{c}\text { Transpiration } \\
{\left[\mu \mathrm{mol}^{*} \mathrm{~g}^{-2 *} \mathrm{~s}^{-1}\right]}\end{array}$ & Zeit & $\begin{array}{l}\text { Transpiration } \\
{\left[\mathrm{mmol}^{*} \mathrm{~m}^{-2 *} \mathrm{~s}^{-1}\right]}\end{array}$ & $\begin{array}{l}\text { Transpiration } \\
{\left[\mu \mathrm{mol}^{*} \mathrm{~g}^{-2 *} \mathrm{~s}^{-1}\right]}\end{array}$ \\
\hline $10: 38$ & $\mathbf{0 . 2 7 6} \pm 0.041$ & $\mathbf{2 . 9 7 6} \pm 0.440$ & $8: 40$ & $\mathbf{0 . 3 0 9} \pm 0.022$ & $\mathbf{1 . 1 8 5} \pm 0.145$ \\
$11: 40$ & $\mathbf{0 . 3 6 3} \pm 0.060$ & $\mathbf{3 . 9 0 9} \pm 0.650$ & $10: 38$ & $\mathbf{0 . 4 6 6} \pm 0.038$ & $\mathbf{1 . 7 5 6} \pm 0.203$ \\
$12: 40$ & $\mathbf{0 . 3 9 6} \pm 0.059$ & $\mathbf{4 . 2 6 3} \pm 0.635$ & $11: 44$ & $\mathbf{0 . 7 3 6} \pm 0.043$ & $\mathbf{2 . 7 6 2} \pm 0.286$ \\
$13: 43$ & $\mathbf{0 . 4 7 7} \pm 0.078$ & $\mathbf{5 . 1 3 6} \pm 0.842$ & $12: 44$ & $\mathbf{0 . 8 8 5} \pm 0.061$ & $\mathbf{3 . 3 6 0} \pm 0.412$ \\
$15: 21$ & $\mathbf{0 . 4 9 2} \pm 0.083$ & $\mathbf{5 . 3 0 6} \pm 0.892$ & $13: 39$ & $\mathbf{1 . 0 0 4} \pm 0.049$ & $\mathbf{3 . 7 1 9} \pm 0.348$ \\
$16: 33$ & $\mathbf{0 . 4 2 8} \pm 0.069$ & $\mathbf{4 . 6 1 5} \pm 0.745$ & $15: 16$ & $\mathbf{1 . 0 0 6} \pm 0.047$ & $\mathbf{3 . 7 0 8} \pm 0.328$ \\
$17: 46$ & $\mathbf{0 . 3 8 1} \pm 0.058$ & $\mathbf{4 . 1 0 8} \pm 0.630$ & $16: 21$ & $\mathbf{0 . 9 9 5} \pm 0.047$ & $\mathbf{3 . 6 7 0} \pm 0.353$ \\
$19: 01$ & $\mathbf{0 . 2 2 9} \pm 0.027$ & $\mathbf{2 . 4 6 6} \pm 0.289$ & $17: 27$ & $\mathbf{0 . 8 3 3} \pm 0.039$ & $\mathbf{3 . 0 5 9} \pm 0.266$ \\
& & & $18: 36$ & $\mathbf{0 . 6 7 7} \pm 0.031$ & $\mathbf{2 . 4 5 8} \pm 0.186$ \\
\hline
\end{tabular}

\begin{tabular}{ccc|ccc}
\hline \multicolumn{2}{c|}{ Calligonum CDN 28.08.1999 } & \multicolumn{3}{c}{ Calligonum CDN 29.09.1999 } \\
\hline Zeit & $\begin{array}{c}\text { Transpiration } \\
{\left[\mathrm{mmol}^{*} \mathrm{~m}^{-2 *} \mathrm{~s}^{-1}\right]}\end{array}$ & $\begin{array}{c}\text { Transpiration } \\
{\left[\mu \mathrm{mol}^{*} \mathrm{~g}^{-2 *} \mathrm{~s}^{-1}\right]}\end{array}$ & Zeit & $\begin{array}{l}\text { Transpiration } \\
{\left[\mathrm{mmol}^{*} \mathrm{~m}^{-2 *} \mathrm{~s}^{-1}\right]}\end{array}$ & $\begin{array}{l}\text { Transpiration } \\
{\left[\mu \mathrm{mol}^{*} \mathrm{~g}^{-2 *} \mathrm{~s}^{-1}\right]}\end{array}$ \\
\hline $10: 42$ & $\mathbf{0 . 1 0 8} \pm 0.006$ & $\mathbf{1 . 1 6 7} \pm 0.081$ & $10: 39$ & $\mathbf{0 . 2 2 0} \pm 0.008$ & $\mathbf{2 . 0 7 5} \pm 0.128$ \\
$11: 59$ & $\mathbf{0 . 2 5 9} \pm 0.014$ & $\mathbf{2 . 8 6 4} \pm 0.265$ & $11: 41$ & $\mathbf{0 . 2 7 7} \pm 0.009$ & $\mathbf{2 . 6 0 2} \pm 0.140$ \\
$13: 05$ & $\mathbf{0 . 3 3 2} \pm 0.017$ & $\mathbf{3 . 6 8 4} \pm 0.358$ & $12: 39$ & $\mathbf{0 . 3 3 6} \pm 0.013$ & $\mathbf{3 . 1 6 3} \pm 0.189$ \\
$15: 25$ & $\mathbf{0 . 3 5 4} \pm 0.019$ & $\mathbf{3 . 9 4 5} \pm 0.398$ & $13: 39$ & $\mathbf{0 . 3 7 7} \pm 0.014$ & $\mathbf{3 . 5 3 9} \pm 0.199$ \\
$16: 45$ & $\mathbf{0 . 3 4 3} \pm 0.019$ & $\mathbf{3 . 8 3 5} \pm 0.404$ & $15: 13$ & $\mathbf{0 . 3 9 4} \pm 0.014$ & $\mathbf{3 . 7 0 1} \pm 0.214$ \\
$17: 55$ & $\mathbf{0 . 3 0 2} \pm 0.018$ & $\mathbf{3 . 4 2 2} \pm 0.402$ & $16: 19$ & $\mathbf{0 . 4 0 8} \pm 0.013$ & $\mathbf{3 . 8 6 2} \pm 0.242$ \\
& & & $17: 18$ & $\mathbf{0 . 3 4 1} \pm 0.011$ & $\mathbf{3 . 2 2 2} \pm 0.201$ \\
& & & $18: 13$ & $\mathbf{0 . 2 9 8} \pm 0.008$ & $\mathbf{2 . 7 9 7} \pm 0.164$ \\
\hline
\end{tabular}


Tab. 16: Stomatäre Leitfähigkeit von Calligonum caput-medusae 1999.

Mittelwerte aus 18 Messungen (6 Pflanzen mit je 3 Parallelen) mit Standardfehler (se). Die Flächenkürzel bedeuten: C Calligonum caput-medusae, $\mathbf{F}$ bewässert (flooded), D unbewässert (dry), $\mathbf{N}$ nicht genutzt.

\begin{tabular}{ccc|ccc}
\hline \multicolumn{3}{c|}{ Calligonum CFN 11.06.1999 } & \multicolumn{3}{c}{ Calligonum CFN 18.07.1999 } \\
\hline Zeit & $\begin{array}{c}\text { Leitfähigkeit } \\
{\left[\mathrm{mmol}^{*} \mathrm{~m}^{-2 *} \mathrm{~s}^{-1}\right]}\end{array}$ & $\begin{array}{c}\text { Leitfähigkeit } \\
{\left[\mathrm{mmol}^{*} \mathrm{~g}^{-2 *} \mathrm{~s}^{-1}\right]}\end{array}$ & Zeit & $\begin{array}{l}\text { Leitfähigkeit } \\
{\left[\mathrm{mmol}^{*} \mathrm{~m}^{-2 *} \mathrm{~s}^{-1}\right]}\end{array}$ & $\begin{array}{l}\text { Leitfähigkeit } \\
{\left[\mathrm{mmol}^{*} \mathrm{~g}^{-2 *} \mathrm{~s}^{-1}\right]}\end{array}$ \\
\hline $7: 37$ & $\mathbf{1 8 . 5 1 7} \pm 1.807$ & $\mathbf{0 . 0 3 5} \pm 0.004$ & $7: 25$ & $\mathbf{3 3 . 8 7 8} \pm 1.934$ & $\mathbf{0 . 2 9 4} \pm 0.015$ \\
$8: 32$ & $\mathbf{6 . 4 0 2} \pm 1.214$ & $\mathbf{0 . 0 1 2} \pm 0.003$ & $8: 17$ & $\mathbf{1 3 . 4 8 3} \pm 0.560$ & $\mathbf{0 . 1 1 8} \pm 0.006$ \\
$10: 22$ & $\mathbf{1 2 . 2 7 5} \pm 0.950$ & $\mathbf{0 . 0 2 3} \pm 0.002$ & $10: 38$ & $\mathbf{1 0 . 6 4 5} \pm 0.997$ & $\mathbf{0 . 0 9 6} \pm 0.010$ \\
$11: 24$ & $\mathbf{1 1 . 8 3 7} \pm 0.658$ & $\mathbf{0 . 0 2 2} \pm 0.002$ & $12: 19$ & $\mathbf{1 0 . 2 2 3} \pm 0.849$ & $\mathbf{0 . 0 9 1} \pm 0.010$ \\
$12: 24$ & $\mathbf{1 5 . 1 9 6} \pm 0.718$ & $\mathbf{0 . 0 2 8} \pm 0.002$ & $14: 07$ & $\mathbf{3 . 0 3 0} \pm 1.005$ & $\mathbf{0 . 0 2 4} \pm 0.008$ \\
$13: 22$ & $\mathbf{1 5 . 2 1 3} \pm 0.781$ & $\mathbf{0 . 0 2 8} \pm 0.002$ & $16: 27$ & $\mathbf{3 . 2 9 7} \pm 0.088$ & $\mathbf{0 . 0 2 9} \pm 0.001$ \\
$14: 39$ & $\mathbf{1 3 . 6 3 1} \pm 0.774$ & $\mathbf{0 . 0 2 5} \pm 0.002$ & $18: 22$ & $\mathbf{8 . 1 9 7} \pm 0.414$ & $\mathbf{0 . 0 7 2} \pm 0.004$ \\
$15: 43$ & $\mathbf{1 0 . 3 3 3} \pm 0.620$ & $\mathbf{0 . 0 1 9} \pm 0.002$ & $19: 34$ & $\mathbf{7 . 6 8 0} \pm 0.277$ & $\mathbf{0 . 0 6 7} \pm 0.003$ \\
$17: 24$ & $\mathbf{6 . 7 6 0} \pm 0.743$ & $\mathbf{0 . 0 1 3} \pm 0.002$ & $20: 27$ & $\mathbf{6 . 4 9 3} \pm 0.219$ & $\mathbf{0 . 0 5 7} \pm 0.002$ \\
$19: 19$ & $\mathbf{5 . 6 1 4} \pm 0.479$ & $\mathbf{0 . 0 1 0} \pm 0.001$ & & & \\
\hline
\end{tabular}

\begin{tabular}{ccc|ccc}
\hline \multicolumn{3}{c|}{ Calligonum CFN 15.08.1999 } & \multicolumn{3}{c}{ Calligonum CFN 23.09.1999 } \\
\hline Zeit & $\begin{array}{c}\text { Leitfähigkeit } \\
{\left[\mathrm{mmol}^{*} \mathrm{~m}^{-2 *} \mathrm{~s}^{-1}\right]}\end{array}$ & $\begin{array}{c}\text { Leitfähigkeit } \\
{\left[\mathrm{mmol}^{*} \mathrm{~g}^{-2 *} \mathrm{~s}^{-1}\right]}\end{array}$ & Zeit & $\begin{array}{l}\text { Leitfähigkeit } \\
{\left[\mathrm{mmol}^{*} \mathrm{~m}^{-2 *} \mathrm{~s}^{-1}\right]}\end{array}$ & $\begin{array}{c}\text { Leitfähigkeit } \\
{\left[\mathrm{mmol}^{*} \mathrm{~g}^{-2 *} \mathrm{~s}^{-1}\right]}\end{array}$ \\
\hline $10: 38$ & $\mathbf{1 2 . 1 8 1} \pm 1.914$ & $\mathbf{0 . 1 3 1} \pm 0.021$ & $8: 40$ & $\mathbf{6 4 . 3 4 2} \pm 6.196$ & $\mathbf{0 . 2 4 6} \pm 0.034$ \\
$11: 40$ & $\mathbf{1 2 . 3 8 1} \pm 2.090$ & $\mathbf{0 . 1 3 3} \pm 0.023$ & $10: 38$ & $\mathbf{3 4 . 4 5 5} \pm 1.806$ & $\mathbf{0 . 1 2 5} \pm 0.010$ \\
$12: 40$ & $\mathbf{1 2 . 0 6 2} \pm 2.107$ & $\mathbf{0 . 1 3 0} \pm 0.023$ & $11: 44$ & $\mathbf{2 6 . 8 5 9} \pm 1.619$ & $\mathbf{0 . 0 9 9} \pm 0.009$ \\
$13: 43$ & $\mathbf{1 2 . 5 4 6} \pm 1.976$ & $\mathbf{0 . 1 3 5} \pm 0.021$ & $12: 44$ & $\mathbf{2 4 . 7 0 3} \pm 1.700$ & $\mathbf{0 . 0 9 2} \pm 0.009$ \\
$15: 21$ & $\mathbf{1 0 . 8 2 0} \pm 1.770$ & $\mathbf{0 . 1 1 7} \pm 0.019$ & $13: 39$ & $\mathbf{2 3 . 8 9 9} \pm 1.399$ & $\mathbf{0 . 0 8 7} \pm 0.008$ \\
$16: 33$ & $\mathbf{9 . 2 4 2} \pm 1.535$ & $\mathbf{0 . 1 0 0} \pm 0.017$ & $15: 16$ & $\mathbf{2 1 . 6 0 7} \pm 1.257$ & $\mathbf{0 . 0 8 0} \pm 0.008$ \\
$17: 46$ & $\mathbf{8 . 6 6 7} \pm 1.386$ & $\mathbf{0 . 0 9 3} \pm 0.015$ & $16: 21$ & $\mathbf{2 2 . 5 4 4} \pm 1.365$ & $\mathbf{0 . 0 8 3} \pm 0.009$ \\
$19: 01$ & $\mathbf{5 . 6 2 7} \pm 0.687$ & $\mathbf{0 . 0 6 1} \pm 0.007$ & $17: 27$ & $\mathbf{1 9 . 1 8 6} \pm 1.187$ & $\mathbf{0 . 0 7 2} \pm 0.008$ \\
& & & $18: 36$ & $\mathbf{1 7 . 4 2 9} \pm 0.914$ & $\mathbf{0 . 0 6 4} \pm 0.006$ \\
\hline
\end{tabular}

\begin{tabular}{ccc|ccc}
\hline \multicolumn{3}{c|}{ Calligonum CDN 28.08.1999 } & \multicolumn{3}{c}{ Calligonum CDN 29.09.1999 } \\
\hline Zeit & $\begin{array}{c}\text { Leitfähigkeit } \\
{\left[\mathrm{mmol}^{*} \mathrm{~m}^{-2 *} \mathrm{~s}^{-1}\right]}\end{array}$ & $\begin{array}{c}\text { Leitfähigkeit } \\
{\left[\mathrm{mmol}^{*} \mathrm{~g}^{-2 *} \mathrm{~s}^{-1}\right]}\end{array}$ & Zeit & $\begin{array}{l}\text { Leitfähigkeit } \\
{\left[\mathrm{mmol}^{*} \mathrm{~m}^{-2 *} \mathrm{~s}^{-1}\right]}\end{array}$ & $\begin{array}{l}\text { Leitfähigkeit } \\
{\left[\mathrm{mmol}^{*} \mathrm{~g}^{-2 *} \mathrm{~s}^{-1}\right]}\end{array}$ \\
\hline $10: 42$ & $\mathbf{7 . 3 8 1} \pm 0.418$ & $\mathbf{0 . 0 8 0} \pm 0.006$ & $10: 39$ & $\mathbf{1 8 . 6 7 1} \pm 1.308$ & $\mathbf{0 . 1 7 4} \pm 0.014$ \\
$11: 59$ & $\mathbf{9 . 2 7 0} \pm 0.572$ & $\mathbf{0 . 1 0 1} \pm 0.009$ & $11: 41$ & $\mathbf{1 5 . 8 3 9} \pm 0.774$ & $\mathbf{0 . 1 4 7} \pm 0.008$ \\
$13: 05$ & $\mathbf{7 . 7 8 6} \pm 0.387$ & $\mathbf{0 . 0 8 5} \pm 0.006$ & $12: 39$ & $\mathbf{1 4 . 8 1 1} \pm 0.656$ & $\mathbf{0 . 1 3 8} \pm 0.007$ \\
$15: 25$ & $\mathbf{6 . 8 9 9} \pm 0.367$ & $\mathbf{0 . 0 7 5} \pm 0.006$ & $13: 39$ & $\mathbf{1 3 . 7 4 7} \pm 0.689$ & $\mathbf{0 . 1 2 7} \pm 0.006$ \\
$16: 45$ & $\mathbf{6 . 3 6 0} \pm 0.367$ & $\mathbf{0 . 0 7 1} \pm 0.007$ & $15: 13$ & $\mathbf{1 3 . 1 8 6} \pm 0.529$ & $\mathbf{0 . 1 2 3} \pm 0.006$ \\
$17: 55$ & $\mathbf{6 . 3 0 6} \pm 0.366$ & $\mathbf{0 . 0 7 1} \pm 0.008$ & $16: 19$ & $\mathbf{1 3 . 5 4 9} \pm 0.403$ & $\mathbf{0 . 1 2 7} \pm 0.006$ \\
& & & $17: 18$ & $\mathbf{1 1 . 3 6 3} \pm 0.509$ & $\mathbf{0 . 1 0 6} \pm 0.006$ \\
& & & $18: 13$ & $\mathbf{1 1 . 2 7 8} \pm 0.414$ & $\mathbf{0 . 1 0 5} \pm 0.005$ \\
\hline
\end{tabular}


Tab. 17: Transpiration von Tamarix ramosissima 1999.

Mittelwerte aus 18 Messungen (6 Pflanzen mit je 3 Parallelen) mit Standardfehler (se). Die Flächenkürzel bedeuten: T Tamarix ramosissima, F bewässert (flooded), D unbewässert (dry), $\mathbf{N}$ nicht genutzt.

\begin{tabular}{ccc|ccc}
\hline \multicolumn{3}{c|}{ Tamarix TFN 12.05.1999 } & \multicolumn{3}{c}{ Tamarix TFN 11.06.1999 } \\
\hline Zeit & $\begin{array}{c}\text { Transpiration } \\
{\left[\mathrm{mmol}^{*} \mathrm{~m}^{-2 *} \mathrm{~s}^{-1}\right]}\end{array}$ & $\begin{array}{c}\text { Transpiration } \\
{\left[\mu \mathrm{mol}^{*} \mathrm{~g}^{-2 *} \mathrm{~s}^{-1}\right]}\end{array}$ & Zeit & $\begin{array}{c}\text { Transpiration } \\
{\left[\mathrm{mmol}^{*} \mathrm{~m}^{-2 *} \mathrm{~s}^{-1}\right]}\end{array}$ & $\begin{array}{l}\text { Transpiration } \\
{\left[\mu \mathrm{mol}^{*} \mathrm{~g}^{-2 *} \mathrm{~s}^{-1}\right]}\end{array}$ \\
\hline $7: 49$ & $\mathbf{1 . 7 6 8} \pm 0.156$ & $\mathbf{9 . 9 7 5} \pm 0.664$ & $8: 09$ & $\mathbf{0 . 2 9 2} \pm 0.022$ & $\mathbf{1 . 8 3 2} \pm 0.160$ \\
9:27 & $\mathbf{2 . 7 5 8} \pm 0.367$ & $\mathbf{1 6 . 1 0 8} \pm 2.200$ & $9: 17$ & $\mathbf{0 . 3 6 0} \pm 0.032$ & $\mathbf{2 . 3 0 7} \pm 0.236$ \\
$10: 33$ & $\mathbf{5 . 7 6 7} \pm 0.544$ & $\mathbf{3 3 . 4 5 0} \pm 3.292$ & $11: 00$ & $\mathbf{0 . 5 4 5} \pm 0.052$ & $\mathbf{3 . 4 7 2} \pm 0.374$ \\
$11: 40$ & $\mathbf{4 . 0 5 1} \pm 0.368$ & $\mathbf{2 3 . 0 7 3} \pm 1.673$ & $12: 10$ & $\mathbf{0 . 7 0 6} \pm 0.062$ & $\mathbf{4 . 4 8 6} \pm 0.442$ \\
$12: 36$ & $\mathbf{6 . 0 3 3} \pm 0.338$ & $\mathbf{3 4 . 8 5 2} \pm 2.084$ & $13: 17$ & $\mathbf{1 . 0 4 0} \pm 0.072$ & $\mathbf{6 . 4 5 9} \pm 0.489$ \\
$15: 02$ & $\mathbf{4 . 5 4 3} \pm 0.398$ & $\mathbf{2 5 . 9 0 2} \pm 1.900$ & $15: 04$ & $\mathbf{0 . 8 2 1} \pm 0.048$ & $\mathbf{5 . 1 0 5} \pm 0.323$ \\
$16: 45$ & $\mathbf{2 . 9 0 8} \pm 0.245$ & $\mathbf{1 6 . 5 0 9} \pm 1.135$ & & & \\
$18: 19$ & $\mathbf{2 . 0 6 2} \pm 0.188$ & $\mathbf{1 1 . 8 2 3} \pm 0.858$ & & & \\
$19: 29$ & $\mathbf{1 . 9 8 5} \pm 0.153$ & $\mathbf{1 1 . 2 9 2} \pm 0.690$ & & & \\
\hline
\end{tabular}

\begin{tabular}{ccc|ccc}
\hline \multicolumn{3}{c|}{ Tamarix TFN 12.07.1999 } & \multicolumn{3}{c}{ Tamarix TFN 21.08.1999 } \\
\hline Zeit & $\begin{array}{c}\text { Transpiration } \\
{\left[\mathrm{mmol}^{*} \mathrm{~m}^{-2 *} \mathrm{~s}^{-1}\right]}\end{array}$ & $\begin{array}{c}\text { Transpiration } \\
{\left[\mu \mathrm{mol}^{*} \mathrm{~g}^{-2 *} \mathrm{~s}^{-1}\right]}\end{array}$ & Zeit & $\begin{array}{c}\text { Transpiration } \\
{\left[\mathrm{mmol}^{*} \mathrm{~m}^{-2 *} \mathrm{~s}^{-1}\right]}\end{array}$ & $\begin{array}{l}\text { Transpiration } \\
{\left[\mu \mathrm{mol}^{*} \mathrm{~g}^{-2 *} \mathrm{~s}^{-1}\right]}\end{array}$ \\
\hline $8: 45$ & $\mathbf{0 . 2 0 8} \pm 0.017$ & $\mathbf{1 . 4 3 5} \pm 0.110$ & $8: 41$ & $\mathbf{0 . 1 5 0} \pm 0.011$ & $\mathbf{1 . 0 4 9} \pm 0.080$ \\
$10: 42$ & $\mathbf{0 . 0 5 4} \pm 0.030$ & $\mathbf{0 . 3 4 9} \pm 0.189$ & $10: 01$ & $\mathbf{0 . 2 9 0} \pm 0.030$ & $\mathbf{2 . 0 2 4} \pm 0.216$ \\
$11: 55$ & $\mathbf{0 . 2 2 0} \pm 0.024$ & $\mathbf{1 . 5 0 3} \pm 0.146$ & $11: 08$ & $\mathbf{0 . 3 8 8} \pm 0.033$ & $\mathbf{2 . 7 0 6} \pm 0.237$ \\
$13: 46$ & $\mathbf{0 . 4 2 4} \pm 0.039$ & $\mathbf{2 . 9 1 7} \pm 0.248$ & $12: 21$ & $\mathbf{0 . 6 2 6} \pm 0.051$ & $\mathbf{4 . 3 6 5} \pm 0.365$ \\
$15: 44$ & $\mathbf{0 . 4 0 1} \pm 0.029$ & $\mathbf{2 . 7 0 0} \pm 0.167$ & $13: 32$ & $\mathbf{0 . 8 3 2} \pm 0.068$ & $\mathbf{5 . 8 0 5} \pm 0.490$ \\
$17: 01$ & $\mathbf{0 . 2 6 1} \pm 0.020$ & $\mathbf{1 . 7 5 5} \pm 0.107$ & $15: 41$ & $\mathbf{0 . 8 3 7} \pm 0.068$ & $\mathbf{5 . 8 3 7} \pm 0.489$ \\
& & & $17: 02$ & $\mathbf{0 . 7 5 5} \pm 0.057$ & $\mathbf{5 . 2 6 9} \pm 0.408$ \\
& & & $18: 12$ & $\mathbf{0 . 5 9 1} \pm 0.046$ & $\mathbf{4 . 1 2 0} \pm 0.330$ \\
& & & $19: 01$ & $\mathbf{0 . 5 2 6} \pm 0.048$ & $\mathbf{3 . 6 2 8} \pm 0.360$ \\
\hline
\end{tabular}

\begin{tabular}{ccc}
\hline \multicolumn{3}{c}{ Tamarix TFN 27.09.1999 } \\
\hline Zeit & $\begin{array}{c}\text { Transpiration } \\
{\left[\mathrm{mmol}^{*} \mathrm{~m}^{-2 *} \mathrm{~s}^{-1}\right]}\end{array}$ & $\begin{array}{l}\text { Transpiration } \\
{\left[\mu \mathrm{mol}^{*} \mathrm{~g}^{-2 *} \mathrm{~s}^{-1}\right]}\end{array}$ \\
\hline 8:50 & $\mathbf{0 . 0 8 5} \pm 0.007$ & $\mathbf{0 . 5 5 6} \pm 0.062$ \\
$10: 30$ & $\mathbf{0 . 1 6 8} \pm 0.011$ & $\mathbf{1 . 0 9 2} \pm 0.094$ \\
$11: 37$ & $\mathbf{0 . 2 6 5} \pm 0.016$ & $\mathbf{1 . 7 2 4} \pm 0.146$ \\
$12: 37$ & $\mathbf{0 . 3 8 6} \pm 0.017$ & $\mathbf{2 . 4 8 7} \pm 0.161$ \\
$13: 38$ & $\mathbf{0 . 4 5 0} \pm 0.017$ & $\mathbf{2 . 9 0 2} \pm 0.172$ \\
$15: 23$ & $\mathbf{0 . 4 5 3} \pm 0.017$ & $\mathbf{2 . 9 1 7} \pm 0.174$ \\
$16: 31$ & $\mathbf{0 . 4 0 9} \pm 0.017$ & $\mathbf{2 . 6 4 4} \pm 0.173$ \\
$17: 39$ & $\mathbf{0 . 3 7 2} \pm 0.016$ & $\mathbf{2 . 3 9 3} \pm 0.147$ \\
$18: 42$ & $\mathbf{0 . 3 1 1} \pm 0.012$ & $\mathbf{2 . 0 0 5} \pm 0.121$ \\
\hline
\end{tabular}

\begin{tabular}{ccc|ccc}
\hline \multicolumn{3}{c|}{ Tamarix TDN 04.09.1999 } & \multicolumn{3}{c}{ Tamarix TDN 04.10.1999 } \\
\hline Zeit & $\begin{array}{c}\text { Transpiration } \\
{\left[\mathrm{mmol}^{*} \mathrm{~m}^{-2 *} \mathrm{~s}^{-1}\right]}\end{array}$ & $\begin{array}{c}\text { Transpiration } \\
{\left[\mu \mathrm{mol}^{*} \mathrm{~g}^{-2 *} \mathrm{~s}^{-1}\right]}\end{array}$ & $\begin{array}{l}\text { Zeit } \\
\text { Transpiration } \\
{\left[\mathrm{mmol}^{*} \mathrm{~m}^{-2 *} \mathrm{~s}^{-1}\right]}\end{array}$ & $\begin{array}{c}\text { Transpiration } \\
{\left[\mu \mathrm{mol}^{*} \mathrm{~g}^{-2 *} \mathrm{~s}^{-1}\right]}\end{array}$ \\
\hline $12: 02$ & $\mathbf{0 . 3 2 3} \pm 0.035$ & $\mathbf{1 . 8 8 6} \pm 0.216$ & $11: 22$ & $\mathbf{0 . 1 6 0} \pm 0.011$ & $\mathbf{0 . 7 4 9} \pm 0.044$ \\
$13: 01$ & $\mathbf{0 . 4 6 0} \pm 0.027$ & $\mathbf{2 . 6 1 5} \pm 0.199$ & $12: 21$ & $\mathbf{0 . 2 2 1} \pm 0.015$ & $\mathbf{1 . 0 2 7} \pm 0.047$ \\
$13: 56$ & $\mathbf{0 . 4 7 6} \pm 0.033$ & $\mathbf{2 . 6 5 0} \pm 0.184$ & $13: 21$ & $\mathbf{0 . 2 8 0} \pm 0.016$ & $\mathbf{1 . 3 2 3} \pm 0.066$ \\
$15: 44$ & $\mathbf{0 . 4 7 3} \pm 0.032$ & $\mathbf{2 . 6 2 5} \pm 0.176$ & $14: 20$ & $\mathbf{0 . 3 2 5} \pm 0.018$ & $\mathbf{1 . 5 3 3} \pm 0.076$ \\
$16: 51$ & $\mathbf{0 . 4 1 5} \pm 0.024$ & $\mathbf{2 . 3 7 3} \pm 0.178$ & $15: 50$ & $\mathbf{0 . 3 5 3} \pm 0.020$ & $\mathbf{1 . 6 5 7} \pm 0.069$ \\
$17: 56$ & $\mathbf{0 . 3 8 6} \pm 0.027$ & $\mathbf{2 . 1 6 8} \pm 0.157$ & $16: 53$ & $\mathbf{0 . 3 0 9} \pm 0.018$ & $\mathbf{1 . 4 4 2} \pm 0.070$ \\
$18: 55$ & $\mathbf{0 . 2 9 7} \pm 0.015$ & $\mathbf{1 . 7 9 3} \pm 0.153$ & $17: 52$ & $\mathbf{0 . 2 6 8} \pm 0.017$ & $\mathbf{1 . 2 4 6} \pm 0.062$ \\
\hline
\end{tabular}


Tab. 18: Stomatäre Leitfähigkeit von Tamarix ramosissima 1999.

Mittelwerte aus 18 Messungen (6 Pflanzen mit je 3 Parallelen) mit Standardfehler (se). Die Flächenkürzel bedeuten: T Tamarix ramosissima, $\mathbf{F}$ bewässert (flooded), D unbewässert (dry), $\mathbf{N}$ nicht genutzt.

\begin{tabular}{ccc}
\hline \multicolumn{3}{c}{ Tamarix TFN 12.05.1999 } \\
\hline Zeit & $\begin{array}{c}\text { Leitfähigkeit } \\
{\left[\mathrm{mmol}^{*} \mathrm{~m}^{-2 *} \mathrm{~s}^{-1}\right]}\end{array}$ & \multicolumn{1}{l}{$\begin{array}{l}\text { Leitfähigkeit } \\
{\left[\mathrm{mmol}^{*} \mathrm{~g}^{-2 *} \mathrm{~s}^{-1}\right]}\end{array}$} \\
\hline $7: 49$ & $\mathbf{1 4 6 . 6 3} \pm 13.538$ & $\mathbf{0 . 3 3 0} \pm 0.036$ \\
$9: 27$ & $\mathbf{1 0 0 . 7 7} \pm 12.854$ & $\mathbf{0 . 2 3 4} \pm 0.034$ \\
$10: 33$ & $\mathbf{1 3 6 . 9 9} \pm 17.159$ & $\mathbf{0 . 3 1 8} \pm 0.047$ \\
$11: 40$ & $\mathbf{6 8 . 6 3 7} \pm 8.092$ & $\mathbf{0 . 1 5 4} \pm 0.023$ \\
$12: 36$ & $\mathbf{9 3 . 4 2 0} \pm 5.176$ & $\mathbf{0 . 2 0 1} \pm 0.023$ \\
$15: 02$ & $\mathbf{6 3 . 4 7 7} \pm 6.065$ & $\mathbf{0 . 1 3 8} \pm 0.020$ \\
$16: 45$ & $\mathbf{3 7 . 3 6 6} \pm 3.358$ & $\mathbf{0 . 0 7 9} \pm 0.010$ \\
$18: 19$ & $\mathbf{2 9 . 8 1 7} \pm 2.621$ & $\mathbf{0 . 0 6 3} \pm 0.009$ \\
$19: 29$ & $\mathbf{3 2 . 9 8 5} \pm 2.527$ & $\mathbf{0 . 0 6 9} \pm 0.008$ \\
\hline
\end{tabular}

\begin{tabular}{ccc|ccc}
\hline \multicolumn{3}{c|}{ Tamarix TFN 11.06.1999 } & \multicolumn{3}{c}{ Tamarix TFN 12.07.1999 } \\
\hline Zeit & $\begin{array}{c}\text { Leitfähigkeit } \\
{\left[\mathrm{mmol}^{*} \mathrm{~m}^{-2 *} \mathrm{~s}^{-1}\right]}\end{array}$ & $\begin{array}{c}\text { Leitfähigkeit } \\
{\left[\mathrm{mmol}^{*} \mathrm{~g}^{-2 *} \mathrm{~s}^{-1}\right]}\end{array}$ & Zeit & $\begin{array}{l}\text { Leitfähigkeit } \\
{\left[\mathrm{mmol}^{*} \mathrm{~m}^{-2 *} \mathrm{~s}^{-1}\right]}\end{array}$ & $\begin{array}{l}\text { Leitfähigkeit } \\
{\left[\mathrm{mmol}^{*} \mathrm{~g}^{-2 *} \mathrm{~s}^{-1}\right]}\end{array}$ \\
\hline $8: 09$ & $\mathbf{2 7 . 6 1 6} \pm 2.491$ & $\mathbf{0 . 0 5 2} \pm 0.008$ & $8: 45$ & $\mathbf{1 9 . 4 1 1} \pm 1.571$ & $\mathbf{0 . 1 3 4} \pm 0.010$ \\
$9: 17$ & $\mathbf{3 8 . 4 6 9} \pm 3.759$ & $\mathbf{0 . 0 7 3} \pm 0.012$ & $10: 42$ & $\mathbf{4 . 4 0 7} \pm 2.404$ & $\mathbf{0 . 0 2 9} \pm 0.015$ \\
$11: 00$ & $\mathbf{3 8 . 0 7 6} \pm 3.307$ & $\mathbf{0 . 0 7 2} \pm 0.011$ & $11: 55$ & $\mathbf{1 4 . 0 5 8} \pm 1.126$ & $\mathbf{0 . 0 9 6} \pm 0.007$ \\
$12: 10$ & $\mathbf{3 5 . 8 7 6} \pm 3.043$ & $\mathbf{0 . 0 6 7} \pm 0.010$ & $13: 46$ & $\mathbf{1 9 . 5 1 4} \pm 1.971$ & $\mathbf{0 . 1 3 5} \pm 0.013$ \\
$13: 17$ & $\mathbf{4 0 . 9 3 0} \pm 3.044$ & $\mathbf{0 . 0 7 7} \pm 0.011$ & $15: 44$ & $\mathbf{1 8 . 5 2 2} \pm 1.409$ & $\mathbf{0 . 1 2 5} \pm 0.009$ \\
$15: 04$ & $\mathbf{4 0 . 9 3 4} \pm 3.207$ & $\mathbf{0 . 0 7 7} \pm 0.011$ & $17: 01$ & $\mathbf{1 4 . 1 7 9} \pm 1.107$ & $\mathbf{0 . 0 9 5} \pm 0.006$ \\
\hline
\end{tabular}

\begin{tabular}{ccc|ccc}
\hline \multicolumn{3}{c|}{ Tamarix TFN 21.08.1999 } & \multicolumn{3}{c}{ Tamarix TFN 25.09.1999 } \\
\hline Zeit & $\begin{array}{c}\text { Leitfähigkeit } \\
{\left[\mathrm{mmol}^{*} \mathrm{~m}^{-2 *} \mathrm{~s}^{-1}\right]}\end{array}$ & $\begin{array}{c}\text { Leitfähigkeit } \\
{\left[\mathrm{mmol}^{*} \mathrm{~g}^{-2 *} \mathrm{~s}^{-1}\right]}\end{array}$ & Zeit & $\begin{array}{c}\text { Leitfähigkeit } \\
{\left[\mathrm{mmol}^{*} \mathrm{~m}^{-2 *} \mathrm{~s}^{-1}\right]}\end{array}$ & $\begin{array}{c}\text { Leitfähigkeit } \\
{\left[\mathrm{mmol}^{*} \mathrm{~g}^{-2 *} \mathrm{~s}^{-1}\right]}\end{array}$ \\
\hline $8: 41$ & $\mathbf{1 3 . 1 1 4} \pm 1.115$ & $\mathbf{0 . 0 9 1} \pm 0.008$ & $8: 50$ & $\mathbf{1 0 . 1 2 6} \pm 0.939$ & $\mathbf{0 . 0 6 6} \pm 0.008$ \\
$10: 01$ & $\mathbf{1 7 . 3 7 7} \pm 1.785$ & $\mathbf{0 . 1 2 1} \pm 0.013$ & $10: 30$ & $\mathbf{1 5 . 1 4 6} \pm 0.935$ & $\mathbf{0 . 0 9 9} \pm 0.008$ \\
$11: 08$ & $\mathbf{1 7 . 3 8 3} \pm 1.566$ & $\mathbf{0 . 1 2 1} \pm 0.011$ & $11: 37$ & $\mathbf{1 5 . 2 6 2} \pm 0.876$ & $\mathbf{0 . 0 9 9} \pm 0.008$ \\
$12: 21$ & $\mathbf{1 8 . 3 5 3} \pm 1.513$ & $\mathbf{0 . 1 2 8} \pm 0.011$ & $12: 37$ & $\mathbf{1 5 . 3 3 0} \pm 0.711$ & $\mathbf{0 . 0 9 9} \pm 0.006$ \\
$13: 32$ & $\mathbf{1 9 . 3 9 2} \pm 1.697$ & $\mathbf{0 . 1 3 5} \pm 0.012$ & $13: 38$ & $\mathbf{1 4 . 5 1 3} \pm 0.665$ & $\mathbf{0 . 0 9 4} \pm 0.007$ \\
$15: 41$ & $\mathbf{1 6 . 0 5 2} \pm 1.318$ & $\mathbf{0 . 1 1 2} \pm 0.009$ & $15: 23$ & $\mathbf{1 2 . 4 9 5} \pm 0.569$ & $\mathbf{0 . 0 8 1} \pm 0.005$ \\
$17: 02$ & $\mathbf{1 5 . 6 8 4} \pm 1.296$ & $\mathbf{0 . 1 0 9} \pm 0.009$ & $16: 31$ & $\mathbf{1 1 . 7 5 6} \pm 0.547$ & $\mathbf{0 . 0 7 6} \pm 0.005$ \\
$18: 12$ & $\mathbf{1 3 . 3 1 5} \pm 1.029$ & $\mathbf{0 . 0 9 3} \pm 0.007$ & $17: 39$ & $\mathbf{1 1 . 9 7 7} \pm 0.545$ & $\mathbf{0 . 0 7 7} \pm 0.005$ \\
$19: 01$ & $\mathbf{1 1 . 3 0 5} \pm 1.057$ & $\mathbf{0 . 0 7 8} \pm 0.008$ & $18: 42$ & $\mathbf{1 1 . 3 7 5} \pm 0.555$ & $\mathbf{0 . 0 7 4} \pm 0.005$ \\
\hline
\end{tabular}

\begin{tabular}{ccc|ccc}
\hline \multicolumn{3}{c|}{ Tamarix TDN 04.09.1999 } & \multicolumn{3}{c}{ Tamarix TDN 04.10.1999 } \\
\hline Zeit & $\begin{array}{c}\text { Leitfähigkeit } \\
{\left[\mathrm{mmol}^{*} \mathrm{~m}^{-2 *} \mathrm{~s}^{-1}\right]}\end{array}$ & $\begin{array}{c}\text { Leitfähigkeit } \\
{\left[\mathrm{mmol}^{*} \mathrm{~g}^{-2 *} \mathrm{~s}^{-1}\right]}\end{array}$ & $\begin{array}{l}\text { Zeit } \\
\text { Leitfähigkeit } \\
{\left[\mathrm{mmol}^{*} \mathrm{~m}^{-2 *} \mathrm{~s}^{-1}\right]}\end{array}$ & $\begin{array}{l}\text { Leitfähigkeit } \\
{\left[\mathrm{mmol} \mathrm{g}^{-2 *} \mathrm{~s}^{-1}\right]}\end{array}$ \\
\hline $12: 02$ & $\mathbf{1 0 . 5 6 3} \pm 0.946$ & $\mathbf{0 . 0 6 2} \pm 0.006$ & $11: 22$ & $\mathbf{1 2 . 8 1 1} \pm 1.183$ & $\mathbf{0 . 0 5 9} \pm 0.004$ \\
$13: 01$ & $\mathbf{1 1 . 6 1 6} \pm 0.500$ & $\mathbf{0 . 0 6 7} \pm 0.005$ & $12: 21$ & $\mathbf{1 4 . 4 0 6} \pm 1.331$ & $\mathbf{0 . 0 6 7} \pm 0.005$ \\
$13: 56$ & $\mathbf{1 1 . 1 0 4} \pm 0.700$ & $\mathbf{0 . 0 6 2} \pm 0.004$ & $13: 21$ & $\mathbf{1 4 . 0 4 6} \pm 1.212$ & $\mathbf{0 . 0 6 6} \pm 0.005$ \\
$15: 44$ & $\mathbf{1 0 . 0 6 3} \pm 0.611$ & $\mathbf{0 . 0 5 6} \pm 0.004$ & $14: 20$ & $\mathbf{1 3 . 8 2 5} \pm 1.032$ & $\mathbf{0 . 0 6 5} \pm 0.005$ \\
$16: 51$ & $\mathbf{9 . 3 6 8} \pm 0.504$ & $\mathbf{0 . 0 5 4} \pm 0.004$ & $15: 50$ & $\mathbf{1 2 . 3 4 6} \pm 0.842$ & $\mathbf{0 . 0 5 8} \pm 0.003$ \\
$17: 56$ & $\mathbf{9 . 6 9 8} \pm 0.683$ & $\mathbf{0 . 0 5 4} \pm 0.004$ & $16: 53$ & $\mathbf{1 1 . 3 9 5} \pm 0.808$ & $\mathbf{0 . 0 5 3} \pm 0.004$ \\
$18: 55$ & $\mathbf{9 . 0 9 6} \pm 0.474$ & $\mathbf{0 . 0 5 5} \pm 0.005$ & $17: 52$ & $\mathbf{1 1 . 7 0 2} \pm 0.874$ & $\mathbf{0 . 0 5 4} \pm 0.003$ \\
\hline
\end{tabular}


Tab. 19: Transpiration von Populus euphratica 1999.

Mittelwerte aus 18 Messungen (6 Pflanzen mit je 3 Parallelen) mit Standardfehler (se). Die Flächenkürzel bedeuten: P Populus euphratica, F bewässert (flooded), D unbewässert (dry), $\mathbf{N}$ nicht genutzt.

\begin{tabular}{ccc|ccr}
\hline \multicolumn{3}{c|}{ Populus PFN 24.05.1999 } & \multicolumn{3}{c}{ Populus PFN 08.06.1999 } \\
\hline Zeit & $\begin{array}{c}\text { Transpiration } \\
{\left[\mathrm{mmol}^{*} \mathrm{~m}^{-2 *} \mathrm{~s}^{-1}\right]}\end{array}$ & $\begin{array}{c}\text { Transpiration } \\
{\left[\mu \mathrm{mol}^{*} \mathrm{~g}^{-2 *} \mathrm{~s}^{-1}\right]}\end{array}$ & Zeit & $\begin{array}{c}\text { Transpiration } \\
{\left[\mathrm{mmol}^{*} \mathrm{~m}^{-2 *} \mathrm{~s}^{-1}\right]}\end{array}$ & $\begin{array}{l}\text { Transpiration } \\
{\left[\mu \mathrm{mol}^{*} \mathrm{~g}^{-2 *} \mathrm{~s}^{-1}\right]}\end{array}$ \\
\hline $7: 51$ & $\mathbf{0 . 7 5 8} \pm 0.084$ & $\mathbf{6 . 1 9 5} \pm 0.687$ & $7: 41$ & $\mathbf{0 . 2 8 9} \pm 0.031$ & $\mathbf{2 . 0 5 0} \pm 0.223$ \\
$9: 47$ & $\mathbf{2 . 0 0 4} \pm 0.089$ & $\mathbf{1 6 . 3 6 8} \pm 0.727$ & $8: 46$ & $\mathbf{0 . 8 6 1} \pm 0.077$ & $\mathbf{6 . 1 0 7} \pm 0.543$ \\
$10: 54$ & $\mathbf{2 . 3 3 3} \pm 0.121$ & $\mathbf{1 9 . 0 6 0} \pm 0.992$ & $10: 18$ & $\mathbf{2 . 1 3 4} \pm 0.146$ & $\mathbf{1 5 . 1 3 3} \pm 1.033$ \\
$12: 08$ & $\mathbf{2 . 3 0 4} \pm 0.145$ & $\mathbf{1 8 . 8 2 2} \pm 1.182$ & $11: 25$ & $\mathbf{3 . 3 3 7} \pm 0.240$ & $\mathbf{2 3 . 6 6 7} \pm 1.700$ \\
$13: 13$ & $\mathbf{2 . 5 9 0} \pm 0.179$ & $\mathbf{2 1 . 1 6 1} \pm 1.461$ & $12: 27$ & $\mathbf{3 . 5 2 9} \pm 0.240$ & $\mathbf{2 5 . 0 2 7} \pm 1.704$ \\
$14: 53$ & $\mathbf{2 . 7 1 3} \pm 0.208$ & $\mathbf{2 2 . 1 6 9} \pm 1.696$ & $13: 30$ & $\mathbf{3 . 3 1 2} \pm 0.279$ & $\mathbf{2 3 . 4 8 7} \pm 1.979$ \\
$16: 00$ & $\mathbf{2 . 2 9 3} \pm 0.177$ & $\mathbf{1 8 . 7 3 4} \pm 1.447$ & $15: 05$ & $\mathbf{3 . 1 5 5} \pm 0.267$ & $\mathbf{2 2 . 3 7 4} \pm 1.892$ \\
$17: 28$ & $\mathbf{1 . 3 0 9} \pm 0.100$ & $10.694 \pm 0.821$ & $16: 14$ & $\mathbf{3 . 1 4 1} \pm 0.283$ & $\mathbf{2 2 . 2 7 9} \pm 2.007$ \\
$18: 35$ & $\mathbf{1 . 3 8 8} \pm 0.120$ & $\mathbf{1 1 . 3 3 9} \pm 0.983$ & $17: 22$ & $\mathbf{2 . 5 3 7} \pm 0.265$ & $\mathbf{1 7 . 9 9 0} \pm 1.880$ \\
\hline
\end{tabular}

\begin{tabular}{ccc|ccr}
\hline \multicolumn{3}{c|}{ Populus PFN 08.07.1999 } & \multicolumn{3}{c}{ Populus PFN 18.08.1999 } \\
\hline Zeit & $\begin{array}{c}\text { Transpiration } \\
{\left[\mathrm{mmol}^{*} \mathrm{~m}^{-2 *} \mathrm{~s}^{-1}\right]}\end{array}$ & $\begin{array}{c}\text { Transpiration } \\
{\left[\mu \mathrm{mol}^{*} \mathrm{~g}^{-2 *} \mathrm{~s}^{-1}\right]}\end{array}$ & Zeit & $\begin{array}{c}\text { Transpiration } \\
{\left[\mathrm{mmol}^{*} \mathrm{~m}^{-2 *} \mathrm{~s}^{-1}\right]}\end{array}$ & $\begin{array}{l}\text { Transpiration } \\
{\left[\mu \mathrm{mol}^{*} \mathrm{~g}^{-2 *} \mathrm{~s}^{-1}\right]}\end{array}$ \\
\hline $8: 27$ & $\mathbf{1 . 1 1 5} \pm 0.107$ & $\mathbf{7 . 7 4 3} \pm 0.742$ & $8: 34$ & $\mathbf{0 . 5 0 7} \pm 0.069$ & $\mathbf{3 . 2 2 9} \pm 0.439$ \\
$10: 25$ & $\mathbf{2 . 7 4 4} \pm 0.164$ & $\mathbf{1 9 . 0 5 6} \pm 1.139$ & $9: 48$ & $\mathbf{1 . 3 5 9} \pm 0.092$ & $\mathbf{8 . 6 5 6} \pm 0.584$ \\
$12: 35$ & $\mathbf{3 . 3 3 7} \pm 0.240$ & $\mathbf{2 3 . 1 7 5} \pm 1.664$ & $10: 53$ & $\mathbf{2 . 7 0 7} \pm 0.127$ & $\mathbf{1 7 . 2 4 2} \pm 0.808$ \\
$14: 15$ & $\mathbf{3 . 8 5 0} \pm 0.286$ & $\mathbf{2 6 . 7 3 4} \pm 1.984$ & $12: 09$ & $\mathbf{3 . 5 9 5} \pm 0.167$ & $\mathbf{2 2 . 8 9 8} \pm 1.065$ \\
$16: 28$ & $\mathbf{3 . 2 0 2} \pm 0.363$ & $\mathbf{2 2 . 2 3 9} \pm 2.520$ & $13: 16$ & $\mathbf{4 . 5 4 7} \pm 0.207$ & $\mathbf{2 8 . 9 5 9} \pm 1.319$ \\
$17: 54$ & $\mathbf{2 . 6 6 3} \pm 0.331$ & $\mathbf{1 8 . 4 9 1} \pm 2.300$ & $15: 05$ & $\mathbf{5 . 2 5 1} \pm 0.352$ & $\mathbf{3 3 . 4 4 7} \pm 2.241$ \\
$19: 43$ & $\mathbf{1 . 3 3 2} \pm 0.256$ & $\mathbf{9 . 2 5 3} \pm 1.778$ & $16: 16$ & $\mathbf{3 . 7 2 2} \pm 0.327$ & $\mathbf{2 3 . 7 0 4} \pm 2.081$ \\
\hline
\end{tabular}

\begin{tabular}{ccr|rrr}
\hline \multicolumn{3}{c|}{ Populus PFN 14.09.1999 } & \multicolumn{3}{c}{ Populus PDN 31.08.1999 } \\
\hline Zeit & $\begin{array}{c}\text { Transpiration } \\
{\left[\mathrm{mmol}^{*} \mathrm{~m}^{-2 *} \mathrm{~s}^{-1}\right]}\end{array}$ & $\begin{array}{c}\text { Transpiration } \\
{\left[\mu \mathrm{mol}^{*} \mathrm{~g}^{-2 *} \mathrm{~s}^{-1}\right]}\end{array}$ & Zeit & $\begin{array}{c}\text { Transpiration } \\
{\left[\mathrm{mmol}^{*} \mathrm{~m}^{-2 *} \mathrm{~s}^{-1}\right]}\end{array}$ & $\begin{array}{l}\text { Transpiration } \\
{\left[\mu \mathrm{mol}^{*} \mathrm{~g}^{-2 *} \mathrm{~s}^{-1}\right]}\end{array}$ \\
\hline $8: 34$ & $\mathbf{0 . 3 0 3} \pm 0.014$ & $\mathbf{1 . 8 5 4} \pm 0.088$ & $12: 03$ & $\mathbf{2 . 8 1 3} \pm 0.251$ & $\mathbf{1 7 . 5 9 3} \pm 1.570$ \\
$9: 42$ & $\mathbf{0 . 4 9 1} \pm 0.033$ & $\mathbf{2 . 9 9 8} \pm 0.200$ & $13: 14$ & $\mathbf{4 . 2 3 3} \pm 0.257$ & $\mathbf{2 6 . 4 7 2} \pm 1.605$ \\
$11: 16$ & $\mathbf{1 . 1 4 8} \pm 0.083$ & $\mathbf{7 . 0 1 4} \pm 0.504$ & $15: 01$ & $\mathbf{4 . 0 6 7} \pm 0.347$ & $\mathbf{2 5 . 4 3 3} \pm 2.173$ \\
$12: 25$ & $\mathbf{1 . 7 9 5} \pm 0.127$ & $\mathbf{1 0 . 9 6 8} \pm 0.775$ & $17: 13$ & $\mathbf{2 . 0 4 2} \pm 0.166$ & $\mathbf{1 2 . 7 7 1} \pm 1.036$ \\
$13: 36$ & $\mathbf{2 . 0 3 4} \pm 0.135$ & $\mathbf{1 2 . 4 2 2} \pm 0.823$ & & & \\
$15: 16$ & $\mathbf{2 . 4 6 0} \pm 0.174$ & $\mathbf{1 5 . 0 2 9} \pm 1.061$ & & & \\
$16: 41$ & $\mathbf{2 . 4 7 6} \pm 0.164$ & $\mathbf{1 5 . 1 2 7} \pm 1.003$ & & & \\
$18: 29$ & $\mathbf{1 . 4 6 4} \pm 0.114$ & $\mathbf{8 . 9 4 4} \pm 0.699$ & & & \\
\hline
\end{tabular}


Tab. 20: Stomatäre Leitfähigkeit von Populus euphratica 1999.

Mittelwerte aus 18 Messungen (6 Pflanzen mit je 3 Parallelen) mit Standardfehler (se). Die Flächenkürzel bedeuten: P Populus euphratica, $\mathbf{F}$ bewässert (flooded), D unbewässert (dry), N nicht genutzt.

\begin{tabular}{ccc|ccc}
\hline \multicolumn{3}{c|}{ Populus PFN 24.05.1999 } & \multicolumn{3}{c}{ Populus PFN 08.06.1999 } \\
\hline Zeit & $\begin{array}{c}\text { Leitfähigkeit } \\
{\left[\mathrm{mmol}^{*} \mathrm{~m}^{-2 *} \mathrm{~s}^{-1}\right]}\end{array}$ & $\begin{array}{c}\text { Leitfähigkeit } \\
{\left[\mathrm{mmol}^{*} \mathrm{~g}^{-2 *} \mathrm{~s}^{-1}\right]}\end{array}$ & Zeit & $\begin{array}{l}\text { Leitfähigkeit } \\
{\left[\mathrm{mmol}^{*} \mathrm{~m}^{-2 *} \mathrm{~s}^{-1}\right]}\end{array}$ & $\begin{array}{l}\text { Leitfähigkeit } \\
{\left[\mathrm{mmol}^{*} \mathrm{~g}^{-2 *} \mathrm{~s}^{-1}\right]}\end{array}$ \\
\hline $7: 51$ & $\mathbf{5 6 . 9 5 6} \pm 6.674$ & $\mathbf{0 . 4 6 5} \pm 0.055$ & $7: 41$ & $\mathbf{2 6 . 0 4 7} \pm 2.876$ & $\mathbf{0 . 1 8 5} \pm 0.020$ \\
$9: 47$ & $\mathbf{1 3 6 . 5 1 1} \pm 5.630$ & $\mathbf{1 . 1 1 5} \pm 0.046$ & $8: 46$ & $\mathbf{8 2 . 2 9 4} \pm 6.773$ & $\mathbf{0 . 5 8 4} \pm 0.048$ \\
$10: 54$ & $\mathbf{1 0 1 . 6 7 8} \pm 5.992$ & $\mathbf{0 . 8 3 1} \pm 0.049$ & $10: 18$ & $\mathbf{1 3 1 . 9 1 1} \pm 9.028$ & $\mathbf{0 . 9 3 6} \pm 0.064$ \\
$12: 08$ & $\mathbf{8 1 . 4 9 4} \pm 4.384$ & $\mathbf{0 . 6 6 6} \pm 0.036$ & $11: 25$ & $\mathbf{1 4 9 . 7 1 1} \pm 11.73$ & $\mathbf{1 . 0 6 2} \pm 0.083$ \\
$13: 13$ & $\mathbf{8 0 . 6 7 2} \pm 6.064$ & $\mathbf{0 . 6 5 9} \pm 0.050$ & $12: 27$ & $\mathbf{1 0 6 . 2 7 2} \pm 6.577$ & $\mathbf{0 . 7 5 4} \pm 0.047$ \\
$14: 53$ & $\mathbf{7 1 . 4 3 3} \pm 5.651$ & $\mathbf{0 . 5 8 4} \pm 0.046$ & $13: 30$ & $\mathbf{8 3 . 6 8 9} \pm 6.521$ & $\mathbf{0 . 5 9 4} \pm 0.046$ \\
$16: 00$ & $\mathbf{6 0 . 1 2 8} \pm 5.026$ & $\mathbf{0 . 4 9 1} \pm 0.041$ & $15: 05$ & $\mathbf{7 0 . 3 6 7} \pm 6.507$ & $\mathbf{0 . 4 9 9} \pm 0.046$ \\
$17: 28$ & $\mathbf{3 7 . 1 0 0} \pm 3.259$ & $\mathbf{0 . 3 0 3} \pm 0.027$ & $16: 14$ & $\mathbf{6 4 . 0 2 8} \pm 5.470$ & $\mathbf{0 . 4 5 4} \pm 0.039$ \\
$18: 35$ & $\mathbf{4 1 . 5 6 1} \pm 3.904$ & $\mathbf{0 . 3 4 0} \pm 0.032$ & $17: 22$ & $\mathbf{5 3 . 0 3 3} \pm 5.812$ & $\mathbf{0 . 3 7 6} \pm 0.041$ \\
\hline
\end{tabular}

\begin{tabular}{|c|c|c|c|c|c|}
\hline \multicolumn{3}{|c|}{ Populus PFN 08.07.1999 } & \multicolumn{3}{|c|}{ Populus PFN 18.08.1999 } \\
\hline Zeit & $\begin{array}{l}\text { Leitfähigkeit } \\
{\left[\mathrm{mmol}^{*} \mathrm{~m}^{-2 *} \mathrm{~s}^{-1}\right]}\end{array}$ & $\begin{array}{l}\text { Leitfähigkeit } \\
{\left[\mathrm{mmol}^{*} \mathrm{~g}^{-2 *} \mathrm{~s}^{-1}\right]}\end{array}$ & Zeit & $\begin{array}{l}\text { Leitfähigkeit } \\
{\left[\mathrm{mmol}^{*} \mathrm{~m}^{-2 *} \mathrm{~s}^{-1}\right]}\end{array}$ & $\begin{array}{l}\text { Leitfähigkeit } \\
{\left[\mathrm{mmol}^{*} \mathrm{~g}^{-2 *} \mathrm{~s}^{-1}\right]}\end{array}$ \\
\hline $8: 27$ & $98.017 \pm 8.942$ & $0.681 \pm 0.062$ & $8: 34$ & $39.961 \pm 5.494$ & $0.255 \pm 0.035$ \\
\hline $10: 25$ & $165.30 \pm 10.073$ & $1.148 \pm 0.070$ & 9:48 & $113.772 \pm 7.473$ & $0.725 \pm 0.048$ \\
\hline $12: 35$ & $137.356 \pm 11.35$ & $0.954 \pm 0.079$ & $10: 53$ & $164.039 \pm 8.749$ & $1.045 \pm 0.056$ \\
\hline $14: 15$ & $133.061 \pm 9.690$ & $0.924 \pm 0.067$ & $12: 09$ & $169.444 \pm 8.259$ & $1.079 \pm 0.053$ \\
\hline $16: 28$ & $\mathbf{9 0 . 1 8 3} \pm 10.304$ & $\mathbf{0 . 6 2 6} \pm 0.072$ & $13: 16$ & $166.667 \pm 7.034$ & $1.062 \pm 0.045$ \\
\hline $17: 54$ & $80.672 \pm 14.366$ & $0.560 \pm 0.100$ & 15:05 & $147.817 \pm 8.833$ & $0.942 \pm 0.056$ \\
\hline 19:43 & 09 & $0.262 \pm c$ & $16: 16$ & $102.789 \pm 8.765$ & $0.655 \pm 0.056$ \\
\hline
\end{tabular}

\begin{tabular}{ccc|ccc}
\hline \multicolumn{3}{c|}{ Populus PFN } & $\mathbf{1 4 . 0 9 . 1 9 9 9}$ & \multicolumn{3}{c}{ Populus PDN } & 31.08.1999 \\
\hline Zeit & $\begin{array}{c}\text { Leitfähigkeit } \\
{\left[\mathrm{mmol}^{*} \mathrm{~m}^{-2 *} \mathrm{~s}^{-1}\right]}\end{array}$ & $\begin{array}{c}\text { Leitfähigkeit } \\
{\left[\mathrm{mmol}^{*} \mathrm{~g}^{-2 *} \mathrm{~s}^{-1}\right]}\end{array}$ & Zeit & $\begin{array}{l}\text { Leitfähigkeit } \\
{\left[\mathrm{mmol}^{*} \mathrm{~m}^{-2 *} \mathrm{~s}^{-1}\right]}\end{array}$ & $\begin{array}{l}\text { Leitfähigkeit } \\
{\left[\mathrm{mmol}^{*} \mathrm{~g}^{-2 *} \mathrm{~s}^{-1}\right]}\end{array}$ \\
\hline $8: 34$ & $\mathbf{3 0 . 1 1 1} \pm 1.403$ & $\mathbf{0 . 1 8 4} \pm 0.009$ & $12: 03$ & $\mathbf{8 0 . 8 6 7} \pm 6.864$ & $\mathbf{0 . 5 0 6} \pm 0.043$ \\
$9: 42$ & $\mathbf{4 0 . 4 3 9} \pm 2.024$ & $\mathbf{0 . 2 4 7} \pm 0.012$ & $13: 14$ & $\mathbf{8 8 . 0 3 3} \pm 5.022$ & $\mathbf{0 . 5 5 1} \pm 0.031$ \\
$11: 16$ & $\mathbf{4 8 . 5 3 9} \pm 1.572$ & $\mathbf{0 . 2 9 7} \pm 0.010$ & $15: 01$ & $\mathbf{6 7 . 8 0 0} \pm 5.618$ & $\mathbf{0 . 4 2 4} \pm 0.035$ \\
$12: 25$ & $\mathbf{4 9 . 6 5 6} \pm 1.793$ & $\mathbf{0 . 3 0 3} \pm 0.011$ & $17: 13$ & $\mathbf{3 6 . 2 6 7} \pm 3.263$ & $\mathbf{0 . 2 2 7} \pm 0.020$ \\
$13: 36$ & $\mathbf{4 3 . 8 6 1} \pm 2.642$ & $\mathbf{0 . 2 6 8} \pm 0.016$ & & & \\
$15: 16$ & $\mathbf{4 9 . 2 6 1} \pm 2.645$ & $\mathbf{0 . 3 0 1} \pm 0.016$ & & & \\
$16: 41$ & $\mathbf{4 7 . 6 6 7} \pm 3.328$ & $\mathbf{0 . 2 9 1} \pm 0.020$ & & & \\
$18: 29$ & $\mathbf{3 1 . 5 4 4} \pm 2.534$ & $\mathbf{0 . 1 9 3} \pm 0.015$ & & & \\
\hline
\end{tabular}


Tab. 21: Transpiration von Alhagi sparsifolia 2000.

Mittelwerte aus 18 Messungen (6 Pflanzen mit je 3 Parallelen) mit Standardfehler (se). Die Flächenkürzel bedeuten: A Alhagi sparsifolia, F bewässert (flooded), $\mathbf{N}$ nicht genutzt.

\begin{tabular}{ccc|ccc}
\hline \multicolumn{3}{c|}{ Alhagi AFN 22.05.2000 } & \multicolumn{3}{c}{ Alhagi AFN 24.06.2000 } \\
\hline Zeit & $\begin{array}{c}\text { Transpiration } \\
{\left[\mathrm{mmol}^{*} \mathrm{~m}^{-2 *} \mathrm{~s}^{-1}\right]}\end{array}$ & $\begin{array}{c}\text { Transpiration } \\
{\left[\mu \mathrm{mol}^{*} \mathrm{~g}^{-2 *} \mathrm{~s}^{-1}\right]}\end{array}$ & Zeit & $\begin{array}{c}\text { Transpiration } \\
{\left[\mathrm{mmol}^{*} \mathrm{~m}^{-2 *} \mathrm{~s}^{-1}\right]}\end{array}$ & $\begin{array}{l}\text { Transpiration } \\
{\left[\mu \mathrm{mol}^{*} \mathrm{~g}^{-2 *} \mathrm{~s}^{-1}\right]}\end{array}$ \\
\hline $10: 12$ & $\mathbf{1 . 0 4 8} \pm 0.091$ & $\mathbf{9 . 2 5 4} \pm 0.843$ & $10: 20$ & $\mathbf{0 . 5 2 6} \pm 0.042$ & $\mathbf{3 . 1 8 2} \pm 0.316$ \\
$11: 14$ & $\mathbf{1 . 4 0 3} \pm 0.077$ & $\mathbf{1 2 . 3 6 3} \pm 0.700$ & $11: 38$ & $\mathbf{0 . 7 0 1} \pm 0.040$ & $\mathbf{4 . 2 1 5} \pm 0.303$ \\
$12: 42$ & $\mathbf{1 . 7 4 7} \pm 0.098$ & $\mathbf{1 5 . 4 0 2} \pm 0.925$ & $12: 45$ & $\mathbf{0 . 8 3 2} \pm 0.052$ & $\mathbf{5 . 0 0 0} \pm 0.384$ \\
$14: 08$ & $\mathbf{2 . 1 1 2} \pm 0.116$ & $\mathbf{1 8 . 6 2 6} \pm 1.102$ & $14: 57$ & $\mathbf{1 . 0 3 0} \pm 0.071$ & $\mathbf{6 . 2 1 6} \pm 0.527$ \\
$15: 19$ & $\mathbf{2 . 2 2 8} \pm 0.110$ & $\mathbf{1 9 . 6 3 6} \pm 1.048$ & $16: 06$ & $\mathbf{1 . 1 9 8} \pm 0.058$ & $\mathbf{7 . 1 6 0} \pm 0.434$ \\
$16: 29$ & $\mathbf{2 . 0 9 2} \pm 0.099$ & $\mathbf{1 8 . 4 3 3} \pm 0.944$ & $17: 15$ & $\mathbf{1 . 1 9 1} \pm 0.052$ & $\mathbf{7 . 1 0 6} \pm 0.397$ \\
$17: 28$ & $\mathbf{1 . 9 4 5} \pm 0.101$ & $\mathbf{1 7 . 1 5 0} \pm 0.960$ & & & \\
\hline
\end{tabular}

\begin{tabular}{ccr|rrr}
\hline \multicolumn{3}{c|}{ Alhagi AFN 05.08.2000 } & \multicolumn{3}{c}{ Alhagi AFN 16.09.2000 } \\
\hline Zeit & $\begin{array}{c}\text { Transpiration } \\
{\left[\mathrm{mmol}^{*} \mathrm{~m}^{-2 *} \mathrm{~s}^{-1}\right]}\end{array}$ & $\begin{array}{c}\text { Transpiration } \\
{\left[\mu \mathrm{mol}^{*} \mathrm{~g}^{-2 *} \mathrm{~s}^{-1}\right]}\end{array}$ & Zeit & $\begin{array}{c}\text { Transpiration } \\
{\left[\mathrm{mmol}^{*} \mathrm{~m}^{-2 *} \mathrm{~s}^{-1}\right]}\end{array}$ & $\begin{array}{l}\text { Transpiration } \\
{\left[\mu \mathrm{mol}^{*} \mathrm{~g}^{-2 *} \mathrm{~s}^{-1}\right]}\end{array}$ \\
\hline $10: 31$ & $\mathbf{0 . 9 1 8} \pm 0.088$ & $\mathbf{4 . 9 6 2} \pm 0.657$ & $10: 56$ & $\mathbf{0 . 9 8 4} \pm 0.080$ & $\mathbf{4 . 3 6 5} \pm 0.432$ \\
$11: 56$ & $\mathbf{1 . 3 5 7} \pm 0.094$ & $\mathbf{7 . 2 1 1} \pm 0.752$ & $12: 02$ & $\mathbf{1 . 3 0 2} \pm 0.072$ & $\mathbf{5 . 7 4 9} \pm 0.421$ \\
$13: 08$ & $\mathbf{1 . 7 3 5} \pm 0.112$ & $\mathbf{9 . 2 7 0} \pm 0.999$ & $13: 09$ & $\mathbf{1 . 4 9 5} \pm 0.089$ & $\mathbf{6 . 5 9 6} \pm 0.498$ \\
$15: 35$ & $\mathbf{2 . 1 4 4} \pm 0.143$ & $\mathbf{1 1 . 4 2 4} \pm 1.189$ & $14: 42$ & $\mathbf{1 . 6 8 5} \pm 0.099$ & $\mathbf{7 . 4 4 8} \pm 0.580$ \\
$17: 06$ & $\mathbf{2 . 0 4 3} \pm 0.094$ & $\mathbf{1 0 . 6 9 6} \pm 0.851$ & $15: 51$ & $\mathbf{1 . 7 3 5} \pm 0.109$ & $\mathbf{7 . 6 7 2} \pm 0.623$ \\
$18: 17$ & $\mathbf{1 . 7 0 9} \pm 0.085$ & $\mathbf{8 . 9 9 7} \pm 0.788$ & $16: 57$ & $\mathbf{1 . 7 0 7} \pm 0.096$ & $\mathbf{7 . 5 4 0} \pm 0.562$ \\
& & & $18: 04$ & $\mathbf{1 . 5 1 5} \pm 0.081$ & $\mathbf{6 . 6 8 0} \pm 0.475$ \\
& & & $19: 15$ & $\mathbf{1 . 2 8 4} \pm 0.059$ & $\mathbf{5 . 6 2 5} \pm 0.319$ \\
\hline
\end{tabular}

Tab. 22: Stomatäre Leitfähigkeit von Alhagi sparsifolia 2000.

Mittelwerte aus 18 Messungen (6 Pflanzen mit je 3 Parallelen) mit Standardfehler (se). Die Flächenkürzel bedeuten: A Alhagi sparsifolia, $\mathbf{F}$ bewässert (flooded), $\mathbf{N}$ nicht genutzt.

\begin{tabular}{ccc|ccc}
\hline \multicolumn{3}{c|}{ Alhagi AFN 22.05.2000 } & \multicolumn{3}{c}{ Alhagi AFN 24.06.2000 } \\
\hline Zeit & $\begin{array}{c}\text { Leitfähigkeit } \\
{\left[\mathrm{mmol}^{*} \mathrm{~m}^{-2 *} \mathrm{~s}^{-1}\right]}\end{array}$ & $\begin{array}{c}\text { Leitfähigkeit } \\
{\left[\mathrm{mmol}^{*} \mathrm{~g}^{-2 *} \mathrm{~s}^{-1}\right]}\end{array}$ & Zeit & $\begin{array}{c}\text { Leitfähigkeit } \\
{\left[\mathrm{mmol}^{*} \mathrm{~m}^{-2 *} \mathrm{~s}^{-1}\right]}\end{array}$ & $\begin{array}{l}\text { Leitfähigkeit } \\
{\left[\mathrm{mmol}^{*} \mathrm{~g}^{-2 *} \mathrm{~s}^{-1}\right]}\end{array}$ \\
\hline $10: 12$ & $\mathbf{3 4 . 1 1 4} \pm 3.107$ & $\mathbf{0 . 3 0 1} \pm 0.029$ & $10: 20$ & $\mathbf{3 0 . 6 1 3} \pm 2.472$ & $\mathbf{0 . 1 8 4} \pm 0.018$ \\
$11: 14$ & $\mathbf{3 7 . 1 8 4} \pm 2.153$ & $\mathbf{0 . 3 2 7} \pm 0.019$ & $11: 38$ & $\mathbf{2 9 . 1 2 7} \pm 2.217$ & $\mathbf{0 . 1 7 6} \pm 0.016$ \\
$12: 42$ & $\mathbf{3 7 . 9 5 3} \pm 2.449$ & $\mathbf{0 . 3 3 5} \pm 0.023$ & $12: 45$ & $\mathbf{2 8 . 1 3 4} \pm 1.922$ & $\mathbf{0 . 1 6 9} \pm 0.014$ \\
$14: 08$ & $\mathbf{3 8 . 9 7 8} \pm 2.221$ & $\mathbf{0 . 3 4 4} \pm 0.021$ & $14: 57$ & $\mathbf{3 1 . 7 7 9} \pm 2.441$ & $\mathbf{0 . 1 9 2} \pm 0.018$ \\
$15: 19$ & $\mathbf{3 4 . 8 6 0} \pm 2.167$ & $\mathbf{0 . 3 0 7} \pm 0.021$ & $16: 06$ & $\mathbf{2 8 . 8 4 0} \pm 1.255$ & $\mathbf{0 . 1 7 2} \pm 0.009$ \\
$16: 29$ & $\mathbf{3 0 . 6 1 4} \pm 1.595$ & $\mathbf{0 . 2 7 0} \pm 0.015$ & $17: 15$ & $\mathbf{2 6 . 8 5 5} \pm 1.407$ & $\mathbf{0 . 1 6 1} \pm 0.011$ \\
$17: 28$ & $\mathbf{2 8 . 5 7 5} \pm 1.556$ & $\mathbf{0 . 2 5 2} \pm 0.014$ & & & \\
\hline
\end{tabular}

\begin{tabular}{ccc|ccc}
\hline \multicolumn{3}{c|}{ Alhagi AFN 05.08.2000 } & \multicolumn{3}{c}{ Alhagi AFN 16.09.2000 } \\
\hline Zeit & $\begin{array}{c}\text { Leitfähigkeit } \\
{\left[\mathrm{mmol}^{*} \mathrm{~m}^{-2 *} \mathrm{~s}^{-1}\right]}\end{array}$ & $\begin{array}{c}\text { Leitfähigkeit } \\
{\left[\mathrm{mmol}^{*} \mathrm{~g}^{-2 *} \mathrm{~s}^{-1}\right]}\end{array}$ & Zeit & $\begin{array}{l}\text { Leitfähigkeit } \\
{\left[\mathrm{mmol}^{*} \mathrm{~m}^{-2 *} \mathrm{~s}^{-1}\right]}\end{array}$ & $\begin{array}{l}\text { Leitfähigkeit } \\
{\left[\mathrm{mol}^{*} \mathrm{~g}^{-2 *} \mathrm{~s}^{-1}\right]}\end{array}$ \\
\hline $10: 31$ & $\mathbf{3 8 . 8 4 3} \pm 3.049$ & $\mathbf{0 . 2 0 7} \pm 0.023$ & $10: 56$ & $\mathbf{3 9 . 9 9 6} \pm 2.393$ & $\mathbf{0 . 1 7 5} \pm 0.012$ \\
$11: 56$ & $\mathbf{4 0 . 1 2 4} \pm 3.100$ & $\mathbf{0 . 2 1 1} \pm 0.022$ & $12: 02$ & $\mathbf{3 8 . 5 9 9} \pm 1.480$ & $\mathbf{0 . 1 6 9} \pm 0.008$ \\
$13: 08$ & $\mathbf{3 7 . 9 3 2} \pm 2.385$ & $\mathbf{0 . 1 9 8} \pm 0.018$ & $13: 09$ & $\mathbf{3 3 . 7 7 5} \pm 1.630$ & $\mathbf{0 . 1 4 9} \pm 0.009$ \\
$15: 35$ & $\mathbf{3 5 . 9 0 1} \pm 2.196$ & $\mathbf{0 . 1 9 0} \pm 0.018$ & $14: 42$ & $\mathbf{2 8 . 5 7 0} \pm 1.143$ & $\mathbf{0 . 1 2 6} \pm 0.007$ \\
$17: 06$ & $33.149 \pm 1.841$ & $\mathbf{0 . 1 7 2} \pm 0.014$ & $15: 51$ & $\mathbf{2 7 . 2 8 9} \pm 1.750$ & $\mathbf{0 . 1 2 0} \pm 0.009$ \\
$18: 17$ & $\mathbf{3 1 . 8 9 1} \pm 1.988$ & $\mathbf{0 . 1 6 6} \pm 0.013$ & $16: 57$ & $\mathbf{2 8 . 1 6 0} \pm 1.565$ & $\mathbf{0 . 1 2 4} \pm 0.009$ \\
& & & $18: 04$ & $\mathbf{2 9 . 4 7 6} \pm 1.768$ & $\mathbf{0 . 1 3 1} \pm 0.011$ \\
& & & $19: 15$ & $\mathbf{3 0 . 2 7 5} \pm 1.524$ & $\mathbf{0 . 1 3 2} \pm 0.008$ \\
\hline
\end{tabular}


Tab. 23: Transpiration von Calligonum caput-medusae 2000.

Mittelwerte aus 18 Messungen (6 Pflanzen mit je 3 Parallelen) mit Standardfehler (se). Die Flächenkürzel bedeuten: $\mathbf{C}$ Calligonum caput-medusae, $\mathbf{F}$ bewässert (flooded), $\mathbf{N}$ nicht genutzt (not used), U genutzt (used).

\begin{tabular}{ccc|ccr}
\hline \multicolumn{3}{c|}{ Calligonum CFN 17.05.2000 } & \multicolumn{3}{c}{ Calligonum CFU 17.05.2000 } \\
\hline Zeit & $\begin{array}{c}\text { Transpiration } \\
{\left[\mathrm{mmol}^{*} \mathrm{~m}^{-2 *} \mathrm{~s}^{-1}\right]}\end{array}$ & $\begin{array}{c}\text { Transpiration } \\
{\left[\mu \mathrm{mol}^{*} \mathrm{~g}^{-2 *} \mathrm{~s}^{-1}\right]}\end{array}$ & Zeit & $\begin{array}{l}\text { Transpiration } \\
{\left[\mathrm{mmol}^{*} \mathrm{~m}^{-2 *} \mathrm{~s}^{-1}\right]}\end{array}$ & $\begin{array}{l}\text { Transpiration } \\
{\left[\mu \mathrm{mol}^{*} \mathrm{~g}^{-2 *} \mathrm{~s}^{-1}\right]}\end{array}$ \\
\hline $10: 01$ & $\mathbf{0 . 3 5 1} \pm 0.022$ & $\mathbf{4 . 8 1 1} \pm 0.364$ & $10: 17$ & $\mathbf{0 . 4 4 7} \pm 0.024$ & $\mathbf{7 . 3 0 3} \pm 0.386$ \\
$11: 00$ & $\mathbf{0 . 4 7 6} \pm 0.031$ & $\mathbf{6 . 4 9 5} \pm 0.511$ & $11: 16$ & $\mathbf{0 . 6 4 4} \pm 0.030$ & $\mathbf{1 0 . 5 8 0} \pm 0.572$ \\
$12: 01$ & $\mathbf{0 . 6 1 1} \pm 0.034$ & $\mathbf{8 . 3 4 4} \pm 0.585$ & $12: 23$ & $\mathbf{0 . 8 1 5} \pm 0.040$ & $\mathbf{1 3 . 2 7 7} \pm 0.626$ \\
$12: 57$ & $\mathbf{0 . 7 8 7} \pm 0.029$ & $10.778 \pm 0.605$ & $13: 21$ & $\mathbf{0 . 8 3 7} \pm 0.034$ & $\mathbf{1 3 . 7 1 8} \pm 0.635$ \\
$14: 14$ & $\mathbf{0 . 9 3 2} \pm 0.034$ & $\mathbf{1 2 . 7 2 4} \pm 0.670$ & $14: 54$ & $\mathbf{0 . 8 0 8} \pm 0.024$ & $\mathbf{1 3 . 2 5 1} \pm 0.531$ \\
$15: 48$ & $\mathbf{0 . 9 3 7} \pm 0.048$ & $\mathbf{1 2 . 7 4 1} \pm 0.750$ & $15: 58$ & $\mathbf{1 . 0 8 0} \pm 0.034$ & $\mathbf{1 7 . 8 4 2} \pm 0.892$ \\
$16: 50$ & $\mathbf{0 . 8 8 0} \pm 0.044$ & $\mathbf{1 1 . 9 5 1} \pm 0.677$ & $17: 03$ & $\mathbf{0 . 7 7 0} \pm 0.036$ & $\mathbf{1 2 . 5 8 4} \pm 0.605$ \\
$17: 55$ & $\mathbf{0 . 7 3 4} \pm 0.045$ & $\mathbf{9 . 8 9 8} \pm 0.616$ & $18: 03$ & $\mathbf{0 . 7 6 7} \pm 0.029$ & $\mathbf{1 2 . 5 5 8} \pm 0.517$ \\
\hline
\end{tabular}

\begin{tabular}{|c|c|c|c|c|c|}
\hline \multicolumn{3}{|c|}{ Calligonum CFN 20.06.2000 } & \multicolumn{3}{|c|}{ Calligonum CFU 20.06.2000 } \\
\hline Zeit & $\begin{array}{l}\text { Transpiration } \\
{\left[\mathrm{mmol}^{*} \mathrm{~m}^{-2 *} \mathrm{~s}^{-1}\right]}\end{array}$ & $\begin{array}{l}\text { Transpiration } \\
{\left[\mu \mathrm{mol}^{*} \mathrm{~g}^{-2 *} \mathrm{~s}^{-1}\right]}\end{array}$ & Zeit & $\begin{array}{l}\text { Transpiration } \\
{\left[\mathrm{mmol}^{*} \mathrm{~m}^{-2 *} \mathrm{~s}^{-1}\right]}\end{array}$ & $\begin{array}{l}\text { Transpiration } \\
{\left[\mu \mathrm{mol}^{*} \mathrm{~g}^{-2 *} \mathrm{~s}^{-1}\right]}\end{array}$ \\
\hline 9:50 & $0.106 \pm 0.007$ & $1.250 \pm 0.086$ & 10:20 & $0.083 \pm 0.004$ & $1.049 \pm 0.055$ \\
\hline $10: 55$ & $0.221 \pm 0.012$ & $2.612 \pm 0.156$ & 11:31 & $0.300 \pm 0.014$ & $3.761 \pm 0.173$ \\
\hline $12: 00$ & $0.291 \pm 0.014$ & $3.422 \pm 0.176$ & $12: 33$ & $0.321 \pm 0.015$ & $4.029 \pm 0.174$ \\
\hline 13:01 & $0.366 \pm 0.017$ & $4.311 \pm 0.219$ & 13:32 & $0.250 \pm 0.010$ & $3.169 \pm 0.167$ \\
\hline 14:15 & $0.415 \pm 0.019$ & $4.895 \pm 0.248$ & $15: 29$ & $0.478 \pm 0.025$ & $6.068 \pm 0.400$ \\
\hline $15: 30$ & $0.416 \pm 0.018$ & $4.907 \pm 0.238$ & $16: 42$ & $0.413 \pm 0.020$ & $\mathbf{5 . 2 5 9} \pm 0.356$ \\
\hline $16: 35$ & $0.398 \pm 0.021$ & $4.699 \pm 0.274$ & $17: 38$ & $0.365 \pm 0.016$ & $4.631 \pm 0.261$ \\
\hline $17: 42$ & $0.362 \pm 0.019$ & $4.260 \pm 0.230$ & 18:48 & $0.273 \pm 0.009$ & $3.444 \pm 0.148$ \\
\hline 18:52 & $0.317 \pm 0.016$ & $3.726 \pm 0.200$ & & & \\
\hline
\end{tabular}

\begin{tabular}{|c|c|c|c|c|c|}
\hline \multicolumn{3}{|c|}{ Calligonum CFN $03 .($} & \multicolumn{3}{|c|}{ Calligonum CFU 03.08.2000 } \\
\hline Zeit & $\begin{array}{l}\text { Transpiration } \\
{\left[\mathrm{mmol}^{*} \mathrm{~m}^{-2 *} \mathrm{~s}^{-1}\right]}\end{array}$ & $\begin{array}{l}\text { Transpiration } \\
{\left[\mu \mathrm{mol}^{*} \mathrm{~g}^{-2 *} \mathrm{~s}^{-1}\right]}\end{array}$ & Zeit & $\begin{array}{l}\text { Transpiration } \\
{\left[\mathrm{mmol}^{*} \mathrm{~m}^{-2 *} \mathrm{~s}^{-1}\right]}\end{array}$ & $\begin{array}{l}\text { Transpiration } \\
{\left[\mu \mathrm{mol}^{*} \mathrm{~g}^{-2 *} \mathrm{~s}^{-1}\right]}\end{array}$ \\
\hline 10:10 & $0.296 \pm 0.023$ & $2.103 \pm 0.240$ & $10: 40$ & $0.178 \pm 0.025$ & $1.214 \pm 0.168$ \\
\hline 11:11 & $0.448 \pm 0.044$ & $3.185 \pm 0.430$ & 11:54 & $0.448 \pm 0.024$ & $3.095 \pm 0.197$ \\
\hline 12:07 & $\mathbf{0 . 6 2 2} \pm 0.056$ & $4.465 \pm 0.589$ & 13:17 & $0.584 \pm 0.025$ & $4.005 \pm 0.216$ \\
\hline 13:10 & $0.686 \pm 0.067$ & $4.970 \pm 0.737$ & $15: 25$ & $0.671 \pm 0.050$ & $4.551 \pm 0.328$ \\
\hline 15:03 & $0.778 \pm 0.091$ & $\mathbf{5 . 7 2 1} \pm 1.030$ & 16:37 & $0.474 \pm 0.035$ & $3.248 \pm 0.267$ \\
\hline 16:14 & $0.803 \pm 0.088$ & $\mathbf{5 . 8 8 9} \pm 1.025$ & $17: 43$ & $0.416 \pm 0.019$ & $2.861 \pm 0.180$ \\
\hline $17: 21$ & $0.768 \pm 0.078$ & $5.625 \pm 0.909$ & $18: 40$ & $0.363 \pm 0.027$ & $2.495 \pm 0.204$ \\
\hline 18:21 & $\mathbf{0 . 6 6 9} \pm 0.080$ & $4.923 \pm 0.911$ & & & \\
\hline 19:21 & $0.523 \pm 0.073$ & $3.906 \pm 0.777$ & & & \\
\hline
\end{tabular}

\begin{tabular}{ccc|ccc}
\hline \multicolumn{2}{c|}{ Calligonum CFN } & $\mathbf{1 2 . 0 9 . 2 0 0 0}$ & \multicolumn{3}{c}{ Calligonum CFU } \\
\hline Zeit & $\begin{array}{c}\text { Transpiration } \\
{\left[\mathrm{mmol}^{*} \mathrm{~m}^{-2 *} \mathrm{~s}^{-1}\right]}\end{array}$ & $\begin{array}{c}\text { Transpiration } \\
{\left[\mu \mathrm{mol}^{*} \mathrm{~g}^{-2 *} \mathrm{~s}^{-1}\right]}\end{array}$ & Zeit & $\begin{array}{l}\text { Transpiration } \\
{\left[\mathrm{mmol}^{*} \mathrm{~m}^{-2 *} \mathrm{~s}^{-1}\right]}\end{array}$ & $\begin{array}{l}\text { Transpiration } \\
{\left[\mu \mathrm{mol}^{*} \mathrm{~g}^{-2 *} \mathrm{~s}^{-1}\right]}\end{array}$ \\
\hline $10: 46$ & $\mathbf{0 . 3 1 9} \pm 0.022$ & $\mathbf{2 . 2 3 8} \pm 0.181$ & $10: 48$ & $\mathbf{0 . 4 2 5} \pm 0.034$ & $\mathbf{2 . 8 3 6} \pm 0.227$ \\
$11: 50$ & $\mathbf{0 . 5 0 0} \pm 0.026$ & $\mathbf{3 . 5 5 0} \pm 0.278$ & $11: 51$ & $\mathbf{0 . 6 1 6} \pm 0.031$ & $\mathbf{4 . 1 4 5} \pm 0.245$ \\
$13: 03$ & $\mathbf{0 . 6 1 8} \pm 0.043$ & $\mathbf{4 . 4 2 5} \pm 0.461$ & $13: 00$ & $\mathbf{0 . 7 6 0} \pm 0.051$ & $\mathbf{5 . 1 1 3} \pm 0.381$ \\
$14: 58$ & $\mathbf{0 . 7 7 9} \pm 0.052$ & $\mathbf{5 . 6 4 7} \pm 0.631$ & $14: 53$ & $\mathbf{0 . 8 2 4} \pm 0.037$ & $\mathbf{5 . 5 2 6} \pm 0.265$ \\
$16: 02$ & $\mathbf{0 . 7 5 7} \pm 0.057$ & $\mathbf{5 . 5 3 7} \pm 0.678$ & $15: 57$ & $\mathbf{0 . 8 3 6} \pm 0.044$ & $\mathbf{5 . 6 2 4} \pm 0.313$ \\
$17: 08$ & $\mathbf{0 . 7 3 9} \pm 0.046$ & $\mathbf{5 . 3 8 1} \pm 0.593$ & $16: 58$ & $\mathbf{0 . 7 6 4} \pm 0.043$ & $\mathbf{5 . 1 1 4} \pm 0.285$ \\
$18: 11$ & $\mathbf{0 . 6 8 5} \pm 0.040$ & $\mathbf{4 . 9 6 9} \pm 0.518$ & $18: 14$ & $\mathbf{0 . 5 9 8} \pm 0.035$ & $\mathbf{3 . 9 9 9} \pm 0.217$ \\
$19: 15$ & $\mathbf{0 . 5 9 7} \pm 0.033$ & $\mathbf{4 . 3 3 6} \pm 0.439$ & $18: 49$ & $\mathbf{0 . 5 2 6} \pm 0.027$ & $\mathbf{3 . 5 2 5} \pm 0.175$ \\
\hline
\end{tabular}


Tab. 24: Stomatäre Leitfähigkeit von Calligonum caput-medusae 2000.

Mittelwerte aus 18 Messungen (6 Pflanzen mit je 3 Parallelen) mit Standardfehler (se). Die Flächenkürzel bedeuten: $\mathbf{C}$ Calligonum caput-medusae, $\mathbf{F}$ bewässert (flooded), $\mathbf{N}$ nicht genutzt (not used), U genutzt (used).

\begin{tabular}{|c|c|c|c|c|c|}
\hline \multicolumn{3}{|c|}{ Calligonum CFN 17.05.2000 } & \multicolumn{3}{|c|}{ Calligonum CFU 17.05.2000 } \\
\hline Zeit & $\begin{array}{l}\text { Leitfähigkeit } \\
{\left[\mathrm{mmol}^{*} \mathrm{~m}^{-2 *} \mathrm{~s}^{-1}\right]}\end{array}$ & $\begin{array}{l}\text { Leitfähigkeit } \\
{\left[\mathrm{mmol}^{*} \mathrm{~g}^{-2 *} \mathrm{~s}^{-1}\right]}\end{array}$ & Zeit & $\begin{array}{l}\text { Leitfähigkeit } \\
{\left[\mathrm{mmol}^{*} \mathrm{~m}^{-2 *} \mathrm{~s}^{-1}\right]}\end{array}$ & $\begin{array}{l}\text { Leitfähigkeit } \\
{\left[\mathrm{mmol}^{*} \mathrm{~g}^{-2 *} \mathrm{~s}^{-1}\right]}\end{array}$ \\
\hline 10:01 & $21.172 \pm 1.166$ & $\mathbf{0 . 2 8 7} \pm 0.017$ & 10:17 & $24.605 \pm 1.255$ & $0.402 \pm 0.020$ \\
\hline $11: 00$ & $19.374 \pm 1.143$ & 0.262 & 11:16 & 0.867 & $0.389 \pm 0.019$ \\
\hline 12:01 & $19.679 \pm 1.158$ & $0.266 \pm 0.017$ & $12: 23$ & $23.076 \pm 0.917$ & $0.378 \pm 0.015$ \\
\hline $12: 57$ & $21.081 \pm 0.947$ & $0.286 \pm 0.015$ & 13:21 & $20.994 \pm 0.843$ & $0.344 \pm 0.015$ \\
\hline $14: 14$ & $21.424 \pm 0.847$ & $0.293 \pm 0.017$ & $14: 54$ & $17.674 \pm 0.626$ & $0.289 \pm 0.011$ \\
\hline $15: 48$ & $19.709 \pm 0.924$ & $0.268 \pm 0.016$ & $15: 58$ & $20.872 \pm 0.656$ & $\mathbf{0 . 3 4 3} \pm 0.015$ \\
\hline $16: 50$ & $18.568 \pm 1.017$ & $0.253 \pm 0.016$ & $17: 03$ & $15.975 \pm 0.614$ & $0.261 \pm 0.011$ \\
\hline 17:55 & $15.520 \pm 0.886$ & $0.210 \pm 0.012$ & 18:03 & $16.364 \pm 0.553$ & $0.268 \pm 0.010$ \\
\hline
\end{tabular}

\begin{tabular}{|c|c|c|c|c|c|}
\hline \multicolumn{3}{|c|}{ Calligonum CFN 20.06.2000 } & \multicolumn{3}{|c|}{ Calligonum CFU 20.06.2000 } \\
\hline Zeit & $\begin{array}{l}\text { Leitfähigkeit } \\
{\left[\mathrm{mmol}^{*} \mathrm{~m}^{-2 *} \mathrm{~s}^{-1}\right]}\end{array}$ & $\begin{array}{l}\text { Leitfähigkeit } \\
{\left[\mathrm{mmol}^{*} \mathrm{~g}^{-2 *} \mathrm{~s}^{-1}\right]}\end{array}$ & Zeit & $\begin{array}{l}\text { Leitfähigkeit } \\
{\left[\mathrm{mmol}^{*} \mathrm{~m}^{-2 *} \mathrm{~s}^{-1}\right]}\end{array}$ & $\begin{array}{l}\text { Leitfähigkeit } \\
{\left[\mathrm{mmol}^{*} \mathrm{~g}^{-2 *} \mathrm{~s}^{-1}\right]}\end{array}$ \\
\hline $9: 50$ & $\mathbf{5 . 0 2 8} \pm 0.296$ & $0.059 \pm 0.004$ & 10:20 & $3.403 \pm 0.148$ & $0.043 \pm 0.003$ \\
\hline $10: 55$ & $6.355 \pm 0.272$ & $0.075 \pm 0.003$ & $11: 31$ & $7.980 \pm 0.359$ & $0.100 \pm 0.004$ \\
\hline $12: 00$ & $6.598 \pm 0.339$ & $0.077 \pm 0.004$ & $12: 33$ & $6.798 \pm 0.295$ & $0.085 \pm 0.004$ \\
\hline 13:01 & $7.027 \pm 0.358$ & $0.083 \pm 0.004$ & 13:32 & $4.284 \pm 0.164$ & $0.054 \pm 0.003$ \\
\hline $14: 15$ & $7.298 \pm 0.374$ & $0.086 \pm 0.005$ & $15: 29$ & $8.053 \pm 0.475$ & $0.102 \pm 0.007$ \\
\hline $15: 30$ & $6.798 \pm 0.351$ & $0.080 \pm 0.005$ & $16: 42$ & $6.181 \pm 0.308$ & $0.078 \pm 0.005$ \\
\hline $16: 35$ & $6.242 \pm 0.346$ & $0.074 \pm 0.005$ & $17: 38$ & $5.914 \pm 0.272$ & $0.075 \pm 0.004$ \\
\hline $17: 42$ & $5.898 \pm 0.306$ & $0.069 \pm 0.004$ & $18: 48$ & $5.141 \pm 0.191$ & $0.065 \pm 0.003$ \\
\hline $18: 52$ & $5.445 \pm 0.253$ & $0.064 \pm 0.003$ & & & \\
\hline
\end{tabular}

\begin{tabular}{|c|c|c|c|c|c|}
\hline \multicolumn{2}{|c|}{ Calligonum CFN } & 03.08 .2000 & \multicolumn{3}{|c|}{ Calligonum CFU 03.08.2000 } \\
\hline Zeit & $\begin{array}{l}\text { Leitfähigkeit } \\
{\left[\mathrm{mmol}^{*} \mathrm{~m}^{-2 *} \mathrm{~s}^{-1}\right]}\end{array}$ & $\begin{array}{l}\text { Leitfähigkeit } \\
{\left[\mathrm{mmol}^{*} \mathrm{~g}^{-2 *} \mathrm{~s}^{-1}\right]}\end{array}$ & Zeit & $\begin{array}{l}\text { Leitfähigkeit } \\
{\left[\mathrm{mmol}^{*} \mathrm{~m}^{-2 *} \mathrm{~s}^{-1}\right]}\end{array}$ & $\begin{array}{l}\text { Leitfähigkeit } \\
{\left[\mathrm{mmol}^{*} \mathrm{~g}^{-2 *} \mathrm{~s}^{-1}\right]}\end{array}$ \\
\hline $10: 10$ & $24.489 \pm 2.079$ & $0.176 \pm 0.022$ & $10: 40$ & $8.342 \pm 0.583$ & $0.057 \pm 0.004$ \\
\hline 11:11 & $17.907 \pm 1.499$ & $0.128 \pm 0.016$ & 11:54 & $9.527 \pm 0.766$ & $0.065 \pm 0.006$ \\
\hline $12: 07$ & $16.632 \pm 1.794$ & $0.121 \pm 0.019$ & 13:17 & $11.761 \pm 0.585$ & $0.080 \pm 0.005$ \\
\hline $13: 10$ & $16.370 \pm 1.915$ & $0.120 \pm 0.021$ & $15: 25$ & $12.100 \pm 1.052$ & $0.083 \pm 0.007$ \\
\hline $15: 03$ & $15.281 \pm 2.064$ & $0.113 \pm 0.023$ & $16: 37$ & $7.299 \pm 0.490$ & $0.050 \pm 0.004$ \\
\hline $16: 14$ & $14.632 \pm 1.527$ & $0.107 \pm 0.017$ & $17: 43$ & $7.977 \pm 0.485$ & $0.055 \pm 0.004$ \\
\hline $17: 21$ & $13.890 \pm 1.429$ & $0.101 \pm 0.016$ & $18: 40$ & $7.751 \pm 0.674$ & $0.053 \pm 0.005$ \\
\hline 18:21 & $14.773 \pm 2.228$ & $0.110 \pm 0.024$ & & & \\
\hline 19:21 & $12.338 \pm 1.919$ & $0.093 \pm 0.020$ & & & \\
\hline
\end{tabular}

\begin{tabular}{|c|c|c|c|c|c|}
\hline \multicolumn{3}{|c|}{ Calligonum CFN 12.09.2000 } & \multicolumn{3}{|c|}{ Calligonum CFU 12.09 .2000} \\
\hline Zeit & $\begin{array}{l}\text { Leitfähigkeit } \\
{\left[\mathrm{mmol}^{*} \mathrm{~m}^{-2 *} \mathrm{~s}^{-1}\right]}\end{array}$ & $\begin{array}{l}\text { Leitfähigkeit } \\
{\left[\mathrm{mmol}^{*} \mathrm{~g}^{-2 *} \mathrm{~s}^{-1}\right]}\end{array}$ & Zeit & $\begin{array}{l}\text { Leitfähigkeit } \\
{\left[\mathrm{mmol}^{*} \mathrm{~m}^{-2 *} \mathrm{~s}^{-1}\right]}\end{array}$ & $\begin{array}{l}\text { Leitfähigkeit } \\
{\left[\mathrm{mmol}^{*} \mathrm{~g}^{-2 *} \mathrm{~s}^{-1}\right]}\end{array}$ \\
\hline 10:46 & $19.602 \pm 1.326$ & $0.139 \pm 0.012$ & $10: 48$ & $25.173 \pm 2.090$ & $0.168 \pm 0.014$ \\
\hline $11: 50$ & $20.206 \pm 1.354$ & $0.145 \pm 0.015$ & 11:51 & $23.463 \pm 1.451$ & $0.158 \pm 0.011$ \\
\hline 13:03 & $18.868 \pm 1.359$ & $0.135 \pm 0.015$ & 13:00 & $20.775 \pm 1.145$ & $0.139 \pm 0.008$ \\
\hline $14: 58$ & $18.932 \pm 1.066$ & $0.137 \pm 0.014$ & $14: 53$ & 17.141 & $0.115 \pm 0.007$ \\
\hline 16:02 & $17.685 \pm$ & 0.12 & $15: 57$ & 17.2 & $0.116 \pm 0.009$ \\
\hline 17:08 & $19.834 \pm 1.513$ & $0.143 \pm 0.017$ & $16: 58$ & $17.235 \pm$ & $0.115 \pm 0.008$ \\
\hline 18:11 & $17.319 \pm 1.158$ & $0.125 \pm 0.014$ & $18: 14$ & $16.371 \pm 1.074$ & $0.109 \pm 0.006$ \\
\hline 19:15 & $17.620 \pm 1.082$ & $0.127 \pm 0.013$ & $18: 49$ & $16.266 \pm 0.934$ & $0.109 \pm 0.006$ \\
\hline
\end{tabular}


Tab. 25: Transpiration von Tamarix ramosissima 2000.

Mittelwerte aus 18 Messungen (6 Pflanzen mit je 3 Parallelen) mit Standardfehler (se). Die Flächenkürzel bedeuten: $\mathbf{T}$ Tamarix ramosissima, $\mathbf{F}$ bewässert (flooded), $\mathbf{N}$ nicht genutzt (not used), U genutzt (used).

\begin{tabular}{ccc|ccc}
\hline \multicolumn{3}{c|}{ Tamarix TFN 18.05.2000 } & \multicolumn{3}{c}{ Tamarix TFU 18.05.2000 } \\
\hline Zeit & $\begin{array}{c}\text { Transpiration } \\
{\left[\mathrm{mmol}^{*} \mathrm{~m}^{-2 *} \mathrm{~s}^{-1}\right]}\end{array}$ & $\begin{array}{c}\text { Transpiration } \\
{\left[\mu \mathrm{mol}^{*} \mathrm{~g}^{-2 *} \mathrm{~s}^{-1}\right]}\end{array}$ & Zeit & $\begin{array}{c}\text { Transpiration } \\
{\left[\mathrm{mmol}^{*} \mathrm{~m}^{-2 *} \mathrm{~s}^{-1}\right]}\end{array}$ & $\begin{array}{l}\text { Transpiration } \\
{\left[\mu \mathrm{mol}^{*} \mathrm{~g}^{-2 *} \mathrm{~s}^{-1}\right]}\end{array}$ \\
\hline $10: 25$ & $\mathbf{0 . 5 8 9} \pm 0.038$ & $\mathbf{3 . 9 7 6} \pm 0.260$ & $10: 57$ & $\mathbf{1 . 1 8 9} \pm 0.084$ & $\mathbf{6 . 7 8 3} \pm 0.508$ \\
$11: 38$ & $\mathbf{0 . 8 2 7} \pm 0.039$ & $\mathbf{5 . 6 0 2} \pm 0.299$ & $11: 58$ & $\mathbf{1 . 3 8 4} \pm 0.091$ & $\mathbf{7 . 8 5 1} \pm 0.537$ \\
$12: 40$ & $\mathbf{1 . 1 2 5} \pm 0.067$ & $\mathbf{7 . 6 0 7} \pm 0.467$ & $12: 45$ & $\mathbf{1 . 7 6 2} \pm 0.086$ & $\mathbf{9 . 9 4 9} \pm 0.486$ \\
$14: 20$ & $\mathbf{1 . 3 2 6} \pm 0.073$ & $\mathbf{8 . 9 4 4} \pm 0.482$ & $14: 21$ & $\mathbf{1 . 6 5 0} \pm 0.089$ & $\mathbf{9 . 3 8 8} \pm 0.565$ \\
$15: 17$ & $\mathbf{1 . 4 6 7} \pm 0.070$ & $\mathbf{9 . 8 7 7} \pm 0.456$ & $15: 50$ & $\mathbf{1 . 3 0 0} \pm 0.099$ & $\mathbf{7 . 2 8 6} \pm 0.519$ \\
$16: 25$ & $\mathbf{1 . 3 6 9} \pm 0.068$ & $\mathbf{9 . 2 2 3} \pm 0.446$ & $16: 31$ & $\mathbf{1 . 6 4 7} \pm 0.060$ & $\mathbf{9 . 3 1 4} \pm 0.343$ \\
$17: 26$ & $\mathbf{1 . 2 0 9} \pm 0.056$ & $\mathbf{8 . 1 4 2} \pm 0.364$ & $17: 34$ & $\mathbf{1 . 6 9 7} \pm 0.077$ & $\mathbf{9 . 5 8 9} \pm 0.436$ \\
\hline
\end{tabular}

\begin{tabular}{ccc|ccr}
\hline \multicolumn{3}{c|}{ Tamarix TFN 19.09.2000 } & \multicolumn{3}{c}{ Tamarix TFU 19.09.2000 } \\
\hline Zeit & $\begin{array}{c}\text { Transpiration } \\
{\left[\mathrm{mmol}^{*} \mathrm{~m}^{-2 *} \mathrm{~s}^{-1}\right]}\end{array}$ & $\begin{array}{c}\text { Transpiration } \\
{\left[\mu \mathrm{mol}^{*} \mathrm{~g}^{-2 *} \mathrm{~s}^{-1}\right]}\end{array}$ & Zeit & $\begin{array}{c}\text { Transpiration } \\
{\left[\mathrm{mmol}^{*} \mathrm{~m}^{-2 *} \mathrm{~s}^{-1}\right]}\end{array}$ & $\begin{array}{l}\text { Transpiration } \\
{\left[\mu \mathrm{mol}^{*} \mathrm{~g}^{-2 *} \mathrm{~s}^{-1}\right]}\end{array}$ \\
\hline $10: 38$ & $\mathbf{0 . 4 4 5} \pm 0.036$ & $\mathbf{2 . 5 7 9} \pm 0.270$ & $11: 32$ & $\mathbf{0 . 9 8 8} \pm 0.103$ & $\mathbf{5 . 5 5 3} \pm 0.657$ \\
$11: 53$ & $\mathbf{0 . 8 1 5} \pm 0.073$ & $\mathbf{4 . 7 3 4} \pm 0.532$ & $12: 47$ & $\mathbf{1 . 7 5 7} \pm 0.141$ & $\mathbf{9 . 5 1 8} \pm 0.698$ \\
$13: 08$ & $\mathbf{0 . 9 8 3} \pm 0.094$ & $\mathbf{5 . 7 0 8} \pm 0.669$ & $14: 33$ & $\mathbf{2 . 4 3 7} \pm 0.253$ & $\mathbf{1 2 . 8 9 6} \pm 1.044$ \\
$15: 10$ & $\mathbf{1 . 1 2 4} \pm 0.107$ & $\mathbf{6 . 5 2 5} \pm 0.756$ & $15: 59$ & $\mathbf{2 . 2 7 6} \pm 0.187$ & $\mathbf{1 2 . 1 9 5} \pm 0.795$ \\
$16: 19$ & $\mathbf{1 . 0 7 5} \pm 0.096$ & $\mathbf{6 . 2 4 5} \pm 0.689$ & $17: 01$ & $\mathbf{2 . 1 5 8} \pm 0.183$ & $\mathbf{1 1 . 5 3 8} \pm 0.800$ \\
$17: 20$ & $\mathbf{1 . 0 3 3} \pm 0.093$ & $\mathbf{5 . 9 9 1} \pm 0.669$ & $18: 01$ & $\mathbf{1 . 7 9 7} \pm 0.151$ & $\mathbf{9 . 7 6 2} \pm 0.830$ \\
$18: 21$ & $\mathbf{0 . 8 9 5} \pm 0.078$ & $\mathbf{5 . 1 8 8} \pm 0.561$ & $18: 58$ & $\mathbf{1 . 7 3 3} \pm 0.144$ & $\mathbf{9 . 3 9 2} \pm 0.758$ \\
$19: 20$ & $\mathbf{0 . 7 1 8} \pm 0.060$ & $\mathbf{4 . 1 4 9} \pm 0.429$ & $19: 25$ & $\mathbf{1 . 2 6 2} \pm 0.098$ & $\mathbf{6 . 8 7 5} \pm 0.520$ \\
\hline
\end{tabular}

Tab. 26: Stomatäre Leitfähigkeit von Tamarix ramosissima 2000.

Mittelwerte aus 18 Messungen (6 Pflanzen mit je 3 Parallelen) mit Standardfehler (se). Die Flächenkürzel bedeuten: $\mathbf{T}$ Tamarix ramosissima, $\mathbf{F}$ bewässert (flooded), $\mathbf{N}$ nicht genutzt (not used), U genutzt (used).

\begin{tabular}{ccc|ccc}
\hline \multicolumn{3}{c|}{ Tamarix TFN } & $\mathbf{1 8 . 0 5 . 2 0 0 0}$ & \multicolumn{3}{c}{ Tamarix TFU } & $\mathbf{1 8 . 0 5 . 2 0 0 0}$ \\
\hline Zeit & $\begin{array}{c}\text { Leitfähigkeit } \\
{\left[\mathrm{mmol}^{*} \mathrm{~m}^{-2 *} \mathrm{~s}^{-1}\right]}\end{array}$ & $\begin{array}{c}\text { Leitfähigkeit } \\
{\left[\mathrm{mmol}^{*} \mathrm{~g}^{-2} \mathrm{~s}^{-1}\right]}\end{array}$ & Zeit & $\begin{array}{c}\text { Leitfähigkeit } \\
{\left[\mathrm{mmol}^{*} \mathrm{~m}^{-2 *} \mathrm{~s}^{-1}\right]}\end{array}$ & $\begin{array}{l}\text { Leitfähigkeit } \\
{\left[\mathrm{mmol}^{*} \mathrm{~g}^{-2 *} \mathrm{~s}^{-1}\right]}\end{array}$ \\
\hline $10: 25$ & $\mathbf{2 7 . 0 0 3} \pm 1.870$ & $\mathbf{0 . 1 8 3} \pm 0.013$ & $10: 57$ & $\mathbf{4 7 . 8 7 5} \pm 2.511$ & $\mathbf{0 . 2 7 3} \pm 0.017$ \\
$11: 38$ & $\mathbf{2 4 . 5 7 3} \pm 1.017$ & $\mathbf{0 . 1 6 8} \pm 0.010$ & $11: 58$ & $35.863 \pm 1.930$ & $\mathbf{0 . 2 0 4} \pm 0.012$ \\
$12: 40$ & $\mathbf{2 5 . 6 6 4} \pm 1.509$ & $\mathbf{0 . 1 7 4} \pm 0.011$ & $12: 45$ & $\mathbf{4 0 . 3 7 6} \pm 2.270$ & $\mathbf{0 . 2 3 0} \pm 0.014$ \\
$14: 20$ & $\mathbf{2 6 . 2 1 3} \pm 1.564$ & $\mathbf{0 . 1 7 7} \pm 0.010$ & $14: 21$ & $\mathbf{3 7 . 8 1 0} \pm 2.334$ & $\mathbf{0 . 2 1 6} \pm 0.015$ \\
$15: 17$ & $\mathbf{2 6 . 4 8 9} \pm 1.380$ & $\mathbf{0 . 1 7 9} \pm 0.010$ & $15: 50$ & $\mathbf{2 8 . 6 0 1} \pm 1.994$ & $\mathbf{0 . 1 6 1} \pm 0.011$ \\
$16: 25$ & $\mathbf{2 5 . 9 1 7} \pm 1.465$ & $\mathbf{0 . 1 7 5} \pm 0.010$ & $16: 31$ & $\mathbf{3 2 . 9 0 8} \pm 1.068$ & $\mathbf{0 . 1 8 6} \pm 0.007$ \\
$17: 26$ & $\mathbf{2 2 . 9 8 5} \pm 1.246$ & $\mathbf{0 . 1 5 5} \pm 0.008$ & $17: 34$ & $\mathbf{3 1 . 9 6 1 \pm 1 . 4 8 7}$ & $\mathbf{0 . 1 8 2} \pm 0.009$ \\
\hline
\end{tabular}

\begin{tabular}{ccc|ccc}
\hline \multicolumn{3}{c|}{ Tamarix TFN 19.09.2000 } & \multicolumn{3}{c}{ Tamarix TFU 19.09.2000 } \\
\hline Zeit & $\begin{array}{c}\text { Leitfähigkeit } \\
{\left[\mathrm{mmol}^{*} \mathrm{~m}^{-2 *} \mathrm{~s}^{-1}\right]}\end{array}$ & $\begin{array}{c}\text { Leitfähigkeit } \\
{\left[\mathrm{mmol}^{*} \mathrm{~g}^{-2 *} \mathrm{~s}^{-1}\right]}\end{array}$ & Zeit & $\begin{array}{c}\text { Leitfähigkeit } \\
{\left[\mathrm{mmol}^{*} \mathrm{~m}^{-2 *} \mathrm{~s}^{-1}\right]}\end{array}$ & $\begin{array}{l}\text { Leitfähigkeit } \\
{\left[\mathrm{mmol}^{*} \mathrm{~g}^{-2 *} \mathrm{~s}^{-1}\right]}\end{array}$ \\
\hline $10: 38$ & $\mathbf{2 6 . 7 9 0} \pm 2.318$ & $\mathbf{0 . 1 5 5} \pm 0.017$ & $11: 32$ & $\mathbf{5 3 . 0 0 3} \pm 5.542$ & $\mathbf{0 . 2 9 3} \pm 0.033$ \\
$11: 53$ & $\mathbf{2 9 . 1 1 5} \pm 3.042$ & $\mathbf{0 . 1 7 0} \pm 0.021$ & $12: 47$ & $\mathbf{5 2 . 2 2 2} \pm 5.073$ & $\mathbf{0 . 2 8 3} \pm 0.028$ \\
$13: 08$ & $\mathbf{2 5 . 1 2 0} \pm 2.609$ & $\mathbf{0 . 1 4 7} \pm 0.018$ & $14: 33$ & $\mathbf{4 6 . 5 8 9} \pm 4.444$ & $\mathbf{0 . 2 4 9} \pm 0.021$ \\
$15: 10$ & $\mathbf{2 3 . 5 3 5} \pm 2.695$ & $\mathbf{0 . 1 3 7} \pm 0.019$ & $15: 59$ & $\mathbf{3 8 . 2 9 1} \pm 2.848$ & $\mathbf{0 . 2 0 7} \pm 0.014$ \\
$16: 19$ & $\mathbf{2 2 . 6 0 0} \pm 2.331$ & $\mathbf{0 . 1 3 2} \pm 0.017$ & $17: 01$ & $\mathbf{3 9 . 0 2 1} \pm 2.926$ & $\mathbf{0 . 2 1 1} \pm 0.013$ \\
$17: 20$ & $\mathbf{2 3 . 4 4 4} \pm 2.587$ & $\mathbf{0 . 1 3 7} \pm 0.018$ & $18: 01$ & $\mathbf{3 7 . 1 6 6} \pm 2.662$ & $\mathbf{0 . 2 0 2} \pm 0.013$ \\
$18: 21$ & $\mathbf{2 3 . 7 2 7} \pm 2.395$ & $\mathbf{0 . 1 3 8} \pm 0.017$ & $18: 58$ & $\mathbf{4 0 . 1 9 4} \pm 3.004$ & $\mathbf{0 . 2 1 9} \pm 0.016$ \\
$19: 20$ & $\mathbf{2 0 . 6 2 1} \pm 1.935$ & $\mathbf{0 . 1 2 0} \pm 0.014$ & $19: 25$ & $\mathbf{3 3 . 6 1 4} \pm 2.685$ & $\mathbf{0 . 1 8 4} \pm 0.016$ \\
\hline
\end{tabular}


Tab. 27: Transpiration von Populus euphratica 2000.

Mittelwerte aus 18 Messungen (6 Pflanzen mit je 3 Parallelen) mit Standardfehler (se). Die Flächenkürzel bedeuten: T Populus euphratica, F bewässert (flooded), N nicht genutzt (not used), U genutzt (used).

\begin{tabular}{|c|c|c|c|c|c|}
\hline \multicolumn{3}{|c|}{ Populus PFN 29.05.2000 } & \multicolumn{3}{|c|}{ Populus PFU 29.05.2000 } \\
\hline Zeit & $\begin{array}{l}\text { Transpiration } \\
{\left[\mathrm{mmol}^{*} \mathrm{~m}^{-2 *} \mathrm{~s}^{-1}\right]}\end{array}$ & $\begin{array}{l}\text { Transpiration } \\
{\left[\mu \mathrm{mol}^{*} \mathrm{~g}^{-2 *} \mathrm{~s}^{-1}\right]}\end{array}$ & Zeit & $\begin{array}{l}\text { Transpiration } \\
{\left[\mathrm{mmol}^{*} \mathrm{~m}^{-2 *} \mathrm{~s}^{-1}\right]}\end{array}$ & $\begin{array}{l}\text { Transpiration } \\
{\left[\mu \mathrm{mol}^{*} \mathrm{~g}^{-2 *} \mathrm{~s}^{-1}\right]}\end{array}$ \\
\hline $10: 38$ & $1.231 \pm 0.095$ & $10.597 \pm 1.088$ & & & \\
\hline $11: 45$ & $1.888 \pm 0.116$ & $15.981 \pm 1.353$ & & & \\
\hline $12: 52$ & $2.518 \pm 0.130$ & $21.151 \pm 1.493$ & & & \\
\hline $14: 18$ & $2.944 \pm 0.153$ & $24.675 \pm 1.664$ & & & \\
\hline $15: 26$ & $3.010 \pm 0.156$ & $25.082 \pm 1.537$ & & & \\
\hline $16: 34$ & $2.599 \pm 0.141$ & $21.498 \pm 1.252$ & & & \\
\hline $17: 39$ & $2.613 \pm 0.120$ & $21.700 \pm 1.205$ & & & \\
\hline $18: 36$ & $2.365 \pm 0.134$ & $19.474 \pm 1.026$ & & & \\
\hline
\end{tabular}

\begin{tabular}{ccc|crr}
\hline \multicolumn{3}{c|}{ Populus PFN 06.07.2000 } & \multicolumn{3}{c}{ Populus PFU 06.07.2000 } \\
\hline Zeit & $\begin{array}{c}\text { Transpiration } \\
{\left[\mathrm{mmol}^{*} \mathrm{~m}^{-2 *} \mathrm{~s}^{-1}\right]}\end{array}$ & $\begin{array}{c}\text { Transpiration } \\
{\left[\mu \mathrm{mol}^{*} \mathrm{~g}^{-2 *} \mathrm{~s}^{-1}\right]}\end{array}$ & $\begin{array}{l}\text { Zeit } \\
\text { Transpiration } \\
{\left[\mathrm{mmol}^{*} \mathrm{~m}^{-2 *} \mathrm{~s}^{-1}\right]}\end{array}$ & $\begin{array}{l}\text { Transpiration } \\
{\left[\mu \mathrm{mol}^{*} \mathrm{~g}^{-2 *} \mathrm{~s}^{-1}\right]}\end{array}$ \\
\hline $10: 33$ & $\mathbf{1 . 0 2 8} \pm 0.051$ & $\mathbf{7 . 3 0 0} \pm 0.512$ & $10: 44$ & $\mathbf{1 . 0 7 6} \pm 0.085$ & $\mathbf{7 . 6 5 9} \pm 0.479$ \\
$11: 46$ & $\mathbf{1 . 4 0 6} \pm 0.063$ & $\mathbf{9 . 9 3 0} \pm 0.616$ & $11: 55$ & $\mathbf{1 . 9 2 0} \pm 0.078$ & $\mathbf{1 3 . 8 0 6} \pm 0.391$ \\
$12: 50$ & $\mathbf{1 . 6 9 0} \pm 0.100$ & $11.938 \pm 0.890$ & $12: 54$ & $\mathbf{1 . 3 0 0} \pm 0.080$ & $\mathbf{9 . 3 9 7} \pm 0.558$ \\
$14: 57$ & $\mathbf{2 . 0 9 2} \pm 0.128$ & $\mathbf{1 4 . 7 6 7} \pm 1.072$ & $14: 51$ & $\mathbf{2 . 0 9 0} \pm 0.159$ & $\mathbf{1 4 . 8 6 1} \pm 0.960$ \\
$16: 03$ & $\mathbf{1 . 6 1 5} \pm 0.159$ & $\mathbf{1 1 . 4 4 8} \pm 1.232$ & $16: 02$ & $\mathbf{2 . 0 6 8} \pm 0.230$ & $\mathbf{1 4 . 6 5 8} \pm 1.531$ \\
$17: 04$ & $\mathbf{1 . 4 5 9} \pm 0.119$ & $\mathbf{1 0 . 1 2 8} \pm 0.810$ & $17: 04$ & $\mathbf{1 . 6 7 5} \pm 0.182$ & $\mathbf{1 1 . 7 0 7} \pm 1.096$ \\
$18: 07$ & $\mathbf{1 . 4 3 3} \pm 0.107$ & $\mathbf{1 0 . 0 1 8} \pm 0.795$ & $18: 06$ & $\mathbf{1 . 6 3 7} \pm 0.173$ & $\mathbf{1 1 . 5 9 5} \pm 1.100$ \\
$19: 09$ & $\mathbf{1 . 0 0 9} \pm 0.079$ & $\mathbf{7 . 0 6 2} \pm 0.569$ & $19: 03$ & $\mathbf{1 . 2 9 0} \pm 0.143$ & $\mathbf{9 . 0 8 2} \pm 0.089$ \\
\hline
\end{tabular}

\begin{tabular}{|c|c|c|c|c|c|}
\hline \multicolumn{3}{|c|}{ Populus PFN 12.08.2000 } & \multicolumn{3}{|c|}{ Populus PFU 12.08.2000 } \\
\hline Zeit & $\begin{array}{l}\text { Transpiration } \\
{\left[\mathrm{mmol}^{*} \mathrm{~m}^{-2 *} \mathrm{~s}^{-1}\right]}\end{array}$ & $\begin{array}{l}\text { Transpiration } \\
{\left[\mu \mathrm{mol}{ }^{*} \mathrm{~g}^{-2 *} \mathrm{~s}^{-1}\right]}\end{array}$ & Zeit & $\begin{array}{l}\text { Transpiration } \\
{\left[\mathrm{mmol}^{*} \mathrm{~m}^{-2 \star} \mathrm{s}^{-1}\right]}\end{array}$ & $\begin{array}{l}\text { Transpiration } \\
{\left[\mu \mathrm{mol}^{*} \mathrm{~g}^{-2 *} \mathrm{~s}^{-1}\right]}\end{array}$ \\
\hline $11: 21$ & $1.826 \pm 0.061$ & $12.098 \pm 0.712$ & $11: 22$ & $3.357 \pm 0.098$ & $21.872 \pm 0.700$ \\
\hline $12: 21$ & $1.995 \pm 0.084$ & $13.246 \pm 0.8$ & $12: 32$ & $4.167 \pm 0.114$ & 27.0 \\
\hline $13: 16$ & $2.322 \pm 0.098$ & $15.275 \pm 0.879$ & $13: 43$ & $5 \pm 0.124$ & $7 \pm 0.834$ \\
\hline $15: 20$ & 44 & 19.1 & & 0.206 & $7 \pm 1.253$ \\
\hline $16: 24$ & .097 & $17.708 \pm 0.915$ & $16: 48$ & 0.205 & $9 \pm 1.149$ \\
\hline $17: 23$ & $2.591 \pm$ & $17.077 \pm 0.931$ & $18: 01$ & $3 \pm 0.151$ & $23.761 \pm 0.853$ \\
\hline $18: 20$ & $2.097 \pm 0.087$ & $13.739 \pm 0.747$ & $18: 55$ & $2.979 \pm 0.172$ & $19.198 \pm 0.896$ \\
\hline $19: 19$ & $1.576 \pm 0.091$ & $10.398 \pm 0.783$ & & & \\
\hline
\end{tabular}

\begin{tabular}{|c|c|c|c|c|c|}
\hline \multicolumn{3}{|c|}{ Populus PFN 25.09.2000 } & \multicolumn{3}{|c|}{ Populus PFU 25.09.2000 } \\
\hline Zeit & $\begin{array}{l}\text { Transpiration } \\
{\left[\mathrm{mmol}^{*} \mathrm{~m}^{-2 *} \mathrm{~s}^{-1}\right]}\end{array}$ & $\begin{array}{l}\text { Transpiration } \\
{\left[\mu \mathrm{mol}^{*} \mathrm{~g}^{-2 *} \mathrm{~s}^{-1}\right]}\end{array}$ & Zeit & $\begin{array}{l}\text { Transpiration } \\
{\left[\mathrm{mmol}^{*} \mathrm{~m}^{-2 *} \mathrm{~s}^{-1}\right]}\end{array}$ & $\begin{array}{l}\text { Transpiration } \\
{\left[\mu \mathrm{mol}^{*} \mathrm{~g}^{-2 *} \mathrm{~s}^{-1}\right]}\end{array}$ \\
\hline & $1.166 \pm 0.061$ & & & & $8.952 \pm 0.636$ \\
\hline & 083 & 15.1 & & & \\
\hline & 094 & $18.742 \pm$ & & & 11 \\
\hline & 8 & 18.5 & & & \\
\hline & \pm 0.060 & $15.679 \pm 1.702$ & & 0.087 & $10.549 \pm 0.770$ \\
\hline 18:58 & $1.359 \pm 0.080$ & $13.000 \pm 1.803$ & 19:04 & $1.290 \pm 0.070$ & $8.329 \pm 0.692$ \\
\hline
\end{tabular}


Tab. 28: Stomatäre Leitfähigkeit von Populus euphratica 2000.

Mittelwerte aus 18 Messungen (6 Pflanzen mit je 3 Parallelen) mit Standardfehler (se). Die Flächenkürzel bedeuten: T Populus euphratica, $\mathbf{F}$ bewässert (flooded), $\mathbf{N}$ nicht genutzt (not used), U genutzt (used).

\begin{tabular}{|c|c|c|c|c|c|}
\hline \multicolumn{3}{|c|}{ Populus PFN 29.05.2000 } & \multicolumn{3}{|c|}{ Populus PFU 29.05.2000 } \\
\hline Zeit & $\begin{array}{l}\text { Leitfähigkeit } \\
{\left[\mathrm{mmol}^{*} \mathrm{~m}^{-2 *} \mathrm{~s}^{-1}\right]}\end{array}$ & $\begin{array}{l}\text { Leitfähigkeit } \\
{\left[\mathrm{mmol}^{*} \mathrm{~g}^{-2 *} \mathrm{~s}^{-1}\right]}\end{array}$ & Zeit & $\begin{array}{l}\text { Leitfähigkeit } \\
{\left[\mathrm{mmol}^{*} \mathrm{~m}^{-2 *} \mathrm{~s}^{-1}\right]}\end{array}$ & $\begin{array}{l}\text { Leitfähigkeit } \\
{\left[\mathrm{mmol}^{*} \mathrm{~g}^{-2 *} \mathrm{~s}^{-1}\right]}\end{array}$ \\
\hline $10: 38$ & $58.894 \pm 2.617$ & $0.497 \pm 0.034$ & & & \\
\hline 11:45 & $59.906 \pm 1.942$ & $0.498 \pm 0.023$ & & & \\
\hline $12: 52$ & $61.311 \pm 2.976$ & $0.512 \pm 0.032$ & & & \\
\hline $14: 18$ & $61.189 \pm 2.821$ & $0.509 \pm 0.029$ & & & \\
\hline $15: 26$ & $59.178 \pm 3.621$ & $\mathbf{0 . 4 8 8} \pm 0.029$ & & & \\
\hline $16: 34$ & $\mathbf{5 2 . 4 3 3} \pm 3.179$ & $0.434 \pm 0.028$ & & & \\
\hline $17: 39$ & $51.311 \pm 3.020$ & $0.425 \pm 0.027$ & & & \\
\hline 18:36 & $47.183 \pm 2.963$ & $0.388 \pm 0.022$ & & & \\
\hline
\end{tabular}

\begin{tabular}{|c|c|c|c|c|c|}
\hline \multicolumn{3}{|c|}{ Populus PFN 06.07.2000 } & \multicolumn{3}{|c|}{ Populus PFU 06.07.2000 } \\
\hline Zeit & $\begin{array}{l}\text { Leitfähigkeit } \\
{\left[\mathrm{mmol}^{*} \mathrm{~m}^{-2 *} \mathrm{~s}^{-1}\right]}\end{array}$ & $\begin{array}{l}\text { Leitfähigkeit } \\
{\left[\mathrm{mmol}^{*} \mathrm{~g}^{-2 *} \mathrm{~s}^{-1}\right]}\end{array}$ & Zeit & $\begin{array}{l}\text { Leitfähigkeit } \\
{\left[\mathrm{mmol}^{*} \mathrm{~m}^{-2 *} \mathrm{~s}^{-1}\right]}\end{array}$ & $\begin{array}{l}\text { Leitfähigkeit } \\
{\left[\mathrm{mmol}^{*} \mathrm{~g}^{-2 *} \mathrm{~s}^{-1}\right]}\end{array}$ \\
\hline 10:33 & $64.622 \pm 2.617$ & $\mathbf{0 . 4 5 6} \pm 0.025$ & 10:44 & $57.600 \pm 3.715$ & $0.411 \pm 0.019$ \\
\hline $11: 46$ & $53.922 \pm 3.047$ & $0.381 \pm 0.027$ & $11: 55$ & $61.150 \pm 2.924$ & $0.439 \pm 0.016$ \\
\hline $12: 50$ & $45.072 \pm 2.713$ & $0.317 \pm 0.023$ & $12: 54$ & $35.483 \pm 2.207$ & $0.255 \pm 0.014$ \\
\hline $14: 57$ & $42.900 \pm 2.580$ & $0.303 \pm 0.021$ & $14: 51$ & $45.167 \pm 3.558$ & $0.321 \pm 0.021$ \\
\hline 16:03 & $30.083 \pm 3.411$ & $0.214 \pm 0.026$ & 16:02 & $39.972 \pm 4.769$ & $0.283 \pm 0.032$ \\
\hline 17:04 & $28.233 \pm 2.202$ & $0.197 \pm 0.016$ & 17:04 & $36.329 \pm 4.607$ & $0.253 \pm 0.029$ \\
\hline 18:07 & $31.517 \pm 2.466$ & $0.220 \pm 0.018$ & 18:06 & $35.644 \pm 4.328$ & $0.251 \pm 0.028$ \\
\hline 19:09 & $23.328 \pm 1.900$ & $0.163 \pm 0.014$ & 19:03 & $28.839 \pm 3.462$ & $0.203 \pm 0.022$ \\
\hline
\end{tabular}

\begin{tabular}{|c|c|c|c|c|c|}
\hline \multicolumn{3}{|c|}{ Populus PFN 12.08.2000 } & \multicolumn{3}{|c|}{ Populus PFU 12.08 .2000} \\
\hline Zeit & $\begin{array}{l}\text { Leitfähigkeit } \\
{\left[\mathrm{mmol}^{*} \mathrm{~m}^{-2 *} \mathrm{~s}^{-1}\right]}\end{array}$ & $\begin{array}{l}\text { Leitfähigkeit } \\
{\left[\mathrm{mmol}^{*} \mathrm{~g}^{-2 *} \mathrm{~s}^{-1}\right]}\end{array}$ & Zeit & $\begin{array}{l}\text { Leitfähigkeit } \\
{\left[\mathrm{mmol}^{*} \mathrm{~m}^{-2 *} \mathrm{~s}^{-1}\right]}\end{array}$ & $\begin{array}{l}\text { Leitfähigkeit } \\
{\left[\mathrm{mmol}^{*} \mathrm{~g}^{-2 *} \mathrm{~s}^{-1}\right]}\end{array}$ \\
\hline 11:21 & $66.028 \pm 2.053$ & $0.432 \pm 0.019$ & 11:22 & $101.883 \pm 2.543$ & $0.668 \pm 0.026$ \\
\hline $12: 21$ & $58.261 \pm 1.807$ & $\mathbf{0 . 3 8 2} \pm 0.018$ & $12: 32$ & $94.861 \pm 2.722$ & $0.618 \pm 0.019$ \\
\hline $13: 16$ & $58.422 \pm 1.689$ & $\mathbf{0 . 3 8 3} \pm 0.017$ & $13: 43$ & $75.133 \pm 2.589$ & $0.488 \pm 0.015$ \\
\hline $15: 20$ & $\mathbf{5 8 . 8 2 8} \pm 1.640$ & $0.386 \pm 0.018$ & $15: 34$ & $76.983 \pm 2.843$ & $0.501 \pm 0.018$ \\
\hline $16: 24$ & $55.872 \pm 2.558$ & $0.367 \pm 0.022$ & $16: 48$ & $72.828 \pm 3.345$ & $0.471 \pm 0.019$ \\
\hline $17: 23$ & $53.633 \pm 2.228$ & $0.352 \pm 0.020$ & 18:01 & $69.700 \pm 3.037$ & $0.452 \pm 0.018$ \\
\hline $18: 20$ & $45.633 \pm 2.162$ & $0.298 \pm 0.016$ & 18:55 & $63.406 \pm 3.847$ & $0.409 \pm 0.021$ \\
\hline 19:19 & $36.300 \pm 2.372$ & $\mathbf{0 . 2 3 9} \pm 0.019$ & & & \\
\hline
\end{tabular}

\begin{tabular}{ccc|ccc}
\hline \multicolumn{3}{c|}{ Populus PFN 25.09.2000 } & \multicolumn{3}{c}{ Populus PFU 25.09.2000 } \\
\hline Zeit & $\begin{array}{c}\text { Leitfähigkeit } \\
{\left[\mathrm{mmol}^{*} \mathrm{~m}^{-2 *} \mathrm{~s}^{-1}\right]}\end{array}$ & $\begin{array}{c}\text { Leitfähigkeit } \\
{\left[\mathrm{mmol}^{*} \mathrm{~g}^{-2 *} \mathrm{~s}^{-1}\right]}\end{array}$ & $\begin{array}{l}\text { Zeit } \\
\text { Leitfähigkeit } \\
{\left[\mathrm{mmol}^{*} \mathrm{~m}^{-2 *} \mathrm{~s}^{-1}\right]}\end{array}$ & $\begin{array}{l}\text { Leitfähigkeit } \\
{\left[\mathrm{mmol}^{*} \mathrm{~g}^{-2 *} \mathrm{~s}^{-1}\right]}\end{array}$ \\
\hline $11: 38$ & $\mathbf{6 3 . 4 1 7} \pm 3.088$ & $\mathbf{0 . 5 8 0} \pm 0.061$ & $11: 58$ & $\mathbf{6 4 . 4 1 1} \pm 3.465$ & $\mathbf{0 . 4 0 4} \pm 0.025$ \\
$13: 08$ & $\mathbf{7 0 . 7 2 2} \pm 2.480$ & $\mathbf{0 . 6 6 4} \pm 0.080$ & $13: 17$ & $\mathbf{6 5 . 0 1 7} \pm 3.178$ & $\mathbf{0 . 4 1 6} \pm 0.029$ \\
$15: 14$ & $\mathbf{6 5 . 0 6 1} \pm 2.275$ & $\mathbf{0 . 6 1 3} \pm 0.073$ & $15: 21$ & $\mathbf{6 0 . 0 8 9} \pm 2.792$ & $\mathbf{0 . 3 8 6} \pm 0.029$ \\
$16: 29$ & $\mathbf{6 3 . 5 0 0} \pm 1.584$ & $\mathbf{0 . 6 0 0} \pm 0.077$ & $16: 40$ & $\mathbf{5 7 . 4 5 6} \pm 3.291$ & $\mathbf{0 . 3 6 9} \pm 0.027$ \\
$17: 51$ & $\mathbf{5 5 . 2 7 8} \pm 2.192$ & $\mathbf{0 . 5 1 2} \pm 0.056$ & $17: 57$ & $\mathbf{5 0 . 5 8 3} \pm 2.877$ & $\mathbf{0 . 3 2 6} \pm 0.026$ \\
$18: 58$ & $\mathbf{4 6 . 7 7 2} \pm 3.036$ & $\mathbf{0 . 4 4 9} \pm 0.063$ & $19: 04$ & $\mathbf{3 9 . 0 1 7} \pm 2.560$ & $\mathbf{0 . 2 5 4} \pm 0.022$ \\
\hline
\end{tabular}


Tab. 29: Kohlenstoffisotopenverhältnisse $\left(\delta^{13} C\right)$ der untersuchten Arten 1999. Flächenmittelwerte von 12 Pflanzen (bei Alhagi) bzw. 6 Pflanzen (andere Arten) mit Standardfehler. $\mathbf{F}$ bewässert (flooded), D unbewässert (dry), $\mathbf{N}$ ungenutzt (not used)

\begin{tabular}{|c|c|c|c|c|}
\hline 1999 & \multicolumn{2}{|c|}{ Alhagi sparsifolia } & \multicolumn{2}{|c|}{ Calligonum caput-medusae } \\
\hline Termin & AFN & ADN & CFN & CDN \\
\hline April & $-24.16 \pm 0.24$ & $-24.49 \pm 0.11$ & $-11.89 \pm 0.12$ & $-11.88 \pm 0.16$ \\
\hline Mai & $-26.13 \pm 0.10$ & $-26.31 \pm 0.07$ & $-13.06 \pm 0.23$ & $-12.92 \pm 0.07$ \\
\hline Juni & $-26.90 \pm 0.17$ & $-27.21 \pm 0.10$ & $-13.41 \pm 0.10$ & $-13.08 \pm 0.10$ \\
\hline Juli & $-27.36 \pm 0.16$ & $-27.43 \pm 0.11$ & $-13.42 \pm 0.25$ & $-13.39 \pm 0.07$ \\
\hline Auqust & $-27.36 \pm 0.16$ & $-27.52 \pm 0.10$ & $-13.90 \pm 0.11$ & $-13.67 \pm 0.12$ \\
\hline September & $-27.26 \pm 0.10$ & $-26.89 \pm 0.13$ & $-13.87 \pm 0.13$ & $-13.72 \pm 0.12$ \\
\hline 1999 & \multicolumn{2}{|c|}{ Tamarix ramosissima } & \multicolumn{2}{|c|}{ Populus euphratica } \\
\hline Termin & TFN & TDN & PFN & PDN \\
\hline April & $-24.14 \pm 0.17$ & $-23.48 \pm 0.41$ & $-25.31 \pm 0.21$ & $-25.00 \pm 0.18$ \\
\hline Mai & $-24.89 \pm 0.31$ & $-23.99 \pm 0.23$ & $-26.47 \pm 0.14$ & $-25.87 \pm 0.19$ \\
\hline Juni & $-25.98 \pm 0.15$ & $-24.49 \pm 0.26$ & $-27.01 \pm 0.21$ & $-26.80 \pm 0.25$ \\
\hline Juli & $-26.23 \pm 0.14$ & $-25.11 \pm 0.28$ & $-27.22 \pm 0.04$ & $-26.98 \pm 0.19$ \\
\hline August & $-25.71 \pm 0.32$ & $-24.73 \pm 0.23$ & $-26.99 \pm 0.26$ & $-26.62 \pm 0.14$ \\
\hline September & $-26.40 \pm 0.16$ & $-25.43 \pm 0.45$ & $-27.59 \pm 0.34$ & $-26.76 \pm 0.11$ \\
\hline
\end{tabular}

Tab. 30: Kohlenstoffisotopenverhältnisse $\left(\delta^{13} \mathrm{C}\right)$ von Alhagi sparsifolia 2000.

Flächenmittelwerte von 12 Pflanzen mit Standardfehler. $\mathbf{F}$ bewässert (flooded), $\mathbf{D}$ unbewässert (dry), $\mathbf{N}$ ungenutzt (not used), $\mathbf{U}$ genutzt (used)

\begin{tabular}{|c|c|c|c|c|}
\hline 2000 & \multicolumn{4}{|c|}{ Alhagi sparsifolia } \\
\hline Termin & & & AFU & ADU \\
\hline April & $-25.17 \pm 0.19$ & $-24.37 \pm 0.13$ & $-24.93 \pm 0.18$ & $-24.67 \pm 0.15$ \\
\hline Mai & $-26.46 \pm 0.17$ & $-26.43 \pm 0.07$ & $-26.42 \pm 0.22$ & $-26.52 \pm 0.10$ \\
\hline Juni & $-27.22 \pm 0.17$ & $-27.10 \pm 0.34$ & $-27.27 \pm 0.24$ & $-27.50 \pm 0.14$ \\
\hline Juli & $-27.79 \pm 0.23$ & $-27.11 \pm 0.25$ & $-28.13 \pm 0.19$ & $-27.38 \pm 0.07$ \\
\hline August & $-27.94 \pm 0.22$ & $-26.40 \pm 0.16$ & $-27.24 \pm 0.11$ & $-27.86 \pm 0.16$ \\
\hline September & $-27.96 \pm 0.17$ & $-27.82 \pm 0.27$ & $-27.89 \pm 0.30$ & $-28.13 \pm 0.11$ \\
\hline
\end{tabular}


